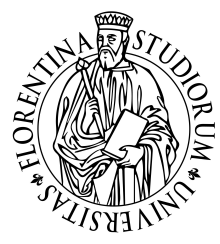

Università degli Studi di Firenze

Scuola di Ingegneria

DIEF - Department of Industrial Engineering of Florence

PhD School: Ingegneria Energetica e Tecnologie Industrali Innovative Scientific Area: ING-IND/08 - Macchine a Fluido

\title{
A 3D COUPLED APPROACH FOR THE THERMAL DESIGN OF AERO-ENGINE COMBUSTOR LINERS
}

PhD Candidate: InG. Lorenzo MazzeI

Tutor: Prof. Ing. Bruno Facchini

Academic Supervisor: Dr. Ing. Antonio Andreini

Industrial Supervisor: ING. FABIO TURRINI

PhD School Coordinator: Prof. Ing. Maurizio De Lucia 

Dedicato a tutti quelli che mi hanno supportato, e sopportato, in questi anni. Grazie di cuore. 



\section{Acknowledgements}

At the conclusion of a journey, it is normal to find yourself thinking about all the people who contribute to the achievement of such an important goal. These few lines are certainly not adequate to express my deep gratitude for you all.

First of all, I would like to thank Prof. Bruno Facchini for this growth opportunity, not only from a professional, but also from a personal point of view.

I would also like to thank Ing. Fabio Turrini for his always valuable suggestions; it is indeed a pleasure and an honour to have the possibility to work alongside GE Avio.

My sincere thanks go also to Ing. Antonio Andreini, for his continuous supervision. My achievements would be unattainable without his help.

My gratitude to the Ergon-guys, Lorenzo, Mirko and, in particular, Ricca (the Master) and Cosimo for the substantial guidance, also beyond this work.

Many thanks and my warmest regards to my former colleagues: Alessio B., Andrea ("si more tutti!"), Maio, Gianlucuccio (thanks for your precious friendship) and Lucuccio (without your assistance this result would not be possible).

A special thank to my colleagues, especially Alessandro (how many trips together!), Carlo, Daniele, Emanuele, Leopoldo, Lorenzo, Marco (we did it, at last!), Sabrina, Stefano and Tommaso for supporting (and standing) me, as well as the guys at THT-Lab: Tommaso, Riccardo and Picchiuccio 
(one of the most beautiful surprises of this year).

Moreover, I would like to thank my whole family for believing in me, Caterina $\&$ Andrea, the best relatives (and friends!) one could wish for, and Annalisa $\&$ Sauro, my second family in the past two years, for giving me everything without expecting anything in return.

At last, I thank Serena, the most important person in my life, the reason for everything and the engine that drives me through the challenges of the future.

This work has been carried out in the framework of several European research projects. The consortia are gratefully acknowledged for the permission to publish the outcomes of these activity and, most of all, for the opportunity to take part in these top-level research initiatives. 
La regola secondo me è: quando sei a un bivio e trovi una strada che va in su e una che va in giù, piglia quella che va in su. È più facile andare in discesa, ma alla fine ti trovi in un buco. A salire c'è più speranza. $\grave{E}$ difficile, è un altro modo di vedere le cose, è una sfida, ti tiene all'erta.

Tiziano Terzani 



\section{Abstract}

The recent limitations imposed by ICAO-CAEP towards a drastic reduction of $\mathrm{NO}_{x}$ emissions is driving the development of modern aeroengines towards the implementation of lean burn concept. The increased amount of air dedicated to the combustion process (up to 70\%) involves several technological issues, including a significant reduction of coolant available for the thermal management of combustor liners. This, from a design perspective, involves the continuous research for effective cooling schemes, such as effusion cooling, and the necessity of more accurate methodologies for the estimation of metal temperature, so as to properly assess the expected duration of hot gas path components.

The flame stabilization through swirler characterized by large effective area leads to extended recirculating zones, which interacts considerably with the liner cooling system. As highlighted in the first part of this dissertation, the impact on the near-wall flow field makes any consideration based on a correlative approach untrustworthy, demanding for more reliable evaluations through CFD analysis. Unfortunately, the application of effusion cooling entails a huge computational effort due to the high number of film cooling holes involved, therefore many approaches have been proposed in literature with the aim of modelling the coolant injection through mass sources.

This work presents SAFE ( Source based effusion model), a methodology for the CFD simulation of the entire combustor, which is based on the local coolant injection through point sources and a calculation of mass flow rate according to local flow conditions. A further step in reduction in 
the computational effort is represented by a different methodology, called Therm3D, which involves the simulation of the flame tube, whereas the solution of the remaining part of the combustor is fulfilled through the modelling of an equivalent flow network, which provides for the estimation of flow split and cold side heat loads.

Ultimately, this work introduce innovative approaches for the CFD investigation of effusion cooled combustor, with a special focus on the metal temperature prediction. A model for the film cooling injection is proposed to overcome the issues related to the necessity of meshing the perforation, nevertheless several improvable aspects have been highlighted, pointing the way for further enhancements. 


\section{Contents}

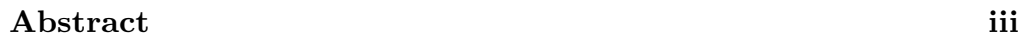

$\begin{array}{ll}\text { Contents ix } & \text { ix }\end{array}$

$\begin{array}{ll}\text { List of Figures } & \text { xvi }\end{array}$

List of Tables $\quad$ xvii

Nomenclature xix

$\begin{array}{ll}\text { Introduction } & 1\end{array}$

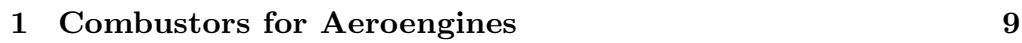

1.1 RQL Combustors . . . . . . . . . . . . . . . . . . . . . . 10

1.2 Lean Combustors . . . . . . . . . . . . . . . . . . 14

2 Heat Transfer in Gas Turbine Combustors 21

2.1 Flow and temperature field in aero-engine combustors . . 22

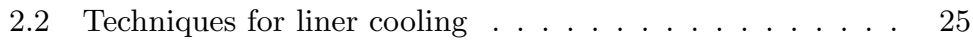

$2.2 .1 \quad$ Effusion Cooling . . . . . . . . . . . . . . . . . . 28

$2.2 .1 .1 \quad$ Geometrical parameters . . . . . . . . . 30

2.2 .1 .2 Operating parameters . . . . . . . . . . 31

$2.2 .1 .3 \quad$ Performance parameters . . . . . . . . . . 32

$2.2 .2 \quad$ Literature Review of Effusion Cooling for Combustor Liners . . . . . . . . . . . . . . . . . . . . 34 
3 CFD Investigation of Combustor Cooling 41

3.1 Numerical study of film cooling . . . . . . . . . . . . . . . 44

$3.2 \quad$ Flat effusion cooling plate . . . . . . . . . . . . . . . . . 46

$3.2 .1 \quad$ Introduction . . . . . . . . . . . . . . . 46

$3.2 .2 \quad$ Investigated test case . . . . . . . . . . . . . . 47

3.2.3 Numerical setup . . . . . . . . . . . . . . . 47

3.2.3.1 Algebraic anisotropic correction . . . . . 49

3.2.3.2 Source Terms . . . . . . . . . . . . . 51

3.2.4 Results .................. . . 51

3.2.4.1 Mesh sensitivity analysis . . . . . . . . 51

3.2.4.2 Turbulence model sensitivity analysis . . 52

3.2.4.3 Blowing ratio effects . . . . . . . . . . 54

3.2 .4 .4 Inlet turbulence effects . . . . . . . . . . 58

3.2.4.5 Density ratio effects . . . . . . . . . . . . 59

3.2.5 Remarks. . . . . . . . . . . . . . . . 60

3.3 Tubular effusion cooled combustor . . . . . . . . . . . . . 63

3.3 .1 Introduction . . . . . . . . . . . . . . 63

3.3 .2 Investigated test case . . . . . . . . . . . 64

3.3.3 Numerical setup . . . . . . . . . . . . . . 66

3.3.3.1 $\quad$ Numerical settings . . . . . . . . . . . 66

3.3.3.2 Boundary conditions . . . . . . . . . . . 66

3.3.3.3 Grid generation . . . . . . . . . . . . 67

3.3.4 Results . . . . . . . . . . . . . . . 67

3.3.4.1 Perforation angle effects . . . . . . . . . . 68

3.3.4.2 Density Ratio effects. . . . . . . . . . . 70

\begin{tabular}{lll}
\hline 3.3 .4 .3 & Comparison with flat plate configuration & 72
\end{tabular}

3.3.4.4 Slot effects . . . . . . . . . . . . 75

3.3.5 Remarks. . . . . . . . . . . . . . . . 78

3.4 Annular slot cooled combustor . . . . . . . . . . . . . . . 80

3.4 .1 Introduction . . . . . . . . . . . . . . . . . . . 80

3.4 .2 Investigated test case . . . . . . . . . . . . . 83

3.4.2.1 PIV measurements. . . . . . . . . . . . . 84

3.4.2.2 HTC measurements . . . . . . . . . . . 85 
3.4 .2 .3 Test conditions . . . . . . . . . . . 86

$3.4 .3 \quad$ Numerical setup . . . . . . . . . . . . . . . . . . . 87

$3.4 .3 .1 \quad$ Boundary conditions. . . . . . . . . . 88

$3.4 .3 .2 \quad$ Grid generation . . . . . . . . . . . . . 89

3.4.3.3 Turbulence modelling - Detached Eddy Simulation . . . . . . . . . . . 89

3.4.3.4 Turbulence modelling - Scale Adaptive Simulation . . . . . . . . . . 91

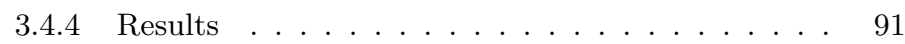

3.4.4.1 Flow field characteristics . . . . . . . . . 91

3.4.4.2 Heat transfer augmentation. . . . . . . . 96

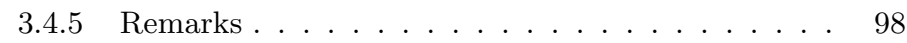

$3.5 \quad$ Concluding remarks $\ldots \ldots \ldots \ldots 1$

4 Development and Validation of a Film Cooling Model 103

4.1 Introduction . . . . . . . . . . . . . . . . . . . 103

4.2 State-of-the-art . . . . . . . . . . . . . . . . . . . . . . 104

4.3 Model development . . . . . . . . . . . . . . . . . . . . . 105

$4.4 \quad$ Model validation $\ldots \ldots \ldots$

$4.4 .1 \quad$ Experimental test case . . . . . . . . . . . . . . 109

4.4 .2 Numerical setup . . . . . . . . . . . . . . . . . 110

4.4 .3 Results: Sensitivity analyses. . . . . . . . . . . . 111

4.4 .3 .1 Detailed simulations . . . . . . . . . . . . 111

4.4 .3 .2 Sensitivity to surface mesh size . . . . . . 112

4.4 .3 .3 Sensitivity to volume element size . . . . 114

4.4 .3 .4 Sensitivity to heat sink value . . . . . . 116

4.4 .3 .5 Sensitivity to heat sink discretization . . 116

$4.4 .3 .6 \quad$ Sensitivity to blowing ratio . . . . . . . . 117

4.4 .4 Results: Pressure drop based formulation . . . . . 118

4.5 Model application: TECC-AE combustor . . . . . . . . . 121

4.5 .1 Description of the test case . . . . . . . . . . . . . 121

4.5 .2 Numerical setup . . . . . . . . . . . . . . . . . . 121

4.5 .3 Results $\ldots \ldots \ldots \ldots \ldots$ 
4.5 .4 Conclusions . . . . . . . . . . . . . . . . . 125

4.6 Model application: NEWAC combustor . . . . . . . . . 127

$4.6 .1 \quad$ Description of the test case . . . . . . . . . . 128

$4.6 .2 \quad$ Numerical setup . . . . . . . . . . . . . . . . . . . 129

4.6 .3 Results $\ldots \ldots \ldots \ldots$

$4.6 .3 .1 \quad$ Flow field and pressure distribution . . . 133

4.6 .3 .2 Temperature field . . . . . . . . . . . 135

4.6 .4 Conclusions . . . . . . . . . . . . . . . . . . . . 142

4.7 Model application: LEMCOTEC . . . . . . . . . . . . 144

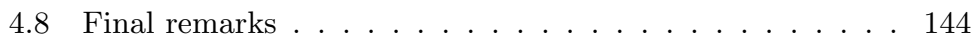

5 Methodologies for thermal design of aero-engine combustors 147

5.1 Preliminary design: Therm1D . . . . . . . . . . . . . 148

$5.1 .1 \quad$ 1D thermal methodologies . . . . . . . . . . . . . 148

5.1 .2 Therm1D . . . . . . . . . . . . . . . . . . . . . . 149

5.1 .2 .1 Gas side radiation . . . . . . . . . . 152

5.1 .2 .2 Coolant side radiation . . . . . . . . . . 154

5.1 .2 .3 Gas side convection . . . . . . . . . . 154

$5.1 .2 .4 \quad$ Coolant side convection . . . . . . . . 155

$5.1 .2 .5 \quad$ Heat $\operatorname{sink} \ldots \ldots \ldots \ldots$. . . . . . . 155

$5.1 .2 .6 \quad$ Film cooling performance . . . . . . . . 155

5.1 .3 Final remarks . . . . . . . . . . . . . . . . . 157

5.2 Detailed design: Therm3D . . . . . . . . . . . . . . . . 158

$5.2 .1 \quad$ Concept $\ldots \ldots \ldots \ldots \ldots$

5.2 .1 .1 Hot side BCs calculation . . . . . . . . 159

5.2 .1 .2 Cold side BCs calculation . . . . . . . . 160

5.2 .1 .3 Thermal calculation . . . . . . . . . . 160

$5.2 .2 \quad$ Development $\ldots \ldots \ldots \ldots 161$

$5.2 .2 .1 \quad$ Mass flow rate calculation. . . . . . . . . 164

5.2 .2 .2 Cold side BCs calculation . . . . . . . . . 164

5.3 Final remarks . . . . . . . . . . . . . . . . . . . . . . 165 
6 Application of Therm3D to NEWAC Combustor 167

6.1 Numerical settings . . . . . . . . . . . . . . 167

6.1 .1 CFD simulation . . . . . . . . . . . 167

6.1 .2 Flow network . . . . . . . . . . . . . 171

6.2 Results . . . . . . . . . . . . . . . 172

6.2 .1 Mesh sensitivity . . . . . . . . . . . . 173

6.2 .2 Radiation modelling sensitivity . . . . . . . . . . 175

6.3 Potential improvements . . . . . . . . . . . . . . . . 175

6.3 .1 Effusion cooling modelling . . . . . . . . . . . . . . 175

6.3 .2 Turbulence modelling . . . . . . . . . . . . . 177

6.3 .3 Radiative heat transfer. . . . . . . . . . . . . . . . 178

6.3 .4 Flow network . . . . . . . . . . . . . . . . . . . 179

6.4 Final remarks . . . . . . . . . . . . . . 179

\begin{tabular}{lr}
\hline Conclusions & 181
\end{tabular}

\begin{tabular}{ll}
\hline Bibliography & 200
\end{tabular} 



\section{List of Figures}

$1 \quad$ Progress of Turbine Inlet Temperature (TIT) over the years

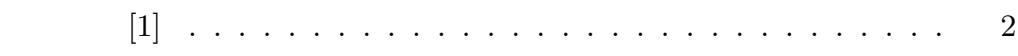

2 Progress of Overall Pressure Ratio (OPR) over the years [1] 2

3 RQL (a) and Lean (b) combustor concepts [5] . . . . . . 4

$1.1 \quad \mathrm{NO}_{x}$ emission limits for engines above FN $20000 \mathrm{lbf}(89$ $\mathrm{kN}$ ) as a function of OPR [9] . . . . . . . . . . 10

1.2 Dependence of emission on Fuel Air Ratio . . . . . . . . . 11

1.3 Rich-Quench-Lean combustor concept . . . . . . . . . . . 12

1.4 Advanced RQL combustor (Pratt \& Whitney PW1500 TALON X) [1] . . . . . . . . . . . . . . . . . . . . . . 13

1.5 Dual Annular Combustors . . . . . . . . . . . . . . . . 15

1.6 TAPS Fuel Injection Concept [14] . . . . . . . . . . . . . . 16

1.7 Low $\mathrm{NO}_{x}$ injectors developed in the context of NEWAC

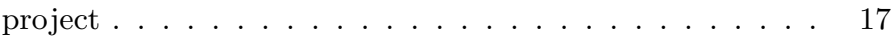

$1.8 \mathrm{NO}_{x}$ emission reduction target of NEWAC EU project [15] 18

$2.1 \quad$ Flow field measurement of a PERM injector in isothermal (left) and reacting/stable condition (right) [26] . . . . . . 24

2.2 Flow field measurement of a LDI injector in isothermal condition [28] $\ldots \ldots \ldots \ldots \ldots \ldots$

2.3 Example of liner cooling arrangement (P\&W JT9D) . . . 27

2.4 Effusion cooled liner . . . . . . . . . . . . . . . . . . . . . . 29 
2.5 Potential coolant related to the application of different cooling techniques . . . . . . . . . . . . . . 30

$2.6 \quad$ Main geometrical parameters for effusion cooling arrangements . . . . . . . . . . . . . . . . 31

2.7 Schlieren flow traces, $2^{\text {nd }}$ and $3^{\text {rd }}$ effusion row observed [40] 35

2.8 Film cooling effectiveness near combustor dilution hole [4] 37

3.1 Sketch of the experimental test rig . . . . . . . . . . . . . 45

3.2 Sketch of the experimental test rig . . . . . . . . . . . . . 48

3.3 Sketch of the numerical domain and particular of the mesh 50

$3.4 \quad$ Sensitivity to mesh refinement $(\mathrm{G} 2, B R=2)$. . . . . . . . 53

3.5 Contour plot of coolant concentration at $X / D=1$ for $9^{\text {th }}$ hole $(\mathrm{G} 2, B R=2)$. . . . . . . . . . . . . . . . . . . 53

3.6 Turbulence model effects on adiabatic effectiveness for both geometries. . . . . . . . . . . . . . . 55

$3.7 \quad B R$ effects on adiabatic effectiveness (G2). . . . . . . . . . 56

$3.8 \quad B R$ effects on adiabatic effectiveness (G7). . . . . . . . . . 57

3.9 Contour plot of coolant concentration on symmetry plane for both geometries (for $1^{s t}, 3^{\text {rd }}$ and $5^{\text {th }}$ rows). . . . . . . 58

3.10 Inlet turbulence effects on adiabatic effectiveness (G2). . . 59

3.11 Comparisons between numerical and experimental data. . 61

3.12 Effcool contour plot on symmetry plane. . . . . . . . . . . 61

3.13 Test case geometries and computational grid. . . . . . . . 65

3.14 Influence of perforation angle $(D R=1)$. . . . . . . . . . 68

3.15 Laterally averaged adiabatic effectiveness profile on liner wall: perforation angle influence $(D R=1)$. . . . . . . . 70

3.16 Density ratio effect on adiabatic effectiveness distribution on liner wall. . . . . . . . . . . . . . . . . . . . . . . . . . 71

3.17 Laterally averaged adiabatic effectiveness profile on liner wall: $D R$ influence. . . . . . . . . . . . . . . . . . . . . . 73

3.18 Local blowing ratio along the liner: influence of DR and perforation. . . . . . . . . . . . . . . . 74 
3.19 Laterally averaged adiabatic effectiveness profile on liner wall: comparison between flat plate and tubular combustor $(D R=1, B R=2)$. . . . . . . . . . . . . . . . 74

3.20 Slot coolant effect on adiabatic effectiveness distribution on liner wall. . . . . . . . . . . . . . . . . . . . . . . . . . 76

3.21 Slot coolant distribution on meridional plane. . . . . . . . 77

3.22 Laterally averaged adiabatic effectiveness profile on liner wall: slot cooling injection influence. . . . . . . . . . . . 77

3.23 Laterally averaged adiabatic effectiveness profile on liner wall: slot cooling mass flow rate influence. . . . . . . . . . 79

3.24 Measured and predicted flow field on the median plane . . 81

3.25 Classical flow pattern of an array of swirles (top) and dome-attached swirling flow (bottom) [92] . . . . . . . . . 82

3.26 Experimental apparatus . . . . . . . . . . . . . . 83

3.27 Position of the PIV measurements planes . . . . . . . . . 85

3.28 Sketch of the computational domain . . . . . . . . . . . . 88

3.29 Computational grids: Mesh 1 (left) and 2 (right) . . . . . 89

3.30 Flow field on the center plane . . . . . . . . . . . . . . . . 93

3.31 Profiles of velocity component in stream-wise direction (center plane) . . . . . . . . . . . . . . . . 94

3.32 Effect of mesh refinement and turbulence modelling on $M$ criterion (center plane) . . . . . . . . . . . . . . . . . . . 96

3.33 2D velocity on the median plane: PVC visualized by a constant pressure isosurface and coloured by stream-wise velocity . . . . . . . . . . . . . . . . . 97

3.34 Nusselt number distributions on the central sector . . . . 99

3.35 Sketch of the main recirculation structures inside the test section . . . . . . . . . . . . . . . . . . . . 100

3.36 Laterally averaged Nusselt number augmentation . . . . . 100

4.1 Conceptual representation of effusion hole modelling . . . 106

4.2 Sketch of mass flow rate calculation . . . . . . . . . . . . 107

4.3 Sketch of the computational domain for detailed simulations 11 
4.4 Results for detailed simulations $(\mathrm{M}=5)$. . . . . . . . . . . 112

4.5 Effect of Max Size $(G 2, M=5)$. . . . . . . . . . . . . . . 113

4.6 Sketch of source point application in CFX . . . . . . . . . 114

4.7 Effect of the parameter Tetra Size Ratio on the volume element size . . . . . . . . . . . . . . . . . . . . . 115

4.8 Effect of Tetra Size Ratio (G2, M=5) . . . . . . . . . . . . 115

4.9 Effect of heat sink value $(\mathrm{G} 2, \mathrm{M}=5)$. . . . . . . . . . . . 116

4.10 Heat removal discretisation on one (left) and three (right) heat sinks . . . . . . . . . . . . . . . 117

4.11 Effect of blowing ratio (G2, $\mathrm{M}=5,3,1.5)$. . . . . . . . . . 118

4.12 Comparison of the flowfield in presence of discrete holes (up) and source points (down) $\left(\mathrm{G} 2,1^{\text {st }}\right.$ row, M=5) . . . . 118

4.13 Final comparison between experimental data, source model \begin{tabular}{|l|}
\hline with prescribed values and pressure drop formulation: effect \\
\hline of geometry $(\mathrm{M}=5)$. . . . . . . . . . . . . . . . 120
\end{tabular}

4.14 Final comparison between experimental data, source model with prescribed values and pressure drop formulation: effect of blowing ratio (G2) . . . . . . . . . . . . . . . 120

4.15 Flow field in the combustor meridional plane (without radiation) . . . . . . . . . . . . . . . 122

4.16 Flow field in the combustor meridional plane: detail of the primary zone with velocity vectors (without radiation)] . . 123

4.17 Stream-wise distribution of predicted discharge coefficients 124

4.18 Predicted flow split within the combustor . . . . . . . . . 124

4.19 Temperature field of the combustor (without radiation) . 125

4.20 Temperature contour on liner and heat shield (without radiation) . . . . . . . . . . . . . . . . 125

4.21 Temperature profiles along heat shield and liner. . . . . . 126

4.22 GE Avio's NEWAC combustor prototype . . . . . . . . . 128

4.23 Sketch of the injection system . . . . . . . . . . . . . . . . 129

4.24 Sketch of the computational domain and assigned boundary conditions . . . . . . . . . . . . . . . . . . . 132

4.25 Computational mesh of the fluid domain (Mesh C) . . . . 133 
4.26 Flow field within the combustor . . . . . . . . . . . . . . 134

4.27 Comparison between experimental and predicted pressure losses. . . . . . . . . . . . . . . . 135

4.28 Temperature field within the combustor . . . . . . . . . . 136

4.29 Contour of metal temperature . . . . . . . . . . . . . 137

4.30 Comparison between experimental and predicted values of metal temperature . . . . . . . . . . . . . . 138

4.31 Comparison between experimental and predicted values of metal temperature . . . . . . . . . . . . . . . 139

4.32 Contour of transported passive scalar Coolant . . . . . . . 141

4.33 Coolant field within the combustor . . . . . . . . . . . . . 141

4.34 Coolant distributions at increasing distance from the injection systems . . . . . . . . . . . . . . . . . . . 142

4.35 Configurations investigated in the framework of LEMCOTEC project. . . . . . . . . . . . . . . . 144

5.1 Conceptual illustration of 1D thermal analysis. . . . . . . 150

5.2 Therm1D: procedure flow chart . . . . . . . . . . . . 152

5.3 Sketch of CFD domain . . . . . . . . . . . . . . . . . . . . 160

5.4 Sketch of flow network . . . . . . . . . . . . . . . . . . . . 161

5.5 Sketch of boundary conditions applied to solid domain . . 162

$5.6 \quad$ Sketch of codes coupling . . . . . . . . . . . . . . . . . 162

5.7 Coupling between CFD and Flow Network (FN) . . . . . 163

5.8 Implementation of User Fortran code in CFX . . . . . . . 163

6.1 Sketch of the computational domain . . . . . . . . . . . 169

6.2 computational grids for mesh sensitivity analysis . . . . . 170

6.3 Sketch of equivalent combustor flow network. . . . . . . . 171

6.4 Position of thermocouples . . . . . . . . . . . . . 173

6.5 Mesh sensitivity analysis: metal temperature distribution on cold side . . . . . . . . . . . . . . . . . . . . . 174

6.6 Radiation modelling sensitivity analysis: metal temperature distribution on cold side . . . . . . . . . . . . . . . . 176 
6.7 Distributed mass source model: mesh elements for source application . . . . . . . . . . . . . . . . . . 176

6.8 Comparison between effusion cooling models: span-wise

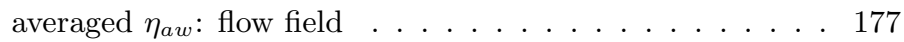

6.9 Comparison between effusion cooling models: span-wise averaged $\eta_{a w}$ : adiabatic effectiveness . . . . . . . . . . . . 178 


\section{List of Tables}

3.1 Test plate geometry . . . . . . . . . . . . . . . . . . . 47

3.2 Test matrix of performed simulations. . . . . . . . . . . . . 48

3.3 Test matrix. . . . . . . . . . . . . . . . . . . . . . . . . 65

3.4 Boundary conditions for additional transport equations. . 67

3.5 Main features of the computational grid . . . . . . . . . . 89

4.1 Test case outline . . . . . . . . . . . . . . . . . . . . . . . 109

4.2 Grids used in the assessment of the sensitivity to $2 \mathrm{D}$ element dimension . . . . . . . . . . . . . . 113

4.3 Outline of $M S / D_{h}$ values for each grid used . . . . . . . . 113

4.4 Comparison between experimental and predicted mass flow rate varying the blowing ratio $(G 2, M=1.5,3,5))$. . . . 121

4.5 Description of the investigated test point. . . . . . . . . . 129

4.6 Grids generated for the fluid domain . . . . . . . . . . . . 133

5.1 Operating parameters for the implemented correlations. . 156

6.1 Main features of the computational grid . . . . . . . . . . 170 



\section{Nomenclature}

$\begin{array}{lll}A & \text { Area } & {\left[\mathrm{m}^{2}\right]} \\ B R & \text { Blowing Ratio } & {[-]} \\ C_{D} & \text { Discharge coefficient } & {[-]} \\ c_{p} & \text { Spec. heat capacity at const. P } & {\left[\mathrm{J} \mathrm{kg}^{-1} \mathrm{~K}^{-1}\right]} \\ d / D & \text { Diameter } & {[\mathrm{m}]} \\ D R & \text { Density Ratio } & {[-]} \\ f & \text { Friction factor } & {[-]} \\ F A R & \text { Fuel to Air Ratio } & {[-]} \\ h / H T C & \text { Heat Transfer Coefficient } & {\left[\mathrm{W} \mathrm{m} \mathrm{m}^{-2} \mathrm{~K}^{-1}\right]} \\ H & \text { Height } & {[\mathrm{m}]} \\ I & \text { Momentum Flux Ratio } & {[-]} \\ k & \text { Turbulence kinetic energy } & {\left[\mathrm{m}^{2} \mathrm{~s}^{-2}\right]} \\ k & \text { Heat conductivity } & {\left[\mathrm{W} \mathrm{m} \mathrm{K}^{-1} \mathrm{~K}^{-1}\right]} \\ L & \text { Length } & {[\mathrm{m}]} \\ l_{b} & \text { Beam length } & {[\mathrm{m}]} \\ L e & \text { Lewis number } & {[-]} \\ L F & \text { Luminosity Factor } & {[-]} \\ m & \text { Mass flow rate } & {[\mathrm{kg} \mathrm{s}]} \\ M & \text { Blowing Ratio } & {[-]} \\ M & \text { Pope criterion } & {[-]} \\ M S & \text { Mesh Size } & {[\mathrm{mm}]} \\ N H F R & \text { Net Heat Flux Reduction } & {[-]} \\ N u & \text { Nusselt number } & {[-]} \\ O P R & \text { Overall Pressure Ratio } & {[-]} \\ P & \text { Pressure } & {[\mathrm{Pa}]} \\ q r & \text { Prandtl number } & {[-]} \\ R & \text { Wall heat flux } & {\left[\mathrm{W} \mathrm{kg}^{-1} \mathrm{~K}^{-1}\right]} \\ & \text { Gas constant } & \end{array}$




$\begin{array}{lll}R e & \text { Reynolds number } & {[-]} \\ S & \text { Pitch } & {[\mathrm{m}]} \\ S_{N} & \text { Swirl number } & {[-]} \\ S c & \text { Schmidt number } & {[-]} \\ T & \text { Temperature } & {[\mathrm{K}]} \\ T I T & \text { Turbine Inlet Temperature } & {[\mathrm{K}]} \\ T u & \text { turbulence intensity } & {[-]} \\ v / V & \text { Velocity } & {\left[\mathrm{m} \mathrm{s}^{-1}\right]} \\ V_{h r} & \text { Velocity head ratio } & {[-]} \\ V R & \text { Velocity Ratio } & {[-]} \\ y^{+} & \text {Non-dimensional wall distance } & {[-]}\end{array}$

\section{Acronyms}

ACARE

Adim

$B C$

$C A E P$

$C F D$

CHT

$C V P$

$D A C$

$D E S$

$D N S$

EU

FEM

$F N$

$G G I$

GIS

$H T$

ICAO

IRZ

JCF

$L D I$

$L D A$

$L D V$

$L E S$

$L P P$
Advisory Council for Aeronautics Research in Europe

Non-dimensional

Boundary condition

Committee on Aviation Environmental

Protection

Computational Fluid Dynamics

Conjugate Heat Transfer

Counter-rotating vortex

Dual Annular Combustor

Detached Eddy Simulation

Direct Numerical Simulation

European Union

Finite Element Model

Flow Network

General Grid Interface

Grid Induced Separation

High Turbulence

International Civil Aviation Organization

Inner recirculation zone

Jet in Cross-Flow

Lean Direct Injection

Laser Doppler Anemometry

Laser Doppler Velocimetry

Large Eddy Simulation

Lean Premixed Prevaporized 


$\begin{array}{ll}L T & \text { Low Turbulence } \\ O R Z & \text { Outer recirculation zone } \\ P E R M & \text { Partial Evaporation Rapid Mixing } \\ P I V & \text { Particle Image Velocimetry } \\ P O D & \text { Proper Orthogonal Decomposition } \\ P S P & \text { Pressure Sensitive Paint } \\ P V C & \text { Precessing Vortex Core } \\ R A N S & \text { Reynolds Averaged Navier-Stokes } \\ R C Z & \text { Recirculation zone } \\ R Q L & \text { Rich-Quench-Lean } \\ S A C & \text { Single Annular Combustor } \\ S A F E & \text { Source based effusion model } \\ S A S & \text { Scale Adaptive Simulation } \\ S S T & \text { Shear Stress Transport } \\ T A P S & \text { Twin Annular Premix System } \\ T L C & \text { Thermochromic Liquid Crystal } \\ U L N & \text { Ultra Low NOx } \\ U R A N S & \text { Unsteady RANS }\end{array}$

\section{Greeks}

$\alpha$

$\beta$

$\beta$

$\gamma$

$\gamma$

$\epsilon$

$\varepsilon$

$\eta$

$\theta$

$\rho$

$\sigma$

$\sigma$

$\mu$

$\omega$

$\Delta$

$\Phi$

\section{Subscripts}

0

ad
Inclination angle

Pressure ratio

Swirl velocity angle

Compound angle

Heat capacity ratio

Emissivity

Turbulence eddy dissipation

Effectiveness

Inverse of $\eta_{o v}$

Density

Boltzmann constant

Surface porosity

Dynamic viscosity

Turbulence eddy frequency

Spacing

Equivalence Ratio $\left.{ }^{\circ}\right]$

[-]

[0]

[0]

[-]

[-]

$\left[m^{2} s^{-3}\right]$

[-]

[-]

[kg m-3]

[-]

[-]

$[\mathrm{Pa} s]$

$\left[s^{-1}\right]$

$[m]$

[-]
Reference, total

Adiabatic 


$\begin{array}{ll}\text { an } & \text { Annulus } \\ \text { aw } & \text { Adiabatic wall } \\ \text { axi } & \text { Axial direction } \\ c / \text { cool } & \text { Coolant } \\ \text { conv } & \text { Convection } \\ g & \text { Gas } \\ h & \text { Hole } \\ h s & \text { Heat Sink } \\ \text { is } & \text { Isentropic } \\ \text { main } & \text { Mainstream } \\ \text { ov } & \text { Overall } \\ r a d & \text { radiation, radial direction } \\ r e f & \text { Reference } \\ s & \text { Soot } \\ \text { st } & \text { Stoichiometric } \\ T, t & \text { Total } \\ t & \text { Turbulent } \\ \text { tan } & \text { Tangential direction } \\ w & \text { Wall }\end{array}$

\section{Superscripts}

*

$e q$
Reference, non-dimensional

Equivalent 


\title{
Introduction
}

\begin{abstract}
"The airplane, from its inception, has grown in capacity and performance only at the pace permitted by the aircraft engine, and we have historically selected the engine first and designed the airplane around it. The search for high power has never relaxed for an instant." Glenn Martin
\end{abstract}

\section{Developments in aero-engines}

One of the inventions that most contributed to the development of the aviation field is certainly represented by the jet engine. Since its conception, occurred almost concurrently in 1939 thanks to the contributions of von Ohain in Germany and Whittle in England, the technology improvements have been really impressive: according to Ballal and Zelina [1], up to 2003, engine trust has increased over 100-fold and the thrust/weight ratio to 7 . Modern aero-engines can be employed up to 10000 hours before requiring maintenance, whereas the early prototypes barely lasted for less than 10 hours.

Engine thermal efficiency is approaching 50\%, mainly through the progressive increase in Turbine Inlet Temperature (TIT) and Overall Pressure Ratio (OPR), as highlighted in Figg. 1 and 2 Making reference to the first picture, it is evident the TIT has far exceeded the melting point of hot gas path components. Notwithstanding the advances in material properties and the continuous improvements in manufacturing technologies, a more and more essential contribution is ascribable to the 
development of innovative cooling techniques. In addition, the context is made even more difficult by the above mentioned trend characterized by increasing OPR. In fact, its increase involves a higher temperature at the compressor discharge that limits the cooling capacity of the air. For these reasons, the definition of the most appropriate cooling scheme represents one of the most challenging tasks in the combustor and turbine design, since it directly determine the components life.

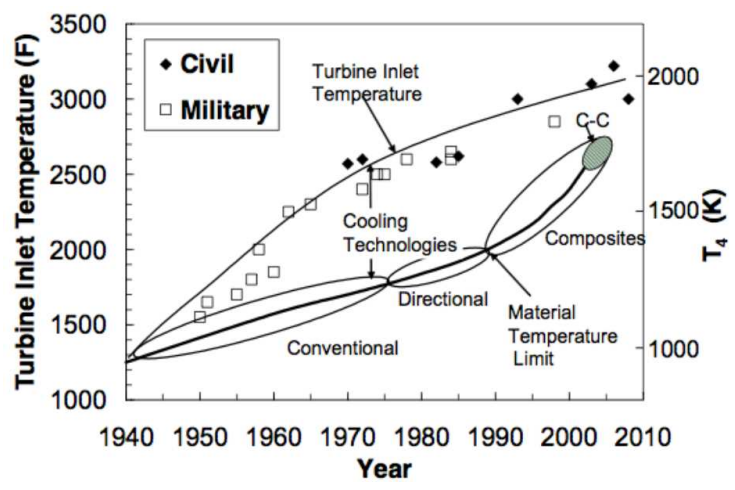

Figure 1: Progress of Turbine Inlet Temperature (TIT) over the years [1]

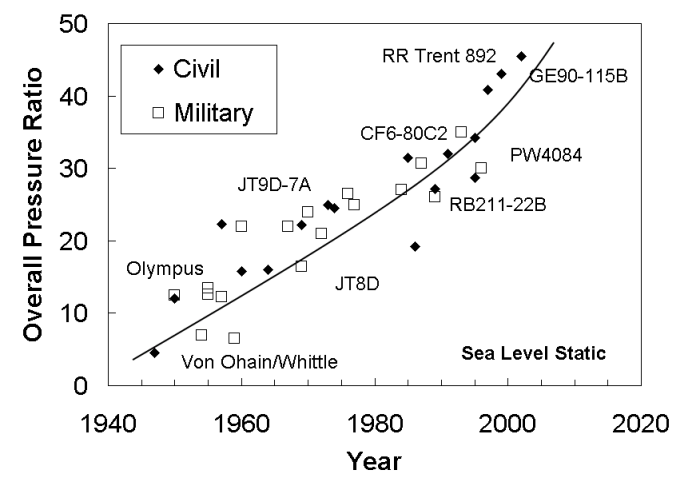

Figure 2: Progress of Overall Pressure Ratio (OPR) over the years [1] 
Nevertheless, many concerns in the design of civil aero-engines are nowadays aimed at meeting the strict regulations for what concerns emissions. Civil air traffic is expected to grow of $5 \%$ (total passenger kilometres) in the period from 2006 to 2026 2], leading to an increase of the quantity of pollutant emissions caused by civil aviation. In order to partially compensate to this increase, the Advisory Council for Aeronautics Research in Europe (ACARE) has set several ambitious goals to be achieved by 2020 (from a 2000 baseline) [3]:

- $50 \%$ reduction in $\mathrm{CO}_{2}$ per passenger kilometre;

- $80 \%$ reduction in $\mathrm{NO}_{x}$ emissions;

- $50 \%$ reduction in noise.

Even though $20 \%$ and $10 \%$ of the $\mathrm{CO}_{2}$ reduction is expected to be achieved through improvements in the airframe and air traffic management and operations, these targets strongly affect the aero-engine. Its design is therefore becoming a matter of optimisation of the whole system, requiring the accurate assessment of trade-offs with the aim of meeting all the requirements. It is worth mentioning, for example, the improvement of the overall efficiency through the increase in TIT and OPR, that results in larger $\mathrm{NO}_{x}$ production.

Many aero-engine manufactures have identified the lean burn combustion technology as the most promising way to reach the required drastic $\mathrm{NO}_{x}$ reduction. Among the many design aspects to be considered in the implementation of lean combustors, a key-point is certainly the design of the liner cooling system, which becomes more critical due to the decreased amount of air available. In fact, compared to classic Rich-Quench-Lean (RQL) combustors (see Fig. 3), up to $70 \%$ of the overall air mass flow is utilized for fuel preparation and control of lean combustion, therefore the goal in future combustor development is to reduce the amount of coolant by about $50 \%$. 4 .

In order to maintain adequate component life target with such a big coolant saving, it is not sufficient to employ the most effective cooling 


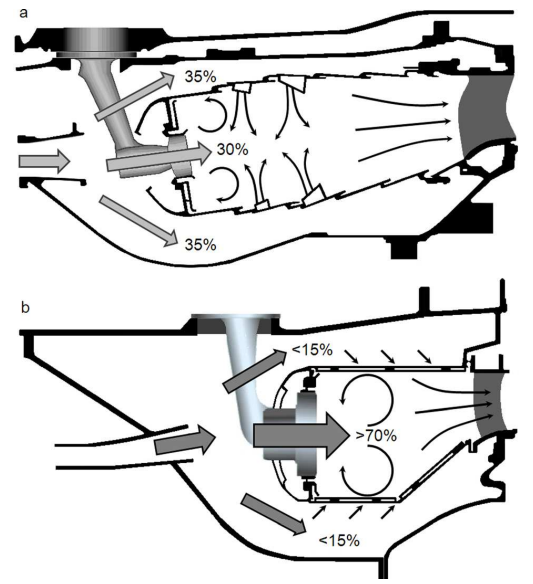

Figure 3: $R Q L$ (a) and Lean (b) combustor concepts [5]

schemes, such as effusion cooling, but it is also required to optimize their design on the actual flow and thermal working regime.

Lean combustion burners are characterized by an internal swirling flow, generating the typical vortex breakdown, which strongly affects the near-wall flow in the proximity of both liners and dome. Thus a reliable estimate of liner thermal protection achieved by means of effusion cooling cannot leave out of consideration the presence of a complex 3D flow field interacting with the coolant jets [6].

In the past, the thermal design of the combustor has been conducted through an empirical approach based on lessons learnt and eased by the large amount of coolant available. The approach proposed by Lefebvre [7], based on the assumption of 1D heat conduction across the liner and the widespread use of correlations for the estimation of convective and radiative thermal loads, could have been considered to be sufficiently accurate due to the one-dimensional characteristics of the flow field, on both cold and hot sides of the liners, mainly ascribable to the significant axial extension of old generation, tubular combustors.

The more compact design and the three-dimensional nature of the 
flame, besides the presence of local coolant injection through discrete holes and their interaction with the swirling flow, have made the assessment of the thermal loads a critical issue. Moreover, the increased competition of the market impose to abandon the trial \& error approach for the benefit of a general reduction of money and time spent for the product development. Within this scenario it looks quite evident that, in a perspective of an accurate metal temperature prediction, the approach for the thermal design of lean combustors requires a deep revision.

\section{Aim of the work}

The main objective of this research is the development of a numerical procedure for the aero-thermal analysis of lean combustors (Therm3D). With a perspective of an application in the industrial context, particular attention has been paid on achieving a good compromise between computational cost and accurate representation of the physical phenomena involved. Keeping in mind these considerations, the methodology has been developed combining these features:

- CFD RANS approach for the simulation of the flow field within the flame tube, aimed at an accurate prediction of hot gas side thermal loads;

- Inclusion of the metal components (liners and dome) within the CFD domain, so as to take into account the 3-D temperature distribution;

- Flow network based solution of the flow field on the coolant side, through a rapid but reliable correlative calculation of the cold side thermal loads.

Furthermore, a great effort has been focused on the development of a model for film cooling injection, since its presence represents a serious issue in the CFD investigation of effusion cooled combustors. The numerical analysis of jets in cross-flow is characterized by well-known problems, due to both modelling issues and the high number of mesh elements 
required for an appropriate spatial discretisation (at least 100000 per hole). Therefore, despite the increasing computational resources, the CFD simulation of an entire combustor still represents a challenge hardly compatible with stringent time requirements typical of industry.

To the purpose of improving the feasibility of this kind of investigations, an innovative approach has been proposed for modelling film cooling injection, so as to avoid the meshing of discrete holes, delivering a significant added value to the CFD simulation of the combustor.

The activity that led to the results presented in this dissertation was carried out in the framework of several EU projects: the development of the effusion cooling model was mainly performed in 2012-2013 during the TECC-AE project, whereas the work on Therm3D took place in 2014 in the context of the IMPACT-AE project, which is devoted to the creation of methodologies for the design of clean combustors. Nevertheless, their validation and application were made possible thanks also to the involvement of the Heat Transfer \& Combustion group within the framwork of other projects such as KIAI, NEWAC and LEMCOTEC.

\section{Thesis Outline}

During this research activity the aspects related to heat transfer in gas turbine combustors have been analysed through both CFD-based and correlative approaches. The main achievements are certainly represented by the effusion cooling model and its implementation within an innovative thermal design procedure. However, since many efforts have been spent in order to deepen the knowledge of effusion cooling and its interaction with the swirling flow, a comprehensive description of lean combustors and the challenges related to the heat transfer in such devices is provided. The dissertation will be organized as it follows:

\section{Chapter 1: Combustors for Aeroengines}

In order to better explain the context into which the work has been carried out, low emission engines are presented. The chapter is focused on the limitations that characterize standard RQL combustors and that moti- 
vate the efforts aimed at the implementation of the lean burn technology, with the issues that entails.

\section{Chapter 2; Heat Transfer in Gas Turbine Combustors}

This part is devoted to clarify the phenomena that characterize the heat transfer in lean combustors. The cooling technologies used to manage the thermal loads have been described, with a particular focus on effusion cooling, and the approaches for their assessment.

\section{Chapter 3; CFD Investigation of Combustor Cooling}

Several activities were carried out with the aim of better understanding the performance of effusion cooling. This chapter allows a deeper comprehension concerning the accuracy in their estimation through both CFD and correlative tools.

\section{Chapter 4: Development and Validation of a Film Cooling Model}

The development of an innovative film cooling model is the main subject of this chapter. After a detailed overview for what concerns the state-of-the-art of this topic, the description of the proposed methodology is provided, giving appropriate emphasis to validation and application on representative test cases.

\section{Chapter 5 Methodologies for thermal design of aero-engine combustors}

This chapter describes the strategies for the thermal design of combustor cooling systems. Therm1D, the in-house code currently used by University of Florence and GE Avio for the preliminary design, is presented. The main objective is to point out the assumptions that make the $1 \mathrm{D}$ approach inadequate for the thermal analysis in the context of a detailed design of lean combustors. In addition, the phases of conception and development of a new thermal design procedure (Therm3D) are described, giving more insight into the most appropriate modelling strategies aimed at achieving the required compromise between computational effort and accuracy.

\section{Chapter 6: Application of Therm3D to NEWAC Combustor}

The application of Therm3D to the aero-thermal investigation of 
a lean burn combustor is presented. The predicted metal temperature distribution have been compared against experimental data and the results obtained using Therm1D.

In the last chapter, a summary of the main achievements of this research is given together with conclusions and recommendations for future research. 


\section{Chapter 1}

\section{Combustors for Aeroengines}

The development of combustors for the next generation of civil aeroengines is driven by the the demand for low emission requirements, in particular $\mathrm{NO}_{x}$. The standards set by ICAO-CAEP (see Fig. 1.1) and the objectives highlighted by ACARE 8 . further complicate the design of the aeroengine combustors, which must satisfy several technical requirements. As mentioned above, the improvement of the thermal efficiency (i.e. of fuel consumption and thus of $\mathrm{CO}_{2}$ ) is achievable through the increase in Overall Pressure Ratio and Turbine Inlet Temperature.

The trend of aeroengine design is therefore moving towards the development of Ultra High Pressure Ratio gas turbines, with OPR greater than 70 [10. Nevertheless, the high pressure level within the combustion chamber, in conjunction with the increase in TIT, promotes the $\mathrm{NO}_{x}$ production, making the reduction of emissions even more challenging, requiring the definition of trade-offs aiming at defining the optimal design of not only the aeroengine, but the whole vehicle.

Amongst the mechanisms that contribute to the nitrous oxides formation, the most relevant for typical aeroengine combustors is that one formulated by Zeldovich, which relates the $\mathrm{NO}_{x}$ production with an exponential dependence on temperature [7. Within the combustion chambers the peak of temperature is achieved in the proximity of the stoichiometric 


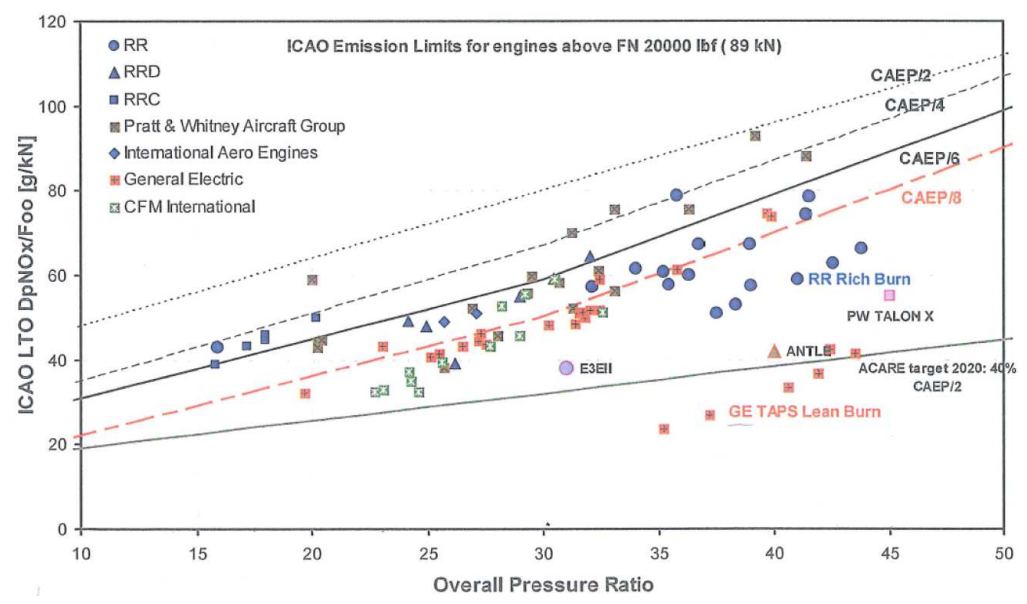

Figure 1.1: $\mathrm{NO}_{x}$ emission limits for engines above FN $20000 \mathrm{lbf}$ (89 kN) as a function of $O P R$ [9]

regions. For this reason, the abatement of $\mathrm{NO}_{x}$ emission involves significant efforts directed at controlling the air-fuel mixture in order to avoid the stoichiometric conditions.

As depicted in Fig. 1.2 two possible operations zone can be exploited with the aim of reducing the nitrous oxides formation: the rich burn and the lean burn mode. In this chapter the most spread implementations of these strategies for low emission combustors are presented: the RQL and the Lean concepts.

\subsection{RQL Combustors}

Most of the aeroengines currently employed in the civil aviation sector are based on the Rich burn - quick Quench - Lean burn (RQL) concept. This technology was proposed in the 1980s in order to achieve a significant reduction in $\mathrm{NO}_{x}$ emission. The schematic mode of operation is depicted in Fig. 1.3a keeping in mind the necessity to avoid flame blow out during the entire flight mission and with any kind of weather conditions, the basic 


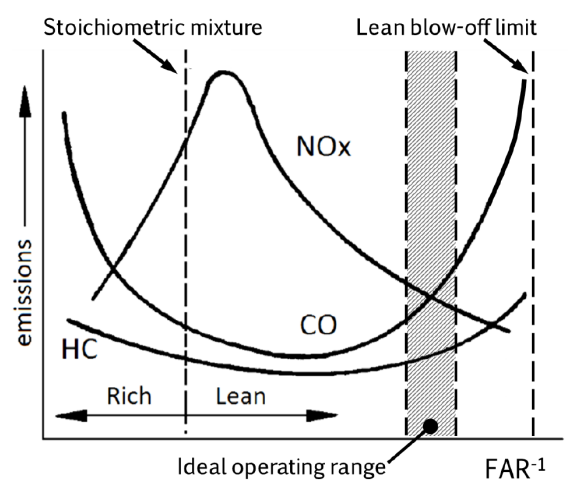

Figure 1.2: Dependence of emission on Fuel Air Ratio

idea consists in providing the flame stability through the combustion of a rich mixture in the primary zone. Moreover, the "rich-burn" condition $(\Phi=1.2-1.6)$ reduces the nitrogen oxides production due to the relatively low temperature and the limited concentration of oxygen containing intermediate species. Subsequently, the gas is diluted through the addition of primary air with the aim of providing a quenching of the reactions and shifting as quick as possible towards the "lean-burn" condition $(\Phi=$ $0.5-0.7)$. It appears therefore evident that the main focus and the technological issues lie in ensuring rapid mixing to minimize the residence time at the stoichiometric condition, at which is associated the maximum $\mathrm{NO}_{x}$ production.

From a more practical point of view, the implementation of the RQL concept in a modern aero-engine is presented in Fig. 1.3b The flame in the primary zone is usually stabilized by means of swirlers, devices able to provide a tangential velocity component to the air with the purpose of generating the swirler breakdown phenomenon and the recirculation of hot gas toward the injector. The secondary zone, immediately downstream of the mixing ports, completes the reactions of the unburnt species $(\mathrm{CO}$, $\mathrm{uHC}$, smoke), whereas additional air is usually injected (not shown in the picture) to control the exit temperature profile. 


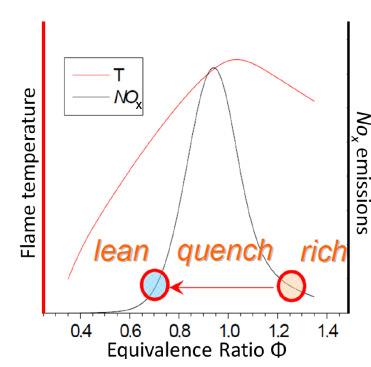

(a) $R Q L$ concept

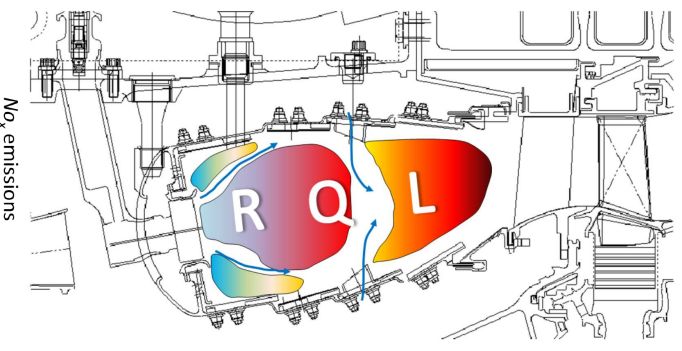

(b) $R Q L$ combustor scheme ( $R R$ Trent $X W B)$

Figure 1.3: Rich-Quench-Lean combustor concept

The intrinsic characteristics of the operation of RQL combustors represents a severe limitation in optimizing the polluting emissions. The critical switching from rich- to lean-burn conditions involves necessarily local values of $\Phi \approx 1$. In addition, the application of film cooling in the primary zone may produce locally stoichiometric conditions, undermining the efforts in reaching the low $\mathrm{NO}_{x}$ target.

Nevertheless, the nitrous oxides do not represent the only type of pollution to be concerned about: the rich condition achieved in the primary zone, as well as the quenching in the proximity of the liners, entails also a significant production of unburnt species, such as $\mathrm{CO}, \mathrm{uHC}$ and soot. The presence of the lean secondary zone is supposed to significantly reduce their emission by reason of the high oxydizer concentration, provided that the temperature reduction due to the mixing is high enough to burn these species. For this reason, the equivalence ratio for both zones must be carefully selected to satisfy all emissions requirements.

In the light of the intrinsic limits of RQL combustors, it is possible to understand the motivations that are pushing towards the implementation of the lean burn concept. Nevertheless, despite the efforts carried out in the last decade by the main aero-engine manufacturers in developing lean combustors, at the moment only General Electric produces aero-engines equipped with this kind of technology. Therefore, RQL combustors still undergo significant attention aimed at their optimization. 
Recent advances have shown that significant reductions in residence time and $\mathrm{NO}_{x}$ production can be achieved without compromising combustor stability and low-power performance. Use of fuel injectors capable of producing small droplets uniformly dispersed, the rapid air jet mixing, as well as the decrease in combustion volume, have demonstrated $\mathrm{NO}_{x}$ reduction of over $50 \%$ when compared to early annular combustors [11. The compact design is advantageous also with regard to the thermal management of the liners, since it mitigates the issues related to the occurrence of stoichiometric conditions in the proximity of film cooled walls.

An example of advanced RQL combustor can be given by the TALON family (Technology for Advanced Low $\mathrm{NO}_{x}$ ), developed by Pratt \& Whitney. These combustors employ the proven and robust technology of RQL, adding drastic improvements focused on obtaining a uniform rich primary zone, optimized quench, advanced cooling and reduced residence time. The last member of the family, the TALON X (see Fig. 1.4, indicated the potential to achieve $\mathrm{NO}_{x}$ levels up to $70 \%$ below CAEP2 regulations [12] and it will be equipped on the PW1000G geared turbo-fan.

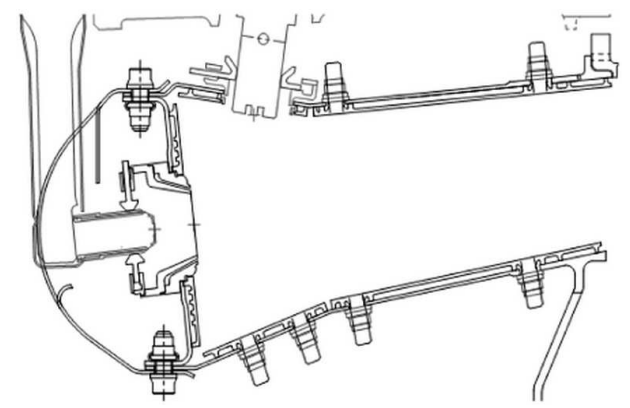

Figure 1.4: Advanced RQL combustor (Pratt E Whitney PW1500 $T A L O N X)$ 11] 


\subsection{Lean Combustors}

Fully premixed combustors have been under development for nearly forty years, yet no airplane with premixed combustion entered into service. The industrial gas turbines have made a successful transition to this technology in a relatively short term, accomplishing substantial reduction in $\mathrm{NO}_{x}$ emissions. The gap must be ascribed to the peculiarities of aeroengines, with the issues related to the preparation of liquid fuel by full or partial prevaporization and premixing and the impact of part load during the flight mission.

The combustor emissions need therefore to be put into the perspective of the other design criteria to be fulfilled. The following list of prioritized combustor design considerations is taken from NASA [13:

- Safety

- Operability

- Efficiency

- Durability

- Emissions

- Downstream turbomachinery thermal and life integrity

Despite the serious concerns and the efforts made to improve emissions, it appears evident that emissions are normally the fourth or fifth combustor design priority and the results achieved can be undermined by design trade-off aimed at improving combustor operability and durability, hence explaining the still challenging application in the aeronautical context.

The first implementation of the lean burn concept occurred through the exploitation of fuel staging. This approach allows to partially overcome the issues related to the variable operating conditions during the mission cycles. It is accomplished dividing the so-called staged combustor in two separated zones, each one optimized to achieve different requirements: the first zone is designed to provide a "pilot" flame operating with a slightly lean, near to stoichiometric, equivalence ratio, hence ensuring 
combustion stability and reduced $\mathrm{CO}$ and $\mathrm{uHC}$ emissions at idle and low power conditions. In addition, at higher power conditions it supplies a heat source for the secondary "main" combustion region, operating with a premixed lean mixture. The burners can be turned-off both individually and in groups, achieving the best performance at different operating conditions, ensuring efficient and stable combustion and minimizing $\mathrm{NO}_{x}$ and smoke emissions.

Examples af axially-staged Dual Annular Combustors (DAC) can be represented by the prototype developed in the context of the CLEAN (Component Validator for Environmentally Friendly Aero Engine) EU project. The DAC family of the GE CFM56 (-5B and $-7 \mathrm{~B})$ is the only product in service that implements this kind of technology.

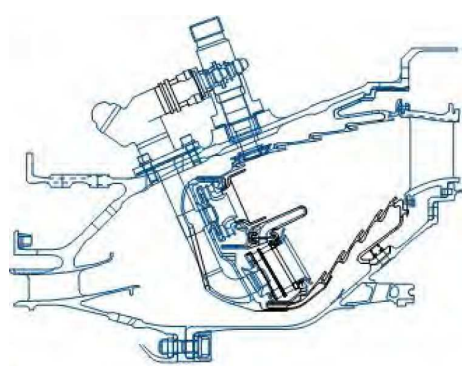

(a) CFM56 DAC

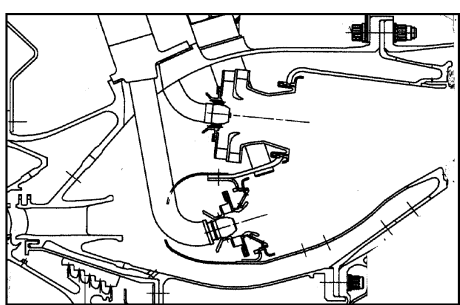

(b) CLEAN combustor

Figure 1.5: Dual Annular Combustors

Nevertheless, dual annular combustors are subject to a deterioration of $\mathrm{CO}$ and uHC emission levels in comparison with Single Annular Combustors (SAC). Furthermore, this concept leads necessarily to an increase in weight and complexity of the system, involving large surfaces that demand a significant amount of cooling air, not compatible with the requirements in terms of air available for the lean combustion, hence limiting the potential improvement in $\mathrm{NO}_{x}$ emissions.

These are the reasons why all the engine manufacturers are focusing on the development of single annular combustors. With the aim of providing the benefits of fuel staging, GE has developed the TAPS injector (Twin 
Annular Premix System) [14. The TAPS combustor concept is a lean burn system where each fuel injector contains a center pilot and concentric outer main as shown in Figure 1.6. The central pilot flame is a rich burn configuration, where $100 \%$ of the fuel is directed at starting and low power operation. At higher power approximately $70 \%$ of the air flow passes through the injector and most of the fuel is injected through the main swirler, thus providing a lean combustion.

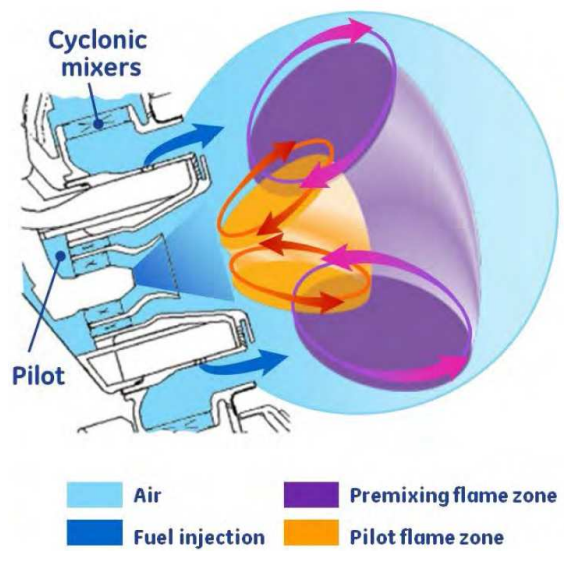

Figure 1.6: TAPS Fuel Injection Concept [14]

The development of TAPS combustors started in 1995 as a GE/NASA emissions reduction technology programme and they are currently used in the GEnx engines entered in service in 2010. A further improvement of this concept is represented by the so-called TAPS II and it will be applied in the LEAP engine family that will enter into service in 2016.

At the moment, General Electric represents the only manufacturer to produce lean combustors for civil aeroengines, nevertheless many efforts have been spent by the competitors to develop this kind of technology. Large investments have already been made in Europe through R\&T programmes and collaborations to reduce the negative impact of aircraft use on the environment. Research is therefore providing the technologies 
to improve the performance of existing engine components.

Past research programmes have already identified concepts and technologies to contribute to these goals. The engine industry has set-up a roadmap to find the optimal solutions for all the different applications (i.e. long/short range - big/small aircraft) identifying different innovative configurations for the core engine. Also for what concerns the combustor, several concepts for the implementation of lean combustion were investigated. Fuel preparation plays a key role with a view to obtaining a homogeneous air-fuel mixture and avoiding the stoichiometric conditions. This process is deeply influenced by the operating conditions, e.g. air temperature and pressure, especially considering the use of liquid fuel, that would require the atomization and the complete evaporation of the droplets. Due to non-linear effects, the scaling of fuel preparation devices proves to be critical, therefore, it appears straightforward that different technologies must be exploited depending the size and cycle of the engine. At this purpose three concepts of injection systems were developed in the framework of the NEWAC project with the aim of achieving a drastic reduction of $\mathrm{NO}_{x}$ emissions: LPP, PERM and LDI (see Figg. 1.7 and 1.8 .

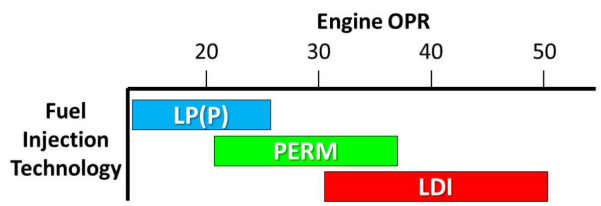

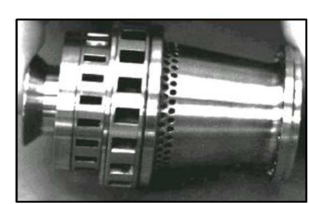

$L P(P)$

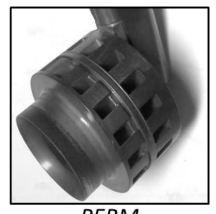

PERM

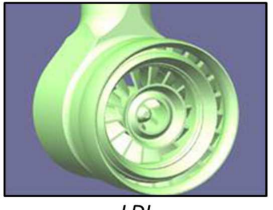

LDI

Figure 1.7: Low $\mathrm{NO}_{x}$ injectors developed in the context of NEWAC project 


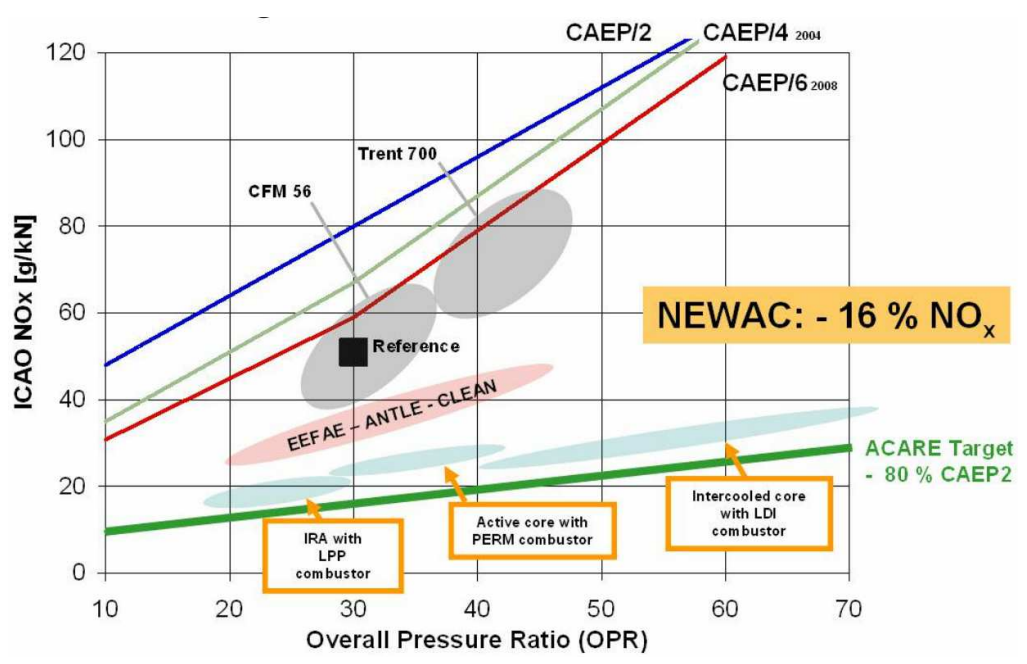

Figure 1.8: $N O_{x}$ emission reduction target of NEWAC EU project [15]

- LPP: with the aim of achieving the drastic $\mathrm{NO}_{x}$ abatement indicated by ACARE, the best way is to create a lean uniform mixture between vaporized fuel and fresh air before its introduction into the combustor. This is accomplished through the Lean Premixed Prevaporised (LPP) injector concept, which is well adapted to the low OPR cycle engine, because the low pressure minimizes the risk of auto-ignition inside the injection system.

- PERM: Premixing and prevaporization do not represent a feasible solution on medium/high OPR engines due to the inherent risk for auto-ignition and flashbacks that may lead to integrity problems of both the burners and the combustor. With the purpose of overcoming this issue, the PERM injector (Partially Evaporated and Rapidly Mixing) has been developed aiming at achieving a partial evaporation of the fuel within the swirler and the rapid mixing within the combustor, so as to minimize the presence of stoichiometric conditions in the mixture. The injection of the pilot 
fuel is accomplished in the inner swirler, whereas the main part is sustained through the formation of a liquid fuel on a lip and the subsequent breakup into droplets.

- LDI: In the light of the flashback issues ascribable to LPP injectors at elevated pressure and temperature, the LDI (Lean Direct Injection) concept has been proposed. In such combustors the fuel is directly injected in the combustion zone, where it is simultaneously vaporized, mixed and burned. The biggest challenge is represented by the design of the injector, that must provide effective atomization and rapid mixing of fuel and air to avoid the occurrence of local stoichiometric conditions. Furthermore, to ensure a stable operating range, fuel staging is arranged within the injector. A concentric pilot nozzle is injecting fuel into a recirculating flow to establish low-power flame stability. The main fuel is injected into low residence time regions to enable high-power low nitrogen oxide emissions. The pilot/main fuel split follows an optimized schedule throughout the engine operating range.

The transition towards lean burn combustion involves technological issues that must be addressed in order to achieve a successful application. Compared to RQL injectors, the aerodynamics of these devices has to be completely reviewed; moreover, the FAR required to obtain a drastic abatement in $\mathrm{NO}_{x}$ emissions leads to serious concerns that must be addressed during the development of ULN combustors, such as ignition, altitude re-light and weak extinction stability.

Lean combustors are inherently subject to thermo-acoustic instabilities, a phenomenon generated by the coupling between combustor acoustic modes and unsteady heat release. The instability can be initiated by small perturbations in the mean flow and, depending on operating conditions and combustor geometry, can alter the flame and heat release characteristics leading to oscillations in acoustic pressure field, resulting in both performance and structural degradation. This phenomenon is more pronounced for combustors operated at leaner conditions and char- 
acterized by shorter axial extension, which shifts the instabilities to higher frequencies. For these reasons, particular attention must be paid in the evaluation of the characteristic times related to these processes and at providing acoustic damping.

The thermal management of the hot gas path components represents another key aspect that is heavily affected by the increased mass flow rate dedicated to fuel preparation. Since the available amount of coolant is decreased of about $50 \%$, the target of maintaining the metal temperature below the melting point requires necessarily greater efforts, involving the identification and application of more effective cooling schemes and their local optimization depending on actual flow conditions and working regime. Furthermore, the interaction between flame and coolant must be controlled, in order to avoid the quenching of the reactions and the further leaning of the mixture, that would increase the production of unburnt species and under certain circumstances to the blow out of the flame. 


\section{Chapter 2}

\section{Heat Transfer in Gas Turbine Combustors}

A drastic reduction in $\mathrm{NO}_{x}$ emission entails necessarily significant technological advancements for what concerns the design of the cooling systems. As previously described in Chapter 1, improvements of RQL concept focus on the limitation of the employment of film cooling in the primary zone, requiring the application of different cooling schemes such as tiles or double wall liners. Lean burn combustors, on the contrary, are not subject to particular issues related to the presence of film cooling, but they are characterized by a significant reduction of air available for cooling. Moreover, the improvement of engine performance through the increase of pressure ratio results in an higher coolant temperature, reducing the cooling capacity of the air. Considering all these aspects, it is clear that the development of more efficient cooling technologies is mandatory.

After a brief introduction concerning the heat transfer phenomena occurring in combustion chambers, this chapter will provide an overview on the cooling techniques employed, with a particular focus on full coverage film cooling, or effusion cooling. 


\subsection{Flow and temperature field in aero-engine com- bustors}

Gas turbine combustors are devices that operate at high energy intensity, in the order of $1 \mathrm{GW} / \mathrm{m}^{3}$, ensuring at the same time the operativeness for up to 20000 hours before undergoing significant maintenance [16]. The trend towards more and more compact combustors imposes significant efforts in designing swirled injectors with increased hot gas recirculating capability and more effective cooling systems to manage the metal temperature below the material melting point.

The comprehension of the flow field characteristics is crucial in order to understand the issues represented by the design of a well-performing cooling scheme. Modern combustors employ swirled injectors to achieve the flame stabilization through the vortex breakdown mechanism. The swirling flow is characterized by a sudden expansion, determining a highly three-dimensional flow field that interacts with the walls, making the estimation on the thermal heat loads an hard task.

Several works dealt with the characterization of the flow structures generated by swirlers: an extensive review of swirling flow is given by Lilley [17. Among the outcomes of the work, a critical value of 0.6 for the swirl number $\left(S_{N}\right)$ was identified, which results in strong radial and axial pressure gradients nearby the nozzle exit, resulting in a central recirculation zone and other typical flow structures, such as the vortex breakdown [18] and the precessing vortex core (PVC).

Both numerical and experimental works were aimed at the characterization of these flow structures: Syred et al. [19] used a two component laser anemometry system to measure the flow field due to the interaction of PVC and the reverse flow zone in the exhaust of a swirl burner. $\mathrm{Vu}$ and Gouldin 20] reported hot-wire measurements in an axisymmetric combustor: in the RCZ, tangential velocities were found to be very small while levels of turbulence and dissipation rate were very high. Rhode et al. 21] performed mean flow field studies in axisymmetric combustor geometries with swirl. Various flow field configurations with different side 
wall and swirl vane angles were investigated to characterize the time-mean streamlines, recirculation zone, and regions of high turbulence. The length and the width of the recirculation zone was found to increase with swirl vane angle until a critical angle was reached, after which any further increase in swirl shortened the length but further increased the width. The major effect of side wall expansion angle was to shorten the corner recirculation region, with no major effects on the central recirculation. Ferrell et al. 22] reported experiments with five-hole pitot probe velocity measurements and flow visualization.

More recently, Spencer et al. 23, 24] performed PIV, LDA and hot wire measurements supported with LES calculations in an annular full scale three-sector isothermal model of a gas turbine combustor: they gave details to a better understanding of the processes occurring in the combustor such as the annular jet impingement and the interaction of the swirled flow with the primary jets. Gnirb and Tropea 25] performed PIV and concentration measurements using POD analysis on a gas-turbine model composed by 5 swirlers in planar arrangement. In addition to the mean flow field inside the model, measurements highlight the concentration of mass flow injected through a dilution hole.

More of the works reported above deal with flow field analysis of injector developed for traditional diffusive combustor, however in the last years with the growing interest on development and optimization of lean injector several authors have carried out flow field measurements on the novel LDI and PERM injectors. Due to the fact that most of the air (up to $70 \%$ ) passes through the injection system, the flow field is characterized by high intensive recirculation zones and strong interactions with the liner surface.

Marinov et al. 26] and Kern et al. 27] studied the flow field produced by a PERM injector in a cylindrical combustion chamber by means of numerical simulations and LDA measurements: they characterised the shape and the intensity of the RCZ generated by the injector and found that only LES approach is able to predict high frequency dominant coherent structure such as PVC. In addition they highlighted the difference 
between reactive and non reactive flow field: the reactive case reduces the axial length of the Inner Recirculation Zone (IRZ), and the near-wall flow field seems to be subjected to more intensive acceleration and velocity magnitude (Fig. 2.1).

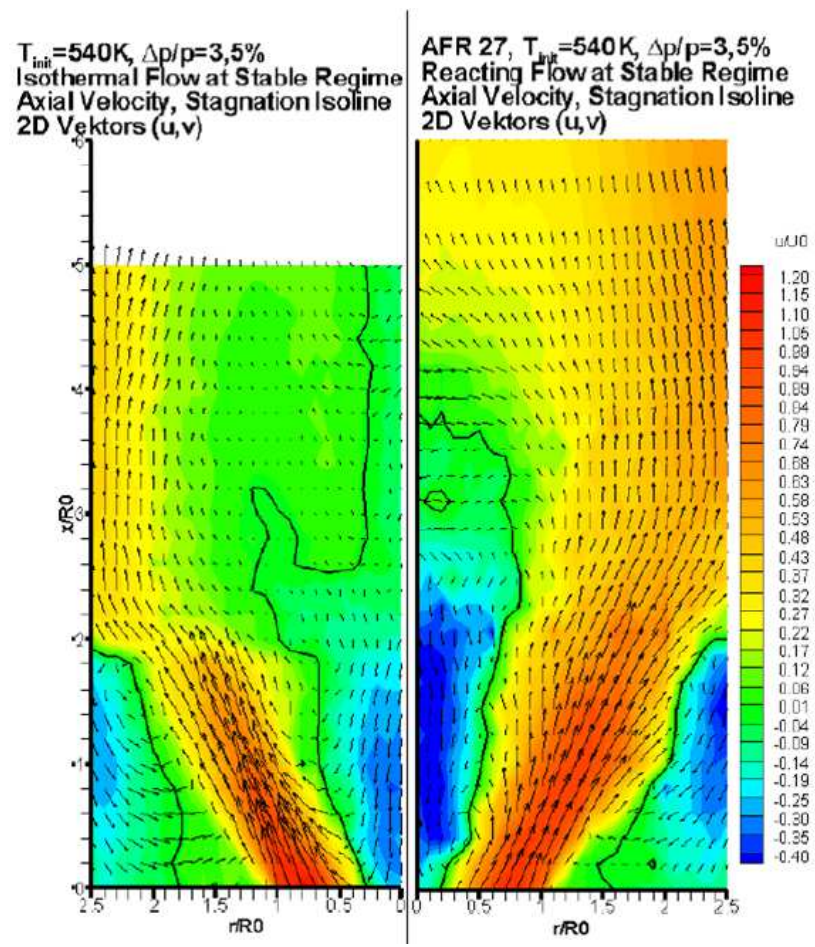

Figure 2.1: Flow field measurement of a PERM injector in isothermal (left) and reacting/stable condition (right) [26]

Wurm et al. 28] carried out the investigation of swirled combustion chamber flows in a three sector scaled test facility equipped with LDI injector at isothermal condition (Fig. 2.2. PIV measurements downstream of the central burner highlighted the identification of characteristic near-wall effects and provided proper information for the design of effective cooling system. 


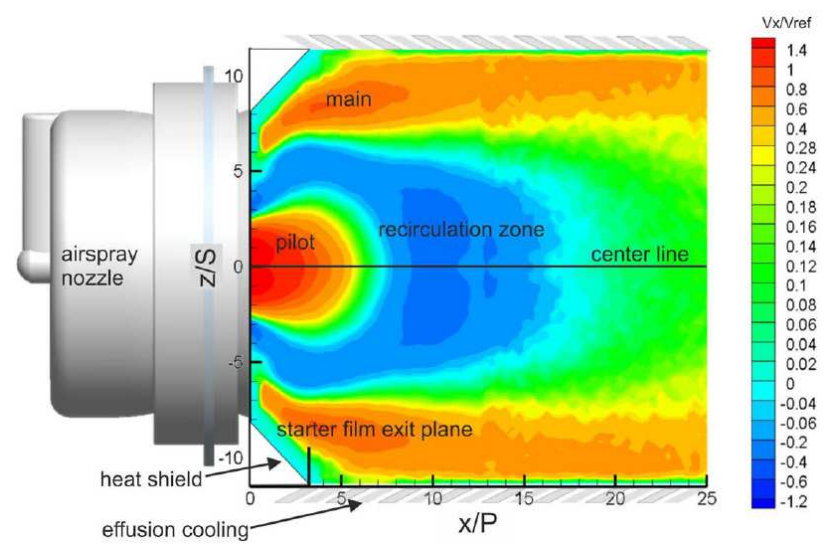

Figure 2.2: Flow field measurement of a LDI injector in isothermal condition [28]

\subsection{Techniques for liner cooling}

Even though the liners are subject low mechanical stresses, if compared to other engine components such as the turbine blades, they must withstand to high temperature and thermal gradients, that undermine their durability. Moreover, lean combustor are prone to thermo-acoustics instabilities which can match the eigenmodes of the structure and, without control through acoustic damping, can provide further stress to the liners.

To ensure a sufficient life of such components, metal temperature must be limited below an acceptable values. The definition of an acceptable level is rather arbitrary and benefits from the continuous evolution in the metallurgy field. According to Lefebvre [7, for nichel-based alloys the maximum operating temperature should not exceed $1300 \mathrm{~K}$, with flame temperatures usually above $2000 \mathrm{~K}$. Therefore, a major contributions to liner durability must be ascribed to the cooling system.

Forced convection inside the annuli is the basic method to cool the liner. Nevertheless, mere forced convection is not suitable for the high temperature within the combustor flame tube. As a consequence, different approaches were studied with the aim of increasing the heat transfer 
surfaces and coefficients on the coolant side. The promotion of turbulence through ribs, usually employed for blade cooling, allows an increase in heat transfer coefficients of 2-3 times with respect to smooth surfaces. Studies on the application of different cooling arrangements are available in literature, such as impingement cooling or alternative configurations obtained combining different techniques.

The most widely used technique to control the liner temperature is film cooling. It is implemented by injecting relatively cold air bled from the annulus on liner inner side through holes or slot, thus creating a protective film that shields the liner from a direct contact with hot gases.

In its basic design, film cooling makes use of a number of annular 2D slots injecting the coolant axially along liner hot surface; the film cooling is gradually destroyed downstream the injection point by the turbulent mixing with hot gas flow, so the normal practice is to repeat a succession of slot along the liner length. Mechanical robustness and low weight are the mayor advantages of such a technique which has found widespread application in both industrial and aircraft gas turbines. An example of slot cooling configuration is displayed in Fig. 2.3 the picture shows also the dilution holes located in some sections of the liner: these holes are necessary to complete the combustion process in a RQL combustor but they do not contribute to film cooling.

The demand for more powerful and efficient engines makes the need of developing more performing cooling systems increasingly important. According to Lefebvre [7, the main reasons are:

- Increase of $O P R$ : the increase of the overall pressure ratio allows to obtain substantial reductions in engine fuel consumption. Unfortunately, increasing the OPR raises the radiative heat transferred to the liner walls; in addition, increasing the pressure ratio causes an increase in combustor inlet temperature, thus reducing the capability of the annulus air to cool the walls.

- Increase of TIT: turbine inlet temperature directly affects the overall efficiency of the engine; therefore the necessity of increasing TIT 


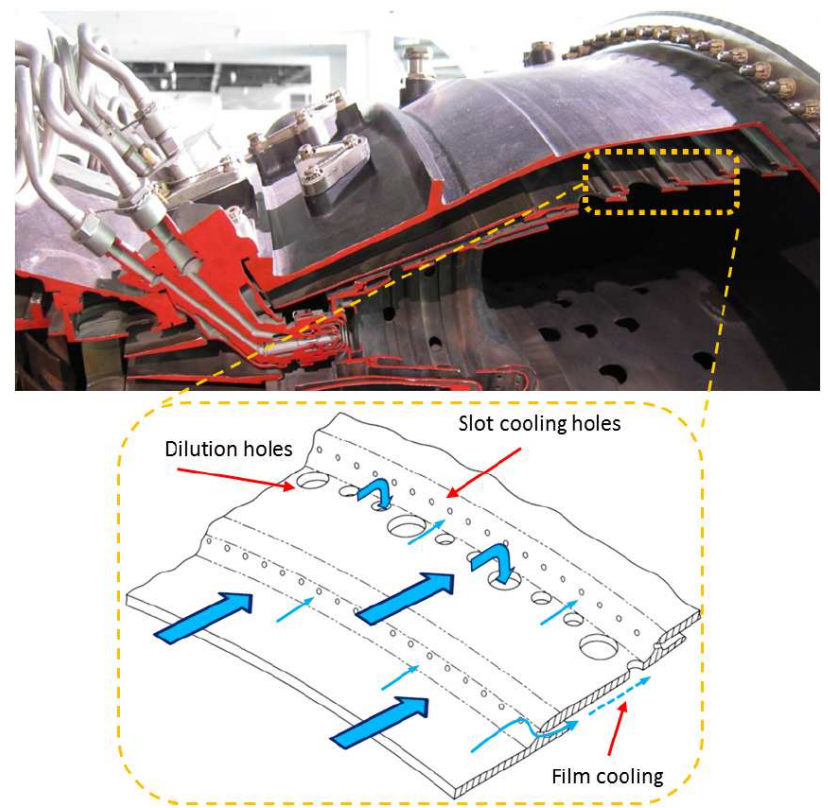

Figure 2.3: Example of liner cooling arrangement (PEWW JT9D)

calls for improvements in combustor pattern factor in order to maintain the integrity of the hot sections downstream. Since film cooling air flows along the liner wall, it makes a minor contribution to mixing. Thus, reduction in wall cooling consumption gives a direct contribution to pattern factor improvement.

- Increase of durability: as noted by Dodds and Bahr in [29], as combustor operating temperatures has been increased, component durability and life expectations have also risen and thus the engine operating time before combustor maintenance has exponentially grown.

- Introduction of new combustion technologies: regulations governing $\mathrm{NO}_{x}$ pollutant emissions have become increasingly stringent and lean burn technologies have been identified as the most promising to meet 
the new legislation limits; such technologies need to highly increase air amount necessary to combustion, at the expense of air available for liner-wall cooling. This trend driven by was the knowledge that reductions in film-cooling air are highly beneficial in lowering the emissions of carbon monoxide and unburned hydrocarbons.

Following these consideration, the need to find out alternative solutions to the typical slot cooling configuration has become mandatory.

In addition to cooling performance, other aspects should be taken into consideration during the choice of the optimal solution, such as pressure losses, increased weight and manufacturing costs. Gerendàs et al. [30, for example, describes the development phases of the dual annular combustor BR715, pointing out the advantages related to single skin effusion cooling if compared to cooling rings (higher cooling effectiveness), transpiration cooling sheets (easier local cooling adaptation for hot spot treatment) and tiles on a carrier liner (lower weight). Effusion cooling, consisting of an array of closely spaced discrete film-cooling holes, can be considered a interesting compromise between full coverage film cooling, widely used in blades and nozzles, and transpiration cooling [31, the best efficient cooling solution based on porous materials, but subjected to reliability issues, e.g. structural disadvantages and pores occlusion, that at the moment do not allow the implementation in combustor applications.

\subsubsection{Effusion Cooling}

Effusion cooling is realized drilling a dense array of closely spaced cylindrical holes with a small diameter in the order ot few tenths of millimeter (see Fig. 2.4). The hole have small injection angles (usually $30^{\circ}$ ), even though it is possible to achieve angles up to $10^{\circ}$ to obtain a better coolant protection in the proximity of the wall, in spite of increased manufacturing costs.

The intrinsic features of this cooling technique determine several favourable characteristics:

- the dense array of holes provides an uniform protecting layer, ensured 


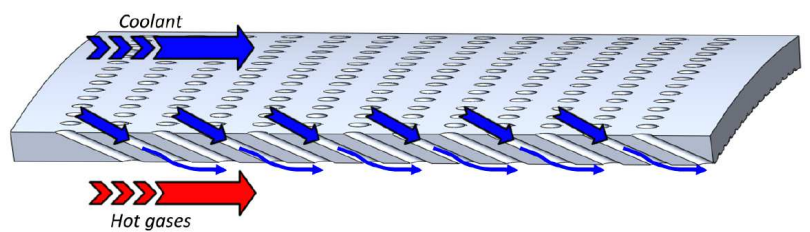

Figure 2.4: Effusion cooled liner

by the continuous coolant injection that gradually increases due to the film superposition

- holes are characterized by a high length-to-diameter ratio $(L / D)$ that involves a significant heat sink effect, as a consequence of the force convection with high heat transfer surface [32, 33, 34]

- the mass bleeding on the coolant side provides a significant increase in HTC, which can contribute up to $30 \%$ to total cooling effectiveness 30 .

Despite the higher manufacturing costs and the lower film protection if compared to 2D slots, at least in the first part of the liner (where superposition is not fully developed), this technique allows to overcome some technological issues given by classical cooling arrangements, making it very attractive for lean burn combustors. First of all, it ensures a very efficient cooling performance with a reduced coolant consumption with respect to 2D slots [35] (see Fig. 2.5.

Moreover, effusion systems permit to avoid the protection degradation downstream of the injection point: in typical 2D slot cooling a large amount of air is injected through each slot to ensure a sufficient survival of coolant without using an excessive number of injection points. This leads to an over-cooling in the regions immediately downstream to the slot, involving dangerous thermal gradients that could generate cracks formation, whereas effusion cooling provides an uniform protection and heat removal within the liner.

Before going into the details of this cooling technique, the next section reports a brief description of the "jet in cress flow" (JCF) phenomenon, 


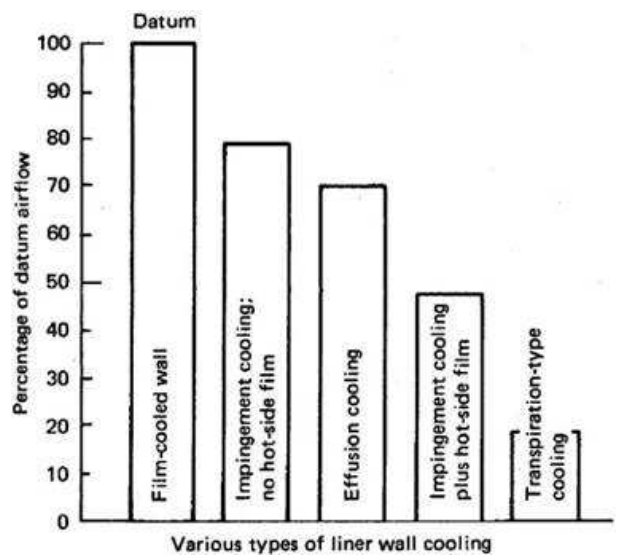

Figure 2.5: Potential coolant related to the application of different cooling techniques

followed by an overview of the main parameters that affect film cooling with special attention on those related to effusion systems.

\subsubsection{Geometrical parameters}

Literature studies on effusion cooling are usually carried out on simplified configurations representative of multi-perforated plates. Such geometries can be described, making reference to Fig. 2.6 in terms of hole diameter $(D)$ and length $(L)$, stream-wise and span-wise pitches $\left(S_{x}\right.$ and $\left.S_{y}\right)$, inclination and compound angle $(\alpha$ and $\gamma)$.

Geometrical features that are usually employed in gas turbine cooling to enhance the cooling performance, such as fan shaped holes or laidback holes, are not adapted for effusion holes: despite the achievable advantages in fact, their implementation would entail a not affordable manufacturing cost. The selection of the effusion geometrical parameters are thus dictated by the trade-off between manufacturing costs and cooling performance. 

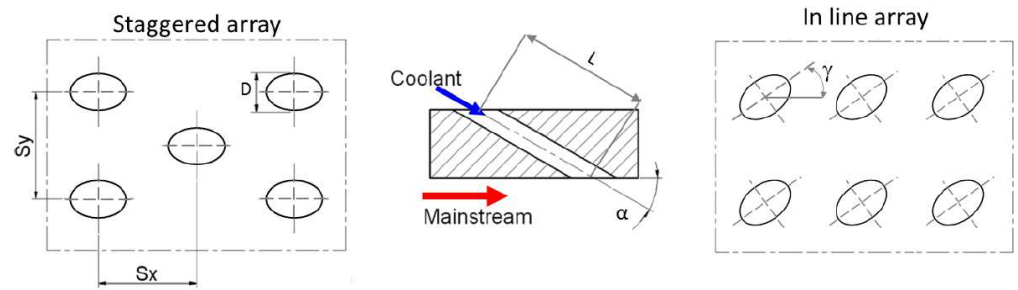

Figure 2.6: Main geometrical parameters for effusion cooling arrangements

\subsubsection{Operating parameters}

Several fluid dynamic parameters were introduced in literature to characterize the interaction between main stream and coolant, such as:

Velocity Ratio:

$$
V R=\frac{v_{c}}{v_{g}}
$$

Density Ratio:

$$
D R=\frac{\rho_{c}}{\rho_{g}}
$$

Blowing Ratio:

$$
B R=\frac{(\rho v)_{c}}{(\rho v)_{g}}
$$

Momentum Flux Ratio:

$$
I=\frac{\left(\rho v^{2}\right)_{c}}{\left(\rho v^{2}\right)_{g}}
$$

Velocity ratio determines, as reported by Han et al. 31, the particular injection regime of cylindrical holes: for $V R>0.8$ the jet lift-off from the surface occurs, starting to penetrate within the main flow.

In modern aero-engine combustors the working conditions are highly variable, not only because of the different working conditions during the mission cycle, characterized by significant changes in pressure, temperature and mass flow rate, but also along the combustor liners: in the first part of the liner the coolant in injected in a relatively cold recirculating region, 
whereas moving towards the combustor exit the hot gas is subject to an acceleration due to the convergence of the flame tube. For these reasons, general guidelines are difficult to be drawn, since the potential range can vary for blowing ratio to $B R \approx 1-7$, whereas for density ratio to $D R \approx 1.5-3.0$.

Another useful parameter is represented by the jet Reynolds number, which controls the heat transfer and the pressure losses within the hole:

$$
R e_{j}=\frac{(\rho v)_{j} D}{\mu}
$$

\subsubsection{Performance parameters}

Several parameters were proposed in literature to assess the performance of cooling schemes. It could be useful to start from Newton's law of cooling for the convective heat flux, reviewed by Goldstein [36]:

$$
q=H T C_{\operatorname{main}}\left(T_{w}-T_{a w}\right)
$$

where $H T C_{\text {main }}$ represents the heat transfer coefficient on a film cooled surface. The driving potential for convective heat transfer is the difference between wall temperature $T_{w}$ and adiabatic wall temperature $T_{a w}$. This parameter, introduced by Goldstein, would be obtained for an adiabatic surface and its choice as the reference temperature yields, at least for a constant property flow, to an $H T C$ value independent on the temperature difference.

Adiabatic wall temperature is usually presented in non-dimensional form as the adiabatic effectiveness $\eta_{a w}$ :

$$
\eta_{a w}=\frac{T_{\text {main }}-T_{a w}}{T_{\text {main }}-T_{c o o l}}
$$

where $T_{\text {main }}$ is the mainstream total temperature and $T_{\text {cool }}$ is the temperature of flow injected from the cooling hole.

Another important parameter to be taken into account for the evaluation of the convective heat load in presence of film cooling is the above mentioned $H T C_{\text {main }}$ and can deeply differ from the reference heat trans- 
fer coefficient in absence of film cooling $\left(H T C_{0}\right)$. Being the final aim of a cooling system the reduction of the heat transfer to the surface, which is dependent on both adiabatic effectiveness and heat transfer coefficient, both $\eta_{a w}$ and $H T C_{m a i n}$ are required to determine the actual reduction of overall heat load (refer also to Kelly and Bogard [37). At this purpose, Sen et al. [38] suggest to use the Net Heat Flux Reduction parameter (NHFR) to quantify the global heat transfer achieved on a cooled surface. It is defined as the ratio between the heat flux reduction due to film cooling and heat flux taking place without cooling $\left(q_{0}\right)$ :

$$
N H F R=\frac{q-q_{0}}{q_{0}}=1-\frac{H T C_{\text {main }}}{H T C_{0}}\left(1-\eta_{a w} \theta\right)
$$

where $\theta$ is the non-dimensional temperature, defined as:

$$
\theta=\frac{T_{\text {main }}-T_{\text {cool }}}{T_{\text {main }}-T_{w}}
$$

The ultimate goal of a well performing cooling system is thus to increase NHFR values: this target is achieved through a reduction in $H T C_{\text {main }} / H T C_{0}$ and an enhancement in $\eta_{a w}$. It is worth point out that increased mixing within the boundary layer due to coolant injection cause a significant increase in the mainstream heat transfer coefficient 37, therefore adiabatic effectiveness should be high enough to compensate for this HTC augmentation, otherwise the heat transfer reduction is not achieved.

Despite the validity of the previous considerations, NHFR is not properly representative for effusion cooling systems, since its definition (Eq. 2.8 does not explicitly take into account for the heat sink within the perforation and on the back side of the plate. To overcome this aspect, the parameter the overall effectiveness $\eta_{o v}$ is introduced in literature to indicate the overall cooling performance of a cooling system:

$$
\eta_{o v}=\frac{T_{\text {main }}-T_{w}}{T_{\text {main }}-T_{\text {cool }}}
$$

As it is possible to notice, the definition considers the actual wall temperature, hence taking into account all the contributions to wall 
cooling, including film protection and heat sink effect. For this reason, this parameter is suitable to give information about the overall performance of an effusion system.

\subsubsection{Literature Review of Effusion Cooling for Combus- tor Liners}

Studies on film cooling started several decades ago, as demonstrated by the studies performed by many authors since the 60's. a specific summary of the investigations aimed at understanding the influence of geometrical and operative parameters on this phenomenon is beyond the scope of this short review. Attention will be focused to relevant past studies concerning effusion cooling for combustors liner.

Effusion cooling has been subject of attention from the 80's [39]. In the early works, investigations have been performed with the aim of investigating the impact of geometric features and operating conditions on the thermal performance on effusion cooling plates. Martiny et al. 40. evaluated row by row adiabatic effectiveness and performed flow visualizations on an effusion cooled plate with a very shallow injection angle of $17^{\circ}$; their study included also different flow patterns for the investigated blowing ratios, varied from 0.5 to 4.0 . They noticed that in the starting region of a cooling film at blowing ratio of 0.5 jets remain attached to the wall (Fig. 2.7a) and for higher values of blowing parameter a closed cooling film is prevented by the jets lifting-off from the surface (Fig. 2.7b). Nevertheless, even in cases of complete penetration, cooling benefits, in terms of adiabatic effectiveness, are still visible: they are ascribable to the massive coolant injection, which increases row by row and reduces main flow temperature in the mixing zone.

The same facilities and test samples were employed by Martiny et al. 41] while developing an analytical model for the calculation of wall temperature of an effusion cooling array: by means of a parametric study, they investigated the dependencies of dimensionless quantities on the overall effectiveness.

Many studies on full coverage film cooling have been focused on 


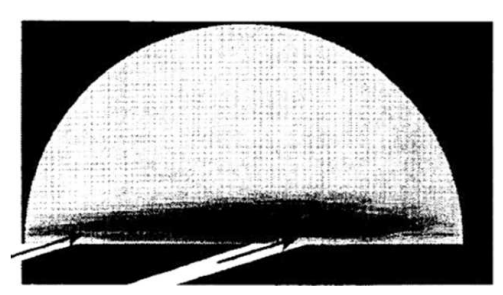

(a) Flow pattern $B R=0.5$

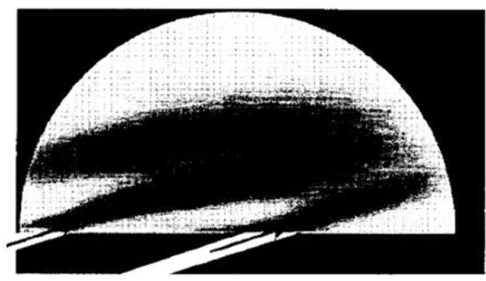

(b) Flow pattern $B R=4.0$

Figure 2.7: Schlieren flow traces, $2^{\text {nd }}$ and $3^{\text {rd }}$ effusion row observed 40]

measuring or estimating the film effectiveness generated by coolant jets and the heat transfer of effusion cooling. A detailed experimental investigation on hot side heat transfer associated to an effusion solution is the one due to Kumada et al. [42] ; even though not recent, the work presents a precious effort in correlating the wall heat transfer coefficient variation with the jets fluid dynamics.

Scrittore et al. [43] investigated an effusion cooling scheme through flow field measurements and adiabatic wall temperatures. Of particular interest was the considered range of blowing ratio, from 3.2 to 5.0 , which is closer to the one that could be found in typical applications for combustor liners; furthermore, authors studied a cooling scheme close to a real engine one, that contained twenty rows of cooling holes with equally spaced holes in both stream-wise and span-wise directions $\left(S_{x} / D=S_{y} / D=4.0\right)$. They measured adiabatic effectiveness of this full coverage scheme, finding the attainment of a fully developed effectiveness region at the $15^{\text {th }}$ row and a very low effect of blowing ratio on cooling performance. They also observed a fundamental independence of penetration height for the cooling hole rows on the momentumflux ratio for the range that was considered at the fully-developed condition. Finally, they also confirmed the hypotheses proposed by Schulz 44]: measuring velocity planes around the holes, jet separation just downstream of the hole trailing edge was highlighted, in addition to the presence of a counter-rotating vortex pair (CVP) which generates hot mainstream flow entrainment toward the 
surface at the plate leading edge. Downstream, however, this effect serves to mix the accumulated coolant flow and provides a more uniform surface temperature across the pitch. The cooling effectiveness of the film layer increases asymptotically at the same rate for both blowing ratios, with only little improvement for the higher blowing ratio with respect to the lower blowing ratio.

Scrittore et al. 45] performed also a flow field investigation to better understand the influence of dilution jets on effusion film development; results showed how the coolant was transported from the film cooling layer in the near wall region to the free-stream and downstream from the dilution jets. Furthermore, the presence of the dilution jet caused an high increase of turbulence level: such an effect resulted in an increased spreading of the film-cooling jets. Increasing dilution blowing ratio resulted in a degradation of film cooling. Examples of such results are shown in Fig. 2.8

Metzger et al. 46] studied the variation of heat transfer coefficient for full-coverage film cooling scheme with normal holes, founding an augmentation of $20-25 \%$ in the local heat transfer with blowing ratio 0.1 and 0.2. Martinez-Botas and Yuen 47. measured heat transfer coefficient and adiabatic effectiveness for a variety of flat plate geometries to test the influence of the injection angle by varying blowing ratio from 0.33 to 2.0. They measured the variation of the heat transfer coefficient $h$ with respect to a reference case $h_{0}$; results show that there is a maximum of $h / h_{0}$ close to the hole and further downstream with highest heat transfer augmentation for $30^{\circ}$ injection angle.

Kelly and Bogard 37. investigated an array of $90^{\circ}$ normal holes and found that the largest values for $h / h_{0}$ occur immediately downstream of the film cooling holes and the levels of $h / h_{0}$ are similar for the first 9 rows. They explained that this could be due to an increase in the local turbulence levels immediately downstream of the holes, created by the interaction between the cooling jet and the mainstream flow. Another reason could be the formation of a new thermal boundary layer immediately downstream of the cooling jets. None of the previous studies investigates the effect that 


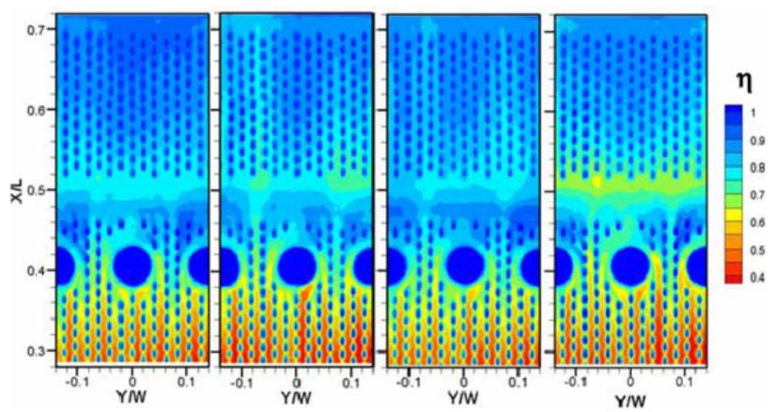

(a) Adiabatic effectiveness maps

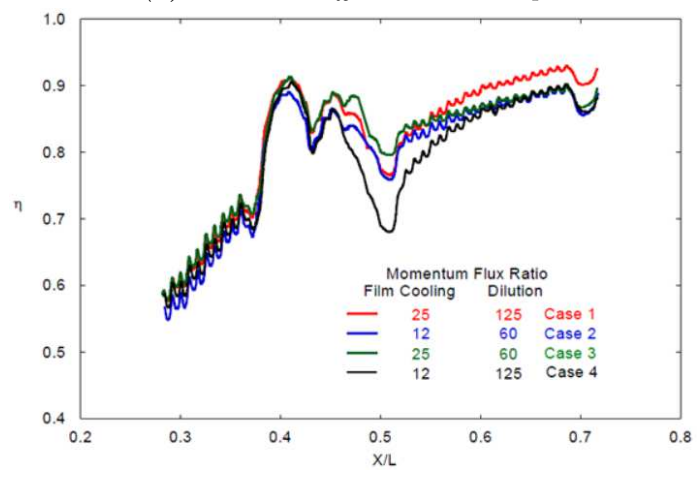

(b) Spanwise averaged effectiveness

Figure 2.8: Film cooling effectiveness near combustor dilution hole [45]

a high blowing ratio has on adiabatic effectiveness, heat transfer coefficient, and Net Heat Flux Reduction. As reported by the authors, increases in adiabatic effectiveness due to high blowing ratios could potentially be replaced by an increase in heat transfer coefficient due to high mainstream turbulence.

Facchini et al. 48 estimated the heat transfer coefficient at variable blowing ratios on the same real engine cooling scheme to evaluate the combined effects of slot, effusion, and a large dilution hole; they found that an increase in VR makes jets become more and more penetrating in the main flow and they behave as an obstacle against film development, 
lowering the effectiveness values. On the contrary, they found that high BR values enhance the heat transfer phenomenon: in fact, the high jet velocity that draws hot gases from mainstream flow on the liner surface alters the local boundary layer on the effusion surface causing an increase of HTC. Andreini et al. 49] performed a CFD analysis on the same test article, simulating the behaviour of the cooling system both with air and $\mathrm{CO}_{2}$. Numerical results show that the entity of local heat transfer enhancement in the proximity of effusion holes exit is due to gas-jets interaction and that it mainly depends on effusion velocity ratio; furthermore, a comparison between results obtained with air and with $\mathrm{CO}_{2}$ as coolant pointed out the effects of density ratio, showing the opportunity to scale the increase in heat transfer coefficient with effusion jets velocity ratio.

An exhaustive study on the cooling system preliminary design for modern lean burn combustors was provided by Ceccherini [50]: a onedimensional analysis on combustor liner cooling system was carried out, highlighting the ability to improve the performance of effusion. Furthermore, CFD investigations were performed as well as experimental surveys in order to give more details of the behaviour of effusion cooling and its practical implementation in engine-like cooling schemes.

More recently Ligrani et al. [51] presented film effectiveness and heat transfer results for full coverage film cooling arrangements with streamwise pressure gradient; they studied the effect of the blowing ratio and the influence of dense and sparse hole arrays on the thermal effectiveness, giving an extensive overview of the behaviour of this system subjected to several operating conditions. Experiments under conditions of very high freestream turbulence up to $25 \%$ were conducted by Martin and Thorpe [52] on an effusion test plate with angled hole array and staggered pattern, varying blowing ratios from 0.3 to 1.5 . They found that when freestream turbulence is increased the spatially averaged effectiveness can increase by as much as $85 \%$ at $\mathrm{BR}=1.4$ due to the span-wise spreading and turbulent transport of coolant back towards the plate surface.

Despite many studies deal with the investigation of the effusion cooling performance, most of them were conducted by using air as coolant and 
main flow, precluding the possibility to point out the effects of density ratio between the two flows. Density ratio is, however, a key parameter for the design of a liner cooling system, mainly because of the actual large temperature difference between coolant and burned gases inside the core of the combustor. Ekkad et al. 53, 54 measured effectiveness and heat transfer coefficient distribution over a flat surface with one row of injection holes inclined stream-wise at $35^{\circ}$ for several blowing ratios and compound angles; tests were carried out by using air and carbon dioxide as coolant, finding that both heat transfer and effectiveness increase with blowing ratio. They also pointed out the effects of density ratio, showing how these effects are more evident with increasing the compound angle and the momentum flux ratio. This experimental survey was, however, oriented for turbine blade applications rather than combustors.

More recently, Lin et al. [55, 56] investigated both experimentally and numerically adiabatic film cooling effectiveness of four different $30^{\circ}$ inclined multi-hole film cooling configurations; the survey, which was specific for combustor liner applications, was performed by using a mixture of air and $\mathrm{CO}_{2}$ as coolant, but it was mainly focused on studying the influence of hole geometrical parameters and blowing ratio on film cooling rather than on the effects of density ratio.

Moving to more representative configurations, Facchini et al. 557. investigated also the influence of a recirculating area in the mainstream on the same geometry: they highlight that the presence of the recirculation impedes the creation of the film layer, leading to a general reduction of the effectiveness but without having significant effects on the heat transfer coefficient. Despite the step is able to reproduce a RCZ in the first part of the liner, the flow field can not be considered fully representative of a lean aero-engine combustor.

The impact of a realistic flow field on film cooling have been the focus of attention of recent research studies with the aim of understanding the actual thermal performance of cooling schemes at engine-like conditions. For a deeper insight concerning this topic it is possible to refer to Chapter 3 



\section{Chapter 3}

\section{CFD Investigation of Combustor Cooling}

Technical literature regarding the characterization of effusion cooling devices mainly deals with simplified gas side flow conditions (i.e. uniform flow). Nevertheless lean burn combustion burners are characterized by high swirling flows, generating the typical vortex breakdown, which strongly affects the near wall flow along both liners and dome. Thus a reliable estimate of liner thermal protection can not neglect the presence of a complex 3D flow field interacting with the coolant jets 58.

To authors' knowledge, some of the early studies taking into account swirled flows are the recent investigations at Karlsruhe Institute of Technology [58, 59]. These papers report the experimental investigations performed on a linear test rig composed by three nozzles and liners cooled by means of starter film and effusion cooling. In [58, the focus was on the impact of the swirled flow on film cooling effectiveness as well as of the heat shield geometry on the starter film penetration. The increased pressure drop across the liner resulting in higher film protection does not significantly influence the starter film area. The study conducted in 59. is instead devoted to the definition of a dimensionless cooling efficiency to compare the performance of effusion cooling schemes in combination 
with different air-spray nozzle designs. The results showed how the use of different nozzles strongly influences the cooling system performance due to the variation of local flow conditions.

Another experimental investigation was conducted by Andreini et al. 60, which report flow field and heat transfer measurements on a scaled combustor with a realistic swirling flow generated by a lean-burn injector. The campaign was carried out in order to evaluate the influence of the combustor pressure drop and the coolant consumption through the slot cooling system. The activity pointed out a strong interaction between swirled flow and liner surface due to the central recirculation zone, characterized by high velocity gradients in the near-wall region and responsible for a significant heat transfer augmentation, which nevertheless is not deeply affected by the combustor pressure drop. The injection of air through the slot inhibits the heat transfer and shifts downstream the peak location due to the stagnation point, even though the coolant is quickly lifted up by the corner vortex, hence providing minimal protection on the liner.

Bearing in mind the complexity and the cost associated to the experimental investigation of these kind of applications, CFD has become a key and complementary tool to understand the phenomena involved. From a numerical perspective, an interesting attempt to characterize the effects of the swirled flow on combustor heat transfer is conducted by Patil et al. 61] which, studying an experimental test case consisting of an air spray nozzle with a swirl number of 0.7 , pointed out how increasing Reynolds number does not change the position of the peak in heat flux due to the impingement of the swirl flow on the liner whereas the intensity of the peak is reduced (as recently confirmed by Andreini et al. 60.). Another activity involving CFD is reported by Wurm et al. 62, which used RANS calculation to estimate flow field and metal temperature distribution on the above mentioned test rig. In particular, the simulations were employed to test optimised configurations of heat shield and starter film, aiming at providing a more uniform coolant injection.

Due to the well-known limitations of RANS approach, usually point- 
ing out an underestimation of mixing, and the increase in computation resources, LES simulations are becoming nowadays more popular. The issues related to the accurate specification of the turbulent boundary conditions at the inlets and the spatial resolution requirements in the near wall region still represent critical challenges for an extensive use of LES approach to test cases with realistic conditions in terms of Reynolds number. With the perspective of an exploitation to industrial applications, mainly characterized by wall bounded configurations, hybrid RANS-LES models have been proposed to overcome these issues. Detached Eddy Simulation (DES) 63 achieves the switch between RANS and LES by a comparison of the turbulent length scale with the grid spacing, whereas Scale Adaptive Simulation (SAS) 64, 65] consists in an improved URANS formulation that provides an LES-like behaviour in unsteady regions through the introduction of the von Karman length-scale into the turbulence scale equation. Other approaches are based on zonal modelling and the LES is resolved only in predefined zones where unsteady results are required.

A numerical assessment of hybrid RANS-LES models on a non-reactive flow of a gas turbine model combustor is reported by Widenhorn et al. 66], who compared the time-averaged velocity components and turbulent fluctuations (RMS) obtained with URANS SST, SST-SAS and SSTDES simulations. The best agreement with the LDA measurements was obtained by the SST-DES model, even though it requires a significant mesh refinement in order to increase the portion of the combustion chamber resolved with LES, whereas on equal refinement of the computational grid the SST-SAS model provides more accurate results. More recently, Patil and Tafti [67] simulated flow and convective heat transfer in a gas turbine can combustor exploiting a zonal two layer model. The results pointed out a good agreement in predicting both trends and values of the heat transfer coefficient distribution and a significant improvement compared with previous RANS calculations 61.

Here below are described the main findings of numerical investigations aimed at deepening the knowledge concerning combustor cooling, with particular attention to effusion cooling and the effects of its interaction 
with the swirling flow on the cooling performance. At this purpose, thee test cases at increasing representativeness:

- Flat effusion cooled plates: validation of the CFD tool against experimental measurements of $\eta_{a d}$, with particular reference to an advanced RANS model that accounts for turbulence anisotropy.

- Tubular effusion cooled combustor: exploitation of the validated numerical approach to investigate the impact of swirling flow-liner cooling interaction on the $\eta_{a d}$ distribution.

- Annular slot cooled combustor: validation of hybrid RANS-LES models for the prediction of Nusselt distribution.

Before moving into details of these analyses, the next section will provide an introduction to the issues related to the numerical simulation of film cooling, as well as the approach proposed in literature to overcome the deficiencies of standard RANS models.

\subsection{Numerical study of film cooling}

The injection of a jet in a transverse flow, or cross-flow, gives rise to a highly complex turbulent flow field dominated by many large scale, coherent flow structures which develop as a consequence of the interaction of the two streams 68. Despite the interest and the efforts devoted to the computational analysis of the near wall development of the Jet in Cross-Flow (JCF) scheme, suitable CFD procedures capable of giving satisfactory predictions have not been standardized yet. Even though DNS and LES have recently expanded the knowledge regarding the flow structures developing within the JCF [69, 70, 71], these techniques cannot still be considered as a valuable tool for design purposes or in the simulation of complex cooling systems, due to their high computational cost and complexity. Classical steady RANS approach exploiting eddy viscosity turbulence modelling, on the other side, typically shows common weaknesses in over-predicting the jet penetration and systematically underestimating the lateral spreading [72, 73]. 


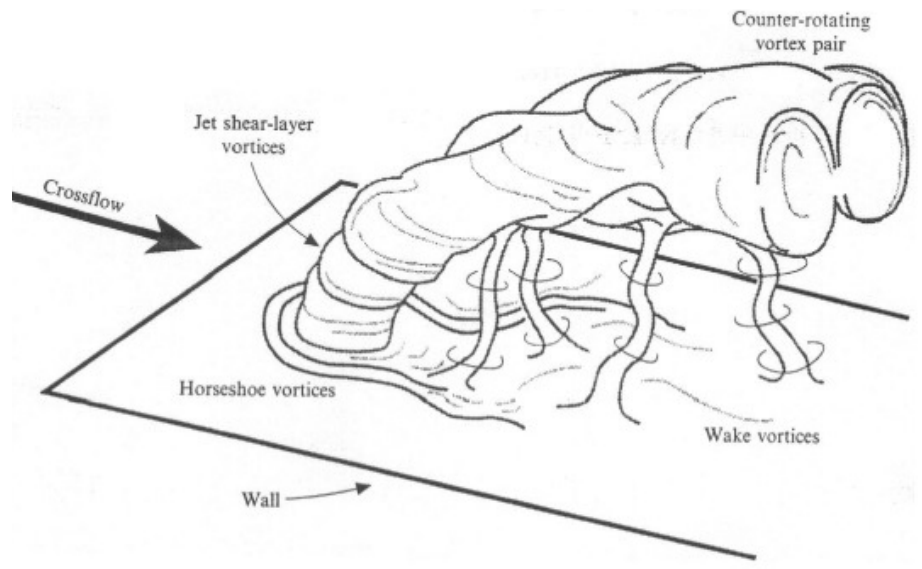

Figure 3.1: Sketch of the experimental test rig

In fact, for a jet in cross-flow the hypothesis of isotropic turbulence ceases to be applicable in the mixing zone. The normal to the wall fluctuation is damped by the wall itself while the stream and spanwise directed fluctuations are influenced by the interaction with the jet. The macroscopic effect is an enlarged lateral diffusion compared to the other two main directions [74. Despite their theoretical capability to partly model turbulence anisotropy, the Reynolds Stress models do not usually significantly improve the overall accuracy of JCF mixing. Another explanation of the mentioned deficiencies is, in fact, the intrinsic unsteadiness of JCF which cannot be modelled through steady RANS 75 .

Regardless of the cited numerical issues, the availability of accurate and reliable turbulence models for JCF computation with RANS approach is still a very challenging activity to help engineers in the design and optimization of real cooling systems. In the last years several works have dealt with this objective. A pioneering approach in this field is the concept of directional eddy viscosity early introduced by Bergeles et al. [76] to take into account the anisotropy of turbulent field in JCF. The 
idea is to use a tensorial definition of eddy viscosity, where the terms responsible for jet lateral diffusion are augmented through a correction factor determined by higher order simulations (DNS data). This concept has been developed more recently by Azzi and Lakehal [77. with a detailed methodology to include the directional eddy viscosity in a Two Layer $k-\epsilon$ model. This methodology was implemented and applied on literature test cases [74] and more recently to actual combustor liner cooling geometries 78, 79, 80, 81. A first attempt to extend the concept of directional eddy viscosity to other turbulence models was carried out by Cottin et al. 82 where the idea of Bergeles was implemented in a $k-\omega$ SST model with a benchmarking on a typical combustor liner effusion cooling geometry. Further contribution to this family of models comes from [83, where in the tensorial definition of eddy viscosity a set of general purpose shape functions obtained by higher order computations are used.

The main drawback of this family of models is the necessity to establish the orientation of coordinate system axis with respect to holes geometry and therefore they can be strictly applied just to flat plate like geometries. Moreover such formulations are derived for steady state analysis hence are valid for the mean fields only.

\subsection{Flat effusion cooling plate}

\subsubsection{Introduction}

The aim of this work is to benchmark the standard $k-\omega$ SST turbulence model and its modified version with the anisotropic correction over a wide range of working conditions. Low and high blowing ratio conditions corresponding respectively to the mass addiction and penetration regimes 84] are investigated. The effects of jet superposition is studied as well, considering a 18 row staggered array of holes. Orthogonal and slanted perforations are analysed, as well as the effect of different turbulence levels on cooling performance. All the results presented refer to adiabatic computations and they are compared with available experimental data for $\mathrm{DR}=1.0$ and 1.5 tests. 


\subsubsection{Investigated test case}

This numerical analysis is based on equivalent geometries and flow conditions as those employed in a parallel experimental investigation performing Pressure Sensitive Paint measurements to evaluate purely adiabatic effectiveness [85]. Among the different arrays considered in the experimental campaign, only two plates, characterized by a different hole inclination angle $\alpha$, are considered in this work; most relevant geometric features are summarized in Table 3.1

\begin{tabular}{ccccccc}
\hline Name & $D[\mathrm{~mm}]$ & Rows & $L / D$ & $\alpha$ & $s_{x} / D$ & $s_{z} / D$ \\
\hline G2 & 1.50 & 18 & 6.25 & 30 & 9.15 & 7.37 \\
G7 & 1.50 & 18 & 3.13 & 90 & 9.15 & 7.37 \\
\hline
\end{tabular}

Table 3.1: Test plate geometry

The numerical set up aims at reproducing the main characteristics of the experimental rig (depicted in Fig. 3.2 ) such as the feeding duct shape of the hot and coolant gas and a turbulence generator grid able to provide two different levels of turbulence intensity, low $(T u=1.6 \%)$ and high $(T u=17 \%)$. Furthermore, each plate has been tested with several values of blowing ratio $(B R=1.0,2.0,3.0)$ and density ratio $(D R=1.0,1.5)$. In order to generate oxygen partial pressure gradient necessary for the PSP based technique, the coolant is fed with nitrogen while the mainstream consisted of air. The test is conducted at almost isothermal condition thus the effective density ratio is virtually 1 .

The full test matrix of the simulations performed in the present work is summarised in Table 3.2 . The experimental data set was also exploited to perform a computational model sensitivity which was conducted on G2 at $B R=2$ chosen as the reference case.

\subsubsection{Numerical setup}

3D CFD RANS calculations have been considered in this study, referring to the Navier-Stokes solver ANSYS ${ }^{\circledR}$ CFX v14. The mainstream 


\begin{tabular}{ccccccc}
\hline Name & $\alpha[\mathrm{deg}]$ & $D R[-]$ & $T u[\%]$ & \multicolumn{3}{c}{$B R[-]$} \\
\hline \multirow{3}{*}{ G2 } & \multirow{2}{*}{30} & 1.0 & 1.6 & 1 & 2 & 3 \\
& & & 17.7 & 1 & 2 & 3 \\
& & 1.5 & 17.7 & 1 & 2 & 3 \\
\hline \multirow{2}{*}{ G7 } & \multirow{2}{*}{90} & 1.0 & 17.7 & 1 & 2 & 3 \\
& & 1.5 & 17.7 & 1 & 2 & 3 \\
\hline
\end{tabular}

Table 3.2: Test matrix of performed simulations

boundary conditions have been assigned in terms of total pressure, total temperature and turbulence quantities at the main inlet, mass flow rate and total temperature are specified at the coolant inlet and mass flow rate was fixed at the outlet (Figure 3.3.

Compressibility effects have been taken into account and a High Resolution advection scheme has been used. Energy equation was solved in terms of total energy and viscous heating effects have been accounted for. Turbulence was modelled by means of the $k-\omega$ SST turbulence model, both in its original formulation made available by the CFD solver and with an algebraic anisotropic correction suited to overcome typical RANS modelling failures for film cooling flows as described in details up ahead. Employed near wall treatment uses an automatic blending between Wall Function and Wall Integration approach on the basis of $y^{+}$

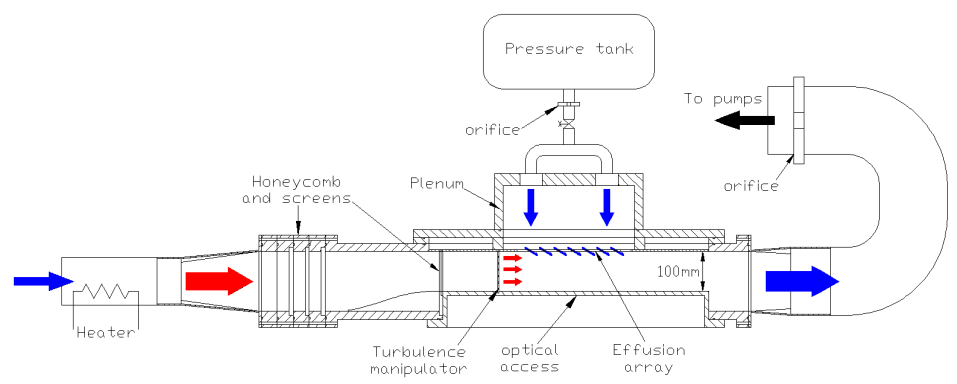

Figure 3.2: Sketch of the experimental test rig 
value. For the cases presented in this work where $\max \left(y^{+}\right)<1$, the Low Reynolds formulation is always recovered.

Injections of both air and nitrogen were modelled with identical gas properties assuming ideal gas behaviour. From a dimensional analysis the thermal field in totally driven by convection, so the effect of buoyancy was neglected both for $D R=1.0$ and $D R=1.5$. In order to track the coolant distribution, a transport equation for an additional passive scalar EffCool representing coolant concentration, holding the value 1 at the plenum inlet and 0 at the mainstream inlet, was solved. The value of its kinematic diffusivity was specified to guarantee a Le=1 with the aim of fully respecting the mass-energy transfer analogy.

ANSYS $^{\circledR}$ ICEM-CFD has been used to generate hybrid computational grids (tetrahedral with 15 layers of prisms close to the wall). In order to reduce the computational cost, it has been decided to take advantage of the symmetry condition offered by the geometry (see Figure 3.3). In this way, only half hole has been modelled for each row.

The solution convergence has been assessed by monitoring that RMS residuals fell below a prescribed value of $1 e 6$ and verifying that the averaged value of $\eta_{a d}$ on the plate reached a steady-state.

\subsubsection{Algebraic anisotropic correction}

Numerical details of the implemented corrections are reported in this section. The basic idea is to calculate the Reynolds Stress tensor as:

$$
-\rho \overline{u_{i}^{\prime} u_{j}^{\prime}}=\mu_{t, i j}\left(\frac{\partial U_{i}}{\partial x_{j}}+\frac{\partial U_{j}}{\partial x_{i}}-\frac{2}{3} \delta_{i j} \frac{\partial U_{k}}{\partial x_{k}}\right),
$$

where turbulent viscosity is augmented for the stream-span directions by an amplification factor $\gamma$ :

$$
\mu_{t, i j}=\left(\begin{array}{ccc}
\mu_{t} & \mu_{t} & \gamma \mu_{t} \\
\mu_{t} & \mu_{t} & \mu_{t} \\
\gamma \mu_{t} & \mu_{t} & \mu_{t}
\end{array}\right)
$$

The value for the anisotropic correction $\gamma$ follows the correlation in Eq. 


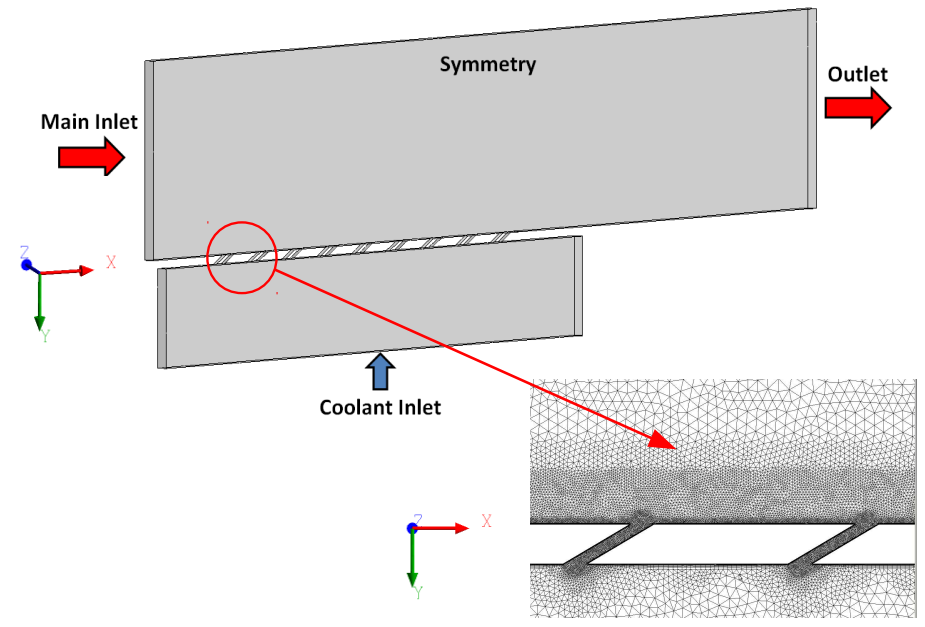

Figure 3.3: Sketch of the numerical domain and particular of the mesh

3.3 bounded to the upper and lower limits of 60 and 4.5, for $y^{\star}<1.5$ and outside the buffer layer respectively [77. The lower bound corresponds to the asymptotic value at the edge of the boundary layer as measured by Kaszeta and Simon [86, while the upper bound inside the viscous sub-layer is suggested by Azzi and Lakehal [77 to prevent numerical instabilities arising in case of small $y^{\star}$ due to negative values of $\gamma$.

$$
\gamma=\frac{1000 y^{\star 0.42}}{2.682 y^{\star 2}-5.463}
$$

This function was proposed by [87] to match DNS simulations of turbulent flat plate by 88 , in which:

$$
y^{\star}=0.00442 R e_{y}^{2}+0.294 R e_{y}+0.545 .
$$

The proposed correction is applied to the domain of the evolving jet only, thus isotropic formulation is recovered inside the coolant plenum, within the perforations and outside the boundary layer in the main duct. 


\subsubsection{Source Terms}

The above described anisotropic modification was firstly inserted in the framework of $k-\omega$ SST model by Cottin et al. [82 and subsequently tested by the authors in 89 . The anisotropic correction is introduced by means of additional source terms (conventionally taken on the right hand side of the conservation equations) modelling turbulent flux modification in the momentum, energy, turbulence and passive scalar transport equations. For the momentum equation, source terms can be directly derived from Eq 3.1 while for the transport equation of a passive scalar $\phi$, following the Generalized Gradient Diffusion Hypothesis in the modelling of turbulent diffusive fluxes, result in:

$$
-\rho \overline{u_{i}^{\prime} \phi}=\mu_{t, i j} \frac{\partial \phi}{\partial x_{j}} .
$$

Furthermore spurious terms involving cross-products, such as those in the turbulent kinetic energy production or the viscous heating terms, need to be corrected; final formulation of source terms added on the RHS of each conservation equation are reported in Eq3.6

$$
\begin{gathered}
\mathcal{S}_{u}=(\gamma-1) \mu_{t} \frac{1}{2}\left(\frac{\partial u}{\partial z}+\frac{\partial w}{\partial x}\right) \\
\mathcal{S}_{w}=(\gamma-1) \mu_{t} \frac{1}{2}\left(\frac{\partial w}{\partial x}+\frac{\partial u}{\partial z}\right) \\
\mathcal{S}_{h}=(\gamma-1)\left[\frac{\mu_{t}}{P r_{t}} \frac{\partial^{2} h}{\partial z^{2}}+\mu_{t}\left(\frac{\partial u}{\partial z}+\frac{\partial w}{\partial x}\right)^{2}\right] \\
\mathcal{S}_{k}=(\gamma-1)\left[\mu_{t} \alpha_{k} \frac{\partial^{2} k}{\partial z^{2}}+\mu_{t}\left(\frac{\partial u}{\partial z}+\frac{\partial w}{\partial x}\right)^{2}\right] \\
\mathcal{S}_{\phi}=(\gamma-1)\left[\frac{\mu_{t}}{S c_{t}} \frac{\partial^{2} \phi}{\partial z^{2}}\right]
\end{gathered}
$$

\subsubsection{Results}

\subsubsection{Mesh sensitivity analysis}

It is well known that mesh refinement plays a central role in film cooling simulations. For this purpose, a comprehensive mesh sensitivity 
analysis has been performed on the reference case in order to find a cost-effective grid resolution able to correctly capture local concentration gradients at an affordable computational cost.

Four grids have been tested in this analysis: the first mesh (Mesh A) counts 2.5 million of elements, the second one (Mesh B) has been generated reducing of $40 \%$ the global scale factor and hence increasing the number of elements up to 5.0 millions of elements. Last two grids (Mesh $\mathrm{C}$ and $\mathrm{D}$ ) included further refinements in the mixing region and within the holes, resulting in 7.2 and 22.4 millions of elements respectively.

The results have been reported in Fig. 3.4 , which highlights the effect of mesh refinement on spanwise averaged adiabatic effectiveness against experimental results from Andreini et al. [85. All simulations result in a strong underestimation in the first rows of holes, most likely caused, as demonstrated in the following section, by the assumption of turbulence isotropy on which standard $k-\omega$ SST model is based. Most important in this context is however to highlight the effects of the mesh refinement. Successive mesh refinements lower the spanwise averaged effectiveness at the end of the plate, which is opposite to the experimental evidence, but improve the capability to reproduce the trend of growth matching the slope of experimental curve. Last refinement from Mesh C to D, resulting in a three times higher number of elements, does not show a significant variation on the obtained wall concentrations, hence Mesh $\mathrm{C}$ has been used for the following investigations.

\subsubsection{Turbulence model sensitivity analysis}

The main effect of the above proposed anisotropic correction is a larger diffusivity in the near wall region which results in a lower coherence of the cooling jets whose shape, especially in the first rows of holes, is largely influenced by the turbulence modelling. Figure 3.5 reports a contour plot of coolant concentration on a plane normal to the streamwise direction positioned $1 D$ downstream the $9^{\text {th }}$ hole.

It is possible to note how the anisotropic model predictions result in a lower jet penetration and in a less pronounced kidney-vortex which is 


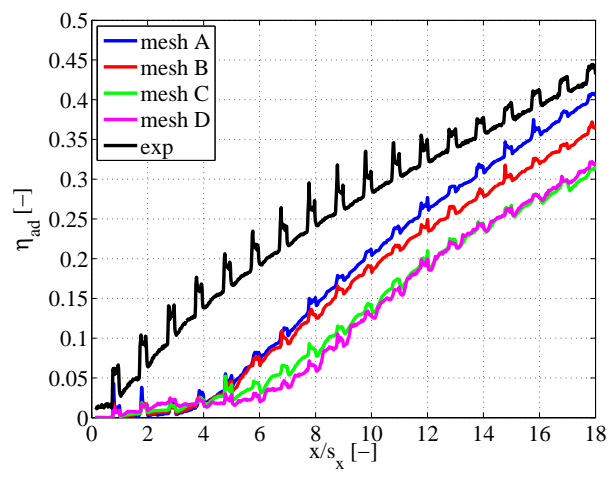

Figure 3.4: Sensitivity to mesh refinement (G2, BR=2)

responsible for the ingestion of hot gas below the jet itself. In such a way the entire region within the two symmetry planes shows a higher film protection due to a more complete merging of consecutive jets.

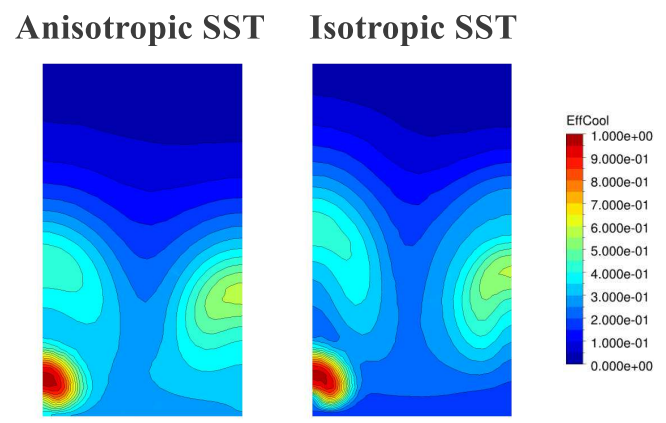

Figure 3.5: Contour plot of coolant concentration at $X / D=1$ for $9^{\text {th }}$ hole (G2, BR=2)

This effect is responsible for increased levels of cooling effectiveness especially where superposition does not play a fundamental role thus resulting in a rapid increase of averaged value able to reproduce the experimental growth rate already from the first rows of holes. Figure 
3.6a shows that, despite a slight overestimation, the agreement with the experimental curve is very good for the anisotropic SST model both in terms of concentration value and superposition effect. Compared to the isotropic model, the anisotropic model permits to reduce the discrepancy with experimental data on average by an order of magnitude as better highlighted in Figure $3.6 \mathrm{~b}$ where the relative error between spanwise averaged adiabatic effectiveness values obtained by numerical predictions and experiments is reported as a function of streamwise position. As already hinted the anisotropic correction is highly beneficial for the first rows of holes but is also capable of reducing the asymptotic error below $8 \%$ while standard isotropic formulation is assessed around $20 \%$.

The same analysis was conducted on G7, as pointed out by Figures $3.6 \mathrm{c}$ and 3.6d The two models predict equivalent levels of effectiveness with an almost constant shift of about 0.03 which makes G7 more insensitive to turbulence modelling. The anisotropic model shows the same increase of accuracy for the first 5-6 rows of holes but, since the trend of growth is overpredicted by both models, the isotropic predictions result to be closer to experimental data approaching the end of the plate. Computation of the relative error does not offer a clear estimate of which model is globally preferable thus to make the analysis more homogeneous the following simulations will be performed with the corrected turbulence model.

\subsubsection{Blowing ratio effects}

In Figure 3.7 comparisons between experimental and numerical data for $\mathrm{G} 2$ at three different $B R$ values are reported. As can be seen, numerical model well predicts spanwise averaged adiabatic effectiveness for both trend and values, especially at higher $B R$ values. CFD leads to a general overestimation of the experimental data, revealing higher peaks just downstream coolant injection as highlighted by $B R=1$ results.

Increasing the $B R$, the jets detach from the wall and the cooling effectiveness due to film protection strongly reduces, as can be seen for first rows of holes. This phenomenon takes place for $1<B R<2$ as first rows effectiveness is pretty much the same for $B R=2$ and 3 . However, 


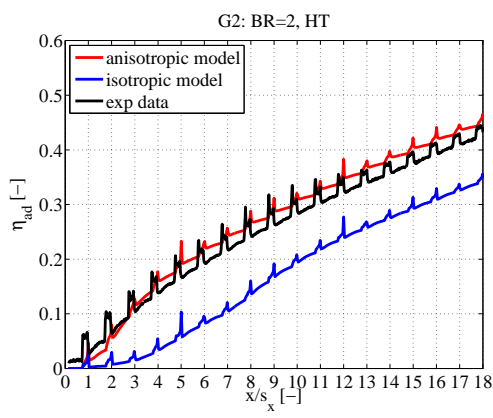

(a) Spanwise averaged $\eta_{a d}$

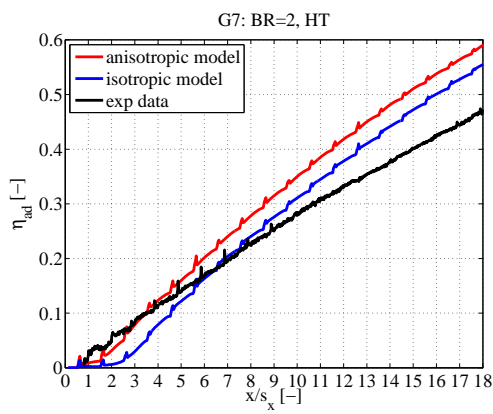

(c) Spanwise averaged $\eta_{a d}$

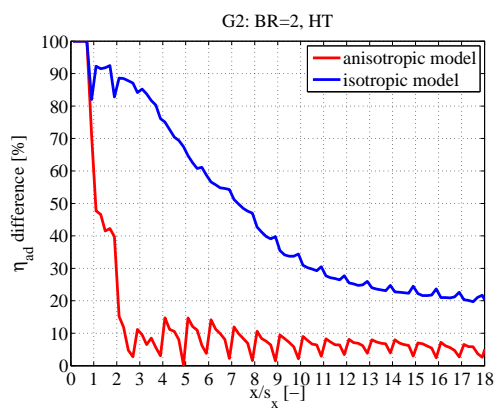

(b) Spanwise averaged $\eta_{a d}$ absolute error

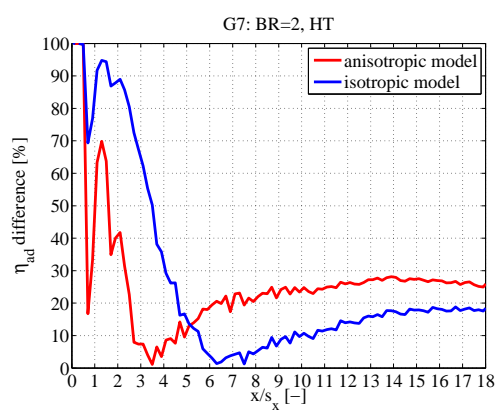

(d) Spanwise averaged $\eta_{a d}$ absolute error

Figure 3.6: Turbulence model effects on adiabatic effectiveness for both geometries.

due to the higher coolant mass flow injected, the superposition effect is larger and the plate effectiveness is enhanced downstream. Although an asymptotic condition could not be verified at any of the tested blowing ratios even for $x / s_{x}=18$, the trend of growth results to be proportional to $B R$.

This is true even more for $90^{\circ}$ holes, whose spanwise averaged $\eta_{\text {ad }}$ is plotted in Figure 3.8 , where the trends among the three investigated blowing ratios are similar also for $B R=1$ because jet is always in penetration regime. For this flow configuration where near wall effects are less 

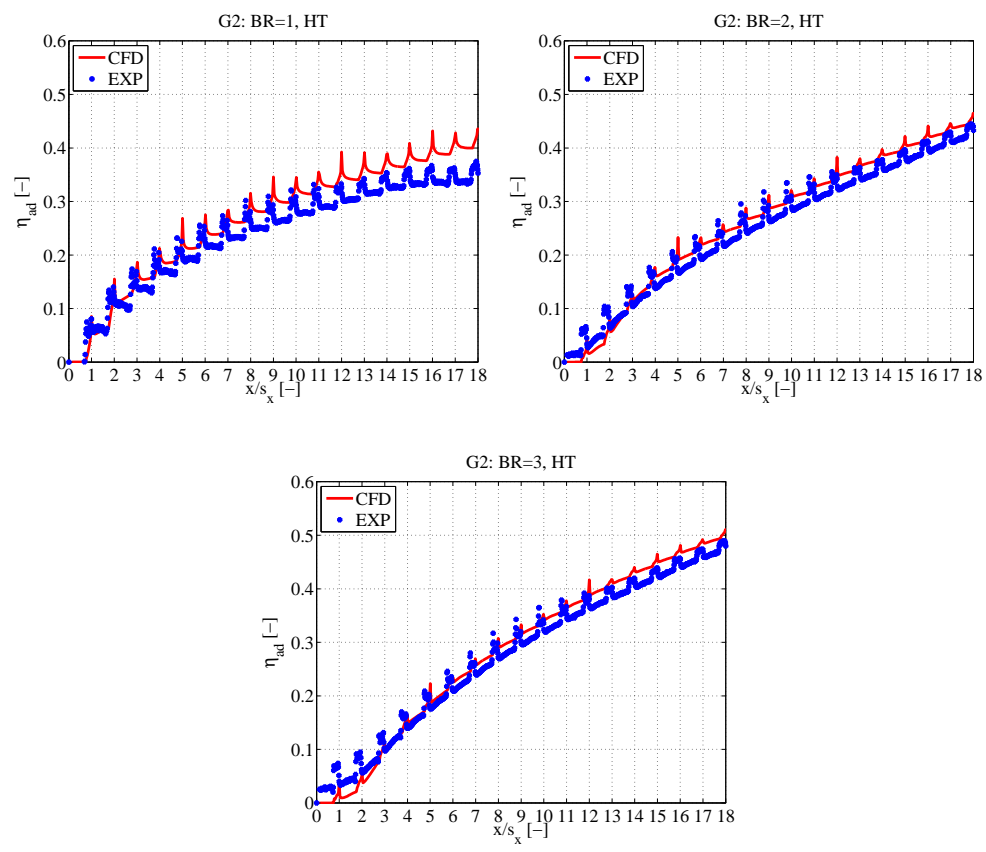

Figure 3.7: BR effects on adiabatic effectiveness (G2).

dominant, CFD predictions with the anisotropic turbulence model show a systematic overestimation of the locally averaged effectiveness and the trend of streamwise growth resulting in a shift of $\eta_{a d}$ downstream the last row of approximately 0.12 , independently on the $B R$; this corresponds to roughly $30 \%$ for $B R=1$ and to $20 \%$ a $B R=3$. As, to the authors knowledge, this is the first attempt to use such anisotropic turbulence model with orthogonal perforations, further work in the tuning of such correction is believed to provide better estimate also for normal injection flow.

In order to better understand the flow characteristics in the investigated conditions, concentration contour plot on the symmetry plane are presented in Figure 3.9. focusing on the first 3 odd rows of holes. 

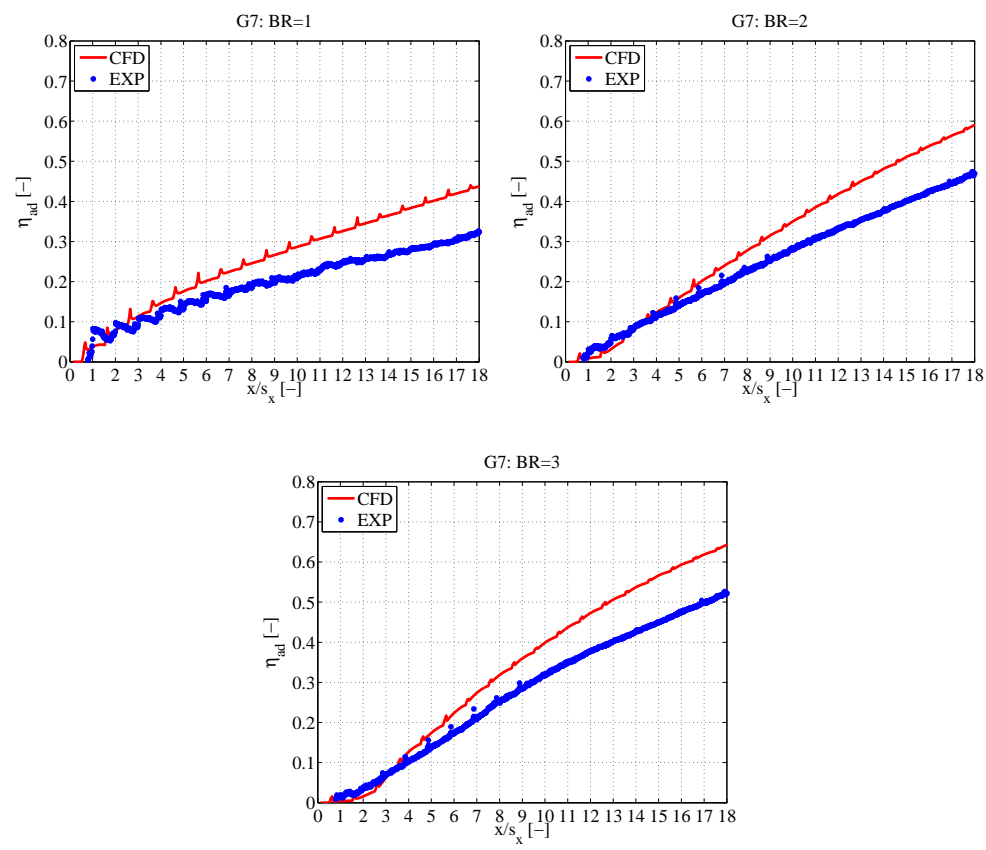

Figure 3.8: BR effects on adiabatic effectiveness ( $\left.G^{7}\right)$.

Flow conditions are largely sensitive to both $B R$ and injection angle. As already hinted mass addiction regime is reported only for $\mathrm{G} 2$ at $B R=$ 1 where jets are actually confined in a thin layer along the wall. for the $90^{\circ}$ injection instead the normal to the wall momentum is already high enough to guarantee jet penetration at least from the $3^{\text {rd }}$ row.

At the higher blowing ratio the penetration regime is achieved also by G2. Jet shape is however quite different between G2 and G7, as G7 shows a thicker wall layer affected by coolant injection and a higher streamwise diffusion of the jet, furthermore jet bending towards the wall is promoted as evidenced by the $5^{\text {th }}$ hole. 


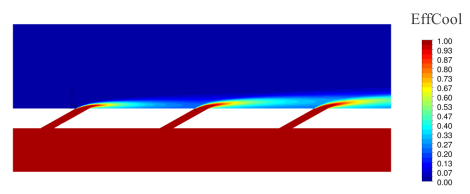

(a) G2 at $B R=1$.

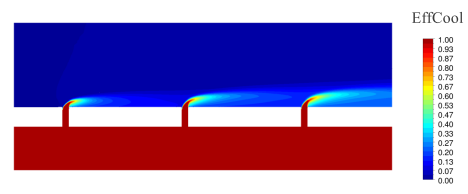

(c) $G 7$ at $B R=1$.

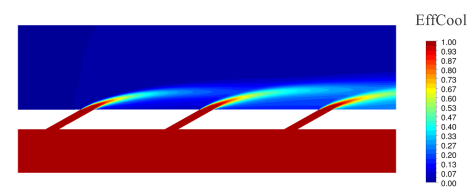

(b) G2 at $B R=3$.

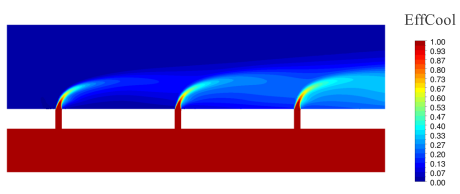

(d) $G^{7}$ at $B R=3$.

Figure 3.9: Contour plot of coolant concentration on symmetry plane for both geometries (for $1^{\text {st }}, 3^{\text {rd }}$ and $5^{\text {th }}$ rows).

\subsubsection{Inlet turbulence effects}

Figure 3.10 shows span-wise averaged adiabatic effectiveness for two levels of inlet turbulence intensity (HT and LT) with varying $B R$ for G2.

Comparing high turbulence and low turbulence intensity cases, it is possible to highlight that as turbulence intensity increases also adiabatic effectiveness increases. The enhanced level of mixing between the two flows results beneficial both in mass addiction and penetration regime because of the promoted lateral diffusion of the jet on the wall in the former case and the lower jet penetration in the latter.

Likening experiments and numerical data, it is clear that CFD is less influenced by inlet turbulence intensity especially for the high blowing ratios. Both at $B R=2$ and 3 conditions in fact, numerical results do not show the initial low levels of adiabatic effectiveness associated with LT and the two curves results to be almost coincident. This is due to the fact that numerical diffusivity is mostly driven by turbulence generated within the cooling duct which is insensitive to the inlet free turbulence intensity.

For $B R=1$ the two turbulence levels show the same effectiveness increase on the first rows but a different growth rate due to superposition resulting in a shift which remains quite constant along the plate, vice-versa 

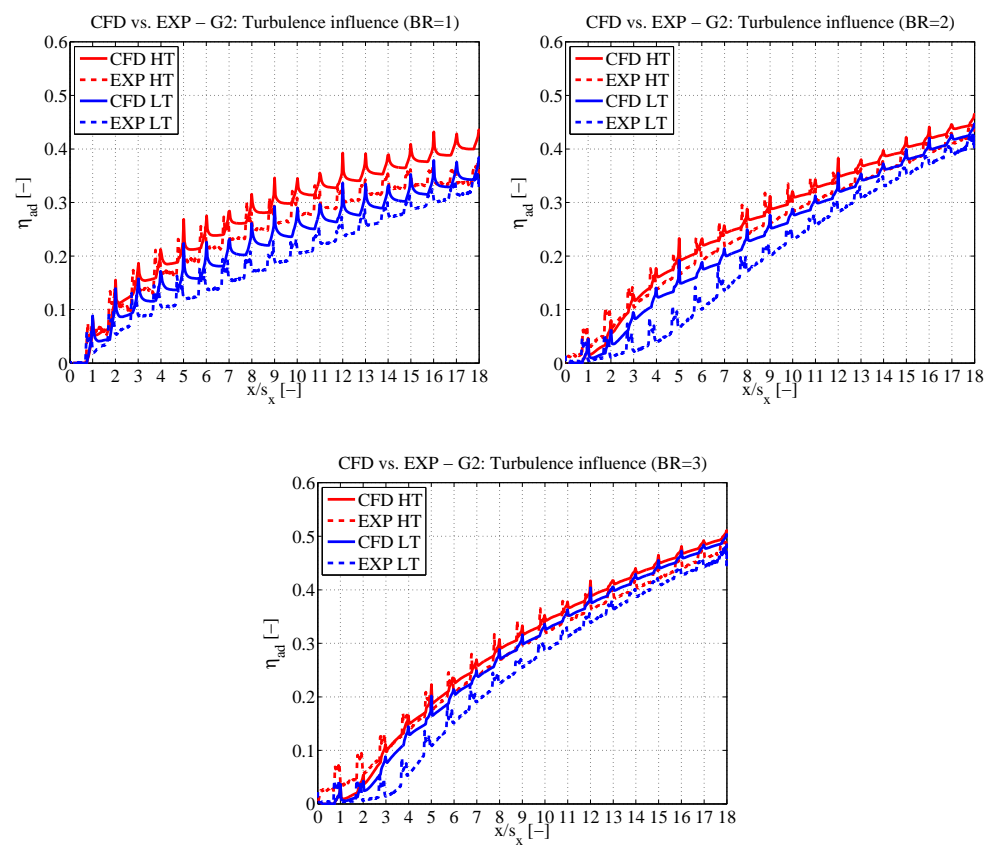

Figure 3.10: Inlet turbulence effects on adiabatic effectiveness (G2).

the experiments show similar values at the end of the plate.

\subsubsection{Density ratio effects}

Figure 3.11 reports the comparisons between experimental and numerical data for the three different $B R$ values. Concerning $D R=1$ cases, numerical model well predicts span-wise averaged adiabatic effectiveness in terms of both trend and values, especially at higher $B R$, where a relative error below $10 \%$ is achieved. The agreement slightly worsens at $B R=1$, leading to a general overestimation of $\eta_{a d}$, especially moving toward the end of the plate. As reported in 90, the anisotropic model predictions result in a lower jet penetration and in a less pronounced kidney-vortex 
which is responsible for the ingestion of hot gas below the jet itself. In such a way the entire region within the two symmetry planes shows a higher film protection due to a more complete merging of consecutive jets.

For the $D R=1.5$ cases (representing conditions that are more representative than the previous ones), looking at higher $B R$ condition, a slight worsening of the agreement can be noticed, with a gap that remains nearly constant up to the end of the plate. The same trend was not obtained for $B R=1$, in which case the overestimation becomes relevant already from the first rows. This result can be ascribed to the misprediction of the regime, as can be noticed comparing the slope of $\eta_{a d}$ curves on the first rows, which is completely different between numerical and experimental data. CFD simulation returns an increasing trend, typical of the penetration regime, whereas experiments highlight higher peak values, balanced by a sudden decay typical of mixing regime.

In order to better understand the flow characteristics in the investigated conditions, concentration contour plot on the symmetry plane are presented in Figure 3.12 focusing on the $1^{\text {st }}$ and $13^{\text {th }}$ rows of holes. Flow conditions are largely sensitive to $B R$, whereas the $D R$ has a significant effect only at low $B R$ values. As already hinted, penetration regime is reported also for $B R=1$, where, even though the jets are actually confined in a thin layer along the wall, no reattachment to wall is visible, as highlighted in Figure 3.11 by the increasing slope of the first rows.

\subsubsection{Remarks}

A CFD analysis of an effusion cooling plate composed by 18 perforations was performed exploiting steady-state RANS analysis with the aim of estimating adiabatic effectiveness. Turbulence, which is a key factor in the correct prediction of jet in cross-flow flow field, was modelled with the $k-\omega$ SST turbulence model including an algebraic correction to account for near wall anisotropy. Numerical results are compared with experimental data investigating the effect of $B R$, embracing both mass addiction and penetration regime, the influence of the injection angle, varied between $30^{\circ}$ and $90^{\circ}$, and the inlet turbulence intensity. CFD 

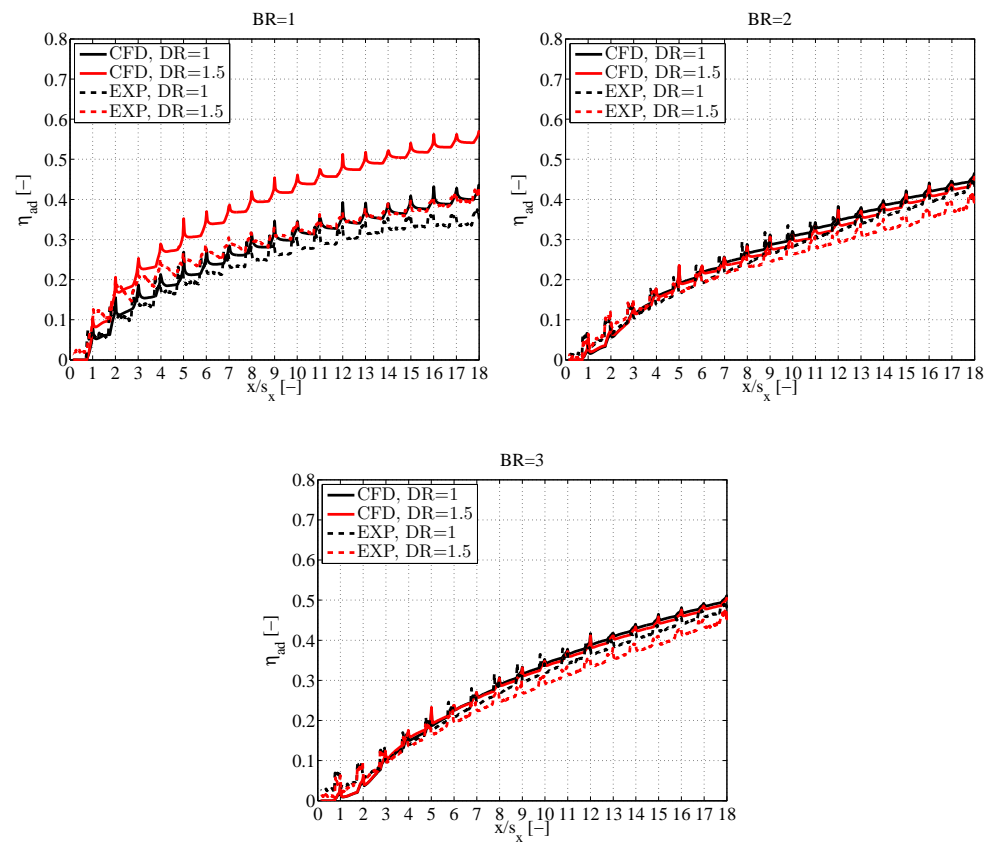

Figure 3.11: Comparisons between numerical and experimental data.

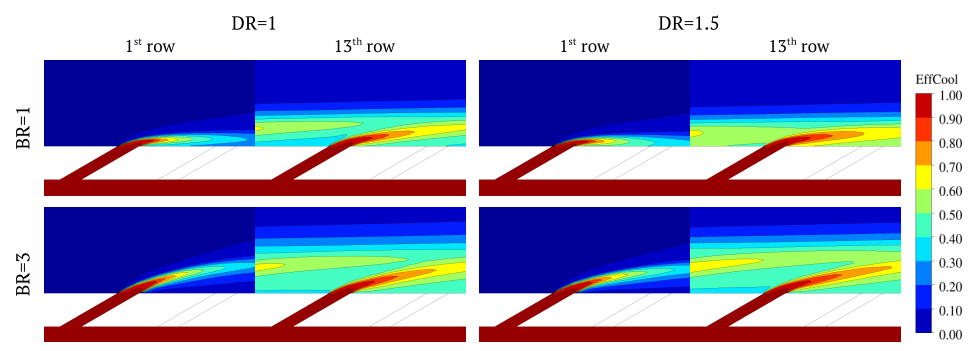

Figure 3.12: Effcool contour plot on symmetry plane. 
showed to be able to correctly predict the experimental trends connected with increasing $B R$ while it highlights a certain insensitivity to inlet turbulence especially at high $B R$. Adiabatic effectiveness was estimated under isothermal conditions by means of passive scalar concentration, temperature difference was exploited to generate a $D R=1.7$. The $D R$ showed some impacts only at the low $B R$ where the penetration of the jet is lower enhancing effectiveness from first to last row of holes.

A comprehensive analysis has been carried out in order to investigate the adiabatic effectiveness performance of a multi-perforated planar plate representative of effusion cooled combustor liners, both from an experimental and a numerical point of view. The selected test case represents a severe benchmark for the numerical models that have to cope with complex turbulent flow field and mixing phenomena due to the multiple coolant injections. Adiabatic effectiveness tests were carried out using Pressure Sensitive Paint Technique, at high turbulence conditions, blowing ratio values ranging from 1 to 3 and density ratio values from 1 to 1.5. Numerical simulations have been conducted exploiting an algebraic anisotropic correction to $k-\omega$ SST turbulence model in order to overcome the well known jet penetration over-prediction and lateral spreading underestimation of classical RANS turbulence modelling.

Detailed measurements of film effectiveness pointed out the effect of the different flow conditions. The higher film wall protection is achieved at low blowing ratio in the first part of the plate (up to $x / S_{x}<6$ ) due to jets lift-off, whereas opposite behaviour is reported for the final part where the superposition effect prevails. Regarding the role of density ratio, this parameter influences the film covering only in the mixing regime, while its effect in the penetration regime can be neglected. Numerical predictions showed to be able to correctly predict the experimental trends connected with increasing $B R$ and $D R$ values. In particular, the effects of Density Ratio variations are captured, returning the opposing effects varying the Blowing Ratio showed by experiments. An underestimation of jets penetration due to RANS modelling has been noticed at every condition, whose effect is however very dependent: a good agreement has 
been achieved in the full penetration regime, whereas at low $B R$ a slight misprediction of the film cooling regime leads to a huge over-prediction in terms of adiabatic effectiveness.

\subsection{Tubular effusion cooled combustor}

\subsubsection{Introduction}

Lean combustion burners are characterized by an internal swirling flow, generating the typical vortex breakdown, which strongly affects the near wall flow both along the liner and on the dome. Thus a reliable estimate of liner thermal protection achieved by means of effusion cooling cannot leave out of consideration the presence of a complex 3D flow field interacting with the coolant jets [58].

The aim of this section is hence to shows the impact of a realistic gas side flow field on cooling effectiveness of a conventional effusion array, already studied in flat plate configuration with uniform crossflow in the previous section. Considered geometry consists of a tubular combustion chamber equipped with a PERM (Partially Evaporating and Rapid Mixing) injector 26] composed by a coaxial double swirler premixer carrying most of combustor air and a nested pilot fuel injector located in the proximity of the combustor axis. The analysis is conducted by means of RANS numerical simulations with a special near wall treatment to better replicate turbulence anisotropy effects of augmenting the jet lateral spreading with respect to standard isotropic formulations [89. The effect of perforation angle is evaluated performing a comparison between slanted and orthogonal holes. Inclination angles were chosen as representative of typical effusion cooling perforations $\left(30^{\circ}\right)$ and of acoustically improved arrays of holes $\left(90^{\circ}\right)$ 85. The cooling system is equipped with a starter 2D slot; two different sets of simulations with activated and deactivated slot cooling were performed to assess its interaction with the main flow as well. All computations are performed under adiabatic conditions and the film protection is estimated through the liner adiabatic effectiveness. 


\subsubsection{Investigated test case}

The investigated test article consists of an effusion cooled tubular combustor as sketched in Figure 3.13 The main chamber is equipped with an injection system, characterized by a double radial swirler whose actual geometry was simplified and made axisymmetric, providing the tangential momentum required to generate a vortex breakdown and hence a swirl stabilized combustion. The liner is cooled by means of a 22 rows effusion cooling perforation, with an axial spacing $S_{a x i} / D=9.15$ and a lateral spacing $S_{t a n} / D=7.37$. Two different inclination angle values have been investigated: $30^{\circ}(L / D=6.25)$ and $90^{\circ}(L / D=3.13)$. The first value $\left(30^{\circ}\right)$ was selected as it represents a standard choice in film cooling applications: lower angles are expected to offer higher overall effectiveness but they are usually not implemented due to elevated manufacturing costs. The second value $\left(90^{\circ}\right)$ was considered as it represents the best choice in terms of acoustic damping capabilities with expected adiabatic effectiveness levels comparable to slanted configuration with high blowing ratio in flat plate case (see Andreini et al. [85]).

The considered liner cooling scheme also employs a 2D-slot, characterized by a relative height $H / D=5$, which have been activated and deactivated to test its interactions with the cold gas injected through the effusion holes and the swirl jet.

The coolant feeding is characterized by a plenum chamber. Even though such a configuration is not fully representative of a combustor engine, it has been decided to investigate this kind of coolant feeding system since it allows uniform conditions upstream of the perforation, hence permitting to assess only the effects due to the variations in the mainstream flow field.

The combustion chamber geometry is periodic in the tangential direction, thus assuming steady state it is possible to take advantage of the rotational periodicity of the flow simulating a single pitch of the liner perforation $\left(\approx 4.09^{\circ}\right)$. This simplification is essential to discretize the perforation with an adequate number of elements maintaining the total computational mesh size below $20 \cdot 10^{6}$ elements. Such assumption is 


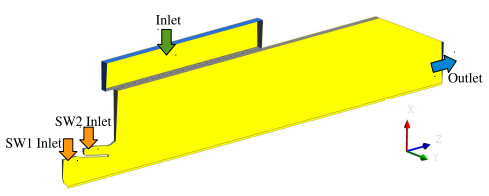

(a) No slot geometry.

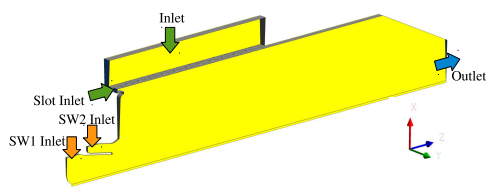

(b) Slot geometry.

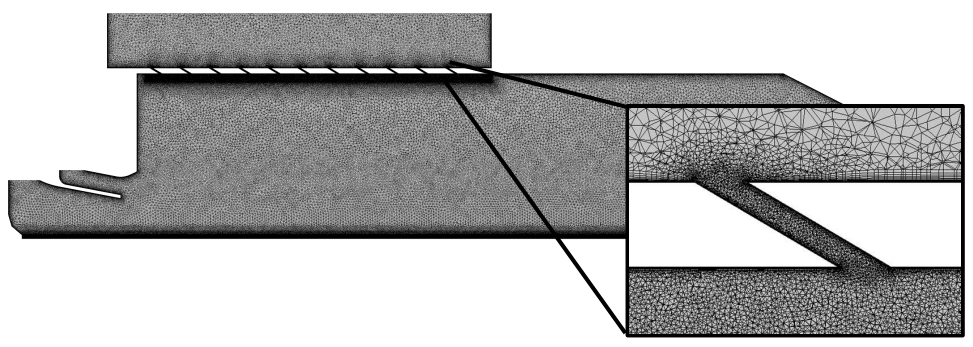

(c) Computational grid.

Figure 3.13: Test case geometries and computational grid.

allowed due to the almost uniform flow field generated by the injector which is equipped with a large number of foils: for this reason the injection system is not included in this computation. The test matrix of studied geometries is reported in Table 3.3

Table 3.3: Test matrix.

\begin{tabular}{c|c|c|c}
\hline \hline Geom & $\alpha\left[^{\circ}\right]$ & $D R[-]$ & $m_{\text {slot }} / m_{\text {main }}$ \\
\hline \multirow{2}{*}{ G2 } & \multirow{2}{*}{30} & 1 & $0 \%$ \\
& & 2.5 & $0 \% 3 \% 6 \%$ \\
\hline \multirow{2}{*}{ G7 } & \multirow{2}{*}{90} & 1 & $0 \%$ \\
& & 2.5 & $0 \% 6 \%$ \\
\hline \hline
\end{tabular}




\subsubsection{Numerical setup}

\subsubsection{Numerical settings}

Calculations have been carried out following a 3D CFD RANS approach referring to the Navier-Stokes solver ANSYS ${ }^{\circledR}$ CFX v14. Regarding fluid properties, a compressible ideal gas was considered with the solution of energy conservation in terms of total energy including viscous heating effects. An High Resolution scheme has been used to discretize momentum and scalar equations.

Turbulence was modelled by means of the $k-\omega$ SST turbulence model with an algebraic anisotropic correction suited to overcome typical RANS modelling failures for film cooling flows as described up ahead. Employed near wall treatment uses an automatic blending between Wall Function and Wall Integration approach on the basis of $y^{+}$value. For the cases presented in this work where $\max \left(y^{+}\right)<1$, the Low Reynolds formulation is always recovered.

The solution convergence has been assessed by monitoring that rms residuals fell below a prescribed value of $1 e-6$ and verifying that the averaged value of $\eta_{a d}$ on the plate reached a steady-state condition.

\subsubsection{Boundary conditions}

The boundary conditions at the swirlers have been assigned in terms of mass flow rate and velocity direction providing equivalent conditions as obtained with a pressure drop of $3.5 \%$ across the injector. Total temperature and turbulence quantities are fixed at the inlet as well, while a static pressure value of $101325 \mathrm{~Pa}$ is imposed on the outlet. Concerning the effusion coolant, the pressure drop across the liner was maintained at $3.5 \%$. The cases with slot cooling benefit of an additional coolant flow rate established around $6 \%$ of the air flow through the injector, which in the experience of the authors is representative of annular lean combustors. Total temperature of both coolant streams was fixed to $298.15 \mathrm{~K}$, whereas the value at the swirler inlet, since different conditions of density ratio $D R$ have been investigated, has been varied from $298.15 K(D R=1.0)$ 
to about $723.15 K(D R=2.5)$. Walls have been assumed smooth and adiabatic, with a no slip condition.

In order to assess the coolant distribution and verify separately the effects of slot and effusion cooling, two transport equations for additional passive scalars were solved: EffCool and SlotCool, representing effusion and slot coolant concentration respectively. The values imposed at the inlets are reported in Table 3.4 Both turbulent Prandtl and Schmidt numbers have been fixed to 0.9 , so as to obtain a Lewis number equal to 1 , with the aim of fully respecting the mass-energy transfer analogy.

Table 3.4: Boundary conditions for additional transport equations.

\begin{tabular}{cccc}
\hline \hline & Swirler Inlet & Effusion Inlet & Slot Inlet \\
\hline EffCool & 0 & 1 & 0 \\
SlotCool & 0 & 0 & 1 \\
\hline \hline
\end{tabular}

\subsubsection{Grid generation}

ANSYS $^{\circledR} \quad$ ICEM-CFD has been used to generate hybrid computational grids (tetrahedral with 15 layers of prisms close to the wall). The axisymmetric condition (see Figure 3.13 has been exploited to reduce the computational effort. The meshing criteria adopted are derived from the mesh sensitivity analysis carried out in [90, which led to about 20 million of elements (Figure 3.13c). As it is possible to notice, following the cited criteria the grid has been finely clustered nearby the plate and within the holes in order to capture the flow physics.

\subsubsection{Results}

All the results presented have been obtained using the anisotropic correction of the $k-\omega$ SST turbulence model. The standard isotropic formulation was also tested in this work reporting much smaller discrepancies among these two approaches than on flat effusion plates [90, since in this case the mixing is strongly influenced by the interaction with the internal 
flow field. Therefore, in order to make a more coherent dissertation, the results achieved with the standard model have been omitted.

The flow field generated by the injector in absence of liner cooling schemes is characterized by an internal recirculation zone generated by the swirled flow and a counter rotating vortex in the corner of the combustor, as reported in [27]. Corner recirculation extends to a position corresponding to the $10^{\text {th }}$ row of effusion holes where the stagnation point of the principal flow occurs while the central bubble occupies a large zone of the combustor ending downstream last row of the effusion array. The outer swirler presents a higher velocity at the inlet of the chamber compared to the inner one; this is mainly due to higher tangential velocity components rather than stronger axial momentum.

\subsubsection{Perforation angle effects}
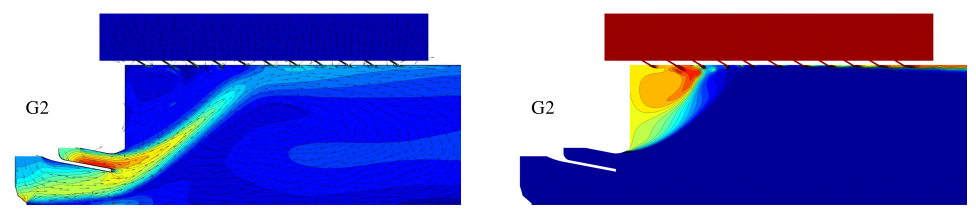

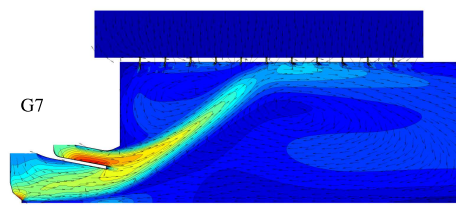

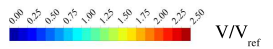

(a) $V / V_{\text {ref }}$ distribution on meridional plane.

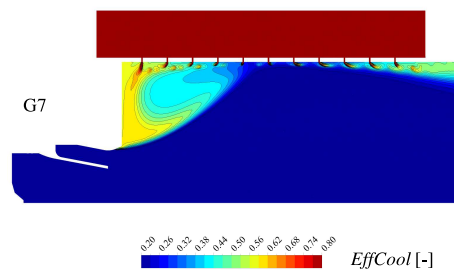

(b) EffCool distribution on meridional plane.

Figure 3.14: Influence of perforation angle $(D R=1)$.

The flow field obtained at unitary density ratio is reported in Figure $3.14 \mathrm{a}$ in terms of $V / V_{\text {ref }}$, where a comparison between the different perforations is depicted: contours are reported on a meridional plane 
cutting in half the odd rows. As it was reasonable to expect, minor discrepancies can be noticed concerning the main recirculation region. Nevertheless, it is certainly more interesting to focus on the corner of the flame tube, for which in case of the orthogonal perforation (G7), an anti-clockwise vortex occurs. This phenomenon is due to the swirl flow and does not seem affected by the injection of coolant in a direction normal to the wall. On the other hand, when the slanted perforation is considered (G2) the interaction with the injected coolant leads to a momentum exchange that induces an inversion of the rotation direction characterized by a strong shear layer with the main recirculation region.

This different flow field thereby affects the distribution of coolant in the first part of the liner: as can be seen in Figure $3.14 \mathrm{~b}$ the anti-clockwise recirculation vortex (in G7 case) blows the coolant coming out from the first rows against the heat shield, wiping it out from the liner wall (in conjunction with the perforation angle effect), reducing its protection ability. The two vortices generated by slanted perforation instead interact in such a way to generate positive axial velocity in the near wall region that pushes coolant jets towards the wall permitting film accumulation thus leading to a higher protection of the liner. These considerations are confirmed also by the laterally averaged values of adiabatic effectiveness presented in Figure 3.15 slanted perforation presents much higher film protection for the first and the last sections of the liner wall.

From Figure 3.15, it is also possible to observe two $\eta_{\text {ad }}$ peaks in G2 case: the first peak (related to the $4^{\text {th }}$ row) is caused by the interaction of the clockwise and the anti-clockwise vortices bringing to a stagnation point; the second peak (related to the $8^{\text {th }}$ row) correspond to the main stagnation region caused by the swirl flow.

In the liner final section, it is possible to find out the effect of the different perforation angle on coolant behaviour: as expected, normalto-wall holes lead to a complete detachment of the jets from the liner wall, with a consequent penetration regime. On the other hand, slanted rows guarantee wall attached jets, see Figure 3.14b, providing high film protection. 


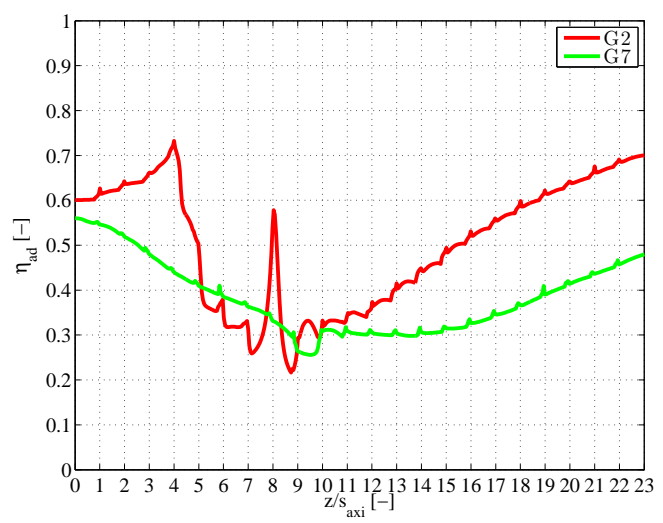

Figure 3.15: Laterally averaged adiabatic effectiveness profile on liner wall: perforation angle influence $(D R=1)$.

\subsubsection{Density Ratio effects}

Density ratio represents a key parameter for the characterization of film cooling performance. Experimental measurements are usually carried out with small density ratio such as $D R \approx 1$, whereas the typical coolant to mainstream values for engine conditions is $D R \approx 2.5$. Nevertheless, it has been proved in literature that density ratio plays a minor role in the resulting film effectiveness if compared to other parameters such as momentum flux ratio 4, 91. In this case however, as the global $B R$ remains the same, $D R$ and $M R$ are directly linked so that $M R \propto 1 / D R$.

It is worth to remember that in the context of this activity, density ratio variation is achieved increasing the temperature of the hot gas. The effect of this parameter is shown in Figure 3.16, where the adiabatic effectiveness distribution on the liner wall is depicted.

For what concerns the slanted perforation (G2), the density ratio increase does not involve significant alterations on the stagnation point location which is only shifted only one row downstream, whereas in the last part of the liner several differences can be noticed in the pattern 


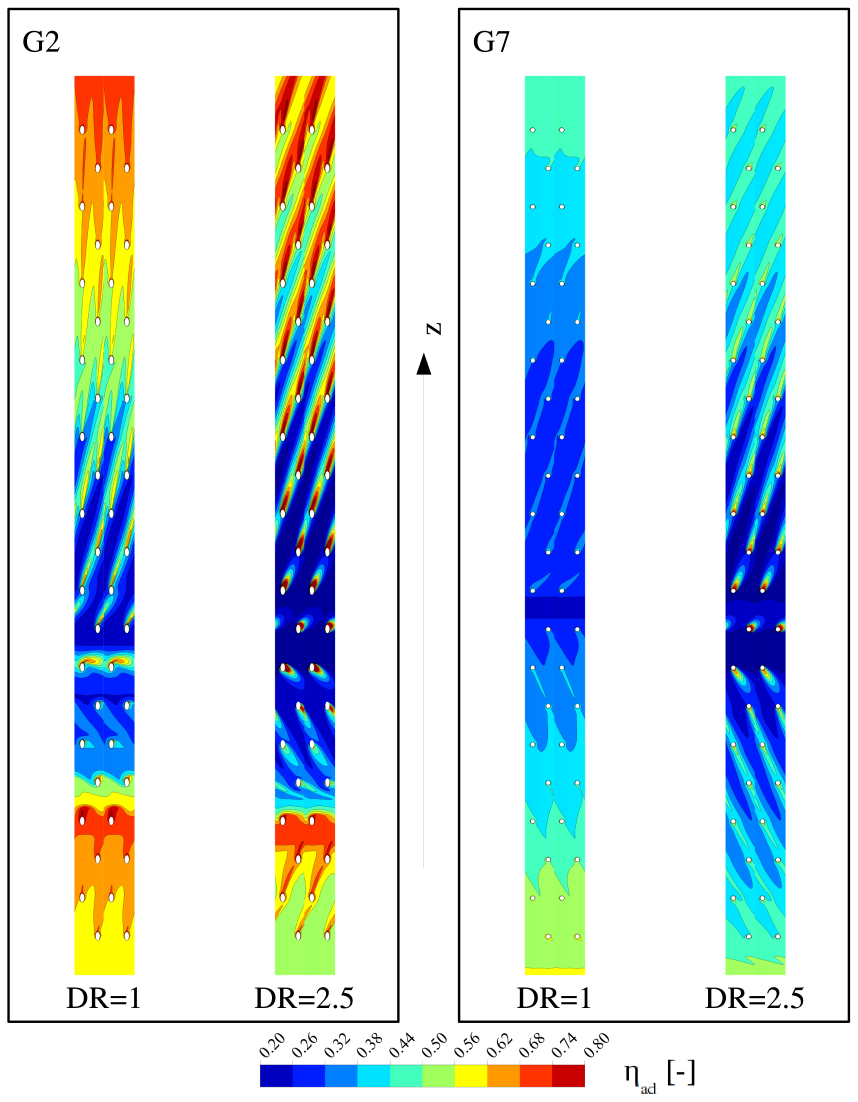

Figure 3.16: Density ratio effect on adiabatic effectiveness distribution on liner wall.

of coolant protection. In case of unitary density ratio, jet trajectory seems characterized by a mainly axial direction, while for $D R=2.5$ the tangential component of the hot gas is maintained up to the end on the 
plate. This phenomenon can be ascribed to the momentum exchange between coolant and mainstream that, at lower $D R$, is dominated by the jets. On the contrary, when the higher $D R$ is considered, the higher momentum of the mainstream becomes predominant compared to the discrete jets and is able to provide swirled velocity in the near wall region as well. Furthermore, another effect is represented by a greater film effectiveness along the wakes, which can be ascribed to the reduction of momentum ratio responsible for a lower penetration of the jets. Initial part of the liner is not favoured by the enhanced $D R$; in the absence of film starter, adiabatic effectiveness on the first rows is dominated by coolant convected by the corner recirculation. The higher jet penetration which characterize $D R=1$ is hence beneficial.

Looking at Figure 3.17a it is possible to confirm how the change in $D R$ value brings to a sensible effect in the first part of the liner, and how after the stagnation region the two $\eta_{a d}$ profiles are closer.

Despite the different corner vortex dynamics, which generates opposite oriented jet wakes, the effects of $D R$ on the first rows are equivalent also in case of orthogonal perforation (G7). Stagnation point moves downstream of approximately a row confirming that main flow generated by the injector is quite independent from the near wall field. In terms of wake direction the last part of the liner looks almost insensitive to the variation of the density ratio because in both conditions full penetration regime is achieved. Anyhow the lower momentum ratio of $D R=2.5$ simulation generates an increased wake protection in the wakes. This phenomenon provides a sensible increase in laterally averaged effectiveness, as reported in Figure $3.17 \mathrm{~b}$ but is completely balanced at the end of effusion plate.

\subsubsection{Comparison with flat plate configuration}

In order to assess the relevance of the current study for design purposes, it is of interest to compare obtained results with those related to uniform cross-flow conditions. In particular it is convenient to exploit the results available from a previous analysis focusing on exactly the same 


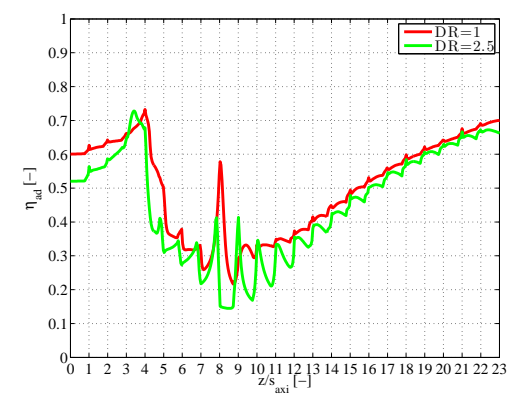

(a) G2 geometry.

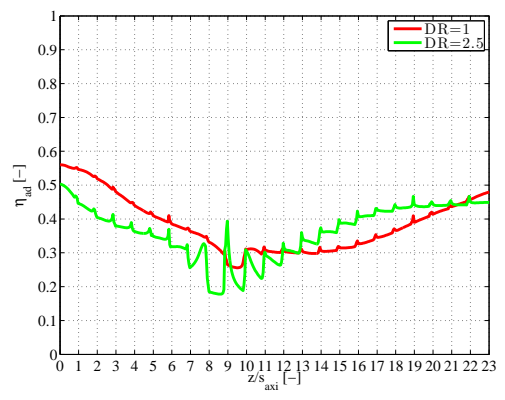

(b) G7 geometry.

Figure 3.17: Laterally averaged adiabatic effectiveness profile on liner wall: DR influence.

perforations but applied to a flat plate [89. In fact, being based on the same computational strategy, validated in that case against experimental values, it is possible to minimize modelling discrepancies even though such analysis considered 18 rows of holes only. To further minimize the effects of the different set-up, local blowing ratio of tubular case are computed to choose the most representative flat plate flow condition to compare with. As reported in Figure $3.18, B R$ is quite variable along the liner assuming highest values close to the combustor dome where the main flow is inhibited by the corner recirculation. The lowest values, limited to around 1 , are instead localized just downstream the main vortex stagnation zone. As the early part of the perforation is affected by main flow oriented in opposite direction respect to the major part of the liner, the blowing ratio of interest among the investigated one $(B R=1,2,3)$ was selected looking at the behaviour for $x / s_{a x i}>10$. As it is proved that the effect of density ratio is negligible for flat plate configuration in case penetration regime is established, the comparison was conducted at unitary density ratio choosing for both geometries $B R=2$ condition. This choice is also supported by the average blowing ratio reported by experiments with a pressure drop across the plate of $3.5 \%$ which is comprised between 2 and 3 for both G2 and G7. 


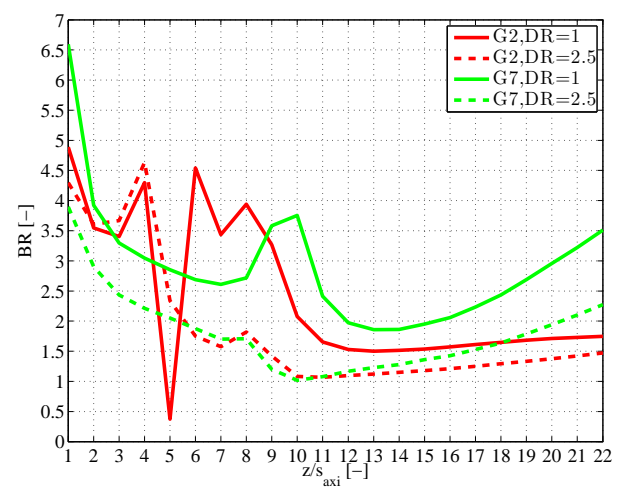

Figure 3.18: Local blowing ratio along the liner: influence of DR and perforation.

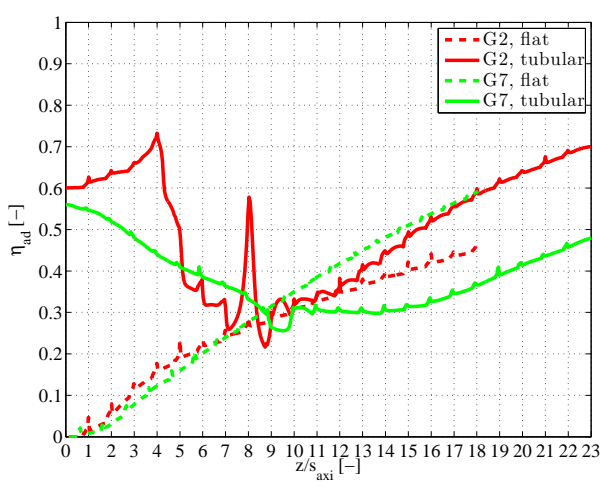

Figure 3.19: Laterally averaged adiabatic effectiveness profile on liner wall: comparison between flat plate and tubular combustor $(D R=1$, $B R=2$ )

As reported in Figure 3.19 it is possible to notice how the film protection is strongly altered by the swirling flow for both the perforation angles. In particular initial part of the effusion presents much higher film protection for the tubular combustor compared to the flat plate case 
where initial level is obviously null. However also the final part of the plate is affected by the main flow field resulting in an inverted behaviour for the two hole angles. For flat plate configuration in fact, G2 and G7 showed comparable levels of coverage with G7 being slightly more protective, opposite trend is instead verified in the current analysis as already discussed in a previous section.

Concerning the density ratio effects, flat plate analysis (which was limited to $D R=1.7$ in [90] and extended to $D R=2.5$ in [91]) highlighted almost no effect on adiabatic effectiveness. Although certainly more relevant, the changes in laterally averaged effectiveness are limited for the tubular configuration as well permitting to extend these findings also to high density ratio conditions.

\subsubsection{Slot effects}

The effects on the liner coolant distribution due to the introduction of the slot cooling are reported in Figure 3.20 This case is particularly interesting due to the intrinsic limits of the effusion cooling, which is characterized by a poor coolant protection of the first rows, usually compensated by means of a slotted film starter. It is therefore relevant to evaluate the contribution of the slot cooling on the global film effectiveness, especially in the context of the use of different drilling typologies.

Regarding the G2 geometry, slot cooling provides a very high and uniform protection on the first rows, whereas the effect decreases suddenly between the fourth and the fifth rows. This phenomenon can be ascribed to the flow field nearby the corner region, as highlighted in the previous sections. The mixing between the coolant coming from the slot and the mainstream, depicted in Figure 3.21, produces a mild increase of $\eta_{a d}$ up to the $9^{t h}$ row, before becoming negligible downstream of the stagnation point.

When the G7 geometry is considered, it is evident how slot fails in providing any protection on the liner. The different flow field in the outer recirculation zone, characterized by an counter-clockwise vortex, prevents the coolant from remaining attached to the wall, thus being removed 


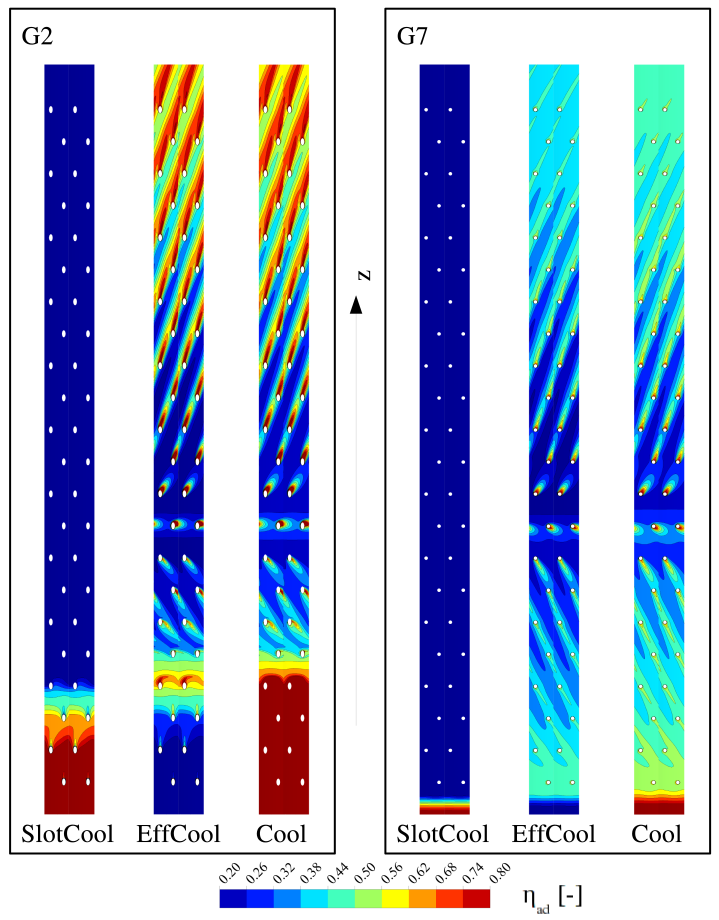

Figure 3.20: Slot coolant effect on adiabatic effectiveness distribution on liner wall.

and dragged away, as shown in Figure 3.21 As in the case of G2, the coolant, even though does not contribute directly to the protection of the liner, is affected by a phenomenon of mixing which extends in the whole corner vortex, providing a slight contribution to the liner cooling up to the end of the plate. As it is possible to notice looking at Figure $3.22 \mathrm{~b}$. where laterally averaged profiles of the three tracers are reported, the SlotCool term is transported up to the end of the liner wall. Moreover, it is possible to see how the effect of the slot cooling is relevant only up 


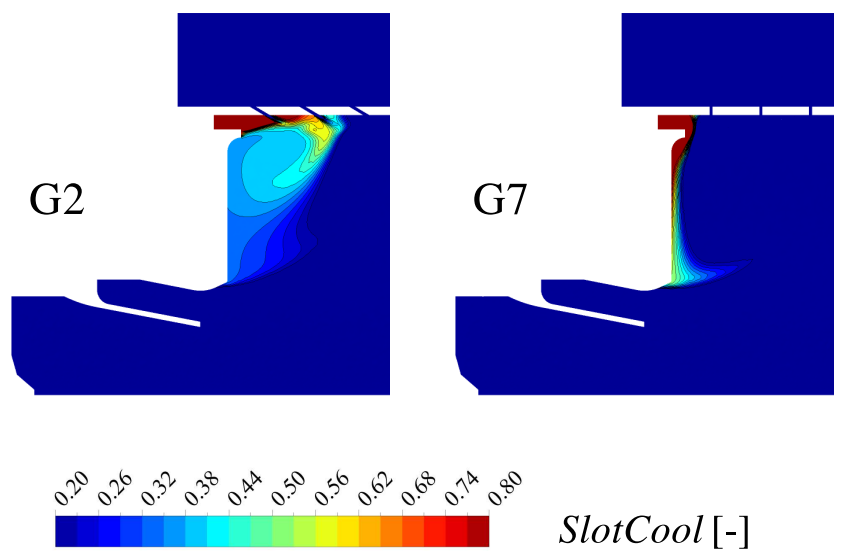

Figure 3.21: Slot coolant distribution on meridional plane.

to the stagnation point, since the characteristics of the flow field lead to the entrapment and recirculation of its stream in the corner region. Furthermore, as described above, only the slanted perforation succeeds in guaranteeing a relevant contribution to the film effectiveness in proximity of the first rows (Figure $3.22 \mathrm{~b}$.

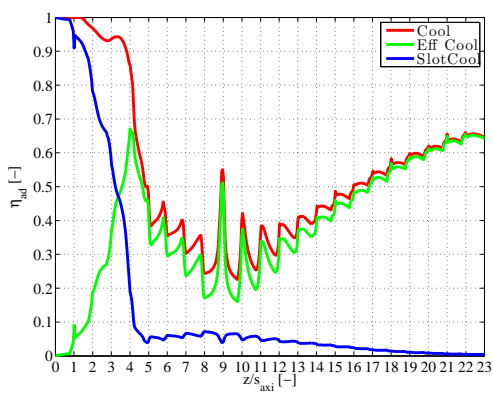

(a) G2 geometry.

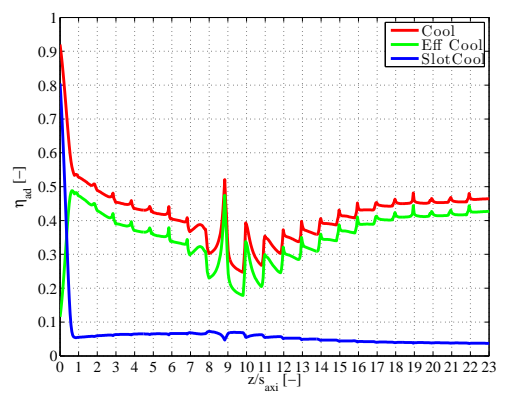

(b) G7 geometry.

Figure 3.22: Laterally averaged adiabatic effectiveness profile on liner wall: slot cooling injection influence. 
In a design perspective, it is of interest to evaluate the impact of the slot cooling mass flow rate on the distribution of adiabatic effectiveness on the liner, in particular it may be convenient to investigate efficiency of the film starter also at reduced mass flow rates. As for G7 even $6 \%$ of global mass flow is not sufficient to maintain the slot jet attached to liner, the test with a reduction of $50 \%$ was carried out for G2 only.

The results are reported in Figure 3.23, where a comparison between the contributions of slot and effusion cooling at the different operating conditions have been depicted. As it was possible to expect, the main discrepancy is restricted to the first rows of holes, where the lower momentum leads to a lower penetration of the flow. The variation of the mass flow rate does not modify the global structure of the three recirculation zones, therefore the contribution of the slot cooling in both conditions is relevant just up to the $4^{\text {th }}$ row, after which only the mixing with the hot gas ensures a minimal effect on the protection of the liner. Nevertheless, it is interesting to notice that the reduction of the role played by slot cooling is actually associated to a greater presence of coolant coming from the effusion cooling holes, which is clearly beneficial from the point of view of the global adiabatic effectiveness, since it contributes to compensate the effects due to the lower slot cooling mass flow rate.

In conclusion, considering that $\mathrm{m}_{\text {ef fusion }}$ is roughly unchanged in the two simulations, a reduction of $50 \%$ in $\mathrm{m}_{\text {slot }}$ involves, for the configuration of combustor under investigation, a reduction of $10 \%$ in the overall cooling mass flow rate. In these conditions the distribution of adiabatic effectiveness is mostly not affected, with the exception of the first part of the liner, since up to the $3^{\text {rd }}$ row $\eta_{a d}$ is about $10 \%$ less than the reference case.

\subsubsection{Remarks}

The film effectiveness of effusion cooling systems is deeply influenced by the interaction with the mainstream generated by the injection systems, therefore the investigation on flat plates is not sufficient for an accurate assessment of the cooling performance of effusion cooled liners. 


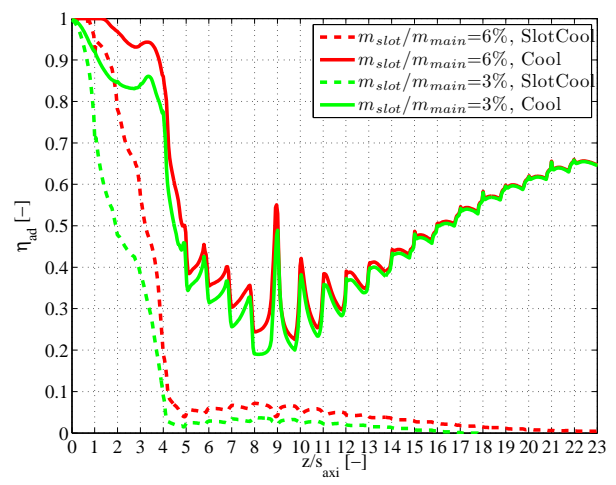

Figure 3.23: Laterally averaged adiabatic effectiveness profile on liner wall: slot cooling mass flow rate influence.

A numerical activity has been carried out in order to investigate the adiabatic effectiveness of typical effusion array in presence of a realistic flow field generated within a tubular combustor. The investigation has been focused on two different perforations $\left(30^{\circ}\right.$ and $\left.90^{\circ}\right)$ on representative values of density ratio and in conjunction with a slotted film starter.

For what concerns the perforation, it has been possible to highlight a huge interaction between the coolant injected through discrete holes and the mainstream. In particular, the slanted perforation provides a momentum transfer that leads to the inversion of the typical counterclockwise recirculation vortex. This zone is subject to a relevant mixing that contributes to lower the temperature of the gas in the corner region providing a high coolant protection on the first rows, which on simplified configurations are usually characterized by poor coolant superposition and hence low values of adiabatic effectiveness.

For this reason, usually the combustors are equipped with film starter devices. When the slot cooling is introduced, it has been highlighted that stream momentum is not sufficient to modify the flow field, thus only in the case of the slanted perforation it succeeds in providing relevant protection on the first rows of the liner, whereas in the case of the 
orthogonal perforation it is dragged away, contributing only through the mixing with the hot gas. Overall, slanted perforation better performs in terms of liner coverage opposite to what revealed by flat plate analysis, which in penetration regime showed higher levels of effectiveness for the normal holes.

For what concerns the design of liners for lean combustors, this study has pointed out the importance of a detailed investigation of the coupling among effusion cooling jets, starter film and realistic swirler flow field. Obtained results have pointed out the possibility to define optimized effusion cooling perforations by selecting local inclination angles so as to maximize adiabatic effectiveness with respect to local cross-flow. As discussed in the introduction inclination angle can also be locally modified so as to maximize acoustic damping. Nevertheless obtained results cannot be directly considered as a final design input as the selection of an optimized configuration should be carried out also taking into account the heat transfer inside cooling holes, greatly affected by their length to diameter ratio.

\subsection{Annular slot cooled combustor}

\subsubsection{Introduction}

In addition to the above mentioned limitations inherent to RANS modelling, the simulation of swirling flows presents further issues due to the intrinsic unsteadiness of the flow field. For the sake of clarity it is possible to refer to Fig. 3.24 where a comparison among the measured and predicted cold flow fields on a linear array of three swirlers at nonreacting conditions is reported. As it is possible to notice, the RANS of the single sector, with an imposed translational periodicity, reproduces an non-physical behaviour, characterized by the interaction across the sector frontiers that leads to a dome-attached swirling flow. It is interesting to highlight that the simulation of the entire three sector rig returns a completely different result, with a periodic flow pattern featuring the alternation of compact inner recirculation zones (IRZ) and dome-attached 
swirling flows.

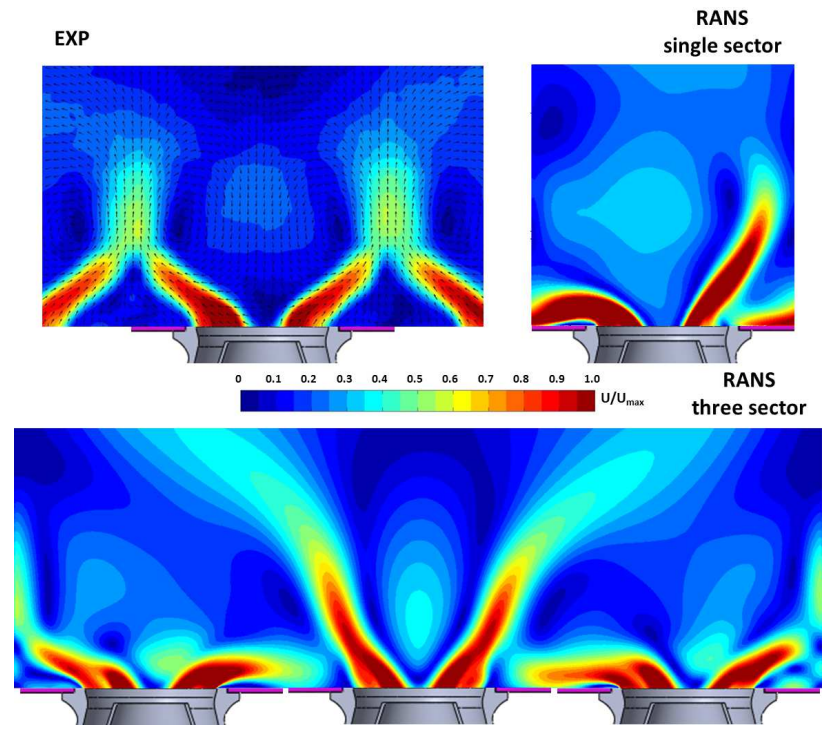

Figure 3.24: Measured and predicted flow field on the median plane

Such phenomenon, yet not confirmed by the PIV measurements, was investigated experimentally by Kao et al. [92, who studied a linearlyarranged 5-swirler array at non-reactive conditions. The authors investigated the impact of inter-swirler spacing, lateral walls distance and dome recession on the flow field, pointing out their effect in switching the swirling flow behaviour to a wall-attached configuration, generating air entrainment from the adjacent injector (see Fig. 3.25).

Following such results, it appears evident that small errors in predicting the structures of the swirling flows can deeply affect the resulting flow field, potentially leading the RANS models to switch from an unstable condition to the dome-attached configuration. For this reason, the CFD investigation of swirling flow of particular geometries (e.g. unconfined flows) and at cold flow operating conditions (it is worth remembering 


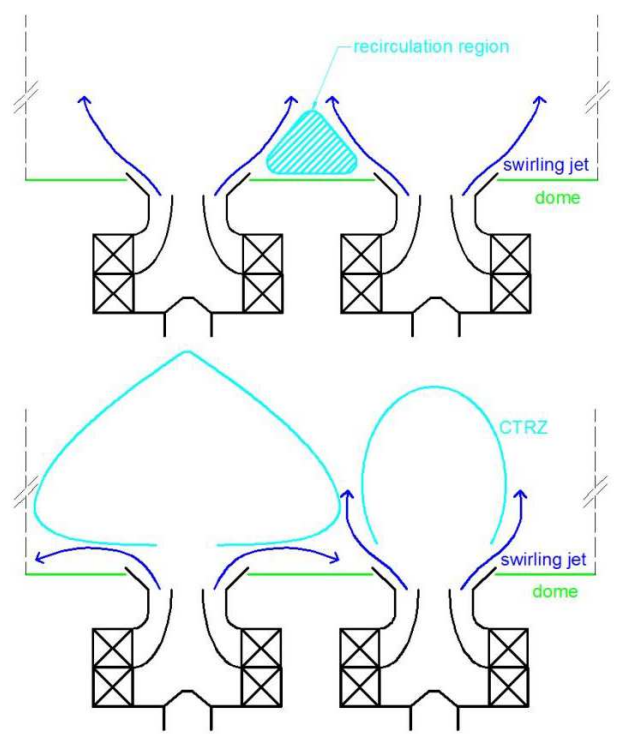

Figure 3.25: Classical flow pattern of an array of swirles (top) and dome-attached swirling flow (bottom) [92]

in fact that instability related to the presence of the PVC is drastically suppressed by heat release in reacting flows [93]) may results infeasible with standard RANS approach.

The aim of this work is to investigate the heat transfer augmentation on combustion liners under the effect of a realistic gas side flow field. The considered geometry consists of the three-sector planar rig developed in the context of the EU project LEMCOTEC [60]. This test case can be considered of particular interest, being characterized by a strong interaction between adjacent injectors, due to the absence of any lateral confinement of the swirled flow, and coolant injection through a slot embedded in the combustor dome.

Simulations were carried out imposing realistic combustor operating conditions, especially in terms of reduced mass flow rate and pressure drop 
across the swirlers. The analysis was conducted by means of unsteady simulations carried out with hybrid RANS-LES turbulence models. All the computations were performed fixing a constant wall heat flux conditions on the liner, so as to estimate the Nusselt distribution. To the authors' knowledge it represents the first attempt to perform an heat transfer investigation on this kind of configuration representative of an aero-engine annular combustor.

\subsubsection{Investigated test case}

The experimental arrangement, depicted in Fig. 3.26 consists of a non-reactive three sector planar rig installed in an open loop wind tunnel; the rig is characterized by three swirlers and a simple cooling scheme made up of a slot injection, reproducing the exhaust dome cooling mass flow of a real engine configuration. The test rig allows the control of two separate flows: the mainstream and the slot cooling flows. The most important features concerning the test rig and the experimental techniques are reported below, for further details please refer to Andreini et al. 60.

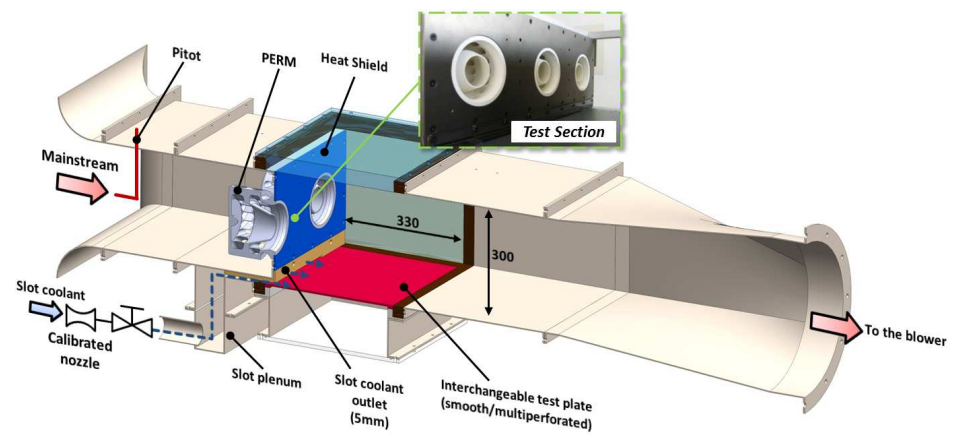

Figure 3.26: Experimental apparatus

Before entering into the test section, the mainflow is swirled by a set of injectors fixed at the dome in a planar arrangement; three swirlers are installed to reproduce the periodicity of an annular combustor chamber 
and to reduce side-wall effects on the central investigation area. The scaled injectors reproduce the real geometry of an AVIO Aero PERM (Partially Evaporated and Rapid Mixing) injector [27], which is developed for Ultra Low $\mathrm{NO}_{x}$ (ULN) combustor core technology and it is addressed to achieve partial evaporation inside the inner duct and a rapid mixing within the combustor, optimising the flame position and the stability of the combustion process. It is characterized by two radial co-rotating primary and secondary swirlers used to assign angular momentum on the two distinct inlet air flow; before entering in the combustion region, the swirled flows are accelerated in the inner and the outer nozzles thanks to a conical internal body.

The test section reproduces an aero-engine lean combustor chamber scaled up with respect to the actual configuration; the smooth test plate, placed on the bottom wall of the test section, represents the inner liner of the combustion chamber and it is made of a low conductive material. The slot exit is located below the three swirlers; it has a constant height of $6 \mathrm{~mm}$ and it extends between the axis of the two lateral swirlers. The slot system, used to generate film cooling in the very first part of the test plate, is fed by cooling air using a plenum chamber connected directly upstream the slot exit and provided with screens and flow straighteners.

Despite the simple configuration of the cooling system that will be tested in this work, the rig is designed to investigate also the complete cooling scheme of a typical combustor liner equipped with a PERM injector: together with the slot, it includes an effusion cooling system, which can be reproduced mounting a multi-perforated plate in place of the smooth one. The effects of the mutual interaction between this arrangement and the flow field generated by the injectors are not investigated in the context of this paper.

\subsubsection{PIV measurements}

Thanks to the large optical accesses, it was possible to introduce the light sheet at different locations in the test rig: as sketched in Figure 3.27. measurements were carried out either in the center plane, positioned 
orthogonally to the liner wall and crossing the axis of the central swirler, and in the median plane, parallel to the wall and crossing each centre of the three injectors.

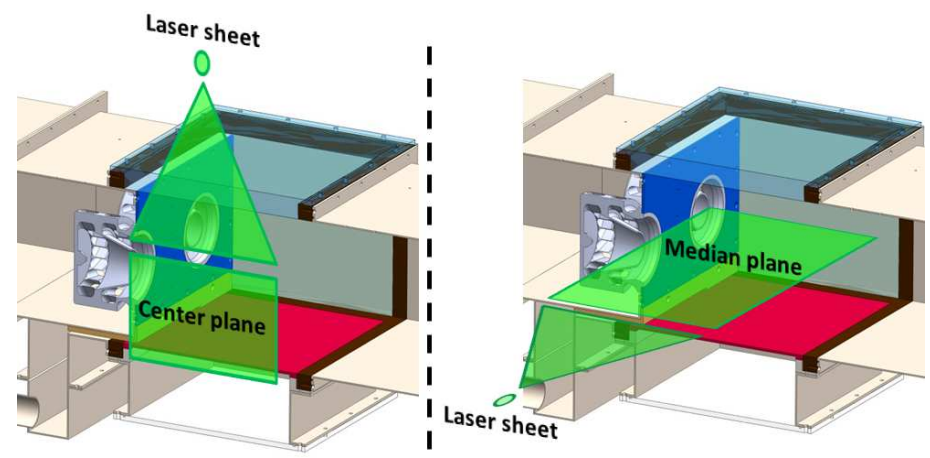

Figure 3.27: Position of the PIV measurements planes

\subsubsection{HTC measurements}

Heat transfer coefficients were determined by a steady-state technique, using TLC paint to measure the wall temperature of the heated surface of the test plate; both mainstream and cooling flows were at room temperature.

The heat transfer coefficient is defined as follow:

$$
H T C=\frac{q_{c o n v}}{T_{w}-T_{a w}}
$$

where the $T_{a w}$ represents the surface temperature of a perfectly insulated wall. As the mainstream Mach number was low, recovery effects can be neglected and therefore the mainstream temperature, measured with thermocouples upstream the three injectors, was used as adiabatic wall temperature. $T_{w}$ is the measured wall temperature recorded during the experiments by means of TLC; $q_{\text {conv }}$ represents the heat flux exchanged by convection between the test plate and the main flow. A small fraction 
of the heat generated by Joule effect (about 3\%) is not exchanged by convection and represents the heat loss due to conduction through the PVC plate and the following convection on its back side. Heat loss was taken into account during the post-processing of the experimental data: to obtain the distribution of $q_{c o n v}$ on the surface of the test plate, for each pixel of the recorded image a simple 1D approach (conduction through the test plate and natural convection) was employed to estimate the overall losses and then subtracted to the total surface heat flux.

The uncertainty of HTC measurements was evaluated following on the method proposed by Kline and McClintock 94 and on a confidence level of $95 \%$. It was estimated to be $8 \%$, taking into account the uncertainties in calibration and temperature measurements.

\subsubsection{Test conditions}

The experimental campaign was performed imposing several fluid dynamics conditions ranging among typical engine values. The main investigation parameter of the mainflow is the pressure drop across the injection system defined as follow:

$$
\frac{\Delta P}{P}=\frac{P_{u p}-P_{d w}}{P_{u p}}
$$

where $P_{u p}$ is the average wall static pressure measured in the inlet duct directly upstream the heat shield, while $P_{d w}$ is the wall static pressure measured downstream the test section. Concerning PIV measurements, tests were performed only at a single value of $\Delta P / P=3.5 \%$, therefore only this condition has been investigated in the context of this paper. Preliminary numerical calculations, in fact, showed that reasonable variations of pressure drop, as imposed in the experimental campaign, do not deeply affect the main characteristics of flow field: varying this parameter, the major flow structures remain unchanged and the main modifications deal only with the intensity and the inclination of the jet coming from the swirlers.

The Reynolds number of the mainstream is evaluated as: 


$$
R e_{\text {main }}=\frac{m_{\text {main }} \cdot d_{h}}{A_{\text {main }} \cdot \mu_{\text {air }}}
$$

where $d_{h}$ is the hydraulic diameter and $A_{\text {main }}$ is the cross section area of the investigated test section. In the range of the imposed pressure drop across the swirlers $(\Delta P / P=2-5 \%)$, the Reynolds number is between 129000-200000.

Concerning the slot system setup, different values of the cooling mass flow were investigated experimentally to evaluate the effect of coolant injection through the slot $\left(m_{\text {slot }}=0-3 \%\right)$. Nevertheless, only the highest mass flow rate was tested numerically, being representative of the actual conditions employed in combustors.

The heat transfer results are presented in terms of Nusselt number augmentation ratio, which is the measured Nusselt number normalized by means of a reference value obtained from the correlation proposed by Dittus-Boelter [95] for fully developed flow:

$$
N u_{0}=0.023 \cdot \operatorname{Re}_{\text {main }}^{0.8} \cdot \operatorname{Pr}^{0.3}
$$

\subsubsection{Numerical setup}

Calculations have been carried out following a 3D CFD unsteady approach referring to the Navier-Stokes solver ANSYS ${ }^{\circledR}$ CFX v15. Regarding fluid properties, due to the low Mach conditions and the little variations in term of temperature, the fluid has been treated as incompressible and with constant properties. A Bounded Central Difference scheme was used for the spatial discretization of the resolved equations, in conjunction with a second order backward Euler transient scheme.

Turbulence was modelled by means of two different hybrid RANS-LES models: Scale Adaptive Simulation (SAS) and Detached Eddy Simulation (DES), in their formulations made available by the solver, based on the RANS SST turbulence model. Employed near wall treatment uses an automatic blending between Wall Function and Wall Integration approach on the basis of $y^{+}$value. For the cases presented in this work where 
$\max \left(y^{+}\right)<1$, the Low Reynolds formulation is always recovered.

The simulations have been carried out with a time step of $1 \mathrm{e}-5 \mathrm{~s}$, derived from a sensitivity analysis and small enough to provide a Courant number lower than 1 . The simulations were stopped after $0.2 \mathrm{~s}$, a time frame able to consider more than 40 passages through the swirler.

\subsubsection{Boundary conditions}

A sketch of the computational domain is reported in Fig. 3.28. The boundary conditions at the inlet was assigned in terms of mass flow rate and temperature $(300 K)$, whereas an opening condition was applied at the outlet, with the pressure value measured experimentally for the required pressure drop condition. The cases with slot cooling benefit of an additional coolant flow rate, at the same temperature of the main inlet. All the walls were treated as smooth and adiabatic, with a no slip condition, except for the flat plate, to which a constant heat flux condition was applied.

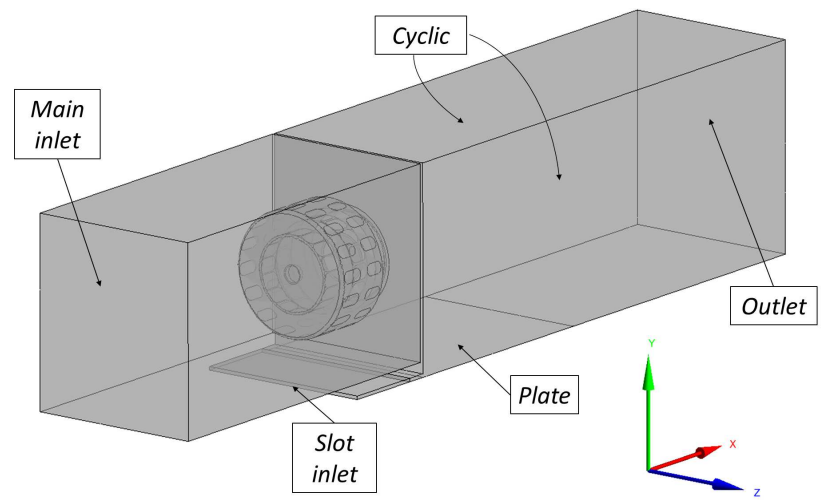

Figure 3.28: Sketch of the computational domain 


\subsubsection{Grid generation}

ANSYS ${ }^{\circledR}$ ICEM-CFD has been used to generate hybrid computational grids (tetrahedral with 20 layers of prisms close to the wall). The meshing criteria adopted are derived from the best practices drawn up by the code developers for Scale Resolved Simulations [96. Nevertheless a mesh sensitivity analysis was carried out to assess the impact on the predicted flow field and Nusselt number distribution. The characteristics of the grids employed for the simulations are reported in Tab. 3.5 (see also Fig. 3.29

\begin{tabular}{lccc}
\hline \hline & Min size $($ Elem $/ D)$ & Elements $\left(10^{6}\right)$ & Nodes $\left(10^{6}\right)$ \\
\hline M1 & 28 & 7.98 & 2.56 \\
M2 & 83 & 20.63 & 5.42 \\
\hline \hline
\end{tabular}

Table 3.5: Main features of the computational grid
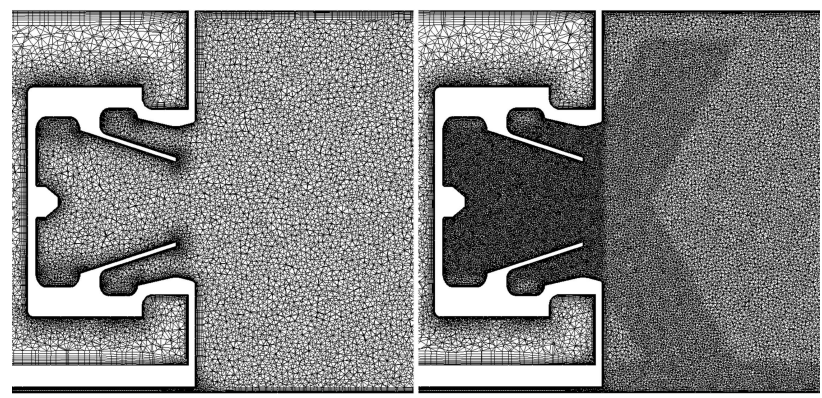

Figure 3.29: Computational grids: Mesh 1 (left) and 2 (right)

\subsubsection{Turbulence modelling - Detached Eddy Simulation}

With the purpose of improving the accuracy of turbulence models in regions characterized by strong unsteadiness and separation, Spalart et al. 97 proposed an hybrid approach called Detached Eddy Simulation 
(DES), aimed at combining the features of RANS for the resolutions of the near-wall regions and LES in the separated shear layer through the resolution of the turbulent structures. The main issues limiting the application of the standard formulation of DES model are related to the development of the unsteady turbulent structures switching from the RANS to the LES model and the premature activation of the LES model in attached boundary layers due to the local grid resolution, a phenomenon addressed as Grid Induced Separation (GIS).

In order to prevent the activation of the switching criterion, several approaches were proposed through the introduction of blending functions to shield the boundary layers from the DES limiter, resulting in the terminology Delayed Detached Eddy Simulation (DDES). A further refinement is provided by the Improved DDES (IDDES) model, which extends the LES zone to the outer part of the wall boundary layers. The formulation implemented in CFX is based on the SST-DES model by Strelets 63, which switches from SST-RANS to LES where the turbulent length $L_{t}$ predicted by the RANS model exceeds the local grid spacing $\Delta$. More practically, a multiplier $F_{D E S}$ is applied to the destruction term in the k-equation:

$$
F_{D E S}=\max \left(\frac{L_{t}}{C_{D E S} \Delta}, 1\right)
$$

The mechanisms used in CFX to prevent the GIS in the attached portion of the boundary layer is based on the modification in the above mentioned limiter, exploiting the blending functions of the SST turbulence model $F_{1}$ and $F_{2}$.

$$
F_{D E S-C F X}=\max \left(\frac{L_{t}}{C_{D E S} \Delta}\left(1-F_{S S T}\right), 1\right) ; \text { with } F_{S S T}=0, F_{1}, F_{2}
$$

The default choice is represented by $F_{S S T}=F_{2}$, since it provides a greater shielding of the boundary layer, whereas with $F_{S S T}=0$ the standard formulation by Strelets [63] is recovered. According to 98] this formulation would reduce the GIS problem by an order of magnitude. 


\subsubsection{Turbulence modelling - Scale Adaptive Simulation}

The Scale-Adaptive Simulation (SAS) represents a second generation URANS formulation, which allows the resolution of the turbulent spectrum of unstable flows. The SAS concept is based on the introduction of the von Karman length scale $L_{v K}$ into the turbulence equations, so as to dynamically adjust the resolved structures and reduce the eddy viscosity in such regions.

$$
L_{v K}=\kappa\left|\frac{U^{\prime}}{U^{\prime \prime}}\right| ;\left|U^{\prime}\right|=\sqrt{\frac{\partial U_{i}}{\partial x_{j}} \frac{\partial U_{i}}{\partial x_{j}}} ;\left|U^{\prime \prime}\right|=\sqrt{\frac{\partial^{2} U_{i}}{\partial x_{j}^{2}} \frac{\partial U_{i}}{\partial x_{k}^{2}}} ;
$$

The SAS model remains in RANS mode in zones characterized by low instability, while it provides LES-like results in the unsteady regions of the flow field. The RANS part of the model results unaffected by the grid spacing, preventing the issues related to the GIS. Therefore, unlike DES, the model can be employed to perform simulations on a much wider range of numerical grids. However, if the computational grid and/or the time step is not appropriate for LES, the model will remain in RANS mode.

For what concerns the implementation in CFX, the formulation made available in the solver consists in the the latest version of the SST-SAS model 99]. The governing equations of this model differ from those of the SST RANS model by an additional SAS source term in the $\omega$-equation.

\subsubsection{Results}

\subsubsection{Flow field characteristics}

The characteristics of the flow field on the center plane, generated by the injector for the reference working conditions $\Delta P / P=3.5 \%$, are reported in Fig. 3.30 The main features of swirled flow are the corner vortex and the central recirculation generated by the vortex breakdown. The jet exiting from the swirler impinges on the liner wall at about $x / D=0.7$. Slightly downstream, at $x / D=1.1$ and $z / D=0.5$, a low velocity zone can be observed; in this position, corresponding to the 
maximum extent of the Inner recirculation zone (IRZ), the impinging jet near the wall is accelerated, resulting in a high axial velocity gradient. Furthermore, the picture points out also the typical Outer recirculation zone (ORZ) established in the proximity of the corner. The measured velocity field indicates that the coolant issued by the slot is early lifted up by the backward recirculating flow (light blue area near the lateral wall), which reasonably provides a scarce protection to the liner.

For what concerns the CFD simulations, an overall good agreement is achieved among experimental and numerical data, even though some discrepancies can be observed: the opening angle of the swirling flow obtained by SAS runs seems greater, leading to a shift of the stagnation point towards the dome and a reduction in the extension of the corner vortex, that, as a consequence, increases the intensity of the recirculation. On the contrary, the DES1 case shows the tendency to return a lower opening angle, that extends the high speed region more downstream. Nevertheless, it is interesting to notice that the differences between the two turbulence models are significantly reduced when the cases with the refined mesh are considered ( $S A S 2$ and DES2). Moreover, the mesh refinement allows a better prediction also for what concerns the velocity magnitude within the IRZ.

With the intent of providing a more quantitative comparison, the profiles of axial velocity were extracted at increasing stream-wise distance. The overall good agreement with experimental data is confirmed in Fig. 3.31 the CFD profiles are roughly coincident at $x / D=0.04$, where it is possible to notice the two velocity peaks due to the flows coming from inner and outer swirlers, whereas at $x / D=0.24$ they are merged within a unique swirling structure. Beyond this position the profiles between the two models start to diverge, with the DES model that shows an overprediction in the velocity magnitude and a lower opening angle, whereas the SAS closely matches the experimental data. The effect related to the mesh refinement is not significant, but further improves the agreement between SAS and experimental data.

With the purpose of showing the role played by the models in the 


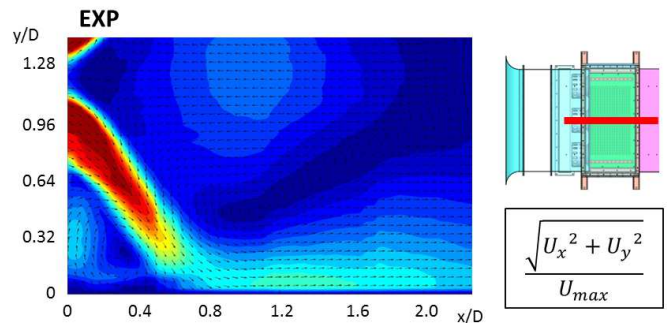

SAS 1

$\mathrm{U} / \mathrm{U}_{\max }$

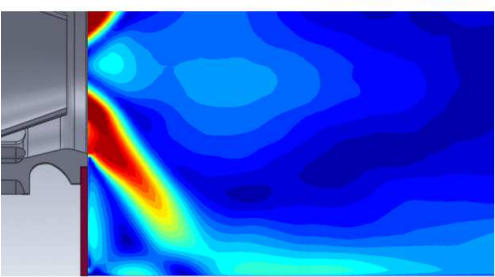

SAS 2

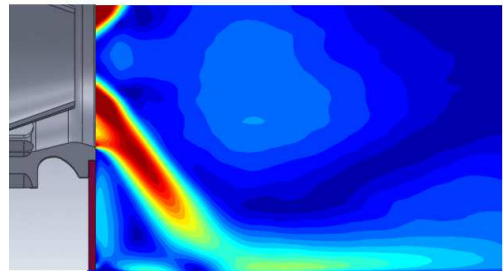

Figure 3.30: Flow field on the center plane

DES 1

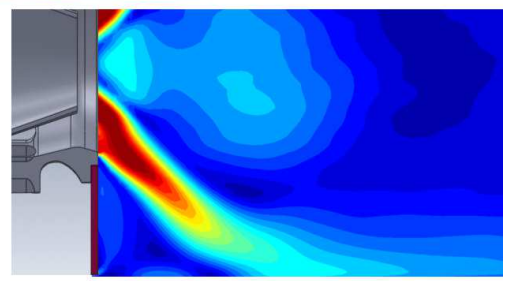

DES 2

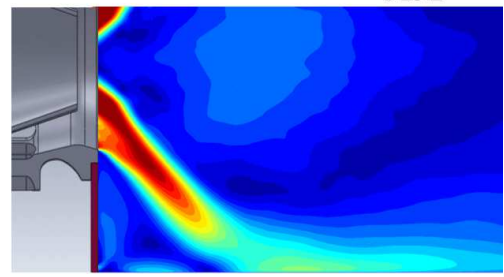

resolution of the turbulence field, as well as the impact of mesh refinement on the results, the $M$ criterion proposed by Pope 100 was calculated. This parameter expresses the ratio between modelled and total (modelled + resolved) turbulence kinetic energy and it can be considered a representative index of the turbulence-resolution, e.g. $M=0.2$ corresponds to the resolution of $80 \%$ of the kinetic energy. Even though the local value of $M$ is affected by the value of the mesh size at other locations and at earlier times, this criterion is currently one of the most popular methods to quantify the quality of Large Eddy Simulations in predicting 

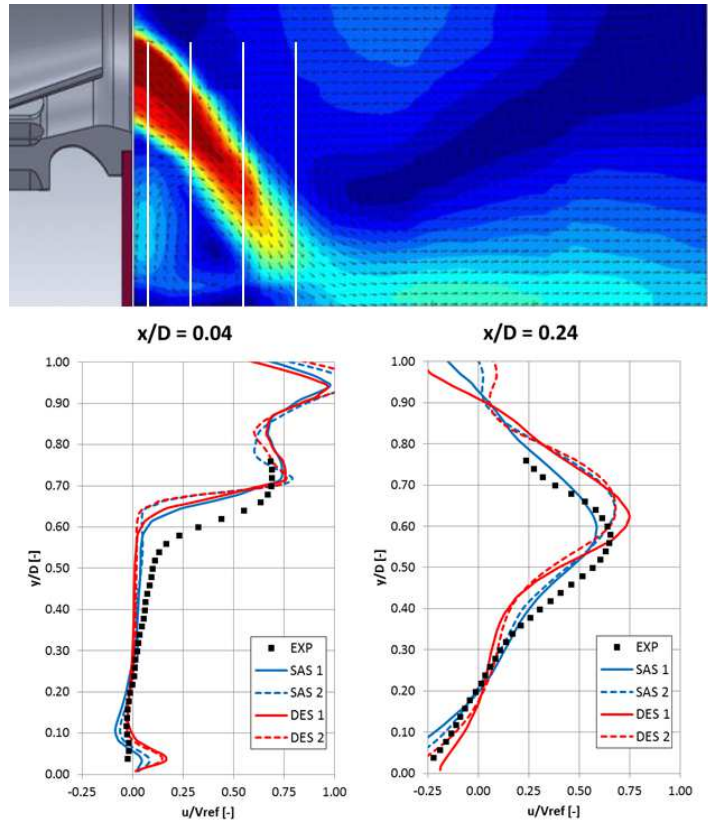

$x / D=0.24$
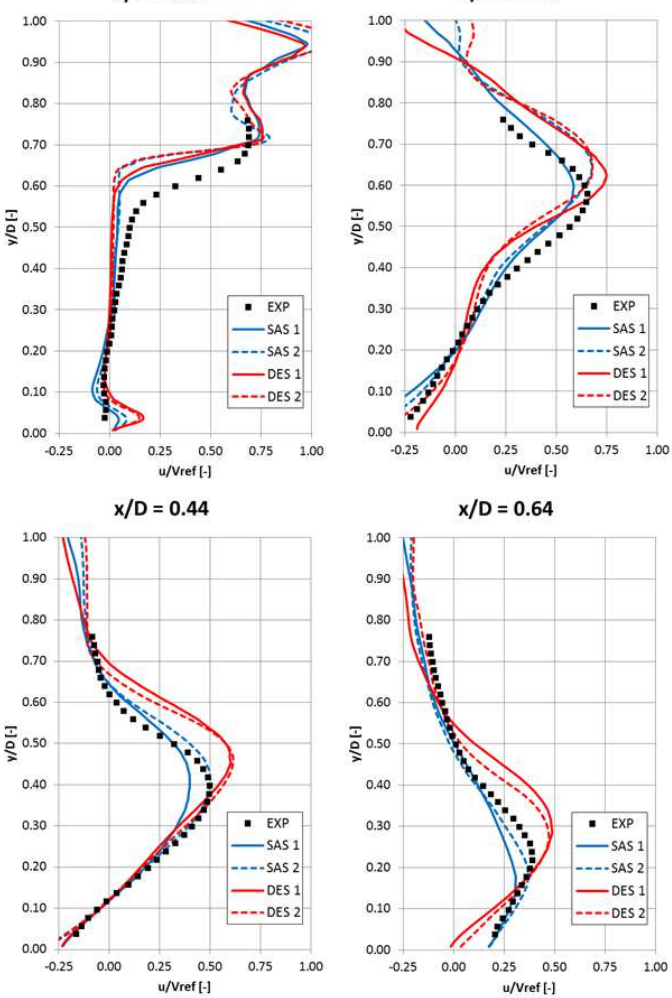

Figure 3.31: Profiles of velocity component in stream-wise direction (center plane) 
the velocity field 101 .

Fig. 3.32 reports the $M$ distribution for the cases under consideration. As it possible to notice, the SAS model returns a sufficient turbulence resolution in the most part of the combustor, and the average value of $M$ further decreases with mesh refinement, whereas the solution is instead mostly RANS in the proximity of the swirler walls (not shown in the picture). This effect can be ascribed to the nature of the SAS model, that is suited for globally unstable flows and is not able to switch from RANS to a LES-like behaviour in absence of large and unstable separation zones 96.

On the contrary, the DES cases show a significant part of the domain characterized by $M \approx 1$. The wall boundary layers are in fact entirely covered by the RANS model, hence explaining the large amount of modelled turbulence kinetic energy, whereas the LES mode is achieved only in the detached regions away from walls. The extension of the RANS modelled region reduces with mesh refinement, pointing out that locally the grid and time step resolution are sufficient to activate the DES limiter. Nevertheless it would be interesting to assess the impact of different formulations for the shielding function implemented in more advanced versions of the DES model, such as DDES and IDDES.

In order to better understand the features of the flow field, the instantaneous velocity field is reported in Fig. 3.33 The results obtained by $S A S M 2$ were employed for this purpose, since this turbulence model pointed out the best agreement with PIV measurements. The Precessing vortex core (PVC), highlighted by a constant pressure isosurface, generates a strong unsteadiness and enhances the mixing in the shear layer between the swirling flow and the low velocity air of the IRZ. Despite the difficulties in understanding the unsteady behaviour of the flow field from the picture, it is anyway evident that the opening angle leads to a significant interaction through the periodicity boundaries, with the migration of very large turbulent structures. For this reason, it could be interesting to perform a simulation of the entire three-sector rig, so as to evaluate the effect of the assumption of periodicity. 
SAS 1

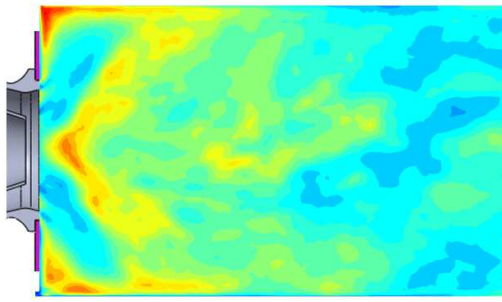

SAS 2

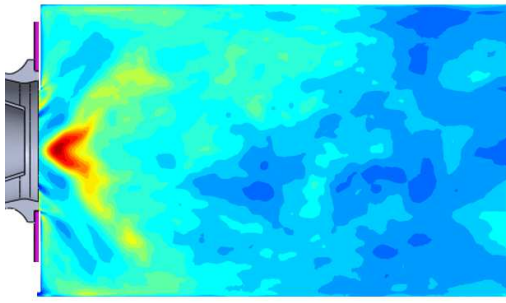

$\begin{array}{lllllllllll}0.0 & 0.04 & 0.08 & 0.12 & 0.16 & 0.20 & 0.24 & 0.28 & 0.32 & 0.36 & 0.40\end{array}$
DES 1

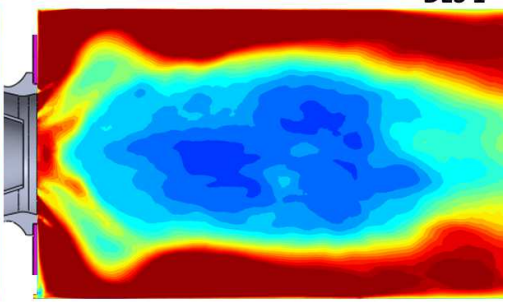

DES 2

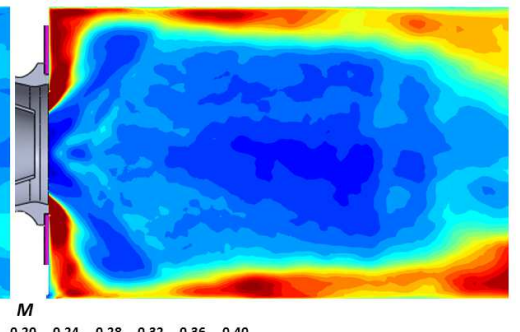

Figure 3.32: Effect of mesh refinement and turbulence modelling on $M$ criterion (center plane)

\subsubsection{Heat transfer augmentation}

Fig. 3.34 displays, for the central sector of the test section, the estimated Nusselt number distribution. As a general result, maps point out the presence of an elliptic area where the heat transfer coefficient reaches the peak values. A slight shift of this area on the right side of the center of the swirler is coherent with the sense of rotation of the swirlers. As pointed out by the PIV results at the reference condition, this zone, located between $x / D=0.4-0.8$, corresponds to the location of stagnation point of the impinging jet and the following strong acceleration of the flow near the wall. Low heat transfer values are registered in the other zone of the test plate, especially in the corner recirculation zone.

Moreover, pictures in Fig. 3.34 highlight a non symmetric distribution 


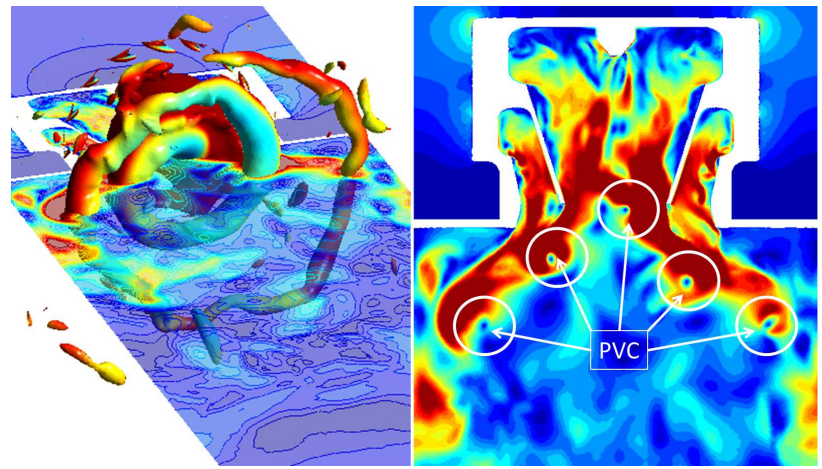

Figure 3.33: 2D velocity on the median plane: PVC visualized by a constant pressure isosurface and coloured by stream-wise velocity

of HTC in the proximity of the slot exit, due to a non uniform flow field near the wall: in fact the entire test section is affected by a macro flow structure which rotates around the three injectors and tends to direct towards the right side the flow near the test surface (towards the left on the opposite side of the test section). The sense of rotation of this macro recirculation is concordant with that impressed to the flow by each swirler as depicted in Fig. 3.35

For what concerns the numerical results, SAS model shows a general good agreement, even though the size of the impingement region appears slightly over-predicted, especially regarding the peak level of $N u / N u_{0}$. The above mentioned non uniform coolant injection is present, despite the location of the region characterized by the maximum protection is shifted on the right. It is worth noticing that the experimental map does not appear perfectly periodic, suggesting again to further investigate the effects of the periodicity assumption on the CFD result. Moving to the Nusselt number distribution obtained by the DES $M 1$ run, the overprediction appears this time huge and extended even more downstream, whereas the region immediately close to the dome agrees quite well with the experimental map. It is worth pointing out that the mesh refinement, 
which does not involve a significant impact with the SAS model, deeply affect the results obtained with the DES model. This phenomenon can be explained in the light of the results previously shown in Fig. 3.32 with relation to the variation in the extension of the shielded region depending on the mesh size. In fact, whereas the SAS model gradually shift to a LES-like behaviour reducing the mesh size, the turbulence modelling through DES involves a sudden switch from RANS to LES through the shielded region.

To better understand the quantitative differences among the simulations, the laterally averaged trends of $N u / N u_{0}$ along the stream-wise direction were computed and reported in Fig. 3.36 . As highlighted by the $2 \mathrm{D}$ distributions, the experimental heat transfer coefficient is low at the entrance of the test section, but quickly reaches a maximum, after which the magnitude decays slowly. Both SAS cases accurately reproduce the experimental trend, especially downstream of the impingement region, where the error lies below the experimental uncertainty. The peak of Nusselt is slightly over-predicted and shifted upstream for $S A S M 1$ (but accurately reproduced by $S A S M 2$ ), due to the different opening angle of the swirling flow and the resulting effects on the corner vortex, that lead to a reduction in agreement. The profile calculated by the DES runs constantly overestimate the experimental data (up to 50\%), even though the axial position of the peak is correctly reproduced, whereas the better agreement achieved for DES M2 case (see Fig. 3.34 is considerably hidden by the smoothing effect caused by the lateral averaging process.

\subsubsection{Remarks}

A numerical investigation was carried out in order to evaluate the capability of hybrid RANS-LES models in predicting the flow field and the heat transfer in a in a non-reactive three sector planar rig equipped with three injectors. The rig is characterized by a simple cooling scheme made up of a slot injection, reproducing the exhaust dome cooling mass flow.

The analysis of the flow field has highlighted a general good agreement, 


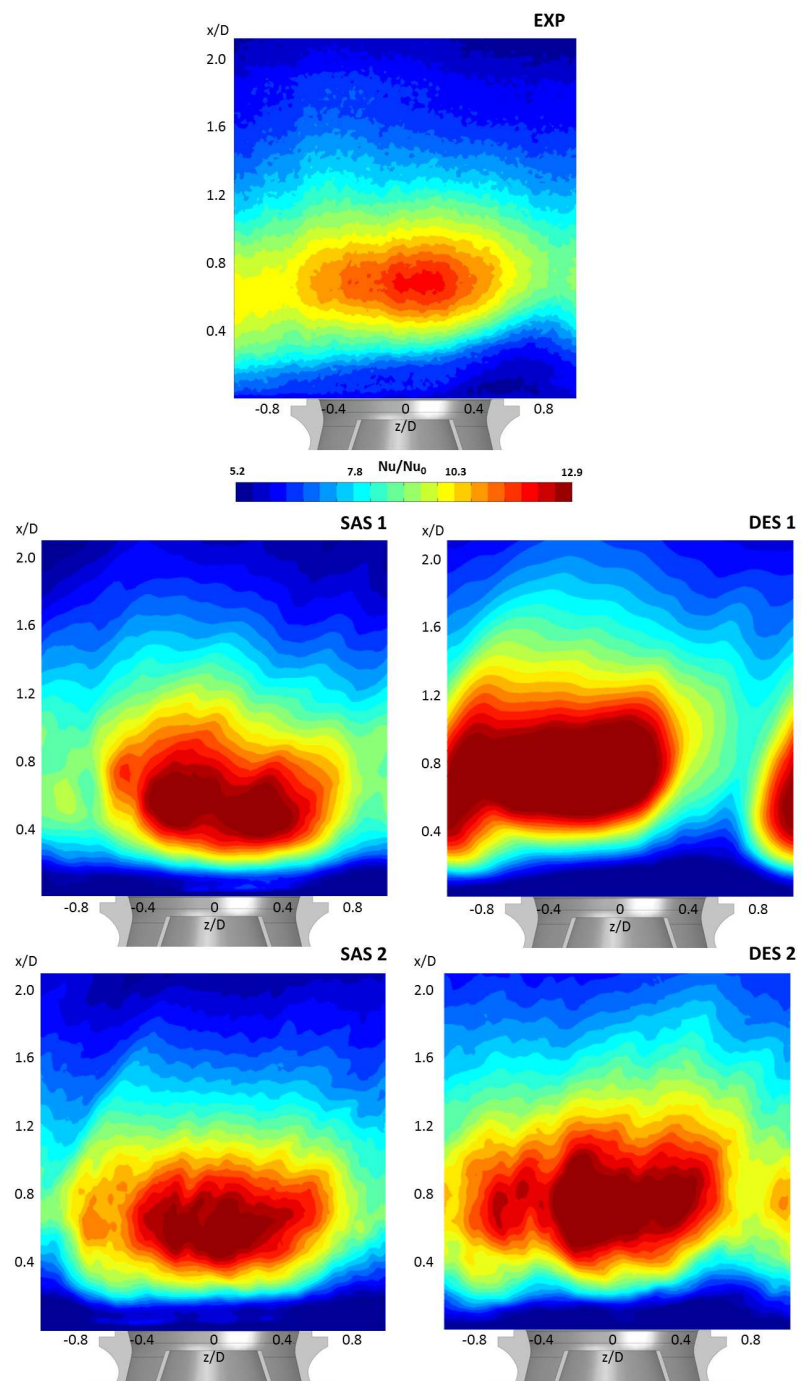

Figure 3.34: Nusselt number distributions on the central sector 


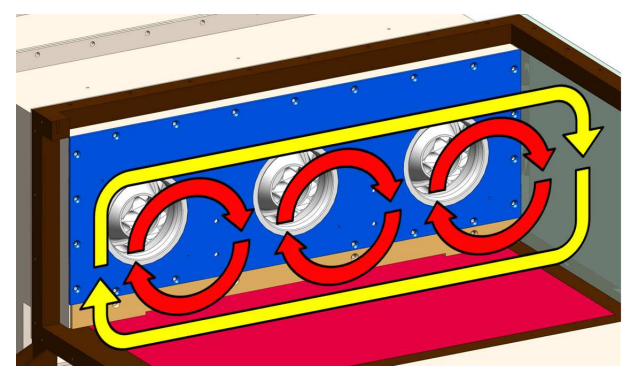

Figure 3.35: Sketch of the main recirculation structures inside the test section

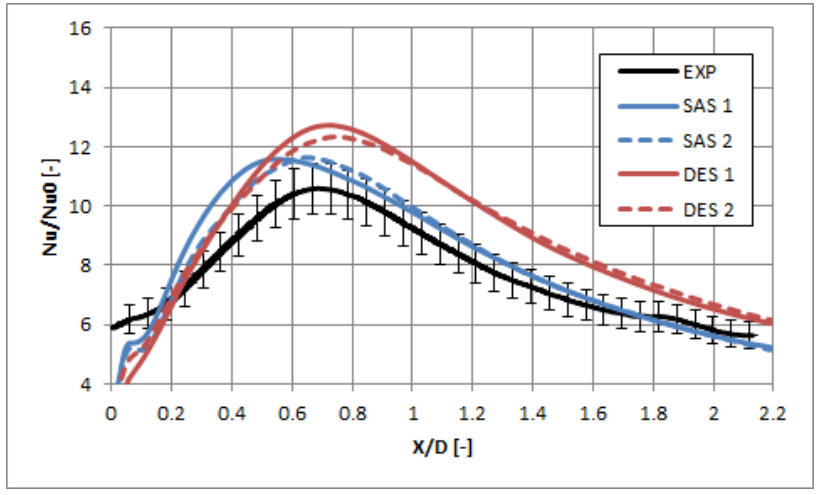

Figure 3.36: Laterally averaged Nusselt number augmentation

characterized by some discrepancies in the opening angle of the swirling flow that alter the location of the corner vortex. The SAS model shows the best agreement with PIV data, whereas DES returns a greater acceleration in the near-wall region downstream of the impingement region. These effects have a direct impact on the resulting heat transfer estimation: the $N u / N u_{0}$ distribution obtained with the SAS agrees very well, especially the location of the peak, where the error lies below the experimental uncertainty, whereas the DES run constantly over-predicts the magnitude 
of the heat transfer. On equal mesh, the behaviour of the shielding function implemented in the DES model appears conservative, with the wall boundary layers entirely modelled by RANS, hence suggesting the opportunity of assessing more advanced formulations such as DDES and IDDES.

The strong interaction across the lateral boundaries highlighted by the unsteady flow field, as well as the non perfect uniformity of the experimental Nusselt distribution, would recommend to investigate the periodicity assumption exploited to simulate a single injector. Moreover, the promising results obtained with the SAS model represent a starting point for further investigations that take into account the presence of effusion cooling. An experimental campaign on this more representative configuration has been already carried out and the main findings of which are reported by Andreini et al. [102, 103].

\subsection{Concluding remarks}

This chapter presented the main findings of three CFD campaigns aimed at investigating the impact of the swirling flow on the performance of effusion cooling liners. The numerical setup was validated against experimental measurements of adiabatic effectiveness carried out on flat plates. The comparison highlighted a huge improvement ascribable to the application of a modified version of the standard SST model to account for the anisotropy of turbulence in the near-wall region, even though slight discrepancies persist at low Blowing Ratio (i.e. out of the penetration regime), for which small errors in the prediction of the jet trajectory can lead to huge errors in the estimation of film coverage.

The same setup was then exploited on a more representative test case consisting of a tubular combustor, equipped with a lean burn injector to provide a realistic flow field. Both orthogonal $\left(90^{\circ}\right)$ and slanted $\left(30^{\circ}\right)$ perforations were investigated, pointing out a sort of two-way interaction between swirling flow and film cooling, where also the hole inclination angle plays a key role in modifying the characteristics of the outer recirculation 
region, thus affecting the coolant protection in the first part of the liner.

Despite the importance of adiabatic effectiveness, an accurate assessment of convective heat loads requires also the estimation of the heat transfer coefficient distribution. The CFD tool was then benchmarked on a linear three-sector rig representative of a lean burn slot cooled annular combustor, without taking into account the effusion cooled liners due to the large number of holes. The (U)RANS approach returned a not physical flow field, therefore hybrid RANS-LES models were tested, which provided a very good agreement in predicting the measured flow field and Nusselt number distribution (error below 10\%).

Therefore, the numerical approach showed overall promising results, even if the approach is not feasible for the investigation of effusion cooled combustors, that would imply an unbearable increase in the computational effort. With the purpose of overcome this issue, an interesting solution could be represented by the modelling of effusion cooling holes, so as to avoid the meshing of discrete holes. Thus, an innovative model was developed and tested, as described in Chapter 4 


\section{Chapter 4}

\section{Development and Validation of a Film Cooling Model}

\subsection{Introduction}

As a result of the exponential growth in computational resources occurred in the last decades, CFD has become a viable and reliable tool for the design of gas turbine combustors. Even though the numerical approach can not be considered mature enough to completely dispose of the support and validation provided by experimental measurements, an increasing number of procedures for the combustor design are based on CFD simulations. Steady RANS can provide sufficiently accurate prediction of the flow and temperature fields within the combustion chamber at an affordable computational cost.

Unfortunately, the context is worsened by the introduction of effusion cooling in modern lean combustors. Considered that at least 100000 grid elements are required to provide a mesh independent solution of a single film cooling hole, the number of holes included in each sector of an annular combustor (1000-5000) makes a conjugate heat transfer (CHT) simulation of an entire combustor a challenge hardly compatible with stringent time requirements typical of industry. To the purpose of improving the feasibility of this kind of investigation, many authors proposed several 
approaches based on the modelling of film cooling injection, so as to avoid the meshing of discrete holes.

\subsection{State-of-the-art}

To the authors' knowledge, one of the first attempt can be attributed to Crawford et al. 104, who implemented a model for the coolant injection in a 2D boundary layer code. Heidmann and Hunter 105 applied a model, previously developed by Hunter [106, based on distributed volumetric sources of mass, momentum, energy and turbulence quantities, pointing out the strong sensitivity to mesh refinement. Tartinville and Hirsch 107. developed an algorithm that, starting from the geometrical features of the hole, detected the cells located at the hole discharge on which to apply requested source terms. Burdet et al. 108 developed a model embedded in a CFD code that, taking into account the blockage effect due to the coolant jet, was based on the injection through a plane located just after the hole trailing edge. Recently auf dem Kampe and Völker [109] proposed the addition of source terms in a volume, whose extent depends on film cooling parameters. The model was implemented in the commercial code ANSYS CFX and compared to detailed CFD simulations and experimental data, highlighting good results even with very coarse grids.

The approach proposed by Mendez and Nicoud [110] is quite different compared to previous ones. This model, specially conceived for effusion cooling liners, does not lie as much in the addition of local source term, but rather in application of homogeneous boundary conditions on both aspiration and injection side, hence permitting the use of coarse grids. The validation, performed comparing numerical and experimental velocity profiles, highlighted a good reproduction of the global structures.

Another innovative solution was developed by Rida et al. [111, which reported that up to a million cells is required for a single hole to reach the mesh independence for an accurate prediction of the flow structures within the perforation. According to the authors, a feasible method, alternative 
to the addition of source terms, is hence represented by imprinted effusion modelling which consists in coupling cold and hot sides of the effusion orifice with a outlet and inlet boundary conditions, without meshing the hole. The mass flow rate is calculated locally starting from the pressure drop and a correlation-based calculation of $C_{D}$. A comparison with RANS and LES simulations including the effusion holes picked out a good agreement for flow field, mass flow rate and adiabatic wall temperature.

Recently, Voigt et al. 112 pointed out the lack of models that take into account the thermal power removal within the hole due to convection, a necessary feature in order to perform a conjugate heat transfer (CHT) calculation. They used CFX, which permits to apply local source terms to model coolant aspiration and injection, as well as heat sink in the metal. Such approach implies the necessity to specify source values, in terms of mass flow rate, velocity components, total temperature and turbulence quantities, in addition to heat sink, which can be calculated with a preliminary detailed simulation or through a correlative approach.

The main goal of this work is represented by the implementation of a local pressure drop dependent formulation that allows the automatic calculation of the mass flow rate through each hole and the heat exchanged due to heat sink. The main advantage of this methodology is that no detailed simulation with discrete holes is required a priori, hence significantly reducing the computational effort.

\subsection{Model development}

The model presented in this work, called SAFE (ㅇorce based effusion model), has been developed to work with ANSYS CFX. As already said, the software allows the application of source points within the domain, acting as a source if a positive mass flow rate is applied and a sink otherwise. The main concept is therefore to apply a mass source and a mass sink in the fluid domain at discharge and entrance of a hole respectively, while an energy sink can model the heat sink in the solid domain due to convective heat transfer through the hole (see Fig. 4.1. 


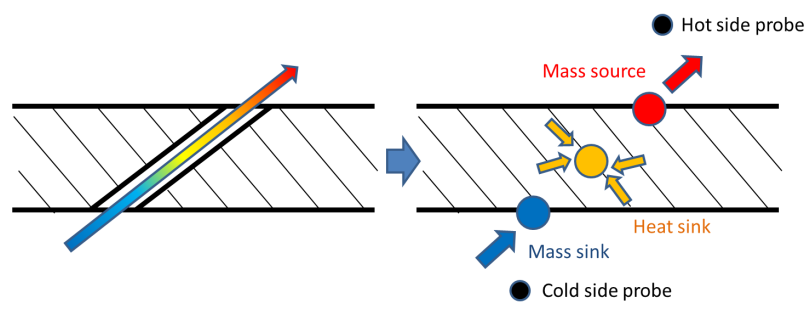

Figure 4.1: Conceptual representation of effusion hole modelling

The inputs required by the software depend on the purpose of the source point (whether mass source/sink or heat sink) and the way they are calculated have been reported below:

- Heat sink

- Heat removal due to convection inside the hole is described by Newton's law of convective heat transfer:

$$
\dot{q}=h \pi D_{h} L_{h}\left(T_{0}-T_{w}\right)
$$

- Mass source

- Mass flow rate through each hole is calculated in agreement with discharge coefficient definition:

$$
\dot{m}=C_{D} \dot{m}_{i s}
$$

where:

$$
\dot{m}_{i s}=P_{0}\left(\frac{P}{P_{0}}\right)^{\frac{\gamma+1}{2 \gamma}} \sqrt{\frac{2 \gamma}{(\gamma-1) R T_{0}}\left[\left(\frac{P_{0}}{P}\right)^{\frac{\gamma-1}{\gamma}}-1\right]} \frac{\pi}{4} D_{h}^{2}
$$

The calculation of coolant and gas conditions required for the assessment of isentropic mass flow rate is performed by runtime measurements from probe points (see Fig. 4.2). Cold side 
probes, located in annulus/plenum, measure total quantities of temperature and pressure, while hot side probes, located in mainstream, provide static pressure values.

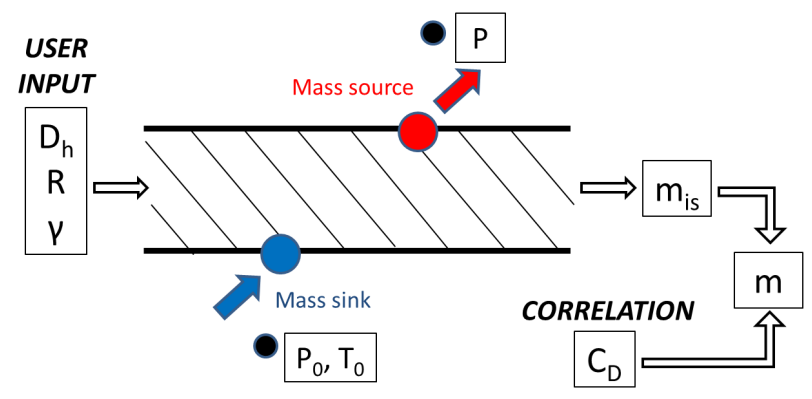

Figure 4.2: Sketch of mass flow rate calculation

- Discharge temperature takes into account the heating of the fluid through the hole due to convection:

$$
T_{\text {out }}=T_{0 \text { in }}-\frac{\dot{q}}{\dot{m} c_{p}}
$$

- Velocity components are derived from velocity at hole discharge and hole inclination angle and, within the software, can be expressed either in cartesian or cylindrical components:

$$
\begin{gathered}
V_{x}=V \cos \alpha ; V_{y}=V \sin \alpha ; V_{z}=0 \\
V_{\text {axi }}=V \cos \alpha ; V_{\text {tan }}=0 ; V_{\text {rad }}=V \sin \alpha \\
V=\frac{\dot{m}}{\rho_{\text {out }} \frac{\pi}{4} D_{h}^{2}}=\frac{\dot{m}}{\frac{P}{R T_{\text {out }}} \frac{\pi}{4} D_{h}^{2}} \approx \frac{\dot{m}}{\frac{P}{R T_{0} \text { out }} \frac{\pi}{4} D_{h}^{2}}
\end{gathered}
$$

where the density in velocity expression is calculated with total temperature at hole discharge, since static temperature is not known. This assumption is acceptable in aeroengine combustors, since Mach number is relatively low, compared to perforations of gas turbine nozzles and blades. 
- Required turbulence quantities depend on the employed turbulence model ( $\mathrm{k}-\omega$ in this particular case). To the authors' knowledge no correlations are available in literature to estimate turbulence quantities at hole discharge, therefore they are calculated, referring to CFX manual [113, assuming a turbulence intensity $T u$ value at hole discharge:

$$
k=\frac{3}{2} V^{2} T u^{2} ; \varepsilon=\frac{k^{3 / 2}}{0.3 D_{h}} ; \omega=\frac{\varepsilon}{k}
$$

In case of mass sink only the mass flow rate needs to be specified, since all the other physical quantities are transported out of the domain with local values.

This methodology reduces the number of unknowns and hence user inputs. Thus the only parameters required are:

- Gas properties: specific gas constant R, specific heat capacity at constant pressure $c_{p}$ and heat capacity ratio $\gamma$;

- Drilling features: hole diameter $D_{h}$, hole length $L_{h}$ and inclination angle $\alpha$;

- Fluid dynamic parameters: turbulence intensity $T u$, heat transfer coefficient $h$ and discharge coefficient $C_{D}$.

While the value of turbulence intensity $T u$ must be assumed, the last two parameters can be estimated by means of a wide range of correlations. As far as this work is concerned, $h$ is calculated through Cho and Goldstein correlation for short length holes [114, valid in a range of $1300 \leq R e \leq$ 30000:

$$
N u=0.0397 \operatorname{Re}^{0.73} \operatorname{Pr}^{0.33}\left(1+2.384\left(\frac{L}{D}\right)^{-0.621}\right)
$$

Discharge coefficients, on the other hand, are calculated by a CFDbased correlation for effusion cooling holes developed by Andreini et al. [115], where the value of $C_{D}$ of each hole is calculated as: 


$$
C_{D, i}=a_{0}+a_{1} \cdot \dot{m}_{r} \cdot\left(1+a_{2} \cdot V_{h r, i}\right)+a_{3} \cdot \frac{R e_{a n, i}}{R e_{h, i}}
$$

Velocity head ratio $V_{h r}=\frac{P_{0, a n}-P_{a n}}{P_{0, a n}-P_{g}}$, as well as flow coefficient $\dot{m}_{r}=$ $\frac{\dot{m}_{a n, i n} \sqrt{R T_{0}}}{P_{0} A}$ and annulus Reynolds number $R e_{a n}$ can be easily calculated, whereas hole Reynolds number $\operatorname{Re}_{h}$ (required also for the estimation of $h$ ) is dependent on hole mass flow rate, which is unknown a priori and cannot be calculated iteratively by CFX using simple algebraic expressions. Therefore it has been decided to formulate $\dot{m}=C_{D}^{\prime} \cdot \dot{m}_{i s}$, where $C_{D}^{\prime}$ is a first attempt value of the discharge coefficient.

\subsection{Model validation}

\subsubsection{Experimental test case}

In order to assess the accuracy of the proposed methodology a comparison with an experimental data set of overall effectiveness will be discussed. Such a data set has been obtained by an extensive experimental analysis aiming at the investigation of thermal performance of flat effusion plates. In particular, in this paper are reported only experimental results of three plates, differentiated by the hole diameter, whose geometric features are reported in Tab. 4.1. test plates are realized with a scaling up factor of 3 with respect to actual engine reference. Furthermore, each plate has been tested with several blowing ratio conditions $(\mathrm{M}=1.5,3,5)$. For further details please refer to Andreini et al. [116].

\begin{tabular}{ccccccc}
\hline & $D[\mathrm{~mm}]$ & Rows & $L / D[]$ & $\alpha[\mathrm{deg}]$ & $S_{x} / D[]$ & $S_{y} / D[]$ \\
\hline G1 & 1.88 & 18 & 4.99 & 30 & 7.30 & 5.88 \\
G2 & 1.50 & 18 & 6.25 & 30 & 9.15 & 7.37 \\
G3 & 2.34 & 18 & 4.01 & 30 & 5.86 & 4.73 \\
\hline
\end{tabular}

Table 4.1: Test case outline

Concerning the test rig, the mainstream enters at about $60^{\circ} \mathrm{C}$ through a square section channel, which is equipped with a turbulence generator 
grid at the entrance that provides the required turbulence intensity level $(17 \%)$, while coolant is fed by a plenum at about $20^{\circ} \mathrm{C}$. A set of thermocouples, embedded in the metal and located along the stream-wise direction, permits the measurement of metal temperature, so as to allow the evaluation of overall effectiveness.

\subsubsection{Numerical setup}

3D CFD RANS conjugate calculations have been considered in this study, referring to the Navier-Stokes equations solver ANSYS CFX v14. The mainstream boundary conditions have been assigned in terms of mass flow rate, total temperature and turbulence intensity at the inlet, static pressure at the outlet. Mass flow rate and total temperature have been imposed at the inlet plenum.

Compressibility effects have been taken into account and a High Resolution advection scheme has been used, which automatically blends between first and second order backward Euler scheme throughout the domain based on the local solution field 113. The fluid has been modeled as ideal gas, thermal conductivity has been specified for the plate material. Energy equation has been solved in terms of total energy and viscous heating effects have been accounted for. The k- $\omega$ SST turbulence model, in its formulation made available by the CFD solver, has been used in conjunction with an Automatic near-wall treatment approach, that blends between Wall Function and Wall Integration on the basis of $y^{+}$value. For the cases presented in this work where $\max \left(y^{+}\right)<1$, the Low Reynolds formulation is always recovered.

ANSYS ICEM-CFD has been used to generate hybrid computational grids (tetrahedral with 15 layers of prisms close to the wall). The fluid domain grids count about 3 million elements, while the solid domain grids about 0.35 million elements. The number of elements decreases significantly modeling the effusion holes with local sources, for which half plate has been simulated. In case of detailed simulations with discrete holes the computational domain has been simplified taking advantage of symmetry condition and simulating only half hole for each row (see 
Fig. 4.3). Adiabatic conditions have been imposed at upstream and downstream extremities of the plate. The solution convergence has been assessed by monitoring the residuals and stopping the simulations when the trend of minimum, medium and maximum temperature of the plate reached a steady-state.

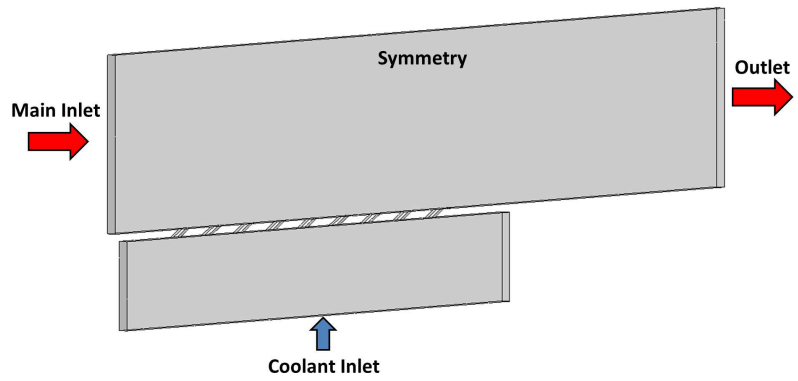

Figure 4.3: Sketch of the computational domain for detailed simulations

\subsubsection{Results: Sensitivity analyses}

\subsubsection{Detailed simulations}

As previously mentioned, the input data for the model based on prescribed values have been obtained from $\mathrm{CHT}$ simulations with discrete holes, whose results for $M=5$ are here reported. In the following figures experimental data are reported by dots, whereas numerical data are depicted by dashed lines. Coherently with experiments, temperature is calculated in the same location of thermocouples, therefore any discontinuity in the curves must be ascribed to the linear interpolation of punctual values. As far as the axis of abscissae is concerned, the stream-wise distance $x$ has been normalized by the stream-wise pitch $S_{x}$, so as to obtain the first row of holes for $x / S_{x}=1$, the second one for $x / S_{x}=2$ and so on.

In Fig. 4.4 the comparison of $\eta_{o v}$ between experimental data and detailed simulations is reported, where the axial distance is divided by 
the stream-wise pitch so as to obtain the first row at $x / S_{x}$ and so on. It is possible to notice a general agreement for all three geometries, that improves in the last part of the plate, where coolant superposition leads to a better protection from hot gas and hence to higher overall effectiveness. The underprediction nearby the first rows can be ascribed to $\mathrm{k}-\omega \mathrm{SST}$ model, which is based on the turbulence isotropy assumption, that leads to a strong underestimation of adiabatic effectiveness in this area, as reported by Andrei et al. [117.

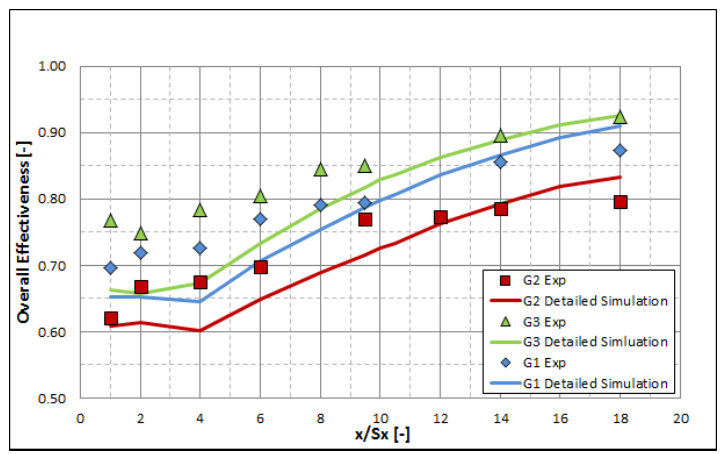

Figure 4.4: Results for detailed simulations $(M=5)$

As previously stated, the post-processing of these detailed simulations has provided the required input data for source points. In particular, mass flow, total temperature, velocity components, turbulence quantities, as well as heat transfer rate due to heat sink, have been averaged over the whole perforation and applied locally on each hole.

\subsubsection{Sensitivity to surface mesh size}

In order to investigate the effect of this parameter, four different grids have been generated on varying the triangular $2 \mathrm{D}$ elements at wall, which is named Max Size $(M S)$ in ICEM-CFD, in steps of $0.25 \mathrm{~mm}$ (see Tab. 4.2.

In Fig. 4.5 the trends of overall effectiveness for experimental data, 


\begin{tabular}{cccccc}
\hline$M S[\mathrm{~mm}]$ & 0.75 & 1.00 & 1.25 & 1.50 & 1.75 \\
\hline Elements & $2.3 \cdot 10^{6}$ & $1.6 \cdot 10^{6}$ & $1.3 \cdot 10^{6}$ & $1.1 \cdot 10^{6}$ & $1.0 \cdot 10^{6}$ \\
\hline
\end{tabular}

Table 4.2: Grids used in the assessment of the sensitivity to $2 D$ element dimension

detailed simulations with discrete holes and source-based model are compared (geometry G2 only is reported, since similar results have been obtained for the other geometries). Comparing the results for the three geometry, it is possible to notice that the best agreement can be achieved with a size of $50 \%$ of hole diameter (see Tab. 4.3, confirming what obtained by Voigt et al. [112.

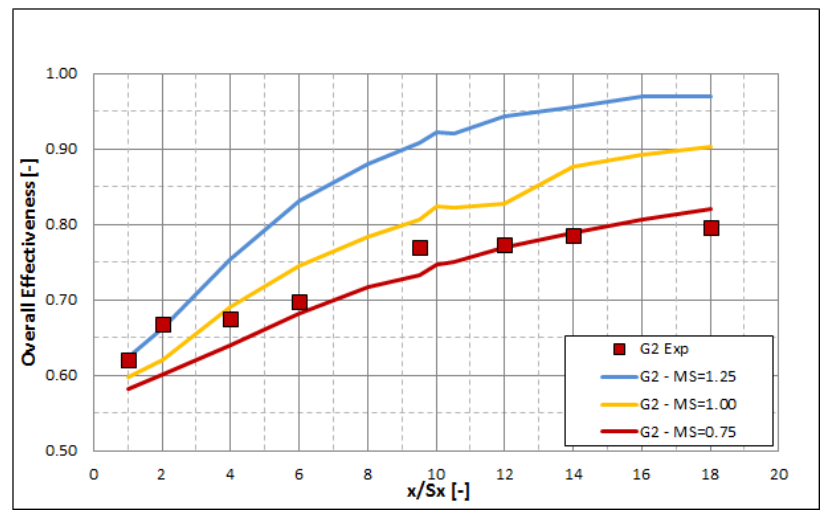

Figure 4.5: Effect of Max Size $(G 2, M=5)$

\begin{tabular}{clll}
\hline Case $\left(D_{h}[\mathrm{~mm}]\right)$ & \multicolumn{3}{c}{$M S[\mathrm{~mm}]\left(M S / D_{h}[\%]\right)$} \\
\hline$G 1(1.88)$ & $1.00(53)$ & $1.25(66)$ & $1.50(80)$ \\
$G 2(1.50)$ & $0.75(50)$ & $1.00(67)$ & $1.25(83)$ \\
$G 3(2.34)$ & $1.25(53)$ & $1.50(64)$ & $1.75(75)$ \\
\hline
\end{tabular}

Table 4.3: Outline of $M S / D_{h}$ values for each grid used 
The underestimation in the first part, as well as the downstream overestimation, can be justified remembering that in this phase the same averaged value of heat sink have been applied on each hole. Furthermore, since the value of heat sink applied is the same for each hole and each simulation, the difference between the runs can be attributed to numerical dissipation of the coolant jets due to near wall mesh refinement and the way source is applied. In fact, it is worth remembering that, in the particular case of CFX, the source is applied to the nearest node to the assigned position. Since the code is vertex-centered, the node of a triangle corresponds to the centroid of the polygon (see Fig. 4.6). It is possible to prove that, in order to achieve an area of the finite volume face equal to the cross-section of the hole, the Max Size of the wall mesh element must be imposed equal to about $0.47 D_{h}$.

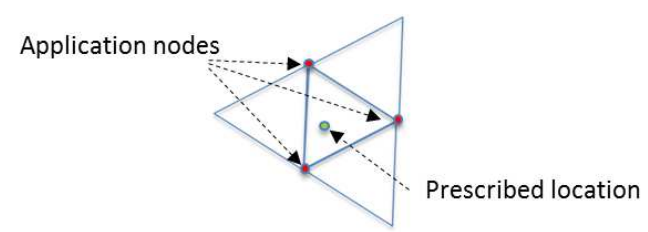

Figure 4.6: Sketch of source point application in CFX

\subsubsection{Sensitivity to volume element size}

In the same way as the previous parameter, the transition from wall mesh dimension to free-stream mesh dimension strongly influences the the discretization of the coolant jet and hence the numerical dissipation. As a consequence, a coarser mesh will cause a greater spreading of coolant jets and hence a better wall protection from hot gas. At this purpose, a comparison between experimental data and source-based simulations varying the dimension of 3D near-wall elements has been carried out. Several grids have been generated modifying the Tetra Size Ratio (TSR) parameter in ICEM-CFD (Fig. 4.7). As it is possible to notice in Fig. 4.8 a reduction of TSR seems to reduce overall effectiveness, confirming the 
significant effect of mesh refinement on jet spreading, thus improving the agreement of $M S=1.00$ curve, that approaches previous $M S=0.75$ curve. The enhancement is however limited compared to a refinement of $2 \mathrm{D}$ wall elements, leading to smaller improvement and a greater increase of the number of elements $(+50 \%)$. Therefore, in order to reduce the computational cost, it has been decided to limit the $T S R$ value to 1.2. Only the results for $G 2$ geometry have been reported, since similar conclusions can be drawn from the other geometries.

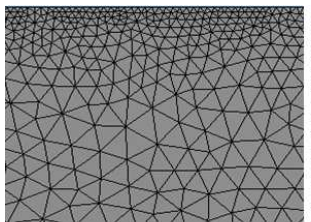

$\mathrm{TSR}=\mathbf{1 . 2 0}$

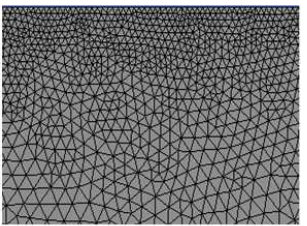

TSR $=1.05$

Figure 4.7: Effect of the parameter Tetra Size Ratio on the volume element size

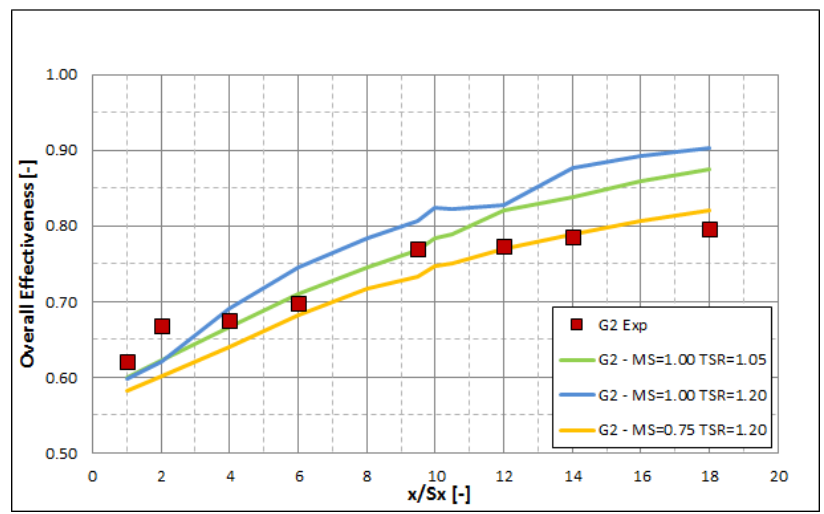

Figure 4.8: Effect of Tetra Size Ratio (G2, M=5) 


\subsubsection{Sensitivity to heat sink value}

As previously stated, constant values, derived from an averaging of required inputs on the whole perforation of the detailed simulations with discrete holes, have been applied to the source terms of each hole. This assumption is not valid in particular for the thermal power removed by convection within the holes, since in proximity of the first rows of effusion cooling, due to the low coolant superposition, the metal temperature is higher, leading to an increase of the heat sink. Fig. 4.9 highlights the comparison between the application of an averaged value and the specific value of each row. It is possible to notice that, as expected, the main effect is a sort of rotation of the curve that improves significantly the agreement with experimental data.

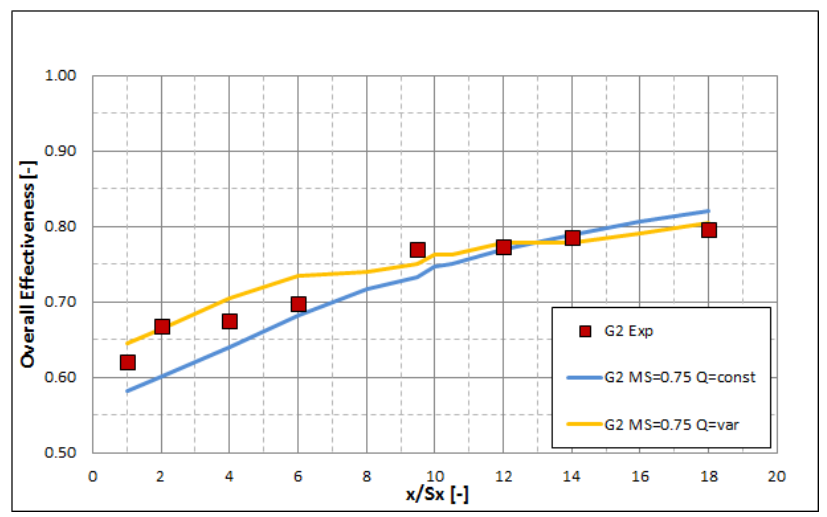

Figure 4.9: Effect of heat sink value (G2, $M=5$ )

\subsubsection{Sensitivity to heat sink discretization}

Further tests have been focused on the application of the heat sink on multiple source points. The discretization on one and three heat sinks have been assessed (see Fig. 4.10, but no appreciable differences have been observed. 


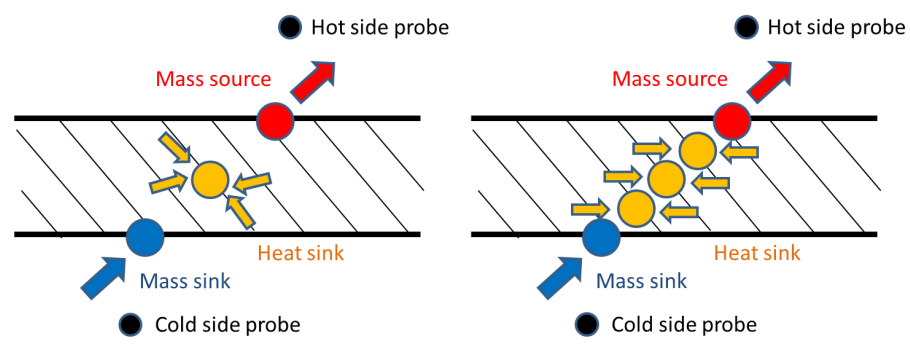

Figure 4.10: Heat removal discretisation on one (left) and three (right) heat sinks

\subsubsection{Sensitivity to blowing ratio}

In order to evaluate the validity of the procedure in different operative conditions, it has been decided to assess the model sensitivity to blowing ratio in range of $1.5-5$, which is typical of combustors. The results (depicted in Fig. 4.11) show a good agreement for $M=3$, whereas at lower blowing ratio $(M=1.5)$ overall effectiveness is overestimated, especially in the downstream part of the plate. This phenomenon can be explained comparing the flow field due to discrete holes with that one in presence of source points (see Fig. 4.12. It is possible to notice that, notwithstanding the velocity components have been imposed starting from the postprocessing of the previous simulation, the resulting injection angle is quite different and consequently the coolant protection on the plate. This effect can be ascribed to the application of the source point within the prism layer, close to the wall, which seems to make the injection angle almost orthogonal. Therefore, it may reasonably be supposed that this phenomenon has a greater impact as the blowing ratio decreases, since the mass sources are unable to model attached jets. As reported by Voigt et al. [112, this issue is strongly dependent on the distance from the wall, therefore further work will be necessary to enhance the accuracy of coolant injection. 


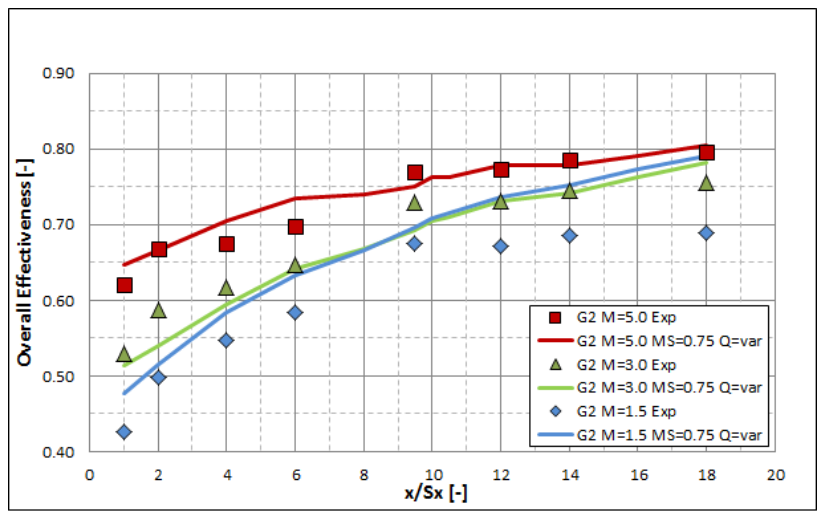

Figure 4.11: Effect of blowing ratio (G2, $M=5,3,1.5)$

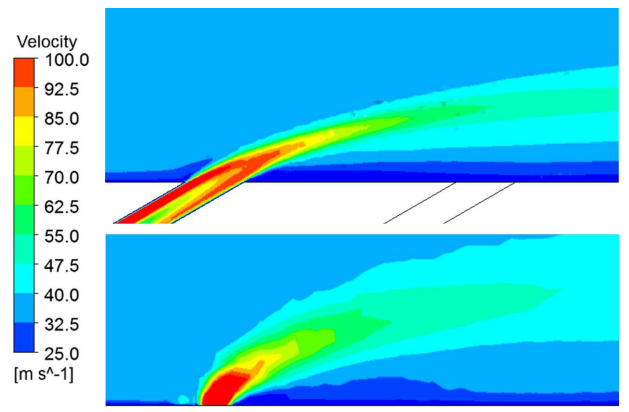

Figure 4.12: Comparison of the flowfield in presence of discrete holes (up) and source points (down) (G2, $1^{\text {st }}$ row, $M=5$ )

\subsubsection{Results: Pressure drop based formulation}

The best practices identified for an optimal use of the methodology are reported below. It is worth remembering that some guidelines have been established for hybrid meshing (tetra+prisms) and, in particular, for ICEM-CFD.

- Wall mesh elements should have maximum dimension equals to 
half of hole diameter (Max Size $=0.5 D_{h}$, in the particular case of ICEM-CFD);

- Volume mesh elements in the mixing zone should be refined enough to limit the numerical spreading of jets (Tetra Size Ratio $=1.2$, in the particular case of ICEM-CFD);

- Each hole should be modeled with its own value of heat sink (and presumably of mass flow rate);

- A single source point is sufficient to model the heat sink;

- The accuracy of the methodology is good at $M=5$ but decrease at lower values of $\mathrm{M}$. This phenomenon can be ascribed to the application of the mass sources at wall.

Subsequently, it has been decided to implement the local pressure drop dependent formulation, taking advantage of previous mentioned guidelines. Compared to the case with prescribed values to source points, the calculation of mass flow rate is carried out imposing a value of $C_{D}$ (in this particular case the experimental one, ranging from 0.63 to 0.72 , depending on diameter and blowing ratio), whereas the value of $h$ inside the holes is calculated starting from Cho and Goldstein correlation for short length holes 114.

In Fig. 4.13 is reported the final comparison between experimental data and the source-based methodology. It is possible to notice how the local pressure drop based formulation gives very similar results with respect to the case with prescribed values, having due advantaged of a limited number of inputs required. On the other hand, this approach implies a strong dependence on the accuracy of the way the inputs ( $h$ and $\left.C_{D}\right)$ are estimated. The same conclusions can be drawn from Fig. 4.14 where the same comparison has been carried out varying the blowing ratio.

Regarding the accuracy in the prediction of the mass flow rate, a final comparison has been made between numerical and experimental 


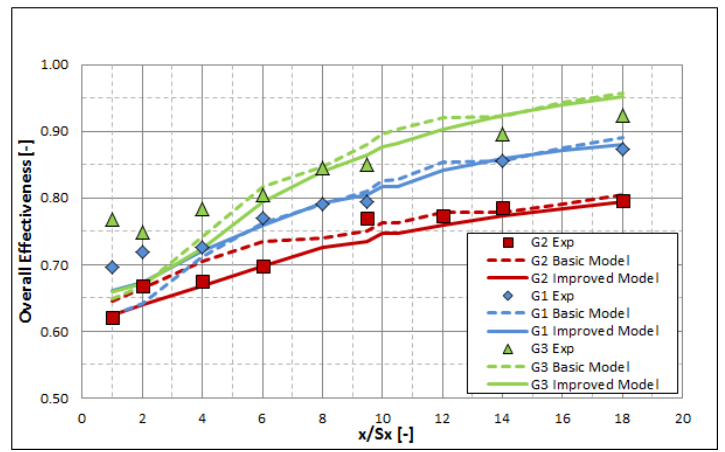

Figure 4.13: Final comparison between experimental data, source model with prescribed values and pressure drop formulation: effect of geometry $(M=5)$

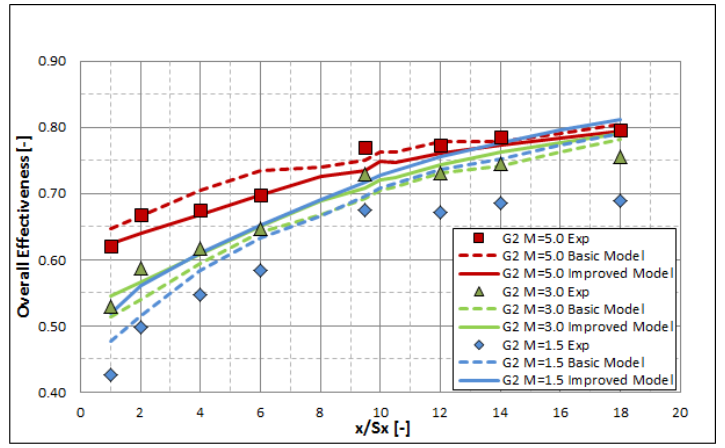

Figure 4.14: Final comparison between experimental data, source model with prescribed values and pressure drop formulation: effect of blowing ratio (G2)

data. Tab. 4.4 points out that, on varying the operating conditions ( $M=1.5,3,5)$ the relative error is smaller than $4 \%$. 


\begin{tabular}{cccc}
\hline $\mathbf{M}$ & $m \exp [\mathrm{g} / \mathrm{s}]$ & $m C F D[\mathrm{~g} / \mathrm{s}]$ & Relative error $[\%]$ \\
\hline 1.5 & 14.1 & 14.6 & 3.8 \\
3.0 & 26.9 & 27.4 & 2.0 \\
5.0 & 29.6 & 30.1 & 1.6 \\
\hline
\end{tabular}

Table 4.4: Comparison between experimental and predicted mass flow rate varying the blowing ratio $(G 2, M=1.5,3,5))$

\subsection{Model application: TECC-AE combustor}

Once checked the proposed methodology for accuracy, the model has been applied to an actual combustor in order to evaluate the feasibility of the methodology on an industrial case.

\subsubsection{Description of the test case}

The test case consist in a tubular combustion chamber developed by GE Avio in the context of an experimental campaign aiming at investigate a new injection system, characterized by a co-rotating pressure swirler. The dome is cooled by means of impingement jets, whereas the liner is protected by a ribbed heat shield and about 70 staggered rows of effusion cooling holes. The test point investigated consists of low-pressure conditions in proximity of lean blow out. Experimental data consist of pressure measurements along the flowpath and thermocouple temperature measurements on dome and liner. For each stream-wise position, three thermocouples were placed at different span-wise positions.

\subsubsection{Numerical setup}

The numerical setup is roughly unchanged with respect to the previous validation activity. Mass flow rate and total temperature have been imposed at the inlet, static pressure at bleeds and main outlet. In order to reduce the computational effort, it has been decided to take advantage of the periodicity condition of the combustor. Notwithstanding the possibility to simulate only $1 / 16^{t h}$ of liner and dome, the minimum 


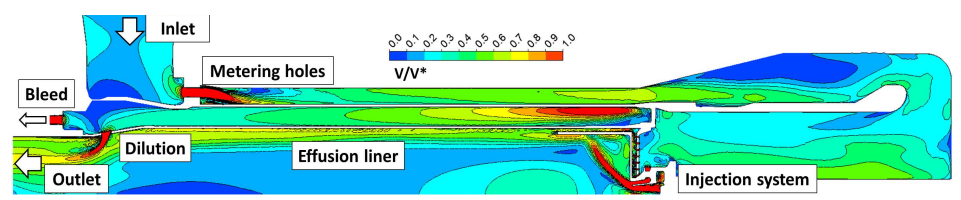

Figure 4.15: Flow field in the combustor meridional plane (without radiation)

periodicity angle of the swirler is 90 degrees, therefore it has been decided to couple the two parts with a mixing plane interface (which is named Stage in CFX). The grids of the fluid domains (19.8 million of elements) have been coupled to those of solid domains (1.5 million of elements) in order to perform a conjugate heat transfer (CHT) simulation. The fuel injection, which has been managed with a eulerian-lagrangian coupling and the TAB model for the particle breakup, has been modelled as a ring cone in the proximity of the injector lip in terms of cone angle, velocity temperature and mean diameter. Combustion is treated with Finite Rate Chemistry and Eddy Dissipation model, whereas radiative heat transfer has been computed with P1 model with a weighted sum of gray gases. For further details please refer to Andreini et al. [118].

\subsubsection{Results}

The following results have been normalized by reference values due to obligation of confidentiality. The velocity field is depicted in Fig. 4.15. It is possible to observe an overview of the combustor geometry and to notice the accelerations across metering holes, swirler, bleeds and dilution holes, as well as nearby the hot gas side of the liner, due to coolant injection.

A more detailed view is reported in Fig. 4.16. which focuses on the primary zone of the flame tube. The visualization by means of velocity vectors permits to show the flow field, highlighting the main recirculation zones, such as that one caused by the swirled annular jet and a less extended one in the corner of the dome. It also possible to notice geometric features as the impingement drilling and the ribbed heat shield, as well 
as the injection of coolant due to source points.

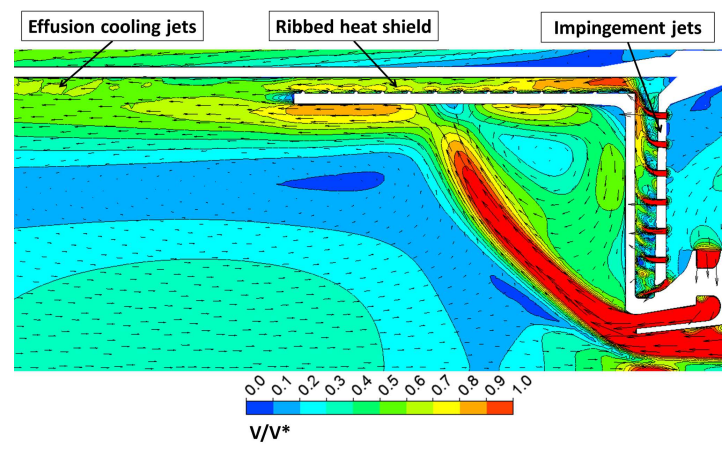

Figure 4.16: Flow field in the combustor meridional plane: detail of the primary zone with velocity vectors (without radiation)

A very interesting results of the present methodology is represented by the predicted values of discharge coefficients, which are dependent on the local flow conditions and, clearly, on the implemented correlation. The output has been plotted in Fig. 4.17, where the data have been averaged on each row in order to point out the stream-wise variations. This result has a direct effect on the global flow split of the component, which has been reported in Fig. 4.18 and hence on the distribution of air to the mixing process with fuel as well as to the cooling of the liner.

Hot gas recirculation in the corner of the heat shield is clear looking at the temperature field (see Fig. 4.19p. This phenomenon leads to a relatively hot zone in the proximity of the dome, confining the flame more downstream. It is interesting to notice the presence of a cooler zone within the swirled annular jet, which can be justified by the heat sink effect due to fuel evaporation. Furthermore, the structure of the flow field causes a sort of impingement of such annular jet on the heat shield, leading to the peak of temperature that can be seen in Fig. 4.20 where the temperature contours on both liner and heat shield are reported.

The comparison between experimental data and numerical results has 


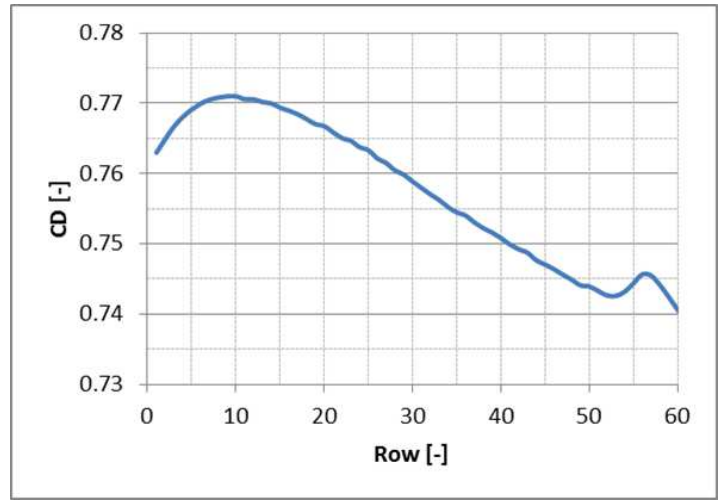

Figure 4.1\%: Stream-wise distribution of predicted discharge coefficients

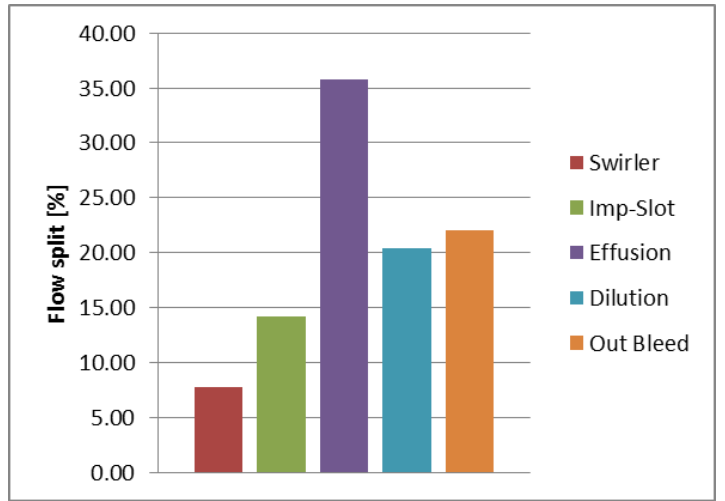

Figure 4.18: Predicted flow split within the combustor

been depicted in Fig. 4.21. The temperature profiles have been obtained by a circumferential averaging of metal temperature on the hot side. A good agreement can be observed along the liner, whereas a huge overprediction occurs along the heat shield, that worsens when radiation is taken into account. The temperature profiles remain roughly unchanged downstream, since flame is confined in the primary zone. The results 


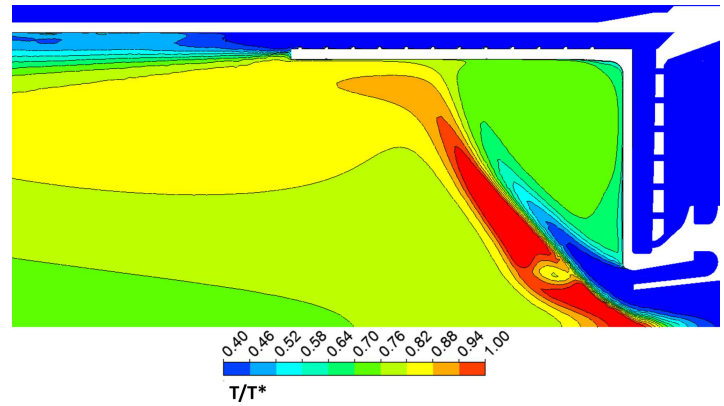

Figure 4.19: Temperature field of the combustor (without radiation)

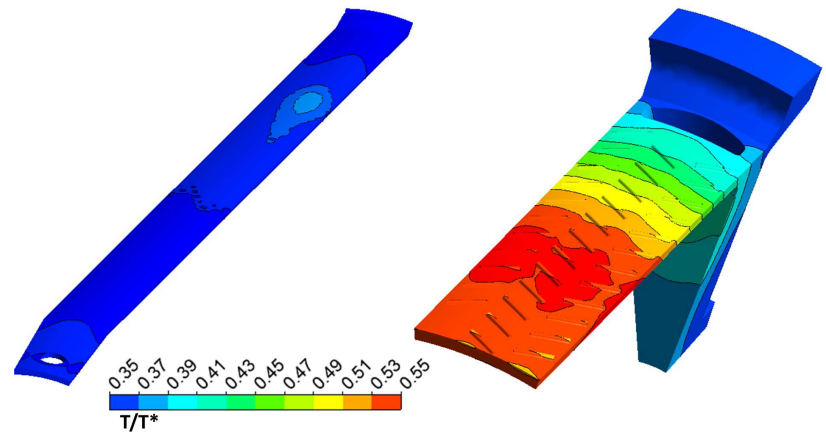

Figure 4.20: Temperature contour on liner and heat shield (without radiation)

obtained can be indicative of a poor prediction of the temperature field in primary zone, that anyway can not be attributed to the effusion cooling modelling.

\subsubsection{Conclusions}

A methodology for the modelling of effusion cooling holes has been presented. The main concept consists of replacing each hole with physical 


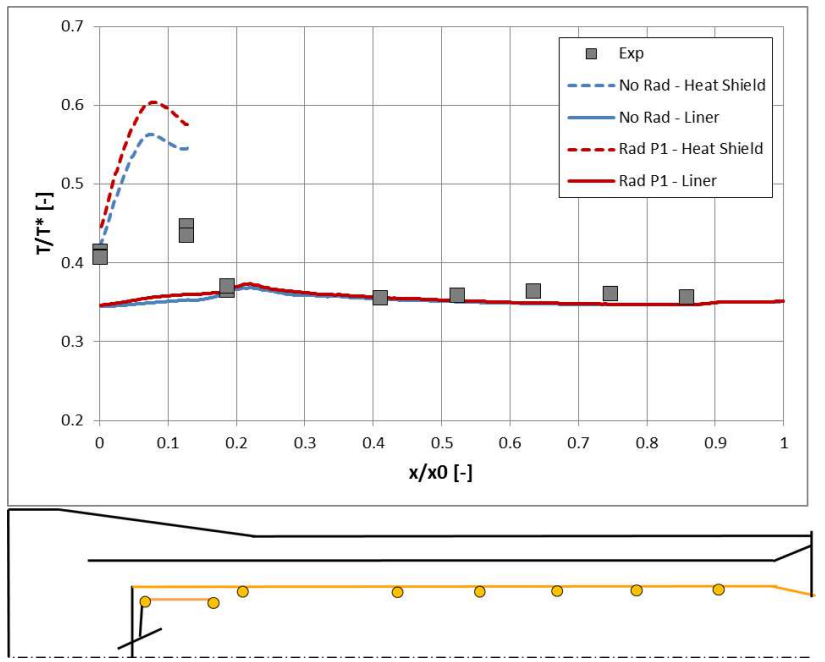

Figure 4.21: Temperature profiles along heat shield and liner

quantity sources and sinks to model both fluid aspiration and injection, as well as the removal of thermal power due to convection within the hole. The innovative aspect of this work in represented by the implementation of a local pressure drop based formulation for the calculation of mass flow rate, whereas discharge and heat transfer coefficients are calculated through a correlative approach.

A comprehensive assessment has been carried out to investigate the main parameters of interest and determine the best practices for a successful use of the procedure. In particular, a strong sensitivity has been observed for the mesh refinement. A comparison with a data set of overall effectiveness, deriving from an experimental campaign aimed at investigating the thermal behaviour of effusion liners, has showed a good agreement, which diminishes with the decreasing values of blowing ratio, due to the application of mass sources at wall.

Subsequently, the methodology has been applied on an actual combustor test rig. Notwithstanding the uncertainties due to the numerical setup 
(turbulence, combustion, particle breakup, radiation, effusion modelling by means of sources) a good accuracy has been showed, at least where source points are applied.

The present work represents a first step in the validation of the proposed approach, which aims at becoming a reliable methodology in both design and analysis phases of such components. Notwithstanding the difficulties due to the high memory requirements having reference to the number of source points, the procedure has positioned itself as an effective option compared with both uniform effusion injection (which does not permit an accurate evaluation of heat transfer) and discrete hole meshing (that leads to a prohibitive computational cost). Additional efforts will be addressed to investigating the effect of mesh refinement and mass source distance from wall on adiabatic effectiveness and heat transfer coefficient augmentation due to injection, as well as the application of the methodology to different contexts, such as impingement cooling.

\subsection{Model application: NEWAC combustor}

This section describes the main findings of a numerical activity aiming at simulating a lean burn annular combustor cooled by means of effusion. In order to reduce the computational effort, the source based model developed for the effusion cooling has been exploited. In the context of this activity, the attention has been focused on the prediction of pressure losses and flow split, as well as the assessment of the metal temperature. A comparison has been carried out making use of the temperature measured during an experimental campaign on a dedicated combustion test rig. Furthermore, the results have been compared to different methodologies applied for the preliminary design in the industrial field. In particular, in the context of GE Avio, the preliminary design of combustors involves numerical simulations carried out with thermal flownetwork solvers commercial (Flowmaster) and in-house codes (Therm1D). 


\subsubsection{Description of the test case}

The test case consists in the prototype of single annular combustor developed in the context of the NEWAC European project (see Fig. 4.22). This combustion system, based on lean burn combustion, achieves low $\mathrm{NO}_{x}$ emission using an innovative injection system called PERM (Partial Evaporation and Rapid Mixing), developed for medium overall pressure ratios $(20<O P R<35)$. The device, whose sketch is reported in Fig. 4.23 is characterized by co-rotating double radial swirlers. Flame stabilization is achieved through fuel staging between the pilot injection from a pressure atomizer (for low-power stability) and the main injection achieved through the formation of a film on the inner side of the lip.

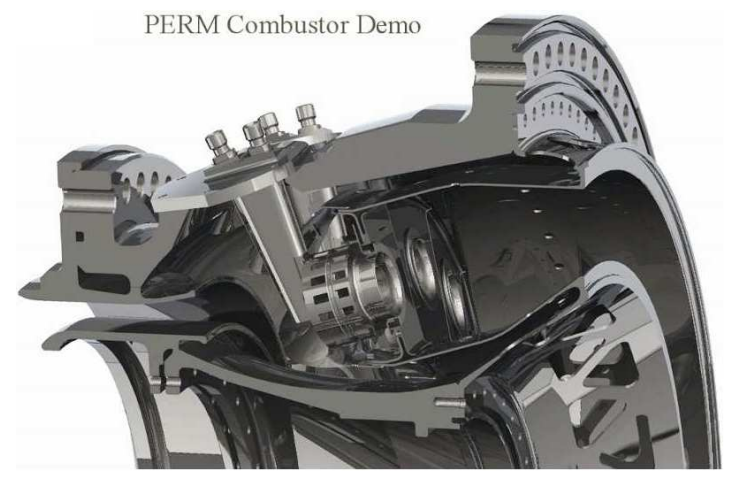

Figure 4.22: GE Avio's NEWAC combustor prototype

As far as the combustor is concerned, part of the air passing through the dump diffuser is directed to the annuli where it is used for the liner cooling, the dilution holes and the bleeding holes. The remaining part is instead divided between the injection system and the dome cooling. The dome is protected by an impingement cooled heat shield, which additionally provides slot cooling through coolant discharge, whereas the liners are equipped with staggered arrays of inclined effusion cooling holes with circular cross section. 


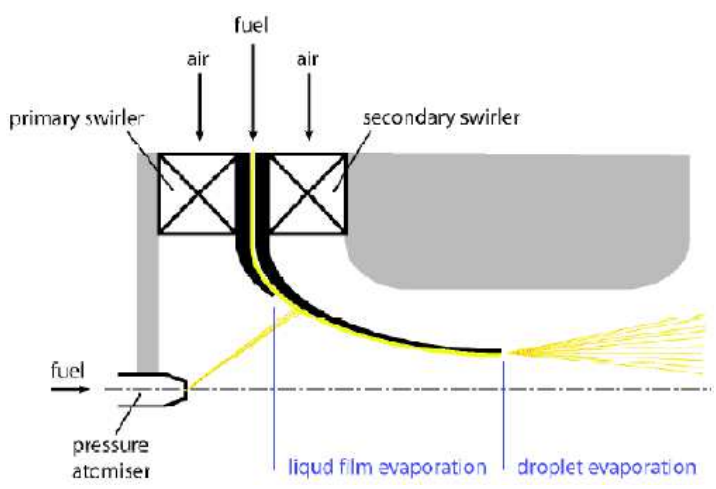

Figure 4.23: Sketch of the injection system

The details about the operating point considered in this work have been reported in Tab. 4.5 As far as the experimental campaign is concerned, available data consist of pressure measurements in proximity of the prediffuser discharge, the annuli entrances and the spark location. Metal temperature was measured by means of thermocouples instrumented on both inner and outer liner at different axial and circumferential position.

\begin{tabular}{cc}
\hline \hline Operating conditions & \\
\hline Inlet $P_{t}[\mathrm{bar}]$ & 29.4 \\
Inlet $T_{t}[\mathrm{~K}]$ & 794 \\
$\dot{m}_{\text {air }}[\mathrm{kg} \mathrm{s}-1]$ & 42.0 \\
Outlet $P[\mathrm{bar}]$ & 28.0 \\
$\dot{m}_{\text {fuel }}[\mathrm{kg} \mathrm{s}-1]$ & 0.90 \\
$\dot{m}_{\text {fuel pilot }} / \dot{m}_{\text {fuel }}[-]$ & 0.05 \\
\hline \hline
\end{tabular}

Table 4.5: Description of the investigated test point

\subsubsection{Numerical setup}

3D CFD RANS conjugate calculations have been considered in this study, referring to the Navier-Stokes equations solver ANSYS ${ }^{\circledR}$ CFX 
v14. Compressibility effects have been taken into account and a High Resolution advection scheme has been used, which automatically blends between first and second order scheme throughout the domain based on the local solution field 113 . The fluid has been modelled as a reacting mixture, whereas thermal conductivity and emissivity were specified for the metal properties. Energy equation has been solved in terms of total energy and viscous heating effects have been accounted for.

Due to the presence of strong recirculating regions and detached flows, turbulence modelling plays a central role in the simulations of combustion chambers. Even though the k- $\omega$ SST model would represent the most appropriate choice due to the possibility of exploiting a Low Reynolds near wall treatment, in the context of this activity the $\mathrm{k}-\varepsilon$ model has been employed. A preliminary assessment of the turbulence models on this type of injector by means of PIV measurements has in fact highlighted a better agreement, whereas the k- $\omega$ SST model returns a greater intensity of the recirculating zone, which leads to a non-physical (and not confirmed by experiments) ignition within the injection system. These considerations have been confirmed also by Kern et al. [27, which investigated the flow field of a similar injection system by means of PIV and RANS simulations. As far as the near-wall treatment is concerned, the scalable wall function approach made available by the code has been exploited, which overcomes the issues related to the application of standard wall function on grids with $y^{+}<11$, hence reducing the sensitivity to local mesh refinements.

The considered fuel is JetA. A conventional eulerian-lagrangian discrete particle model was employed to track JetA liquid fuel. Fuel is injected directly on the pilot whereas the atomization of the fuel film is modelled through the injection at the end of the lip (see Fig. 4.23. Second particle break-up has been modelled using the Taylor Analogy Breakup (TAB) theory. Combustion is treated with the combined Finite Rate Chemistry/Eddy Dissipation model, which computes the reaction rates for each model separately and then uses the minimum of the two, and modelled by means of a two-step kinetic scheme. Both turbulent Prandtl and Schmidt numbers have been set to 0.9 , so as to obtain a Lewis 
number equal to 1 , with the aim of fully respecting the mass-energy transfer analogy. Radiative heat transfer has not been accounted for at the moment.

A sketch of the computational domain is reported in Fig. 4.24 It is worth noticing that, compared to the engine configuration reported in Fig. 4.22 in the experimental test rig the annuli are connected to a plenum where the hot gas is discharged and the bleeding mass flow rate is controlled by means of metering holes. The inlet boundary conditions have been assigned in terms of mass flow rate, total temperature and turbulence intensity, whereas an iterative expression as been implemented to adjust the static pressure at the outlet so as to match the experimental value measured within the combustion chamber. In order to reduce the computational effort, the domain has been limited to a $20^{\circ}$ sector of the single annular combustor and a periodic condition has been imposed. Fluid and solid domain have been interfaced through General Grid Interface (GGI) across heat shield and liners in order to perform a Conjugate heat transfer (CHT) simulation, whereas adiabatic conditions have been applied to all other surfaces. The fuel injection has been characterized by means of temperature, direction and velocity magnitude of the particles, while a Rosin-Rammler distribution was considered for the droplet size.

The perforations of both liners have not been explicitly reproduced, taking advantage the developed effusion cooling model. Each hole has been replaced by three source points (a mass source, a mass sink and a heat sink), calculating the value of $C_{D}$ and $h$ through the above mentioned correlations. Furthermore, also the the dome perforation has been modelled by means of source points, even though heat sink has not been taken into account in this case. With this modelling strategy, over 5000 source points have been applied in the simulation. In order to track the spatial distribution of the coolant, an additional transport equation was added for a passive scalar Coolant representing coolant mass flow, whose values are 1 for the flow coming from the source points and 0 at the inlets of the domain.

The software ANSYS ${ }^{\circledR}$ ICEM-CFD has been used to generate the 


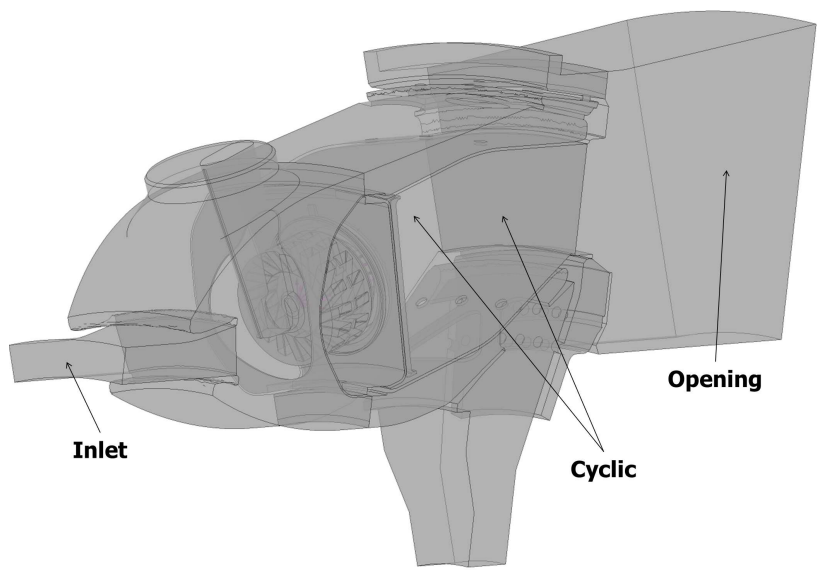

Figure 4.24: Sketch of the computational domain and assigned boundary conditions

computational grids, realized with an increasing refinement level in order to perform an assessment of the mesh sensitivity (see Tab. 4.6). These grids were characterized by tetrahedrons with 10 layers of prisms close to the wall, whereas he solid domain mesh (tetrahedral for the heat shield, hexahedral for the liners) counts about 0.58 million elements. A mesh sensitivity analysis has been carried out in order to establish the adequate level of mesh refinement to capture the flow physics. The results highlighted a huge influence in correspondence of the injection system due to the effect of spatial discretization on droplets evaporation and the subsequent mixing between air and fuel. As a result, despite a roughly unchanged flow field, some differences in mixture fraction distributions and hence temperature field have been noticed. Despite these evidences, a further refinement have not been investigated, since different local peak values of temperature have proved to affect mainly $\mathrm{NO}_{x}$ emissions without relevant effects on the wall temperature.

The solution convergence has been assessed by monitoring the residuals, the imbalance across the domains and appropriate monitor points of 


\begin{tabular}{ccc}
\hline \hline Mesh & No. elements (10e6) & No. nodes (10e6) \\
\hline A & 20.1 & 6.9 \\
B & 41.7 & 14.3 \\
C & 58.8 & 17.2 \\
\hline \hline
\end{tabular}

Table 4.6: Grids generated for the fluid domain

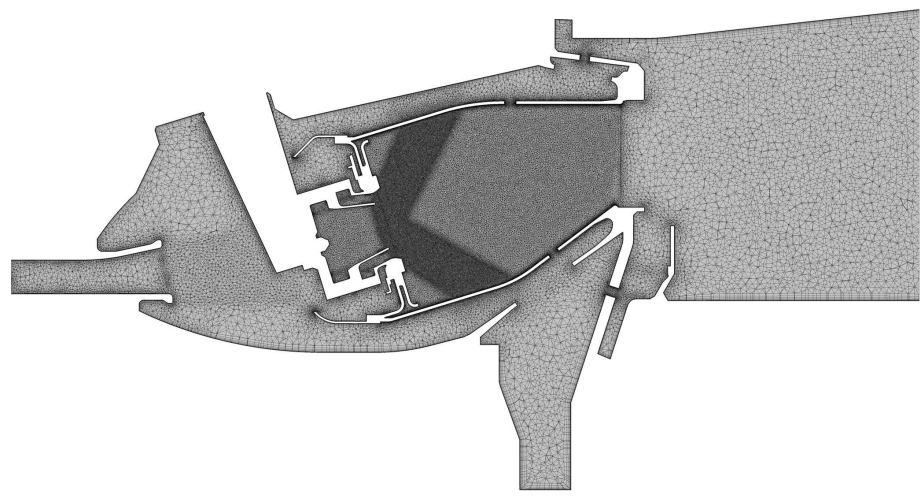

Figure 4.25: Computational mesh of the fluid domain (Mesh C)

pressure and temperature in representative locations. Simulations have been stopped when these trends reached a steady-state.

\subsubsection{Results}

\subsubsection{Flow field and pressure distribution}

One of the main targets in the design of a combustor is represented by the pressure drop across the device, since it involves a reduction in the efficiency of the entire machine. At the same time, it influences the flow split within the combustor, which plays a central role in the flow distribution and hence in the control of metal temperature and the flame stabilization. For these reasons, CFD is an essential tool for an accurate prediction of the flow field, especially considering that a 
correlative approach is unable to capture the three-dimensional nature of the diffuser and the injection system.

Fig. 4.26 shows the contours of velocity on the meridional plane passing through the injection system and highlights the main features of the flow field by means of vectors, which have been normalized for sake of readability. The combustion chamber is almost entirely occupied by the recirculation zone generated by the swirler, which extends up to the dilution holes, where the vortex breakdown takes place, drawing hot gas towards the injection system in order to stabilise the flame.

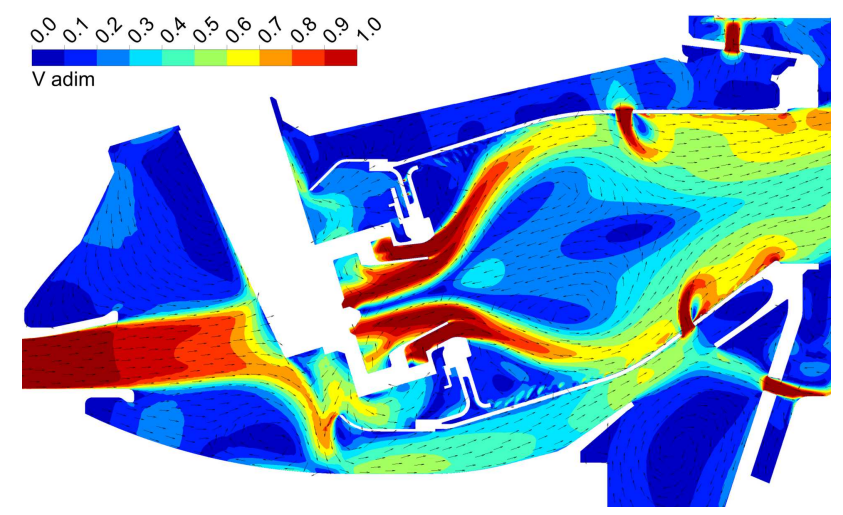

Figure 4.26: Flow field within the combustor

As far as the pressure losses are concerned, the values measured during the experimental campaign, those obtained by the flow-network solver Flowmaster and the results of the CFD simulation have been reported in Fig. $4.27\left(\mathrm{P}_{\text {ref }}\right.$ represents the value of total pressure at the exit of the pre-diffuser). It is worth pointing out the capability of both numerical approaches in predicting the pressure field within the combustor, even if only CFD takes into account the three-dimensional nature of the flow field. Nevertheless, a very good agreement has been achieved (with a relative error of about $+0.5 \%$ ). In the absence of experimental measurements of the flow split, this result can provide a reasonable confidence on the 
predicted flow distribution within the combustor.

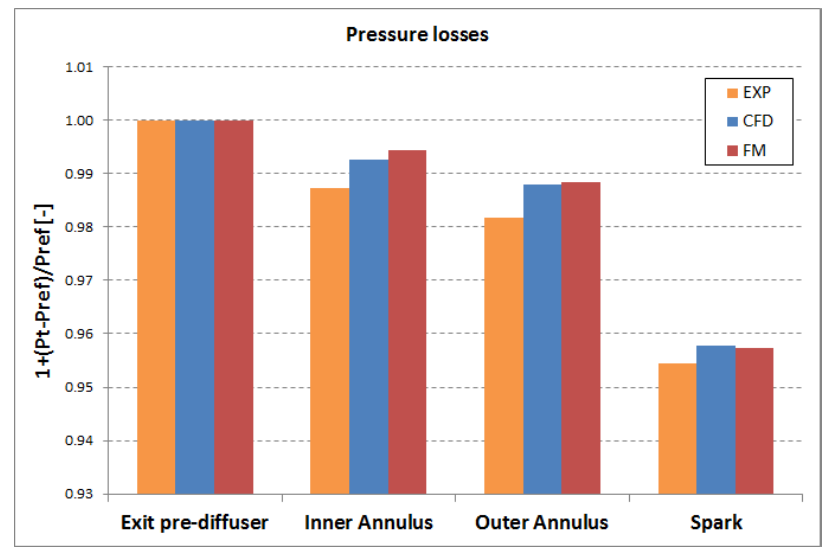

Figure 4.27: Comparison between experimental and predicted pressure losses

It is well known that the flow split represents a key parameter in the design of a combustor, since it influences both the amount of air dedicated to the injection system and the thermal management of the components. These considerations are even more critical in the case of lean burn combustors, due to the reduced amount of available coolant and the proximity of the flame to the blow-off condition. A comparison between CFD and flow-network solvers, based on a correlative approach, has shown a good agreement in estimating the flow split within the annuli, thus confirming the validity of the correlations used for the preliminary phases of the design.

\subsubsection{Temperature field}

The predicted temperature field is reported in Fig. 4.28. It is important to point out the main role of central recirculation zone in flame stabilization. Due to the very efficient liquid fuel atomization and the following rapid evaporation, a premixed like flame front is observed; it is 
located in the shear layer generated between the annular jet of the swirler and the main central recirculation. In such region a relevant mixing rate is induced by local high turbulence intensity while hot gases, provided by recirculating flow, assure the continuous ignition source. Relatively cold gases are observed to feed the toroidal external recirculation zones and therefore not relevant heat loads are expected on the heat shield installed on combustor dome as well as in the early part of inner and outer liner.

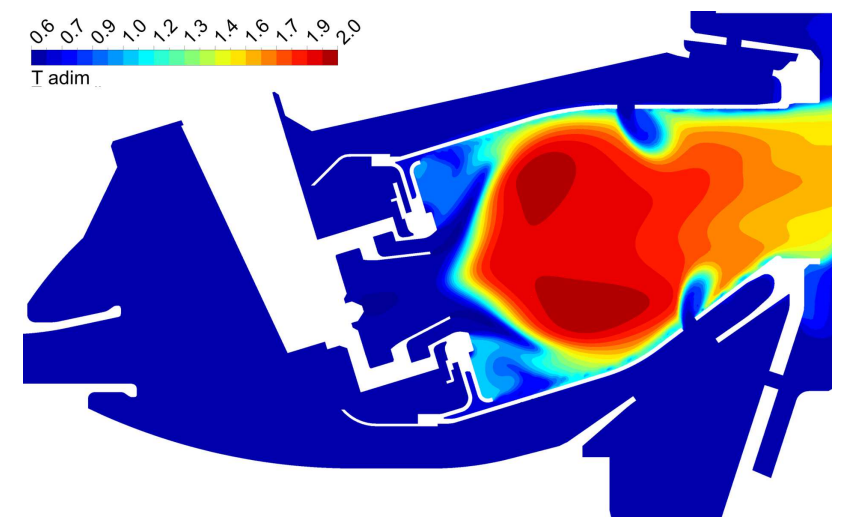

Figure 4.28: Temperature field within the combustor

An overall contour map of computed metal temperature is reported in Fig. 4.29. In order to assess the robustness and reliability of the proposed CFD methodology, computed metal temperature are compared with data obtained by thermocouples installed along the liner and with the preliminary design predictions by in-house Therm1D methodology. It is worth pointing out that some thermocouples are located also at different circumferential positions, so as to make possible the evaluation of the temperature variation in circumferential direction.

Profiles of metal temperature on the hot side of the liners extracted from CFD results are reported in Figs. 4.30 and 4.31. The values are normalized due to confidentiality issues and are expressed in terms of $T_{\text {adim }}=T / T_{\text {ref }}$ as a function of a non-dimensional curvilinear abscissa 


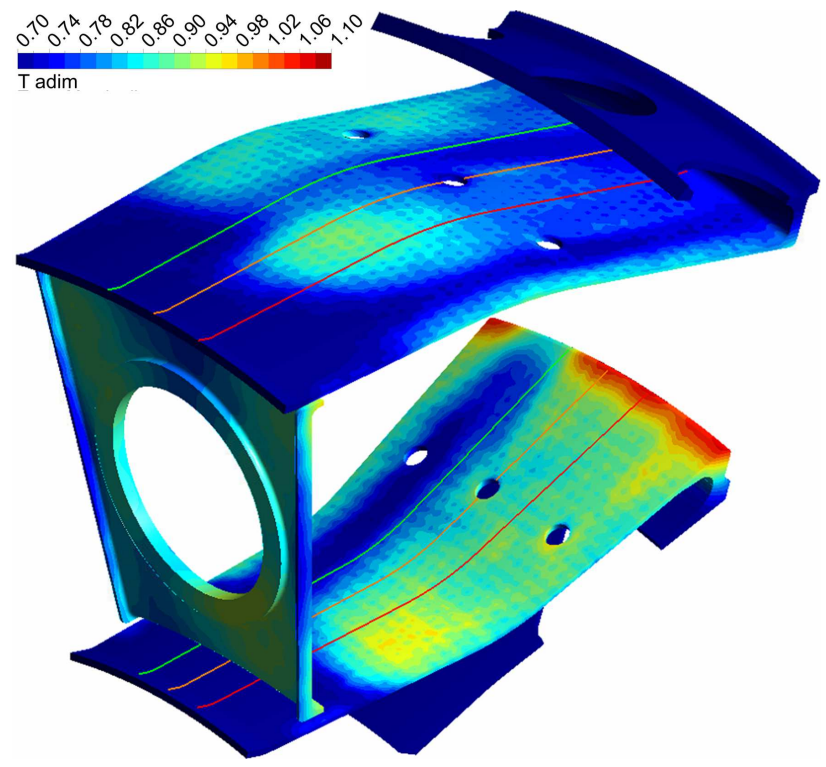

Figure 4.29: Contour of metal temperature

$S / S_{\text {tot }}$. In order to have a more consistent comparison among the data sets, four different profiles have been extracted by CFD results. The following profiles are considered:

- INJ refers to the exact tangential location of thermocouples.

- INJ $\pm 1 / 2$ refers to the previous location \pm half tangential pitch of dilution holes.

- AVERAGE refers to tangential averaged metal temperature axial distribution.

As confirmed by the contour map reported in Fig. 4.29, a strong variation of metal temperature is observed in tangential direction. This phenomenon makes the comparison among CFD results and discrete thermocouples as well as intrinsic tangential averaged results of 1D method- 
ology a complex task. Nevertheless is important to point out how CFD results reasonably match the trend of experimental data along the entire extent of the component, with an overall acceptable agreement at least on the outer liner. It is in fact necessary to take into account the uncertainty related to metal temperature measurements in this type of full annular tests, which is not only related to the accuracy of the thermocouples but also to possible mismatches in their location with respect to the injection system and therefore the flame.

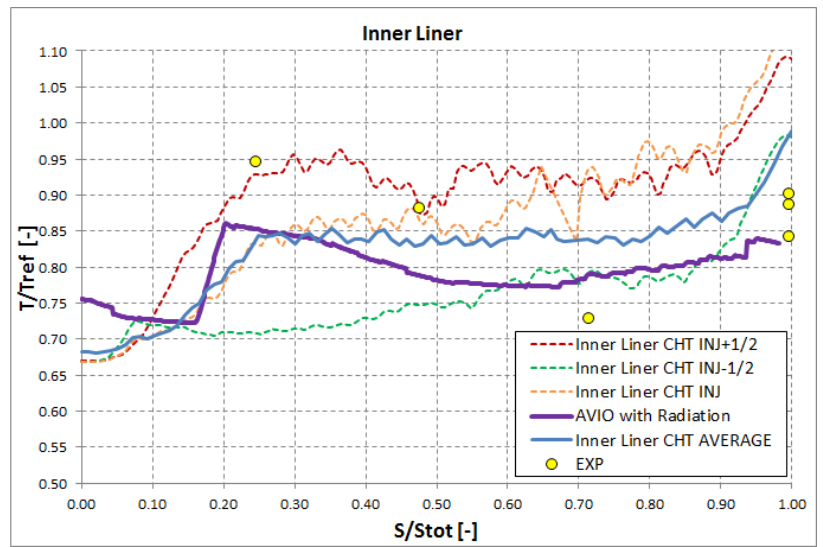

Figure 4.30: Comparison between experimental and predicted values of metal temperature

On the prediction side, it is important to recall that metal temperature can be considered the final outcome derived from a complex interaction among different phenomena such as radiative heat loads, internal heat sink within the effusion holes and both internal and external convective loads (hot and cold gas side, respectively).

One of the most challenging aspects in combustor metal prediction is indeed represented by the estimate of the internal convective heat loads, since the heat transfer coefficient distribution is deeply influenced by the interaction between film cooling jets, which causes a significant heat 


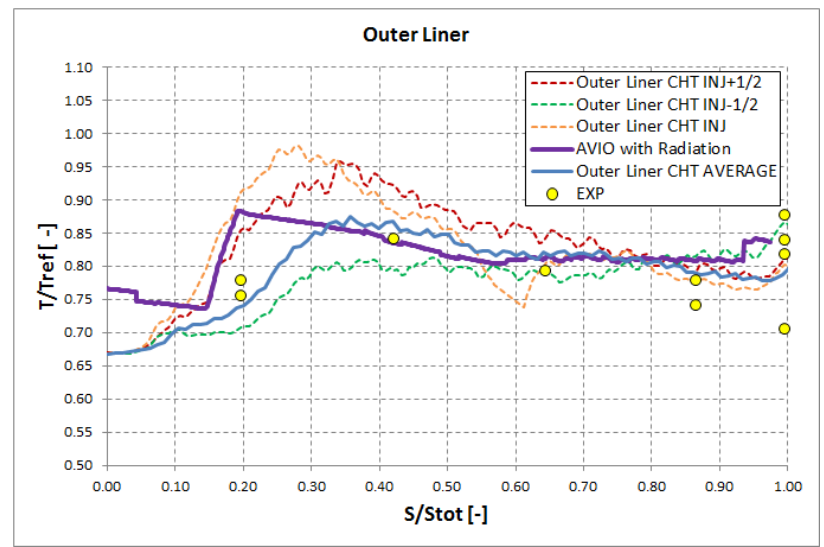

Figure 4.31: Comparison between experimental and predicted values of metal temperature

transfer augmentation, whereas the coolant protection, affected by mixing and superposition phenomena, leads to significant reduction in the driving gas temperature.

In order to better explain the resulting metal temperature field returned by CFD, and in particular the significant variation along tangential direction, the near-wall coolant distribution has been reported plotting the contour of the transported passive scalar Coolant (see Fig. 4.32). The coolant protection is maximum in the very first part of the liners, where the contribution of the cold flow discharged from the slots provides a significant reduction of the fluid temperature and hence limiting the convective loads. Immediately downstream of the stagnation region produced by annular jet, a strong interaction with the swirled flow occurs, leading to a relevant reduction in the coolant protection associated to the peak in metal temperature (as confirmed by Patil et al. [61]). Most of the coolant coming from the upstream rows and slots is in fact entrapped by a sort of blockage effect that confines part of the cooling flow in the dome recirculation regions (see also Fig. 4.33). This phenomenon seems 
confirmed by experimental tests carried out on a three-sector rig in terms of PIV and heat transfer measurements (for further details please refer to Andreini et al. 119]). As a secondary effect, the coolant is locally redirected, producing non uniform accumulations. All these phenomena have a direct impact on the metal temperature, as it is possible to notice comparing Figs. 4.29 and 4.32 As far as the last part of the liners is concerned, the effect reported by Martiny et al. [40] characterised by coolant lift off due to dilution jets is highlighted, whereas the effect of superposition leads to a progressive increase in the Coolant concentration. Nevertheless, the profiles of metal temperature depicted in Figs. 4.30 and 4.31 show a roughly constant trend, which, bearing in mind that radiation in not accounted for and assuming little variations in the heat loads on the cold side and within the cooling holes, can be ascribed to an increase in the hot side heat transfer coefficient in stream-wise direction due to the acceleration of the gas approaching the exit of the combustor.

With the aim of further investigating the above mentioned coolant accumulation, two additional contours of Coolant have been plotted on cross-planes respectively at $1 / 3$ and $2 / 3$ of the distance between injection system and dilution holes (white lines in Fig. 4.33). In Fig. 4.34 the normalized vectors clearly highlight the flow field outside of the central recirculation zone: the coolant injected is convected towards the corners of the combustion chambers, where secondary flows occur due to the interaction with the adjacent sectors.

The discussed strong non uniform distribution of effusion cooling air is certainly the most critical issue in the CFD results. Despite the described proper physical effect justifying coolant accumulation, computed results are predicting a significant underestimation of coolant to gas mixing, that can be ascribed to modelling issues. RANS eddy viscosity turbulence models are known to under-predict the mixing of jets in cross flow. This limitation is mainly due to steady state and isotropic assumption of the turbulence field. In typical industrial RANS calculations the common way to mitigate this limitation is to increase turbulence mass and thermal diffusivity reducing the turbulent Prandtl and Schmidt numbers. Several 


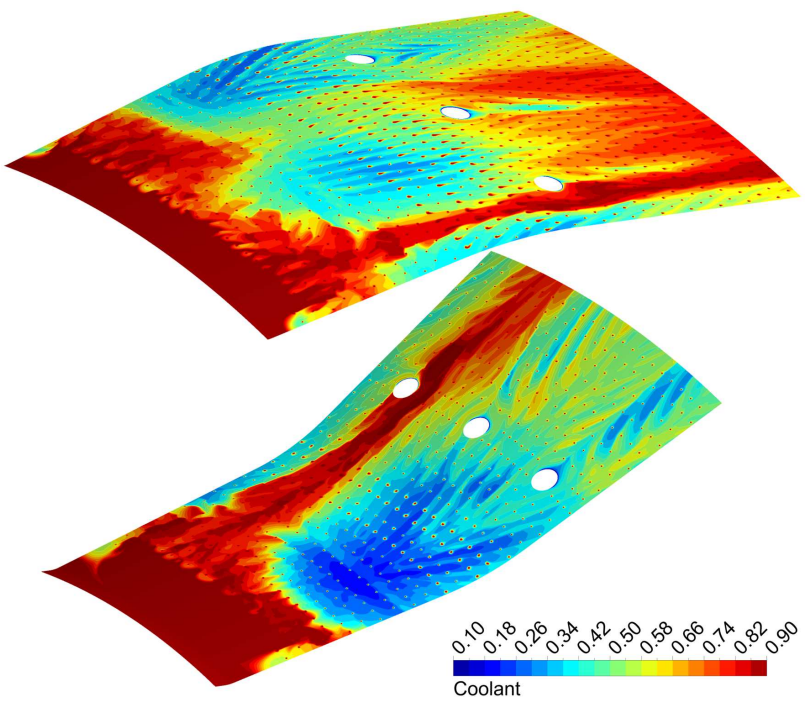

Figure 4.32: Contour of transported passive scalar Coolant

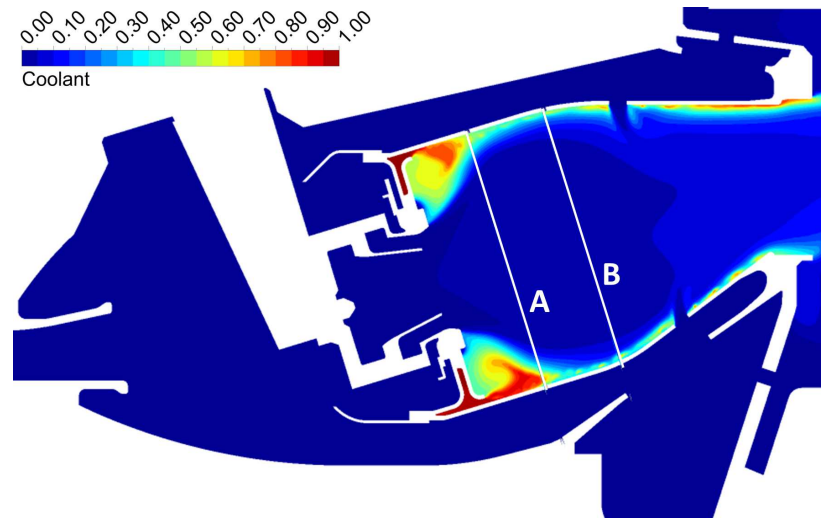

Figure 4.33: Coolant field within the combustor 


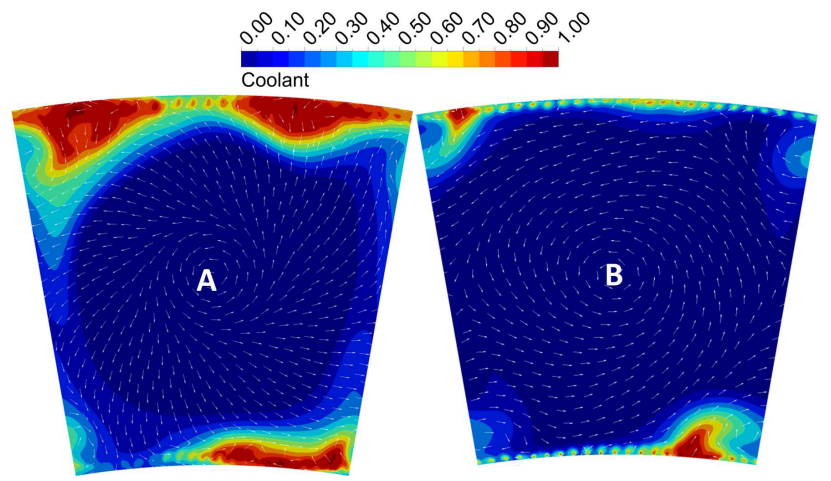

Figure 4.34: Coolant distributions at increasing distance from the injection systems

works report successful applications of this approach [120, 121, but very few of them deal with conjugate heat transfer computations. When metal temperature prediction are to be carried out, in fact, the modification of turbulent Prandtl number, required to match cooling air mixing, may have a significant effect also on wall heat flux, with a non acceptable increase in heat transfer rate. It is therefore necessary to properly evaluate the twofold effect of turbulent Prandtl number tuning on mixing and heat transfer that may require the introduction of wall-distance dependent thermal diffusivity [77. Due to this last discussion, this first application of the proposed local source methodology for CFD analysis of effusion cooled combustors was carried out by keeping the standard 0.9 turbulent Prandtl/Schmidt number, leaving the introduction of a specific set of validated design practices regarding turbulent Prandtl/Schmidt number tuning to future developments.

\subsubsection{Conclusions}

In the present activity the results of the application of a methodology for modelling of effusion cooling are presented. This approach consists in replacing the fluid extraction/injection of each hole by means of mass 
sink/source, whereas the heat pick-up within the hole is taken into account through a heat sink. The calculation of mass flow rate and thermal power is computed at run time depending on the local fluid dynamic conditions and appropriate correlations.

The present CFD methodology has been compared with preliminary design tools based on thermal flow-networks and assessed making reference to an experimental data set obtained on a combustion test rig. The application to this specific test case has highlighted a very good agreement in predicting the pressure losses within the combustor, providing a good confidence on the resulting flow split. Even if the three-dimensional nature of the flow field is taken into account only by CFD, the flow distribution within the annuli predicted by flow-networks proves accurate and hence a feasible approach for a preliminary design.

Some discrepancies have been noticed as far as the metal temperature field is concerned. The comparison with the experimental data set has highlighted a good agreement for the outer liner, while the trend is quite mis-predicted on the inner liner. Investigating the metal temperature pattern, the evaluation of the transported passive scalar Coolant has pointed out a very non-uniform coolant distribution due to the interaction between the swirled flow and the effusion cooling system. The absence of any specific numerical treatment to fix typical jets in cross flow mixing under-prediction of steady RANS approach prevents the possibility to fully catch the smoothing effect due to turbulence mixing. A specific investigation is going to be carried out in this field representing the next future perspective of this activity.

The non-uniform prediction of film effectiveness on the liner is the main source of inaccuracy in metal temperature prediction, which needs to be added to thermocouples uncertainty in terms of measured data and actual installation position. Despite this first application of the proposed source points methodology has proved to be a fairly reliably way to have a full CFD predicted thermal behaviour of modern effusion cooled combustors. 


\subsection{Model application: LEMCOTEC}

Following the validation phase, the methodology has been successfully employed within the framework of LEMCOTEC project with the aim of identifying the best performing configuration amongst three different geometry. The investigation was focused on the estimation of several design target parameters, such as combustor pressure drop, flow split and temperature conditions at the outlet. This activity allowed to provide indications that led to the final design of the novel LEMCOTEC combustor prototype, whose manufacturing is on going and will be tested during 2015.

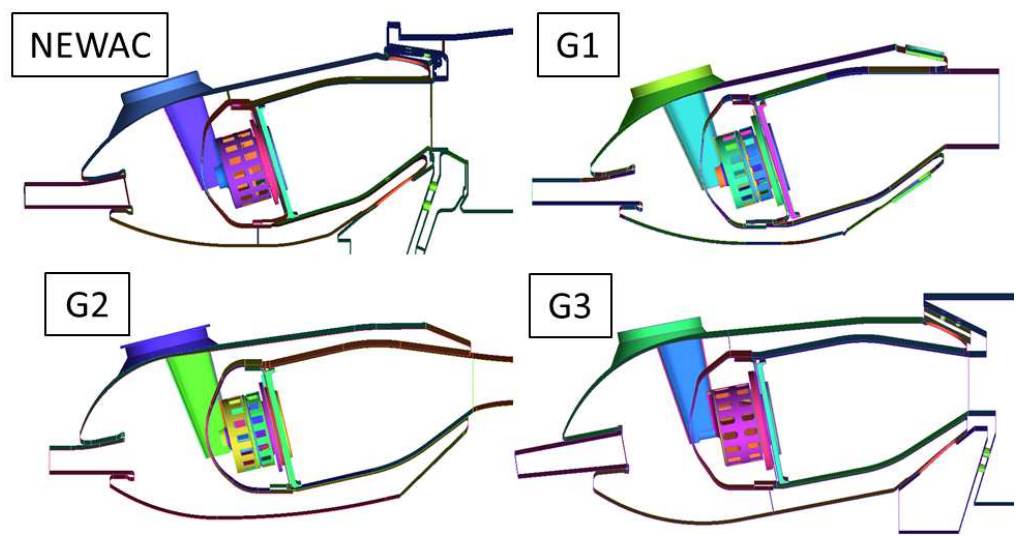

Figure 4.35: Configurations investigated in the framework of LEMCOTEC project

\subsection{Final remarks}

This chapter described the phases of development and validation of a model for effusion cooling holes. This approach allows the employment of relatively coarse grids with a mesh size of $0.5 D$ (2 elements for hole diameter) and the run time calculation of the mass flow rate depending 
on local fluid dynamic conditions, proving to be a viable methodology for the estimation of combustor pressure losses and flow split.

The injection of coolant within a single cell entails intrinsic limitations to the model, mainly ascribable to the erroneous jet trajectory, showing potential improvements in the estimation of thermal loads. Moreover, further modifications could concern the formulation made available within ANSYS CFX, that leads to a significant increase in computational effort when a huge number of source points is included. This issue could be fixed dropping out the standard implementation in place of a user defined one by means of User Fortran. 



\section{Chapter 5}

\section{Methodologies for thermal design of aero-engine combustors}

A reliable design tool for the thermal management of hot gas path components is vital to improve the capabilities and fulfil all the technological requirements demanded to a modern combustor. The development process of a new product is characterized by several steps, in which it evolves from a simple concept to its final configuration. The process could be thus subdivided in different phases: the concept design, the preliminary design and the detailed design, after which the actual performance of a prototype are usually verified by means of experimental tests.

Each phase is characterized by intermediate checks aimed at confirming if the targets are fulfilled and, in case, revising the design. Certainly, each iteration with the previous steps involves significant investments, that generally increase in the final design phases due to growing complexity of the analyses, i.e. computational cost and test rigs setup.

As a consequence, an appropriate trade-off between speed and accuracy must be identified during the development of the design tools; concept design usually consists in 0D analyses, whereas in the preliminary design the geometry is studied by means of equivalent 1D flow networks and simplified 2D CFD/FEM models, that exploit correlative approaches 
aimed at approximatively estimating pressure losses and heat transfer. This phases are characterized by the investigation of many geometries, often using optimisation codes to identify the most promising configurations able to provide the required performance. Afterwards, more detailed (and onerous) investigations are carried out through 3D CFD analyses, in order to verify the validity of the design choices before the manufacturing of a prototype.

This chapter describes the in-house 1D thermal methodology (Therm1D) currently used by University of Florence and GE Avio for the preliminary design of combustor cooling systems. The tool allows the estimation of flow split within the combustion chamber and liner wall temperature, through the solution of an equivalent flow network and a wide database of correlations. Subsequently, an innovative methodology (Therm3D) is presented, which is supposed to provide accurate data for the detailed design phase. This kind of investigations, usually performed through CHT CFD analyses, are barely affordable due to the implementation of effusion cooling. The proposed approach is based on the coupling between CFD and a flow network solver; one of the most peculiar features is represented by the source-based modelling of effusion cooling.

\subsection{Preliminary design: Therm1D}

\subsubsection{D thermal methodologies}

Notwithstanding the increasingly spread application of CFD within the industrial field, the employment of one-dimensional tools still plays a central role, especially during the preliminary design of complex systems such as gas turbines. They rely on simplified models that describe the different parts of the system, from simple orifices, straight and curved pipes to more complex models representing impingement plates, effusion cooled or double-wall liners. For each model, the one-dimensional Navier-Stokes equations, including dedicated correlations that describe the phenomena of pressure drop and heat exchange. To provide a solution of the whole system, the single models are coupled together giving rise to a fluid 
network of the cooling system, whereas appropriate boundary conditions must be provided to the metallic parts with the aim of calculating the temperature distribution of the investigated components.

The main advantage of these tools lies in the very short time needed to modify and simulate the system, making the most appropriate approach when a significant number of configurations must be tested, or their performance at different operating conditions. In such context the effort required for additional CFD simulations, with the related modification of the CAD and generation of a new mesh, would be unbearable. For this reason, they may be used in conjunction with optimization codes or to perform Design of Experiments aimed at pointing out the importance of operating parameters.

On the other hand, several limitations, intrinsic within the 1D approach, must be taken into account using these kind of codes: the impossibility to represent three-dimensional flows, such as swirling flows in the flame tube or recirculating flows within dump diffuser or the end of the annulus, and the uncertainty related to the correlative calculation of pressure drop and heat transfer.

Nevertheless, 1D codes are essential for the thermal design of impingement or effusion cooled liners, where the high number of small holes entail a significant computational effort that would not allow to perform sensitivity analyses to geometrical parameters or operating conditions. Even if their CFD simulations could be possible exploiting lower order approaches aimed at reducing the number of mesh elements (see Chapter 4), 1D codes still remain the first choice for their preliminary design.

\subsubsection{Therm1D}

The previous section described the main features of $1 \mathrm{D}$ codes for the design of combustor cooling systems. Here below is instead presented the one-dimensional tool currently employed for the preliminary design of combustors: Therm1D. This code was developed starting from well proven approach for the thermal analysis of blades and vanes cooling systems. This tool is capable of estimating radiative and convective heat loads on 
both gas and coolant sides of the liners, with the aim of predicting the metal temperature.

The thermal calculation is based on the well known approach proposed by Lefebvre [7] and later improved by Kretschmer and Odgers [35] and De Champlain et al. 122. As described in Figure 5.1 the methodology relies in the balance among different mechanisms of heat transfer: radiative and convective heat loads on gas side ( $\mathrm{R} 1$ and $\mathrm{C} 1$ ), radiative heat load toward the casing (R2) and convective heat load between metal and coolant (C2). The energy balance in steady state condition implies that (neglecting heat conduction in axial and circumferential directions):

$$
R_{1}+C_{1}=R_{2}+C_{2}=K_{1-2}
$$

Once the hot and gas side boundary conditions are known, heat conduction within the metal $\left(\mathrm{K}_{1-2}\right)$ and its final temperature distribution is obtained using a 1D Finite Difference Model of the component with an in-house code integrated within the procedure.

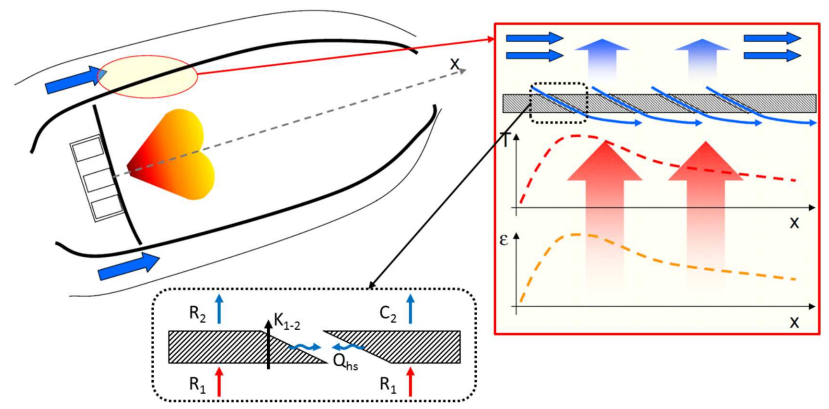

Figure 5.1: Conceptual illustration of 1D thermal analysis

Gas side heat loads are evaluated from a given description of the flame conditions in terms of FAR, temperature, velocity and pressure. Moreover, heat sink effect $\left(Q_{h s}\right)$ is accounted for applying, for each row, heat removal given by the calculated heat transfer coefficient and adiabatic wall temperature inside the perforation. 
After this introduction, some details concerning the coupling among the codes is given here below. The procedure is composed of three main parts: Icons1D (Internal COoling Network Solver), a fluid network solver for heat transfer evaluation on the interfaces between the metal and the coolant, Cowl, that estimates the radiative and convective heat loads on the gas side and radiative heat transfer between the liner and the casing, and the thermal solver, that computes the heat conduction through the metal and finally provides the wall temperature distribution.

The analysis of the coolant fluid network is performed using a onedimensional steady code, Icons1D, developed starting from a in-house code (SRBC) used in several works [123, 124, 125]. The representation of a cooling system consists of a fluid network made up connecting basic components, each one dedicated to model a particular region of the combustor. Specifications of the geometrical characteristics of a single component, such as holes diameter, pitch, length, roughness, inclination angle and so on, can be custom selected. Coolant is considered a perfect gas subjected to wall friction and heat transfer, and flow field is solved in subsonic regime, using correlations to determine HTC, friction factor, cooling effectiveness, etc. The user can specify boundary conditions for the fluid network in terms of inlet and outlet pressure or mass flow rate, depending on design specifications. Through the resolution of the fluid network, Icons1D provides coolant side boundary conditions for thermal calculation; in addition, it estimates also the heat sink effect of cooling holes.

Heat load on the gas side and radiative heat exchange between the liner and the casing are estimated by Cowl following the approach suggested by Lefebvre [7] and more recently improved by Andreini et al. [126]; afterwards, cold side and hot gas side boundary conditions are read by the thermal solver for the resolution of the Fourier law within the metal and the wall temperature is finally calculated. Since the mass flow rate and pressure drop is wall temperature dependent, an iterative overall procedure is required; in addition, an internal loop is included to speed up the convergence of the procedure. Convergence is achieved when 
differences in temperatures, pressures and mass flow remain unchanged or below an error set by the user. To have a better comprehension of the overall procedure and to clearly describe the connections among the different involved codes, a schematic flow chart is reported in Figure 5.2 . Further details on the procedure can be found in [126, 127.

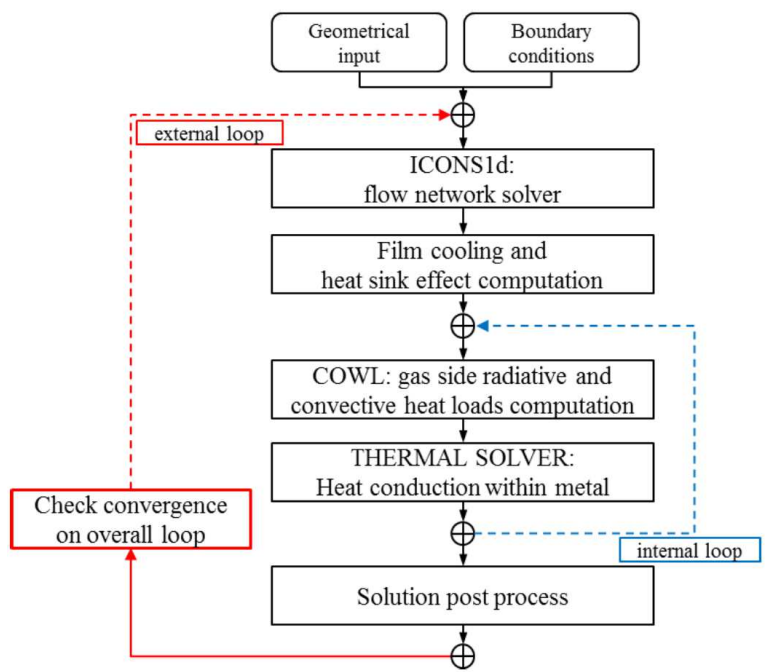

Figure 5.2: Therm1D: procedure flow chart

The estimation of thermal loads is carried out by means of a correlative approach, therefore the choice of the employed correlations plays a key role the prediction as accurate as possible of metal temperature. Here below are reported the equations implemented within the code.

\subsubsection{Gas side radiation}

The radiative heat transfer due to presence of flame and hot gases is treated making reference to two contributions: a non-luminous component emitted by gas, mainly given by $\mathrm{CO}_{2}$ and $\mathrm{H}_{2} \mathrm{O}$, and a luminous component related to soot production. 
The contribution of non-luminous radiation can be estimated starting from dimension and shape of the gas volume and its conditions in terms of pressure, temperature and chemical composition. The radiative heat load can be expressed as:

$$
R_{1}=0.5 \sigma\left(1+\epsilon_{w}\right) \epsilon_{g} T_{g}^{1.5}\left(T_{g}^{1.5}-T_{w 1}^{1.5}\right)
$$

Where $\epsilon_{w}$ is wall emissivity and depends on the material, temperature and oxidation level of the wall. Gas emissivity $\epsilon_{g}$ can be calculated using the expression proposed by Farag [128, valid for the lean combustion of kerosene for $P<40 \mathrm{bar}, 1200<T_{g}<2400 \mathrm{~K}, F A R<F A R_{s t}$ :

$$
\epsilon_{g}=\left(0.15-0.00005 T_{g}\right)\left(2 P F A R l_{b}\right)^{\left(0.20+0.00015 T_{g}\right)}
$$

Whereas the beam length $l_{b}$ is determined from the dimension of the combustion chamber using the expression:

$$
l_{b}=3.4 \frac{\text { Volume }}{\text { Surface }}
$$

It is worth remarking that actual gas temperature and composition are far from the homogeneous conditions, so the variables of Eq. 5.2 must be evaluated in terms of averaged values.

For what concerns luminous radiation due to soot formation, a general expression expresses total emissivity $\epsilon_{T}$ as the contribution of gas $\epsilon_{g}$ and soot $\epsilon_{s}$ :

$$
\epsilon_{T}=\epsilon_{g}+\epsilon_{s}-\epsilon_{g} \epsilon_{s}
$$

Since the evaluation of $\epsilon_{g}$ is based on the knowledge of soot volume fraction, which is difficult to estimate in a preliminary design phase, a simplified approach was proposed in [118, using the corrective factor Luminosity Factor $L F$, empirical function of the carbon level within the fuel.

$$
\epsilon_{T}=1-\left(1-\epsilon_{g}\right)^{L F}
$$




\subsubsection{Coolant side radiation}

The heat load transferred via radiation between liner and casing is significantly lower compared to the convective one. Nevertheless, it can be calculated assuming that both walls act like grey bodies. It is common practice to assume the casing temperature $T_{c}$ equal to coolant temperature at the entrance of the annuli $T_{3}$ :

$$
R_{2}=\sigma \frac{\epsilon_{w} \epsilon_{c}}{\epsilon_{c}+\epsilon_{w}\left(1-\epsilon_{c}\right)\left(A_{w} / A_{c}\right)}\left(T_{w 2}^{4}-T_{3}^{4}\right)
$$

For an annular chamber $A_{w} / A_{c}$ is equal to the ratio of the liner and casing diameters, hence $<1$ for the inner liner and $>1$ for the outer liner.

\subsubsection{Gas side convection}

Among the heat transfer processes occurring within the combustor, the convection between gas and liner certainly the most challenging from a modelling point of view. Within the flame tube gas is affected by rapid physical and chemical changes, as well as significant temperature and velocity gradient.

In absence of film cooling, it is reasonable to employ a classical correlation for Nusselt number in tubes, such as:

$$
C_{1}=0.020 \frac{k_{g}}{d_{h 1}^{0.2}} \frac{m_{g}}{A_{l} \mu_{g}}\left(T_{g}-T_{w 1}\right)
$$

Several factors must be considered for a correct estimation of wall temperature. The presence of a swirling flow leads to the generation of a complex flow field that can differ significantly from a fully developed flow within a tube, such as vortex breakdown phenomenon, corner recirculation zones and a swirled component in the proximity of the wall. To take into consideration this latter effect, gas velocity is increased by a $1 / \cos (\beta)$ factor, where $\beta$ represents the angle between velocity and the combustor axis. 


\subsubsection{Coolant side convection}

To estimate the convective heat transfer between liner and coolant it is possible to rearrange Eq. 5.8 using the hydraulic diameter of the annulus $D_{a n}$ and the fluid properties calculated at the air temperature $T_{3}$ :

$$
C_{1}=0.020 \frac{k_{c}}{d_{a n}^{0.2}} \frac{m_{a n}}{A_{a n} \mu_{c}}\left(T_{w 2}-T_{3}\right)
$$

\subsubsection{Heat sink}

The heat removal due to convection within the perforation can be of particular relevance for film cooling system, especially for effusion cooling, where it can contribute up to $30 \%$ to the overall cooling of the liner. Film cooling rows are usually modelled as equivalent slot and the total heat sink can be expressed as:

$$
Q_{h s}=A_{\text {slot }} h\left(T_{3}-T_{w}\right)
$$

Where the heat transfer coefficient $h$ can be calculated with an appropriate correlation for the Nusselt number. Due to the typical geometrical characteristics of the film cooled liners, the correlation should take into account the effect related to low $L / D$ ratio, such as the expression proposed by Gnielinski [129] for the range $2300 \leq R e \leq 10^{6}, 0.6 \leq P r \leq 2000$, $0<D / L<1$ :

$$
N u_{D}=\frac{(f / 2)\left(R e_{D}-1000\right) \operatorname{Pr}}{1+12.7(f / 2)^{1 / 2}\left(\operatorname{Pr}^{2 / 3}-1\right)}\left[1+\left(\frac{D}{L}\right)^{2 / 3}\right]
$$

\subsubsection{Film cooling performance}

The performance estimation of effusion cooling plates is a challenging task, deeply influenced by the superposition of the coolant due to the injection through multiple locations. For this reason, the most common approach is the evaluation of the film coverage provided by a single row, corrected subsequently with a criterion to manage the superposition 
phenomenon.

Within Icons1D are implemented the two correlations reported in open literature: those one developed by L'Ecuyer and Soechting 130] and Baldauf et al. 131. They were both developed for a single row of film cooling holes. The validity ranges of the main operating parameters are summarized in Tab. 5.1. Unfortunately both the correlation were developed for blade film cooling holes and, for example, the range of some parameters such as the blowing ratio are quite lower than typical values for combustor. It is worth noting that the ranges of the experimental database used by L'Ecuyer and Soechting are much wider than the ones used by Baldauf et al. for their correlation, which instead is based on more recent data and moreover it is sensitive to the mainstream turbulence level. Outside the validity range, the code extrapolates the behaviour of the correlation.

\begin{tabular}{cccc}
\hline & & L'Ecuyer and Soechting [130] & Baldauf et al. [131] \\
\hline Geometry & $\alpha$ & $30,35,45,55,90$ & $30,60,90$ \\
& $S_{y} / D$ & 2,3 & $2,3,5$ \\
& $L / D$ & $D[m m]=2-12.8$ & 6 \\
\hline Hot gas flow & $R e_{D}$ & $3500-52000$ & $6800-14000$ \\
& $T u$ & - & $1.5 \%, 4 \%$ \\
\hline Coolant flow & $B R$ & $0.1-3.75$ & $0.2-2.5$ \\
& $D R$ & $0.79-4.26$ & $1.2-1.8$
\end{tabular}

Table 5.1: Operating parameters for the implemented correlations

The superposition principle proposed by Sellers 132 is taken into account to predict the performance of multiple film cooling rows. According to this approach, the gas temperature involving the $i^{\text {th }}$ row is represented by the adiabatic wall temperature of the previous row $\left(T_{a w, i-1}\right)$, therefore for the adiabatic effectiveness of the $N^{\text {th }}$ row can be expressed as:

$$
\begin{array}{r}
\eta_{N}=\eta_{1}^{\prime}+\eta_{2}^{\prime} \cdot\left(1-\eta_{1}^{\prime}\right)+\eta_{3}^{\prime} \cdot\left(1-\eta_{1}^{\prime}\right) \cdot\left(1-\eta_{2}^{\prime}\right)+\ldots+ \\
+\eta_{N}^{\prime} \cdot\left(1-\eta_{1}^{\prime}\right) \cdot\left(1-\eta_{2}^{\prime}\right) \cdot \ldots \cdot\left(1-\eta_{N-1}^{\prime}\right)
\end{array}
$$

where: 


$$
\eta_{i}^{\prime}=\frac{T_{a w, i-1}-T_{a w, i}}{T_{a w, i-1}-T_{c}} \quad(i=1,2, \ldots, N-1)
$$

It is worth remembering that the superposition principle was developed for 2D slot cooling, therefore it is not able to properly take into account the increased mixing ascribable to the three-dimensional nature of coolant injection through discrete holes. Generally, this phenomenon involves a lower protection in comparison with the result predicted by the superposition method, which hence should be used with caution. As a consequence of this interaction, in fact, the influence of injection from a row does not extend indefinitely downstream but only for a certain length (i.e. a given number of rows). A this purpose Andreini et al. 133. propose to specify the effectiveness superposition length for each row of cooling holes.

\subsubsection{Final remarks}

The methodology currently employed for the preliminary design of combustor cooling systems has been showed in this chapter, highlighting the features that make this approach highly recommended for this design phase, when several configurations have to be investigated and a rough estimation of the metal temperature is acceptable. In the more advanced stages of the process, when the cooling scheme performance needs to be thoroughly verified, also at the aim of estimating the component durability, the degree of uncertainty could be no longer acceptable, bringing several approaches into question:

- Film cooling performance is assessed by means of flat plate correlations, which are inherently unable to consider the impact of swirling flow-liner cooling interaction.

- Classical correlations for the estimation of Nusselt number in smooth channel are employed to estimate the heat transfer coefficient, whereas the actual flow field characteristics are far away from fully developed conditions. Moreover, local suction/injection of coolant 
leads to significant heat transfer augmentation in the proximity of the holes (see Chapter 2 .

- An accurate estimation of metal temperature should not ignore radiative heat loads, which in particular conditions, e.g. at high pilot/main ratio or at very high OPR, can play a key role in the determination of the overall thermal load.

- The assumption of $1 \mathrm{D}$ conduction neglects the effect of the temperature gradients in axial and circumferential directions, resulting in 1D temperature profiles.

- At last, it is worth mentioning the highly tunable inputs that are greatly useful for the assessment of the sensitivity to driving parameters can be subject to the variability due to user-defined criteria for their evaluation.

In the light of these issues, a different approach has been proposed with the aim of increasing the accuracy in the metal temperature prediction, finalized at an accurate design of aero-engine combustor liners.

\subsection{Detailed design: Therm3D}

The main objective of this methodology is to represent an affordable alternative to a full CHT simulation of the entire combustor. In order to drastically reduce the computational effort, several modelling strategies have been exploited, e.g. the delimitation of the computational domain and the treatment of effusion cooling by means of a high-order model. Moreover, it is worth making clear that, at the moment, the methodology has been developed to be used in the framework of steady state simulations, bearing in mind, however, the possibility of potential future improvements towards transient simulations with more advanced turbulence models such as SAS. 


\subsubsection{Concept}

Here below are reported the strategies adopted to manage some critical aspects such as the estimation of hot and cold side boundary conditions and metal temperature calculation.

\subsubsection{Hot side BCs calculation}

With the aim of improving the accuracy of the metal temperature prediction, the main idea is the evolution from a correlative-based to a CFD-based solution, at least for what concerns the characterization of gas side boundary conditions, which, as pointed out in Chapter 3 , show all the limitations of the correlative approach. A more reliable estimation of radiative heat loads and heat transfer coefficient distribution, taking into account also the heat transfer augmentation due to the presence of effusion cooling, could be probably possible through a strong revision of the employed correlations. Nevertheless, the most critical issue is certainly represented by the evaluation of adiabatic effectiveness, which is heavily affected by the gas side flow conditions and characterized by non-localized phenomena, such as coolant superposition.

Therefore, it has been decided to assess gas side heat loads by means of a 3D CFD simulation of the combustor flame tube (Fig. 5.3). This way, swirling flow-liner cooling can be taken into account, whereas the application of radiative heat models considers the actual temperature field and the view factors between hot gas and surfaces. Nevertheless, the extensive use of effusion cooling imposes an appropriate treatment aimed at managing the number of mesh elements to an affordable level, therefore the mass injection is modelled with the source point approach presented in Chapter 4

The computational domain is delimited to the flame tube, thus contributing to limiting the computational effort and easing convergence, but requiring, on the other hand, a different approach for the calculation of the boundary conditions applied to the CFD domain, i.e. the flow split within the combustor. 


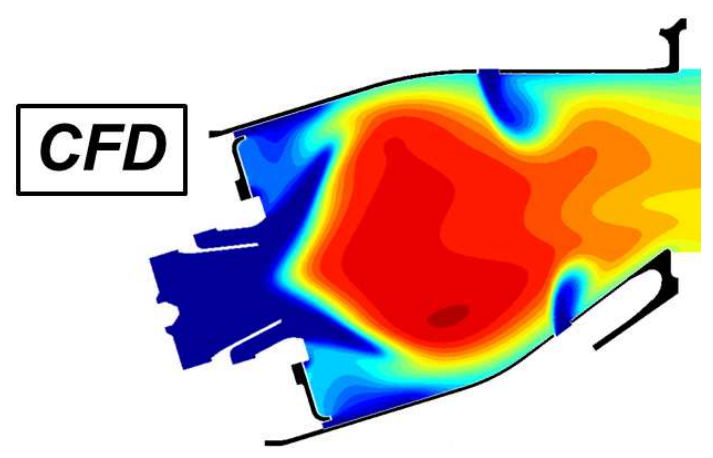

Figure 5.3: Sketch of CFD domain

\subsubsection{Cold side BCs calculation}

The remaining part of the combustor is modelled by means of a flow network solver (Fig. 5.4), which provides the solution of flow split within diffuser, cowl and annuli, as well as the calculation of the heat loads on the cold side, i.e. back side of the liners and within the perforation. This task is performed by means of appropriate models, aimed at creating the equivalent combustor flow network, and correlations for pressure drop (friction factor and discharge coefficient) and heat transfer (Nusselt number), which, despite the complexity of the flow field, can provide a reliable estimation though a $1 \mathrm{D}$ approximation.

\subsubsection{Thermal calculation}

In order to obtain the temperature distribution, appropriate boundary conditions must be applied to the component on which thermal conduction is calculated. This process has been performed contextually to the CFD solution, i.e. carrying out a Conjugate Heat Transfer (CHT) analysis.

The 3D CAD of the metal components are thus directly included within the CFD simulation and coupled to the fluid domain (Fig. 5.5). The resulting CHT approach involves the continuity of the heat flux across the fluid-solid interface, therefore the specification of appropriate 


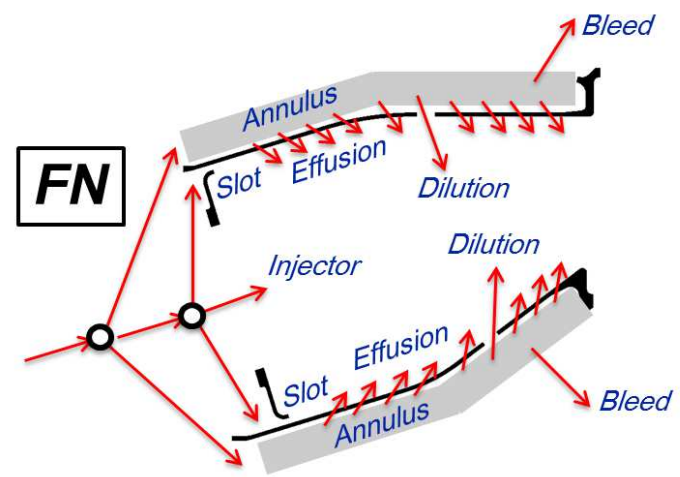

Figure 5.4: Sketch of flow network

boundary conditions are required only to the not-interfaced surfaces, e.g. on cold side and within the perforation, where heat transfer coefficient and adiabatic wall temperature distributions are imposed.

Moreover, the computational grid of the liners contains all the relevant features of the components, such as effusion cooling holes. This way, not only the macro-variations of temperature in the three directions are reproduced, but also the local gradients due to film injection and heat sink within the holes. The inclusion of the whole perforation certainly involves an increase in the total amount of mesh elements, without, however, significantly increasing the computational effort since only the energy equation is resolved within the solid domain.

\subsubsection{Development}

The coupling between CFD code (ANSYS CFX) and the flow network solver (Icons1D) is key for the accomplishment of the metal temperature prediction. The type of transferred data is illustrated in Fig. 5.6 for what concerns the flow split evaluation, the static pressure within the flame tube is passed to the flow network, which, given the total pressure within the annuli and the calculated discharge coefficient, returns a mass flow rate 


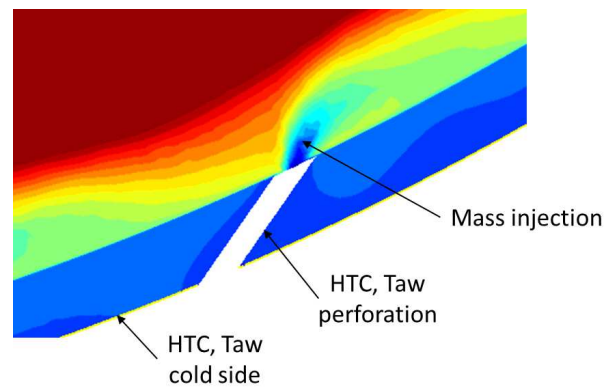

Figure 5.5: Sketch of boundary conditions applied to solid domain

of the film cooling / dilution row. Convective heat loads are calculated by the flow network, once passed the wall temperature by the CFD simulation, employing the correlation previously described for Therm1D. With the aim of accurately predict the heat transfer augmentation due to coolant suction, the $H T C_{0}$ on the back side of the liner is corrected by means of a specific correlation 134 that expresses an enhancement factor $E F=H T C / H T C_{0}$ as a function of pressure ratio $\beta=P_{0} / P$ and porosity of the plate $\sigma$.

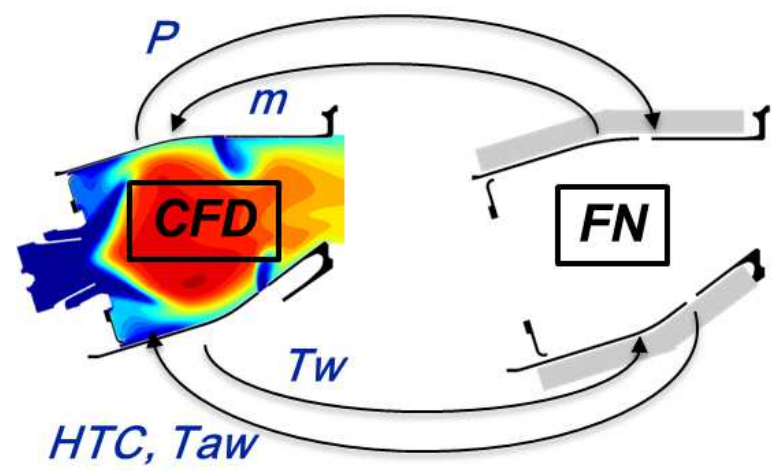

Figure 5.6: Sketch of codes coupling 
The entire coupling process is executed at run time during the CFD solution. The CFD simulation is performed, at the moment, in steady state and the boundary conditions exchange between the codes occurs every $\mathrm{N}$ iterations, where $\mathrm{N}$ is a value set by the user (see Fig. 5.7). ANSYS CFX allows the implementation of user defined Fortran subroutines (User Fortran) with the aim of expanding the code with additional features and physical models. A sketch of the implementation process is depicted in Fig. 5.8 .

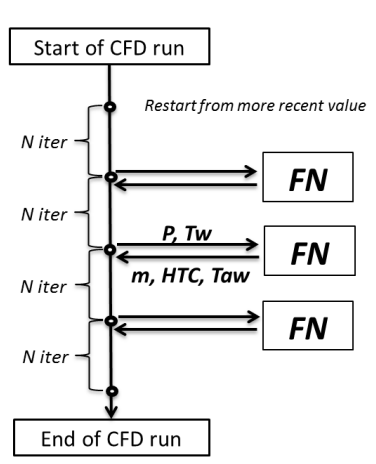

(a) Sketch of solution process

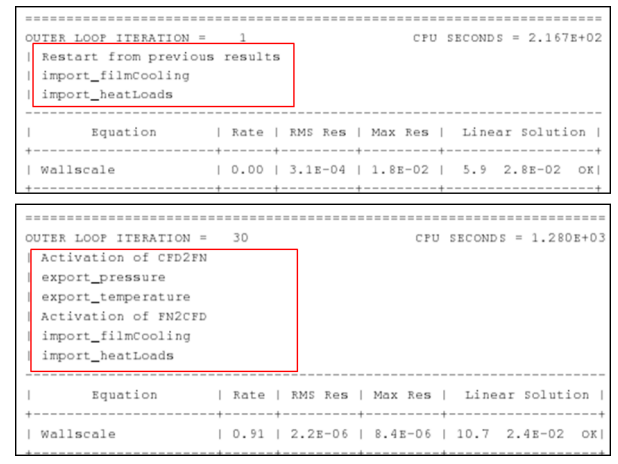

(b) Example of user subroutine run-time activation

Figure 5.7: Coupling between CFD and Flow Network (FN)

- Definition of a user CEL routine and user CEL function object

- Implementation of the user function as a CEL expression

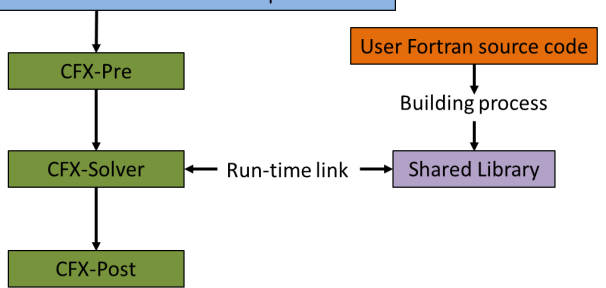

Figure 5.8: Implementation of User Fortran code in CFX 


\subsubsection{Mass flow rate calculation}

In more detail, the procedure for the calculation of mass flow rate is reported below, with particular emphasis on the solutions employed to run the CFD solution in parallel.

1. Calculation of the geometrical position of the point downstream of a hole

2. Individuation, for each partition, of the closest mesh node to the above mentioned point

3. Calculation, for each partition, of the static pressure value in that mesh node and exportation to output file

4. Choice of the value calculated on the mesh node at absolute minimum distance

5. Calculation of the arithmetic mean among the values of each row

6. Application of updated boundary conditions to flow network

7. Solution of the flow network and calculation of the mass flow rate of the row

8. Application of mass flow rate to each source point

\subsubsection{Cold side BCs calculation}

Also the calculation of thermal loads is performed through User Fortran subroutines according to the following procedure:

1. Calculation of average wall temperature of each row

2. Calculation of a $1 \mathrm{D}$ wall temperature profile for the cold side of the liner

3. Application of updated boundary conditions to flow network

4. Solution of the flow network and calculation of the heat loads for the row

5. Application of heat loads to the CFD simulation 


\subsection{Final remarks}

The proposed methodology is presented in this chapter, with particular focus on the design strategies adopted, keeping in mind the objectives to be achieved. Each choice has been motived, underlining resultant advantages and drawbacks. An application of Therm3D is reported in the following chapter. 



\section{Chapter 6}

\section{Application of Therm3D to NEWAC Combustor}

This chapter reports the main findings resulting from the application of Therm3D to a test case. In particular, the test case chosen for the validation is the same one already presented: the NEWAC annular combustor. For this reason, it is possible to refer to Chapter 4 for a more detailed description.

\subsection{Numerical settings}

\subsubsection{CFD simulation}

The CFD setup is roughly unchanged in respect to the simulations carried out with SAFE methodology on the same test case (see Chapter 4). 3D CFD RANS conjugate calculations have been considered in this study, referring to the Navier-Stokes equations solver ANSYS ${ }^{\circledR}$ CFX v15. Compressibility effects have been taken into account and a High Resolution advection scheme has been used, which automatically blends between first and second order scheme throughout the domain based on the local solution field [113. The fluid has been modelled as a reacting mixture, whereas thermal conductivity and emissivity were specified for 
the metal properties. Energy equation has been solved in terms of total energy and viscous heating effects have been accounted for.

This time, the k- $\omega$ SST turbulence model has been used with the aim of better representing the heat transfer in the proximity of the wall. In order to overcome the issue represented by the above mentioned ignition within the injector, the fuel flow split has been changed from 0.95 to 1.0. Even if this approximation could leand to local alterations in the temperature field, it is considered to be acceptable for the purposes of this work, focuses on gas side heat transfer (dominated by convection) rather than $\mathrm{NO}_{x}$ emissions.

The considered fuel is JetA. A conventional eulerian-lagrangian discrete particle model was employed to track JetA liquid fuel. Fuel is injected directly at the end of the lip and secondary particle break-up has been modelled using the Cascade Atomization and Breakup Model (CAB), which represents a development of TAB model. Combustion is treated with the combined Finite Rate Chemistry/Eddy Dissipation model, which computes the reaction rates for each model separately and then uses the minimum of the two, and modelled by means of a two-step kinetic scheme. Both turbulent Prandtl and Schmidt numbers have been set to 0.9 , so as to obtain a Lewis number equal to 1 , with the aim of fully respecting the mass-energy transfer analogy. The impact of the radiative heat transfer has been evaluated through a comparison of different models implemented in the code, namely the Discrete Transfer and the Monte Carlo models.For what concerns the spectral model, a weighted sum of gray gases (WSGG) has been exploited. In the context of this work, four different radiating gases have been considered.

A sketch of the computational domain is reported in Fig. 6.1, which is delimited to the combustor flame tube. Flow split and temperature of the fluid at each inlet is provided by the flow network solution, whereas profiles of velocity components and turbulence quantities, deriving from the CFD simulation of the entire combustor, are assigned at the patches of swirler and dilutions rows. A static pressure value has been imposed at the outlet. In order to reduce the computational effort, the domain 
has been limited to a $20^{\circ}$ sector of the single annular combustor and a periodic condition has been imposed. Fluid and solid domain have been interfaced through General Grid Interface (GGI) across the liners in order to perform a Conjugate heat transfer (CHT) simulation, whereas adiabatic conditions have been applied to all other surfaces. The fuel injection has been characterized by means of temperature, direction and velocity magnitude of the particles, while a Rosin-Rammler distribution was considered for the droplet size.

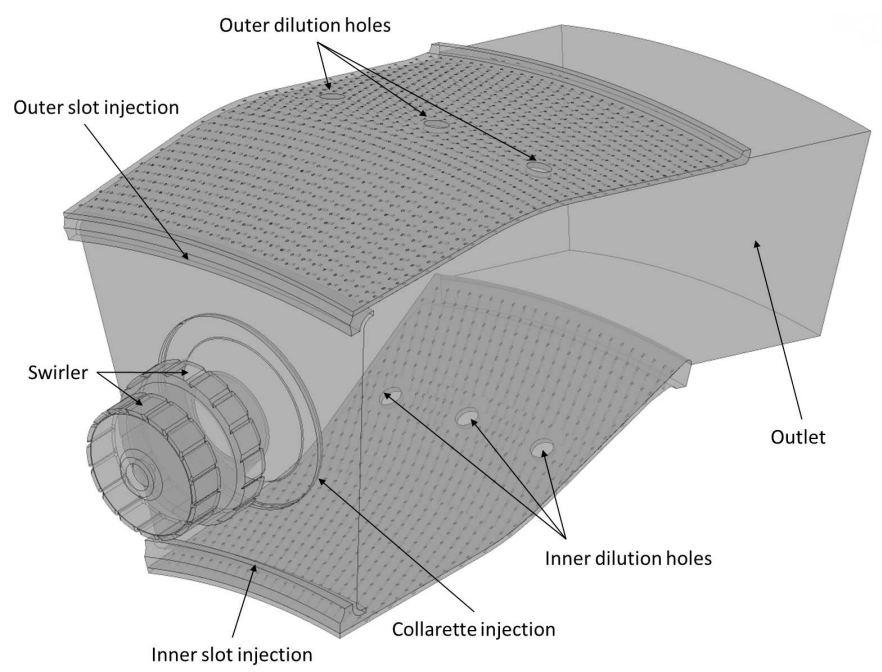

Figure 6.1: Sketch of the computational domain

In order to track the spatial distribution of the coolant, an additional transport equation was added for a passive scalar Coolant representing coolant mass flow, whose values are 1 for the flow coming from the source points and 0 at the inlets of the domain.

The software ANSYS ${ }^{\circledR}$ ICEM-CFD has been used to generate the computational grids, realized with an increasing refinement level in order to perform an assessment of the mesh sensitivity (see Tab. 4.6). These 
grids are characterized by tetrahedrons with 10 layers of prisms close to the wall, whereas the mesh of the solid domain, composed of tetrahedrons, counts about 20 million elements, since the perforations of both liners have been explicitly reproduced. A mesh sensitivity analysis has been carried out in order to establish the adequate level of mesh refinement to capture the flow physics and is reported in the following. Some details and the pictures of the computational grids are reported in Tab. 6.1. The grids, see Figs. 6.2 have been generated keeping the same mesh sizing level on the walls and increasing refinement within the fluid volume, with the aim of better discretizing the high gradients characteristic of swirling flows.

\begin{tabular}{cccc}
\hline \hline & Min size $(\mathrm{mm})$ & Elements $\left(10^{6}\right)$ & Nodes $\left(10^{6}\right)$ \\
\hline Mesh 1 & 1.5 & 13.32 & 4.03 \\
Mesh 2 & 1.0 & 15.27 & 4.38 \\
\hline \hline
\end{tabular}

Table 6.1: Main features of the computational grid

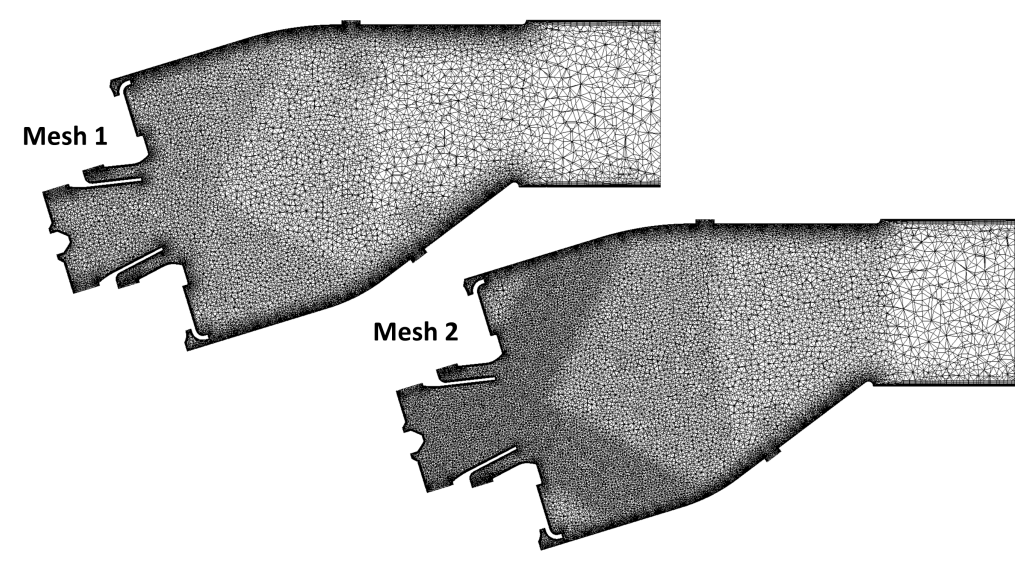

Figure 6.2: computational grids for mesh sensitivity analysis

The solution convergence has been assessed by monitoring the residuals, 
the imbalance across the domains and appropriate monitor points of pressure and temperature in representative locations. Simulations have been stopped when these trends reached a steady-state.

\subsubsection{Flow network}

The equivalent flow network of the combustor is depicted in Fig. 6.3 As it is possible to notice, the flow network consists mostly of flow splitting elements and specific models for smooth channels, impingement cooling and annuli with effusion cooling.

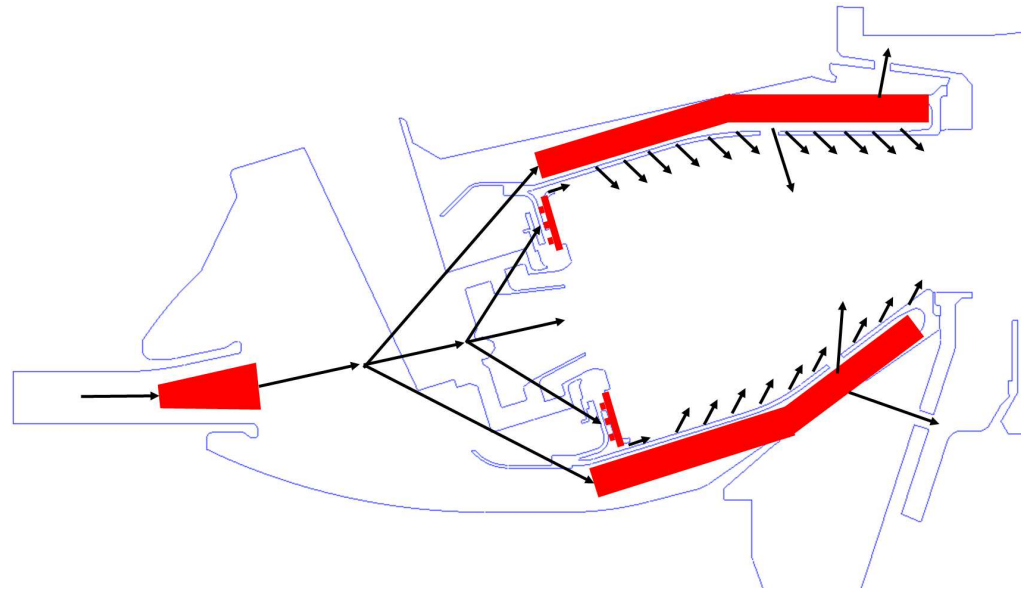

Figure 6.3: Sketch of equivalent combustor flow network

Boundary conditions are imposed in terms of mass flow rate and total temperature at inlet of the network, i.e. the compressor discharge, static pressure value at swirler discharge and the design target flow split and bleeding ports. Due to the difficulties in reproducing the three-dimensional nature of the flow field within the dump diffuser, some loss coefficients are tuned in order to obtain the actual flow split between cowl and annuli.

The flow split within the annulus is then determined by the predicted discharge coefficients for effusion cooling and dilution holes. For what 
concerns effusion, the $C_{D}$ of each row is calculated using the already mentioned CFD-based correlation developed by Andreini et al. [115]:

$$
C_{D, i}=a_{0}+a_{1} \cdot \dot{m}_{r} \cdot\left(1+a_{2} \cdot V_{h r, i}\right)+a_{3} \cdot \frac{R e_{a n, i}}{R e_{h, i}}
$$

where $V_{h r}=\frac{P_{0, a n}-P_{a n}}{P_{0, a n}-P_{g}}$ is the Velocity head ratio, $\dot{m}_{r}=\frac{\dot{m}_{a n, i n} \sqrt{R T_{0}}}{P_{0} A}$ the flow coefficient and $R e_{a n} / R e_{h}$ the ratio between Reynolds numbers of annulus and hole. The discharge coefficient of dilution holes is instead estimated thorough the correlation for $90^{\circ}$ holes developed by Da Soghe and Andreini 135

Convective heat load is instead estimated using a classical correlation for the Nusselt number, such as that one proposed by Dittus-Boelter correlation [136 and corrected with a specific correlation for the augmentation ascribable to the presence of effusion cooling [134].

\subsection{Results}

The outcomes derived from the application of the methodology to the test case are reported here below. The results are presented starting from the mesh sensitivity analysis and then moving to the assessment about the impact of radiation modelling.

Since the objective of this work is the application of the proposed thermal methodology, the analysis has been based on the predicted metal temperature distribution along the liners, expressed in terms of $T^{*}=$ $T / T_{\text {ref }}$ vs $x^{*}=x / x_{\text {ref }}$. The available experimental data consists of temperature measurements obtained by means of thermocouples in the locations indicated in Fig. 6.4. For what concerns the tangential position, the data showed are placed on the center plane that crosses the injector. It is worth noticing that for certain axial locations multiple measurements are available, since several sectors have been instrumented. 


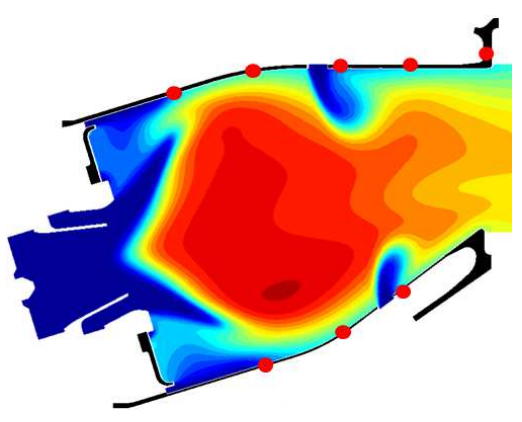

Figure 6.4: Position of thermocouples

\subsubsection{Mesh sensitivity}

The extracted wall temperature profiles are reported in Fig. 6.5, for outer 6.5a and inner 6.5b liners separately. Making reference to the outer liner, the profiles can be subdivided into 3 main regions:

- $0.62 \leq x^{*} \leq 0.70$ : located in the region of the corner vortex, this portion of the liner benefits of the high protection provided by the slot and the low gas temperature, due to significant mixing, resulting in poor convective thermal loads.

- $0.70 \leq x^{*} \leq 0.85$ : the strong temperature gradients in axial direction is ascribable to the interaction between swirling flow and liner, which involves hot gas at high velocity in the proximity of the wall.

- $0.85 \leq x^{*} \leq 0.98$ : consist in last part of the liner, downstream of the dilution holes, where good coolant protection is provided by superposition, though characterized by enhanced mixing due to air addition. Wall temperature further increases at the end of the combustor in correspondence of the minimum cross-sectional area, as a result of the acceleration of hot gas.

The resulting agreement against experimental data is generally good for what concerns the outer liner, whereas huge discrepancies can be pointed out for the inner liner. The CFD profiles shows coherent behaviours, 
in terms of both magnitude and trend, whereas the geometrical and flow characteristics do not seem to explain such a gap, in the order of $250 \mathrm{~K}$. Unfortunately the limited number of thermocouples in the circumferential direction does not allow to understand the reliability of the single measurement, nevertheless, the tests carried out on the test articles did not showed particular signs of damages in the specific region.

Concerning the sensitivity to mesh refinement, minor discrepancies are highlighted between the two grids, except for a delimited region of the inner liner, where the temperature values predicted with the finer mesh, however, move further away from the trend depicted by experimental data.

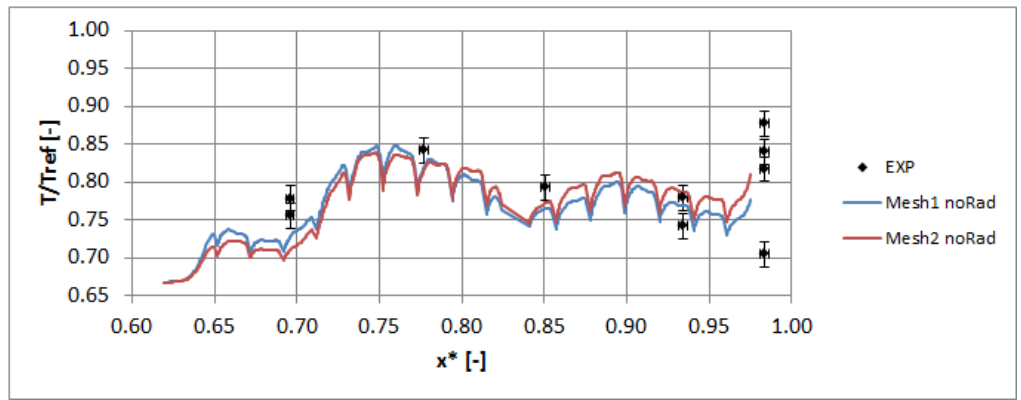

(a) Outer liner

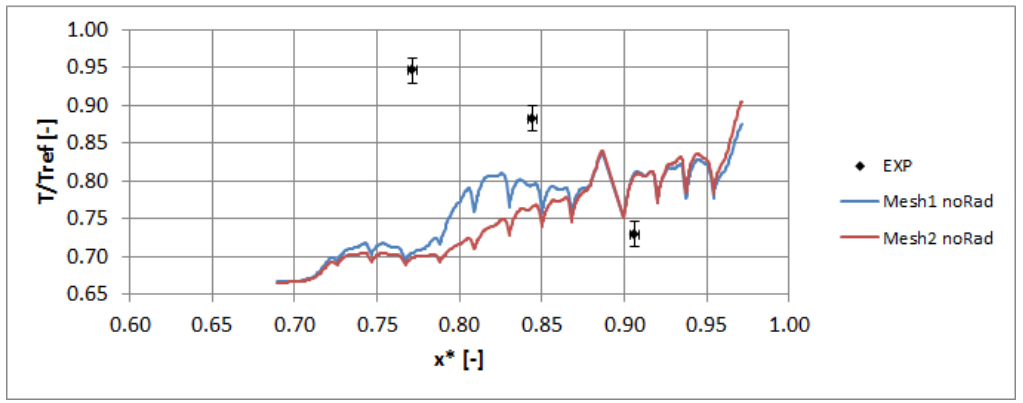

(b) Inner liner

Figure 6.5: Mesh sensitivity analysis: metal temperature distribution on cold side 


\subsubsection{Radiation modelling sensitivity}

With the aim of assessing the impact of radiation on the predicted metal temperature distribution, two models have been tested and compared against the previous results obtained without taking into account the radiation heat transfer, namely the Discrete Transfer (DT) and the Monte Carlo (MC) models.

As it is possible to notice making reference to Fig. 6.6 radiation has roughly a constant contribution along the liners. The effect can be estimated at around $50 \mathrm{~K}$, independently from the radiative model used, therefore it is possible to conclude that radiative heat transfer plays a minor role in determining the metal temperature distribution, at least for the test case and the operating conditions under consideration. A more thorough assessment, which however does not represent the main objective of this work, would require further tests carried out at different test points.

\subsection{Potential improvements}

The obtained results give an insight on the main qualities of the proposed approach, as well as on the perspectives for further improvements. Here below is reported a list of aspects that would deserve additional investigations aimed at an enhancement of the methodology.

\subsubsection{Effusion cooling modelling}

The effusion cooling model based on the local injection by means of point sources has clearly shown some issues related to the mis-prediction of the injection angle, leading to discrepancies in the estimation of convective heat loads. The solution proposed is based on the exploitation of an advanced model with sources distributed over multiple mesh elements. For the sake of clarity, it is possible to refer to Fig. 6.7. which depicts the mesh elements on which the distributed mass source would be applied.

A first noticeable effect of the application of this new film cooling model is highlighted in Fig. 6.8, where the impact on the velocity distribution 


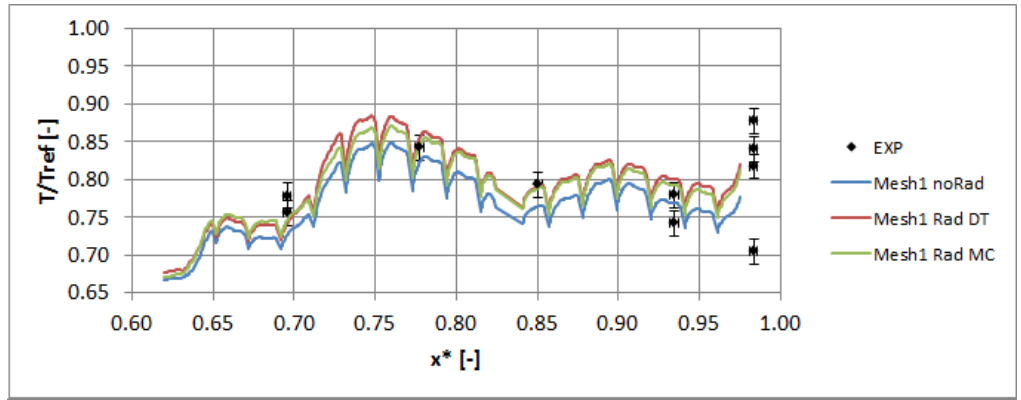

(a) Outer liner

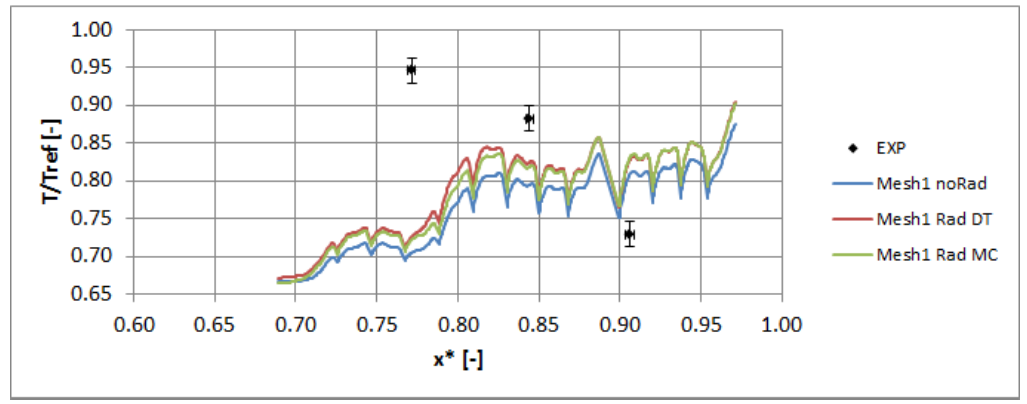

(b) Inner liner

Figure 6.6: Radiation modelling sensitivity analysis: metal temperature distribution on cold side

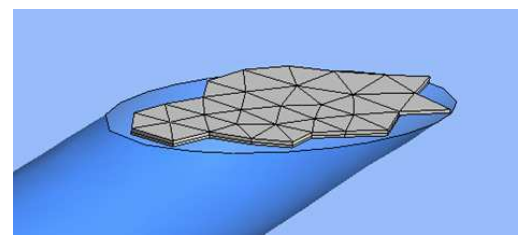

Figure 6.7: Distributed mass source model: mesh elements for source application

downstream of an effusion cooling plate is shown. It appears evident that the new approach returns a more accurate prediction of jet trajectory and 
overall flow field.

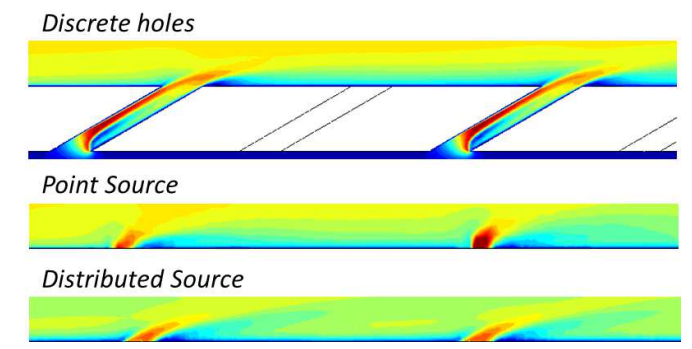

Figure 6.8: Comparison between effusion cooling models: span-wise averaged $\eta_{\text {aw }}$ : flow field

In order to provide a more quantitative comparison, the span-wise averaged distributions returned by point (PS) and distributed (DS) source models are reported in Fig. 6.9 The comparison against experimental data and CFD results obtained by a simulation with discrete holes highlight how a better agreement can be achieved with an advanced film cooling model. It is worth pointing out that through a mesh refinement (increasing from 2 up to 4 elements per hole diameter) the behaviour of the discrete holes can be roughly reproduced, therefore more accurate results can be achieved provided that an adequate mesh refinement is used. Moreover, it is worth pointing out that a gap is still present in respect to experimental data (especially at low BR), but this aspect is to be ascribed to turbulence modelling, rather than the effusion model.

\subsubsection{Turbulence modelling}

Even if the most accurate effusion cooling model would be used, the estimation of the thermal loads would be affected by the employed turbulence model. As remarked several times during this dissertation, RANS approach is inherently unable to reproduce some effects of strongly unsteady phenomena. Several modifications have been proposed in open literature to overcome the limitations of classical models, e.g. the imple- 


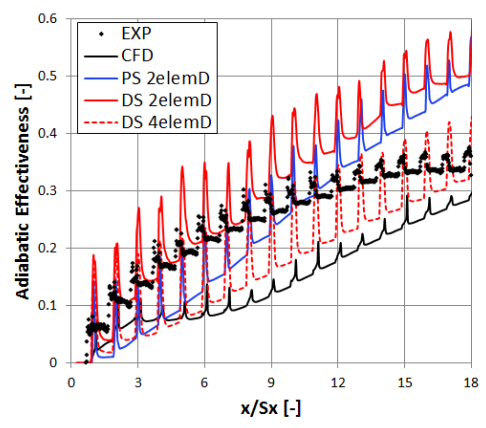

(a) $B R=1$

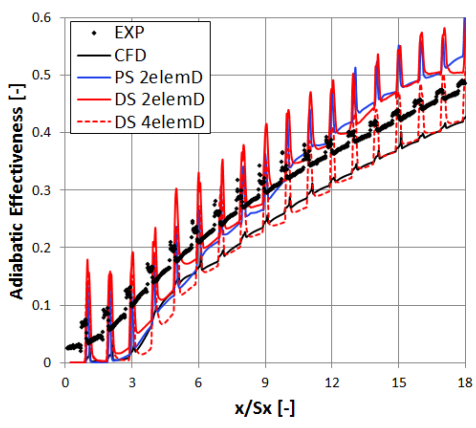

(b) $B R=3$

Figure 6.9: Comparison between effusion cooling models: span-wise averaged $\eta_{\text {aw }}$ : adiabatic effectiveness

mentation of an anisotropic formulation for eddy viscosity. Nevertheless, these models are hardly exploitable for complicated geometries, such as a combustor or a film cooled nozzle/blade.

The increasing computational resources are allowing the perform simulations with higher order turbulence models. The promising results highlighted by hybrid RANS-LES models (see Chapter 3 ) suggest a transition towards this kind of approaches. In this perspective, Therm3D would require minor modifications to be applied in conjunction with transient CFD simulations, e.g. the revision of the coupling strategy between fluid and solid domain.

\subsubsection{Radiative heat transfer}

In order to increase the accuracy in the estimation of radiative heat loads, several actions could be undertaken: since the radiative heat load depends exponentially from gas temperature, a more realistic representation of the temperature field would benefit from the employment of more advanced turbulence models, as already said, and combustion models with more detailed reaction mechanisms. Moreover, the contribution of soot is not taken into account at the moment, which presence can be significant 
for particular engines, such as RQL, or operating conditions. In order to overcome this lack, a widespread model proposed by Balthasar et al. 137. could be implemented, describing the formation and oxidation of soot particles.

\subsubsection{Flow network}

Further improvements can be provided also for the flow network modelling of the cold side of the plate, mainly aimed at enhancing the accuracy in the prediction of flow split and thermal loads through a revision of implemented correlations. Moreover, Icons1D provides for advanced capabilities, such as a multi-section mode, which allows to further subdividing the investigated geometry in multiple sections, so as to better characterize the variation of gas side conditions in the circumferential direction. Modifications could be implemented in the coupling strategy so as to calculate the mass flow rate of each cooling hole starting from static pressure profiles that vary in circumferential direction.

\subsection{Final remarks}

In this chapter an application of the proposed thermal methodology to a representative test case is presented. The achieved results have been showed through a comparison against experimental data, focusing on the influence of relevant parameters such as mesh refinement and radiation modelling. The analysis pointed out a limited impact on the metal temperature distribution, that, however, is characterized by huge discrepancies along the central part of the inner liner. Some doubts arise concerning the reliability of the experimental data, therefore additional investigations would be necessary on more detailed test cases.

Several aspects that would deserve further work have been identified and they are already under investigation. Anyhow, the methodology should be validated on additional test cases, preferably with different typologies of experimental data, so as to obtain a greater confidence on the reliability of the results. 



\section{Conclusions}

The research activity presented in this dissertation is aimed at deepening the knowledge concerning the heat transfer in ultra low $\mathrm{NO}_{x}$ combustors. The development of low-emission aero-engine combustors is indeed characterized by significant efforts made by all manufacturers in view of the demanding emissions limit set by ICAO-CAEP. In this scenario, lean burn concept is considered the most effective way to achieve the reduction target in pollutant emissions for future aero-engines. In particular, up to $70 \%$ of the overall air mass flow is dedicated to the combustion process, whereas the coolant is reduced of roughly $50 \%$. This, in conjunction with the trend based on the increase in turbine inlet temperature and overall pressure ratio, involves technological issues related to the thermal management of combustor walls.

For these reasons, lean combustors require very effective cooling schemes to cope life targets and, amongst the numerous solutions proposed in literature, effusion cooling has been identified by several manufacturer as one of the most promising solutions, capable of providing both excellent thermal performance and acoustic damping. The design of effective and reliable cooling schemes requires a detailed knowledge concerning both geometrical and operating parameters influencing the thermal performance and, most of all, the fluid dynamics conditions within the flame tube.

At first, a CFD campaign was performed on three different test cases: effusion cooled flat plates, an effusion cooled tubular combustor and a slot cooled annular combustor. The first analysis allowed to validate the CFD tool, assessing the performance of an improved version of the standard 
$k-\omega$ SST turbulence model. This modification was proposed to take into account the anisotropy of turbulence in the near-wall region, so as to mitigate the typical lateral spreading under-prediction deriving from the simulating film cooling through classical eddy viscosity models. Obtained results showed significant improvements for $30^{\circ}$ holes, but, unfortunately, this kind of approach is hardly exploitable for more complicated geometries such as those characteristic of industrial applications.

Once validated the methodology, the tool has been employed to investigate a more representative configuration, i.e. an effusion cooled tubular combustor, equipped with an ultra low $\mathrm{NO}_{x}$ swirler, namely the PERM injector designed by GE Avio. It was highlighted that a realistic flow field leads to major modifications in the near-wall region, especially for what concerns the outer recirculation zone, which, depending on the inclination angle of the perforation, can invert the direction of the corner vortex. This, in addition to the impingement of the swirling flow with the liner, returns a completely different coolant protection, if compared to previous results obtained from flat plates. Therefore, these findings point out the limits of simplified approaches for the estimation of adiabatic effectiveness distributions, which are usually carried out by means of flat plate correlations.

Since a reliable estimation of wall temperature requires an accurate assessment of both adiabatic effectiveness and heat transfer coefficient, the attention has been moved, at last, to the capabilities of CFD in predicting the heat transfer augmentation due to the interaction between swirling flow and liner. This activity allowed to better understanding the issues related to the simulation of swirling flow at non-reactive conditions in not confined configurations. Moreover, the very good agreement achieved with hybrid RANS-LES models shows promising perspectives about LES-like results with a reduced computational effort.

The main objective of the work is however the development of a methodology for the detailed design of effusion cooled combustors. With the aim of providing an affordable solution for the CFD simulation of this kind of devices, a source-based model was developed. The SAFE 
(Source based effusion model) methodology allows the simulation of the entire combustor, where the perforation is replaced injecting coolant through point sources. The mass flow rate is calculated exploiting a formulation dependant on local flow conditions and heat sink effect within the perforation is accounted too. The approach showed good capabilities in estimating flow split and pressure drop across the combustor, whereas some inherent limitations related to the point injection arose.

A further step towards the achievement of a trade-off between accuracy and reduced computational cost is achieved through the development of Therm3D, a thermal methodology based on the CFD simulation of the flame tube, in conjunction with the solution of the combustor equivalent flow network aimed at calculating the flow split and the thermal loads on the cold side of the liners. The application to an annular lean burn, effusion cooled combustor highlighted an overall good agreement on the outer liner, whereas major discrepancies are achieved against experimental data of the inner liner, whose reliability is questioned.

In conclusion, several aspects deserving to be further investigated have been indicated. It is worth mentioning, for example, a revision of the film cooling model, as well as the correlations implemented within the flow network solver. Moreover, compatibly to the computational resources, more accurate turbulence and combustion models should be employed to provide a more realistic representation of flow and temperature fields within the combustor. At this purpose, additional investigations will be carried out in the future on more detailed test cases. 



\section{Bibliography}

[1] Ballal, D. R. and Zelina, J. Progress in aeroengine technology (1939-2003). AIAA Journal of Aircraft, 41(1):43-50, February 2004.

[2] Airplanes, Boeing Commercial. Current market outlook 2007, 2007.

[3] Advisory Council for Aeronautical Research in Europe. Strategic research agenda. Technical report, ACARE, 2000.

[4] Schulz, A. Combustor liner cooling technology in scope of reduced pollutant formation and rising thermal efficiencies. Heat transfer in Gas Turbine Systems, 934:135-146, 2001.

[5] Ford, C. L., Carrotte, J. F., and Walker, A. D. The impact of compressor exit conditions on fuel injector flows. ASME J Therm Eng Gas Turb Pwr, 134(111504), November 2012.

[6] Wurm, B., Schulz, A., Bauer, H.J., and Gerendas, M. Impact of swirl flow on the cooling performance of an effusion cooled combustor liner. ASME J Gas Turb Pwr, 134(121503), 2012.

[7] Lefebvre, A. H. Gas Turbine Combustion. Taylor \& Francis, 1998.

[8] Advisory Council for Aeronautics Research in Europe. Aeronautics and air transport: Beyond vision 2020 (towards 2050): Background document. Technical report, Europ. Comm. Directorate-General for Research Aeronautics and Air Transport, 2010. 
[9] Carrotte, J. Aerodynamics, unsteadiness and mixing. In Combustion in aero-engines, Lecture Series 2012-04. Von Karman Institute for Fluid Dynamics, 2012.

[10] LEMCOTEC European Project. Description of work and internal reports. Confidential documents, 2011.

[11] Lieuwen, T. C. and Yang, V. Gas Turbine Emissions. Cambridge University Press, 2013.

[12] McKinney, R.G., Sepulveda, D., Sowa, W., and Cheung, A.K. The pratt \& whitney TALON X low emissions combustor: revolutionary results with evolutionary technology. AIAA Journal, (AIAA 2007386), January 2007). The 45th AIAA Aerospace Sciences Meeting.

[13] Rohde, J. Overview of the NASA AST and UEET emissions reduction projects, March 2002.

[14] General Electric. Taps II combustor final report - continuous lower energy, emissions and noise (CLEEN) program. Technical report, Federal Aviation Administration, June 2013.

[15] Bock, S., Horn, W., and Sieber, J. "Active Core" - a key technology for more environmentally friendly aero engines being investigated under the NEWAC program. In 26th International Congress of the Aeronautical Sciences, number 636, 2008.

[16] Correa, S. M. Power generation and aeropropulsion gas turbines: From combustion science to combustion technology. Symposium (International) on Combustion, 27:1793-1807, 1998.

[17] Lilley, D. G. Swirl flows in combustion: A review. AIAA Journal, 15(8):1063-1078, 1977.

[18] Lucca-Negro, O. and O'Doherty, T. Vortex breakdown: a review. Progress in Energy and Combustion Science, 27(4):431-481, 2001. 
[19] Syred, N., O'Doherty, T., and D., Froud. The interaction of the precessing vortex core and reverse flow zone in the exhaust of a swirl burner. Proceedings of the Institution of Mechanical Engineers, Part A: Journal of Power and Energy, (208):17-36, 1994.

[20] Vu, B. T. and Gouldin, F. C. Flow measurement in a model swirl combustor. AIAA Journal, 20:642-651, 1982.

[21] Rhode, D.L., Lilley, D.G., and McLaughlin, D.K. Mean flow fields in axisymmetric combustor geometries with swirl. AIAA Journal, 21:593-600, 1983.

[22] Ferrell, G. B., Abujelala, M. T., Busnaina, A. A., and Lilley, D. G. Lateral jet injection into typical combustor flow fields. AIAA 22nd Aerospace Sciences Meeting, 1984.

[23] Spencer, A., Hollis, D., and Carrotte, J. PIV measurements of combustor turbulence fields. In Proc. of ASME Turbo Expo, number GT2007-28050, 2007.

[24] Spencer, A., Hollis, D., and Gashi, S. Investigation of the unsteady aero-dynamics of an annular combustor using PIV and LES. In Proc. of ASME Turbo Expo, number GT2008-50277, 2008.

[25] Gnirb, M. and Tropea, C. Simultaneous PIV and concentration measurements in a gas-turbine combustor model. Exp Fluids, 45(4): 643-656, 2008.

[26] Marinov, S., Kern, M., Merkle, K., Zarzalis, N., Peschiulli, A., Turrini, F., and Sara, O. N. On swirl stabilized flame characteristics near the weak extinction limit. Proceedings of ASME Turbo Expo, (GT2010-22335), 2010.

[27] Kern, M., Marinov, S., Habisreuther, P., Zarzalis, N., Peschiulli, A., and Turrini, F. Characteristics of an ultra-lean swirl combustor flow by LES and comparison to measurements. Proceedings of ASME Turbo Expo, (GT2011-45300), 2011. 
[28] Wurm, B., Schulz, S., Bauer, H.-J., and Gerendas, M. Impact of swirl flow on the cooling performance of an effusion cooled combustor liner. ASME J Eng Gas Turb Power, 134, December 2012.

[29] Mellor, A. M. Design of Modern Gas Turbine Combustors. Academix Press, 1990.

[30] Gerendás, M., Höschler, K., and Schilling, Th. Development and modeling of angles effusion cooling for the BR715 low emission staged combustor core demonstrator. In RTO AVT Symposium on "Advanced Flow Management: Part B - Heat Transfer and Cooling in Propulsion and Power Systems". NATO Research and Technology Organisation, May 2001.

[31] Han, J. c., Dutta, S., and Ekkad, S. V. Gas Turbine Heat Transfer and Cooling Technology. Taylor \& Francis, first edition edition, 2000 .

[32] Andrews, G. E., Bazdidi-Tehrani, F., Hussain, C. I., and Pearson, J. P. Small diameter film cooling hole heat transfer: The influence of hole length. In Proc. of ASME Turbo Expo, number 91-GT-344, 1991.

[33] Andrews, G. E., Khalifa, I. M., Asere, A. A., and Bazdidi-Tehrani, F. Full coverage effusion film cooling with inclined holes. In Proc. of ASME Turbo Expo, number 95-GT-274, 1995.

[34] Gustafsson, K. M. B. and Johansson, T.G. An experimental study of surface temperature distribution on effusion-cooled plates. ASME J Gas Turb Pwr, 123:308-316, 2001.

[35] Kretschmer, D. and Odgers, J. A simple method for the prediction of wall temperatures in a gas turbine combustor. Proc. of ASME Turbo Expo, (78-GT-90), 1978.

[36] Goldstein, R. J. Film cooling. Advances in Heat Transfer, 7:321-379, 1971. 
[37] Kelly, G. B and Bogard, D. G. An investigation of the heat transfer for full coverage film cooling. Proc. of ASME Turbo Expo, (GT200338716), 2003.

[38] Sen, B., Schmidt, D. L., and Bogard, D. G. Cooling with compound angle holes: Heat transfer. ASME J Turbomach, 118:800-806, 1996.

[39] Andrews, G. E., Asere, A. A., Gupta, M. L., and Mkpadi, M. C. Full coverage discrete hole film cooling: influence of hole size. In Proceedings of ASME Turbo Expo, number 85-GT-47, 1985.

[40] Martiny, M., Schulz, A., and Wittig, S. Full-coverage film cooling investigations: adiabatic wall temperatures and flow visualizations. In Proceedings of ASME Turbo Expo, number 65-WA/HT-4, 1995.

[41] Martiny, M., Schulz, A., and Wittig, S. Mathematical model describing the coupled heat transfer in effusion cooled combustor walls. Proc. of ASME Turbo Expo, (97-GT-329), 1997.

[42] Kumada, M., Hirata, M., and Kasagi, N. Studies of full-coverage film cooling. II - Measurement of local heat transfer coefficient. ASME, Gas Turbine Conference and Products Show, 1981.

[43] Scrittore, J. J., Thole, K. A., and Burd, S. W. Investigations of velocity profiles for effusion cooling of a combustor liner. ASME J Turbomach, 129, July 2007.

[44] Schulz, A. Infrared thermography as applied to film cooling of gas turbine components. Meas. Sci. Technol., 11:948-956, 2000.

[45] Scrittore, J. and Thole, K. Experimental characterization of filmcooling effectiveness near combustor dilution holes. In Proceedings of ASME Turbo Expo, number GT2005-68704, 2005.

[46] Metzger, D.E., Takeuchi, D.I., and Kuenstler, P.A. Effectiveness and heat transfer with full-coverage film cooling. ASME J. Eng. Power, 95:180-184, 1973. 
[47] Martinez-Botas, R. F. and Yuen, C. H. N. Measurement of local heat transfer coefficient and film cooling effectiveness through discrete holes. Proc. of ASME Turbo Expo, (2000-GT-243), 2000.

[48] Facchini, B., Maiuolo, F., Tarchi, L., and Coutadin, D. Combined effect of slot injection, effusion array and dilution hole on the heat transfer coefficient of a real combustor liner - part 1 experimental analysis. Proc. of ASME Turbo Expo, (GT2010-22936), 2010.

[49] Andreini, A., Ceccherini, A., Facchini, B., and Coutandin, D. Combined effect of slot injection, effusion array and dilution hole on the heat transfer coefficient of a real combustor liner - part 2 numerical analysis. Proc. of ASME Turbo Expo, (GT2010-22937), 2010.

[50] Ceccherini, A. Design of Innovative Cooling Systems for AeroEngine Combustion Chambers. PhD thesis, University of Florence, Energy Engineering Department, 2009.

[51] Ligrani, P., Goodro, M., Fox, M., and Moon, H.-K. Full-coverage film cooling: Film effectiveness and heat transfer coefficients for dense and sparse hole arrays at different blowing ratios. ASME J. Turbomach., 134, 2012.

[52] Martin, D. and Thorpe, S. J. Experiments on combustor effusion cooling under conditions of very high free-stream turbulence. In Proceedings of ASME Turbo Expo, number GT2012-68863, 2012.

[53] Ekkad, S. V., Zapata, D., and Han, J. C. Heat transfer coefficient over a flat surface with air and $\mathrm{CO} 2$ injection through compound angle holes using a transient liquid crystal image method. ASME J. Turbomac., 119:580-585, 1997.

[54] Ekkad, S. V., Zapata, D., and Han, J. C. Film effectiveness over a flat surface with air and $\mathrm{CO} 2$ injection through compound angle holes using a transient liquid crystal image method. ASME J. Turbomac., 119:587-593, 1997. 
[55] Lin, Y., Song, B., Li, B., Liu, G., and Wu, Z. Investigation of film cooling effectiveness of full-coverage inclined multihole walls with different hole arrangements. Proc. of ASME Turbo Expo, 2003.

[56] Lin, Y., Song, B., Li, B., and Liu, G. Measured film cooling effectiveness of three multihole patterns. ASME Journal of Heat Transfer, 128:192-197, 2006.

[57] Facchini, B., Maiuolo, F., Tarchi, L., and Coutadin, D. Experimental investigation on the effects of a large recirculating area on the performance of an effusion cooled combustor liner. ASME J. Eng. Gas Turb. Power, 134, 2012.

[58] Wurm, B., Schulz, A., Bauer, H.J., and Gerendas, M. Impact of swirl flow on the cooling performance of an effusion cooled combustor liner. ASME J Gas Turb Pwr, (134):121503-1:121503-9, 2012.

[59] Wurm, B., Schulz, A., Bauer, H.J., and Gerendas, M. Cooling efficiency for assessing the cooling performance of an effusion cooled combustor liner. Proceedings of ASME Turbo Expo, (GT2013-94304), 2013.

[60] Andreini, A., Caciolli, G., Facchini, B., Picchi, A., and Turrini, F. Experimental investigation of the flow field and the heat transfer on a scaled cooled combustor liner with realistic swirling flow generated by a lean-burn injection system. ASME J Turbomach, (IN PRESS), 2014.

[61] Patil, S., Abraham, S., and Ekkad, S. Experimental and numerical investigation of convective heat transfer in a gas turbine can combustor. In Proceedings of ASME Turbo Expo, number GT2009-59377, 2009 .

[62] Wurm, B., Schulz, A., Bauer, H.-J., and Gerendas, M. Impact of swirl flow on the penetration behaviour and cooling performance of a starter cooling film in modern lean operating combustion chambers. Proceedings of ASME Turbo Expo, (GT2014-25520), 2014. 
[63] Strelets, M. Detached eddy simulation of massively separated flows. 39th Aerospace Sciences Meeting and Exhibit, (AIAA Paper 20010879), 2001.

[64] Menter, F. R. and Egorov, Y. Re-visiting the turbulent scale equation. Proc. IUTAM Symp. One Hundred Years of Boundary Layer Research, 2004.

[65] Menter, F. R. and Egorov, Y. A scale-adaptive simulation model using two-equation models. AIAA Paper-2005-1095, 2004.

[66] Widenhorn, A., Noll, B., and Aigner, M. Numerical study of a nonreacting turbulent flow in a gas turbine model combustor. $A I A A$ Paper 2009-64\%, 2009.

[67] Patil, S. and Tafti, D. Large eddy simulation of flow and convective heat transfer in a gas turbine can combustor with synthetic inlet turbulence. Proceedings of ASME Turbo Expo, (GT2011-46561), 2011.

[68] Crabb, D., Durao, D. F. G., and Whitelaw, J. H. A round jet normal to a crossflow. Trans. ASME: J. Fluids Eng, (103), 1981.

[69] Galeazzo, F. C. C., Donnert, G., Habisreuther, P., Zarzalis, N., Valdes, R. J., and Krebs, W. Measurement and simulation of turbulent mixing in a jet in crossflow. Proceedings of ASME Turbo Expo, (GT2010-22709), 2010.

[70] Schluter, J. U. and Schonfeld, T. LES of jets in cross flow and its application to a gas turbine burner. Flow, Turbulence and Combustion, 65(2):177-203, 2001.

[71] Mendez, S. and Nicoud, F. An adiabatic homogeneous model for the flow around a multi-perforated plate. AIAA Journal, 10(46): 2623-2633, 2008. 
[72] Hoda, A. and Acharya, S. Predictions of a film coolant jet in crossflow with different turbulence models. ASME J. Turbomach., 122:558-569, 2000.

[73] Harrison, K. L. and Bogard, D. G. Comparison of rans turbulence models for prediction of film cooling performance. Proceedings of ASME Turbo Expo, (GT2008-51423), 2008.

[74] Bacci, A. and Facchini, B. Turbulence modeling for the numerical simulation of film and effusion cooling flows. Proceedings of ASME Turbo Expo, (GT2007-27182), 2007.

[75] Holloway, D. S., Walters, D. K., and Leylek, J. H. Computational study of jet-in-crossflow and film cooling using a new unsteady-based turbulence model. Proceedings of ASME Turbo Expo, (GT2005$68155), 2005$.

[76] Bergeles, Gosman, G., and Launder, A. D. The turbulent jet in a cross stream at low injection rates: a three-dimensional numerical treatment. Numerical Heat Transfer, 1:217-242, 1978.

[77] Azzi, A. and Lakehal, D. Perspectives in modeling film cooling of turbine blades by transcending conventional two-equation turbulence models. ASME J. Turbomach., 124:472-484, 2002.

[78] Mangani, L. and Andreini, A. Application of an object oriented CFD code to heat transfer analysis. Proceedings of ASME Turbo Expo, (GT2008-51118), 2008.

[79] Andreini, A., Bianchini, C., Ceccherini, A., Facchini, B., Mangani, L., Cinque, G., and Colantuoni, S. Investigation of circular and shaped effusion cooling arrays for combustor liner application - Part 2: numerical analysis. Proceedings of ASME Turbo Expo, (GT200960038), 2009.

[80] Boust, B., Lalizel, G., Bianchini, C., Ceccherini, A., Cinque, G., and Colantuoni, S. Dual investigations on the improvement of effusion 
cooling by shaped holes. 7th World Conference on Experimental Heat Transfer, Fluid Mechanics and Thermodynamics, 2009.

[81] Andreini, A., Bianchini, C., Facchini, B., Mangani, L., and Maritano, M. Heat transfer performances of fan-shaped film cooling holes. Part II - numerical analysis. Proceedings of ASME Turbo Expo, (GT2010-22809), 2010.

[82] Cottin, G., Laroche, E., Savary, N., and Millan, P. Modeling of the heat flux for multi-hole cooling applications. Proceedings of ASME Turbo Expo, (GT2011-46330), 2011.

[83] Li, X., Ren, J., and Jiang, H. Algebraic anisotropic eddy-viscosity modeling application to the turbulent film cooling flows. Proceedings of ASME Turbo Expo, (GT2011-45791), 2011.

[84] L'Ecuyer, M. R. and Soechting, F. O. A model for correlating flat plate film-cooling effectiveness for rows of round holes. AGARDCP-390, 1985.

[85] Andreini, A., Facchini, B., Picchi, A., Tarchi, L., and Turrini, F. Experimental and theoretical investigation of thermal effectiveness in multi-perforated plates for combustor liner effusion cooling. ASME J. Turbomach., 136(9), 2014.

[86] Kaszeta, R. W. and Simon, T. W. Measurement of eddy diffusivity of momentum in film cooling flows with streamwise injection. ASME J. Turbomach., 122:178-183, 2000.

[87] Lakehal, D. Near-wall modeling of turbulent convective heat transport in film cooling of turbine blades with the aid of direct numerical simulation data. ASME J. Turbomach., 124:485-498, 2002.

[88] Kim, J., Moin, P., and Moser, R. Turbulence statistics in fully developed channel flow at low reynolds number. J. Fluid Mech., 177:133-166, 1987. 
[89] Bianchini, C., Andrei, L., Andreini, A., and Facchini, B. Numerical benchmark of non-conventional RANS turbulence models for film and effusion cooling. ASME J. Turbomach., 135(3), 2013.

[90] Andrei, L., Andreini, A., Bianchini, C., Facchini, B., and Mazzei, L. Numerical analysis of effusion plates for combustor liners cooling with varying density ratio. Proceedings of ASME Turbo Expo, (GT2013-95039), 2013.

[91] Andrei, L., Andreini, A., Bianchini, C., Caciolli, G., Facchini, B., Mazzei, L., Picchi, A., and Turrini, F. Effusion cooling plates for combustor liners: Experimental and numerical investigations on the effect of density ratio. Energy Procedia, 45(0):1402 - 1411, 2014.

[92] Kao, Y.-H., Tambe, S. B., and S.-M., Jeng. Aerodynamics study of a linearly-arranged 5-swirler array. Proceedings of ASME Turbo Expo, (GT2014-25094), 2014.

[93] Syred, N. Review of oscillation mechanisms and the role of the precessing vortex core (PVC) in swirl combustion systems. Elsevier Progress in Energy and Combustion Science, (32):93-161, 2006.

[94] Kline, S. J. and McClintock, F. A. Describing uncertainties in single sample experiments. Mechanical Engineering, 75(1):3-8, January 1953.

[95] Lefebvre, A. H. and Ballal, D. R. Gas Turbine Combustion. CRC Press - Taylor \& Francis Group, 2010.

[96] Menter, F. R. Best Practice: Scale-Resolving Simulations in ANSYS CFD. ANSYS Germany GmbH, April 2012.

[97] Spalart, P.R, Jou, W.-H., Strelets, M., and Allmaras, S.R. Comments on the feasibility of LES for wings, and on a hybrid RANS/LES approach. 1st AFOSR Int. Conf. On DNS/LES, 1997.

[98] Menter, F. R. and Kuntz, M. Development and application of a zonal DES turbulence model for CFX-5. ANSYS CFX, 2003. 
[99] Egorov, Y. and Menter, F. R. Development and application of sst-sas turbulence model in the desider project. Second Symposium on Hybrid RANS-LES Methods, 2007.

[100] Pope, S. B. Ten questions concerning the large-eddy simulation of turbulent flows. New Journal of Physics, 35, March 2004.

[101] Boudier, G., Gicquel, L. Y. M., and Poinsot, T. J. Effects of mesh resolution on large eddy simulation of reacting flows in complex geometry combustors. Elsevier Combustion and Flame, 155:196-214, October 2008.

[102] Andreini, A., Becchi, R., Facchini, B., Picchi, A., and Turrini, F. Effect of slot injection and effusion array on the liner heat transfer coefficient of a scaled lean burn combustor with realistic swirling flow. Proceedings of ASME Turbo Expo, (GT2015-42587), 2015.

[103] Andreini, A., Becchi, R., Facchini, B., Mazzei, L., Picchi, A., and Turrini, F. Adiabatic effectiveness and flow field measurements in a realistic effusion cooled lean burn combustor. Proceedings of ASME Turbo Expo, (GT2015-42584), 2015.

[104] Crawford, M. E., Kays, W. M., and Moffat, R. J. Full coverage film-cooling, part II: Heat trasfer data and numerical simulation. ASME J. Eng. Power, 102(4):1006-1012, 1980.

[105] Heidmann, J. D. and Hunter, S. D. Coarse grid modeling of turbine film cooling flows using volumetric source terms. Technical Report NASA TM-2001-210817, NASA, May 2001.

[106] Hunter, S. D. Source Term modeling of endwall cavity flow. PhD thesis, Division of Reasearch and Advanced Studies of the University of Cincinnati, 1998.

[107] Tartinville, B. and Hirsch, C. Modelling of film cooling for turbine blade design. Proceedings of ASME Turbo Expo, GT2008-50316, 2008. 
[108] Burdet, A., Abhari, R. S., and Rose, M. G. Modeling of film cooling - part II: model for use in three-dimensional computational fluid dynamycs. ASME J Turbomach, 129, April 2007.

[109] auf dem Kampe, T. and Völker, Stefan. A model for cylindrical hole film cooling: part II: model formulation, implementation and results. ASME J Turbomach, 134, September 2012.

[110] Mendez, S. and Nicoud, F. Adiabatic homogeneous model for flow around a multiperforated plate. AIAA Journal, 46(10):2623-2633, 2008 .

[111] Rida, S., Reynolds, R., Chakracorty, S., and Gupta, K. Imprinted effusion modeling and dynamic $C_{D}$ calculation in gas turbine combustors. Proceedings of ASME Turbo Expo, GT2012-68804, 2012.

[112] Voigt, S., Noll, B., and Aigner, M. Development of a macroscopic CFD model for effusion cooling applications. Proceedings of ASME Turbo Expo, GT2012-68251, 2012.

[113] ANSYS CFX-Solver Modeling Guide, release 14.0 edition, November 2011 .

[114] Cho, H. H. and Goldstein, R. J. Heat (mass) transfer and film cooling effectiveness with injection through discrete hole: Part I within holes and on the back surface. ASME J Turbomach, 117: 440-450, 1995.

[115] Andreini, A., Bonini, A., Caciolli, G., Facchini, B., and Taddei, S. Numerical study of aerodynamic losses of effusion cooling holes in aero-engine combustor liners. ASME J Gas Turb Pwr, 133(2), 2011.

[116] Andreini, A., Facchini, B., Picchi, A., Tarchi, L., and Turrini, F. Experimental and theoretical investigation of thermal effectiveness in multi-perforated plates for combustor liner effusion cooling. Proceedings of ASME Turbo Expo, (GT2013-94667), 2013. 
[117] Andrei, L., Andreini, A., Bianchini, C., Facchini, B., and Mazzei, L. Numerical analysis of multi perforated effusion cooling plates for combustor liners with varying density ratio. Proceedings of ASME Turbo Expo, GT2013-95039, 2013.

[118] Andreini, A., Ceccherini, A., Facchini, B., Turrini, F., and Vitale, I. Assessment of a set of numerical tools for the design of aero-engines combustors: study of a tubular test rig. Proceedings of ASME Turbo Expo, GT2009-59539, 2009.

[119] Andreini, A., Caciolli, G., Facchini, B., Picchi, A., and Turrini, F. Experimental investigation of the flow field and the heat transfer on a scaled cooled combustor liner with realistic swirling flow generated by a lean-burn injection system. In Proceedings of ASME Turbo Expo 2014, number GT2014-25643, 2014.

[120] Ivanova, E. M. A numerical study on the turbulent Schmidt numbers in a jet in crossflow. ASME J Eng Gas Turb Power, 135, January 2013.

[121] Jiang, L.-Y. and Campbell, I. Prandtl/Schmidt number effect on temperature distribution in a generic combustor. International Journal of Thermal Sciences, 48:322-330, April 2008.

[122] De Champlain, A., Gosselin, P., and Kretschmer, D. Prediction of wall heat transfer for a gas turbine combustor. Proc. Instn. Mech. Engrs., 213:169-180, 1999.

[123] Facchini, B., Surace, M., and Zecchi, S. A new concept of impingement cooling for gas turbine hot parts and its influence on plant performance. Proc. of ASME Turbo Expo, (GT2003-38166), 2003.

[124] Facchini, B., Surace, M., and Tarchi, L. Impingement cooling for modern combustors: experimental analysis and preliminary design. Proc. of ASME Turbo Expo, GT2005-68361, 2005. 
[125] Arcangeli, L., Facchini, B., Surace, M., and Tarchi, L. Correlative analysis of effusion cooling systems. ASME J. Turbomach., 130 (011016), 2008.

[126] Andreini, A., Carcasci, C., Ceccherini, A., Facchini, B., Surace, M., Coutandin, D., Gori, S., and Peschiulli, A. Combustor liner temperature prediction: a preliminary tool development and its application on effusion cooling systems. First CEAS European Air and Space Conference Century Perspectives, (026), 2007.

[127] Andreini, A., Ceccherini, A., Facchini, B., Turrini, F., and Vitale, I. Assesment of a set of numerical tools for the design of aeroengines combustors: study of a tubular test rig. Proc. of ASME Turbo Expo, GT2009-59539, 2009.

[128] Farag, I. H. Non luminous gas radiation - approximate emissivity models. Heat Trasfer 1982 - Proceedingsof The Seventh International Heat Transfer Conference, 1982.

[129] Gnielinsky, V. New equations for heat and mass transfer in turbulent pipe channel flow. Int. Chem. Eng., 16:359, 1976.

[130] L'Ecuyer, M. R. and Soechting, F. O. A model for correlating flat plate film-cooling effectiveness for rows of round holes. AGARDCP-390, 1985.

[131] Baldauf, S., Scheurlen, M., Schulz, A., and Wittig, S. Correlation of film-cooling effectiveness from thermographic measurements at enginelike conditions. ASME J. Turbomach., 124(4):686-698, 2002.

[132] Sellers Jr., J. P. Gaseous film cooling with multiple injection stations. AIAA J., 1(9):2154-2156, 1963.

[133] Andreini, A., Carcasci, C., Gori, S., and Surace, M. Film cooling system numerical design: Adiabatic and conjugate analysis. In Proceedings of HT2005, number HT2005-72042, 2005. 
[134] Da Soghe, R., Andreini, A., Facchini, B., and Mazzei, L. Heat transfer enhancement due to coolant extraction on the cold side of effusion cooling plates. In Proc. of ASME Turbo Expo, number GT2014-25393, 2014.

[135] Da Soghe, R. and Andreini, A. Numerical characterization of pressure drop across the manifold of turbine casing cooling system. ASME J. Turbomach., 135(031017), May 2013.

[136] Incropera, F. P., DeWitt, D. P., Bergman, T. L., and Lavine, A. S. Fundamentals of Heat and Mass Transfer. John Wiley \& Sons, sixth edition, 2006.

[137] Balthasar, M., Mauss, F., Pfitzner, M., and Mack, A. Implementation and validation of a new soot model and application to aeroengine combustors. ASME J Gas Turb Pwr, 124(66-74), January 2002. 